\title{
Handbook of best practice and standards for 2D+ and 3D imaging of natural history collections
}

\author{
Jonathan BRECKO ${ }^{1, *}$ \& Aurore MATHYS ${ }^{2}$ \\ ${ }^{1,2}$ Royal Belgian Institute of Natural Sciences, Scientific Heritage Service, \\ Vautierstraat 29, B-1000 Brussels, Belgium. \\ ${ }^{1,2}$ Royal Museum for Central Africa, Biological Collections and Data Management, \\ Leuvensesteenweg 13, B-3080 Tervuren, Belgium. \\ *Corresponding author: jbrecko@naturalsciences.be \\ 2Email: amathys@naturalsciences.be \\ ${ }^{1}$ urn:1sid:zoobank.org:author:7AC9797B-88EB-4844-86B9-C88DF7C06B2E \\ ${ }^{2}$ urn:lsid:zoobank.org:author:0C719566-2901-471D-B88E-CE3EBB476172
}

With contributions* from Bernhard Ströbel, Michael Heethoff, Susan Stephan, Bernhard Schurian, Didier VandenSpiegel and Patrick Semal.

\begin{abstract}
Digitising a collection is key to make it last even after the physical objects are no longer available. Almost all of the techniques currently available to digitise a natural history collection in 2D+ and $3 \mathrm{D}$ are listed herein. The techniques are explained in a way that even one without any knowledge on the subject may understand their principle. The strong and weak points of the techniques are discussed, and an overview of suitable collections and specimens are given for each one of them. Also, plenty of examples already digitised with each technique are provided together with the links to visualise them in 3D. After explaining all the different digitisation options, the subsequent chapters provide information on how to improve the 2D+ and 3D digital twins of the specimens and techniques are compared to each other by means of test specimens. These give a fast overview of the capabilities of the digitisation techniques. Possible solutions to avoid digitisation errors are equally provided. Lastly, the dissemination of the results and the data management of the 3D models are briefly discussed in the final chapters. Also, a large chapter is provided with several workflows that can be followed to get the best possible results.
\end{abstract}

Keywords. Digitisation, handbook, 3D, natural history collections, 2D+.

Brecko J. \& Mathys A. 2020. Handbook of best practice and standards for 2D+ and 3D imaging of natural history collections. European Journal of Taxonomy 623: 1-115. https://doi.org/10.5852/ejt.2020.623

*Affiliations and details regarding the contributions can be found on the page 114. 


\section{Table of contents}

Foreword .

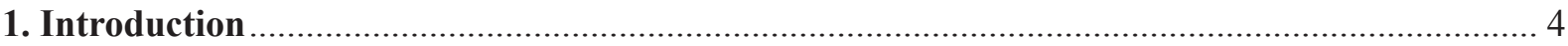

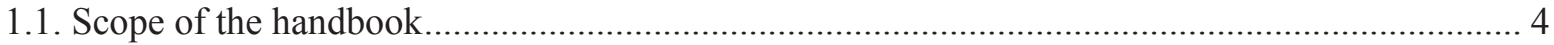

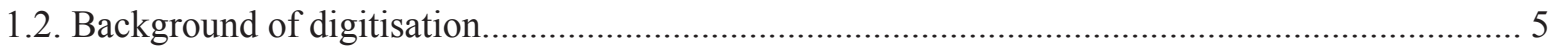

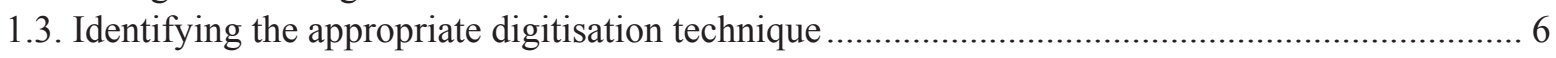

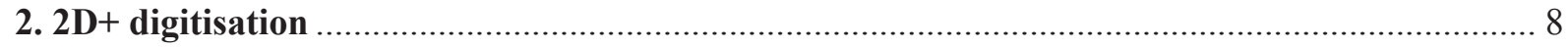

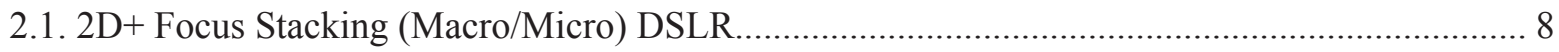

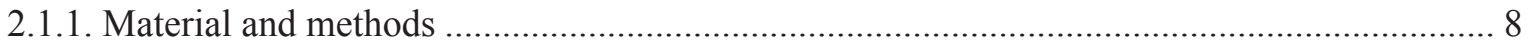

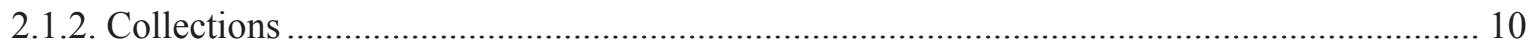

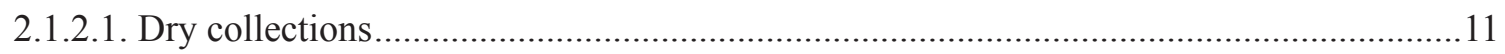

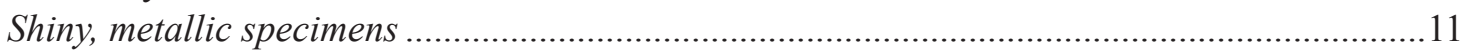

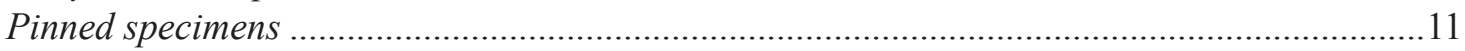

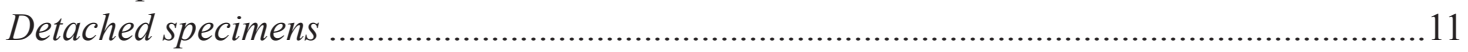

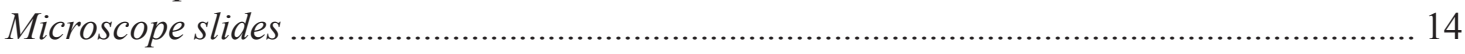

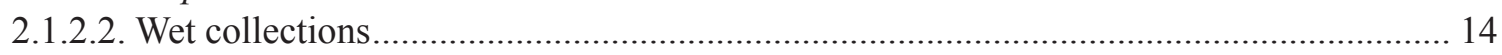

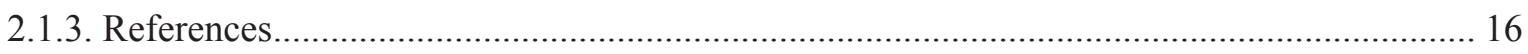

2.2. 2D+ Focus Stacking (Macro/Micro) Microscope .................................................................. 18

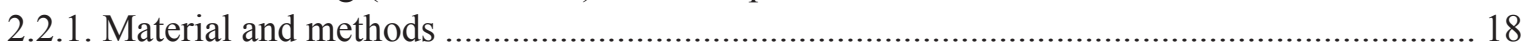

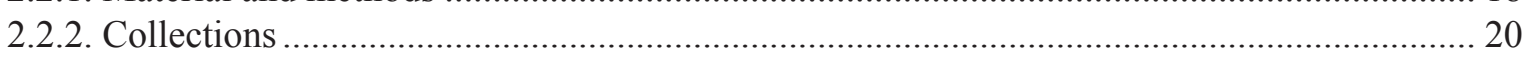

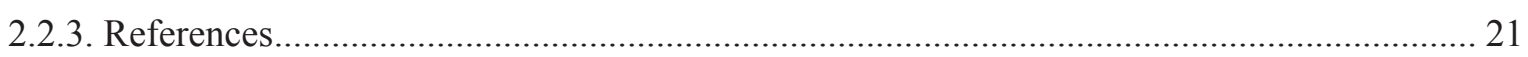

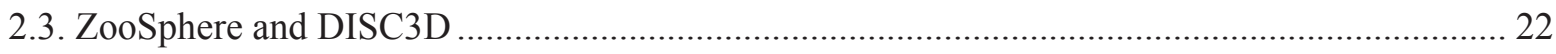

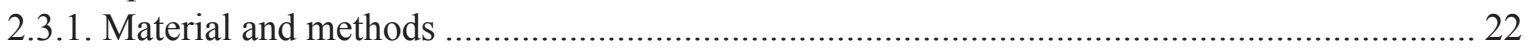

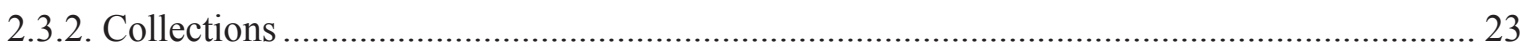

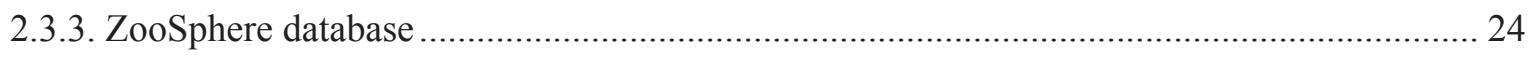

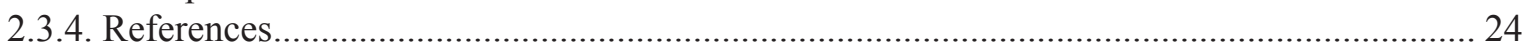

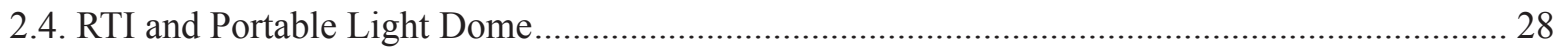

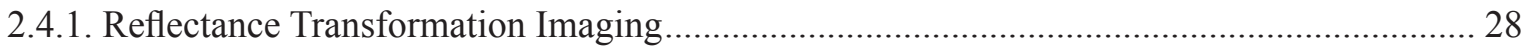

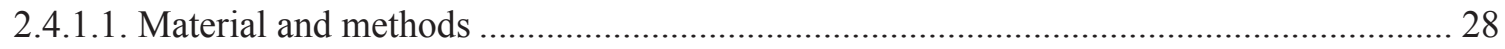

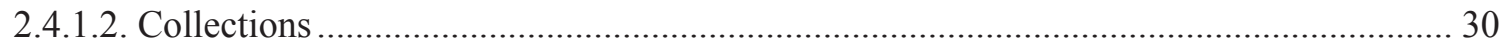

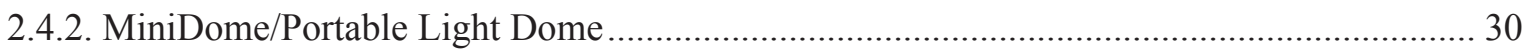

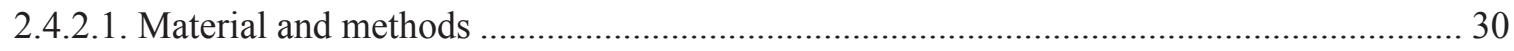

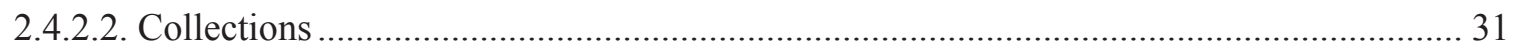

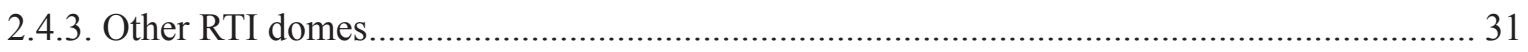

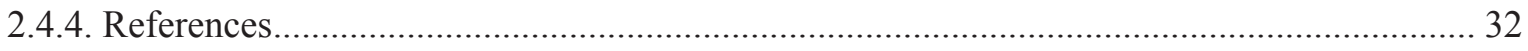

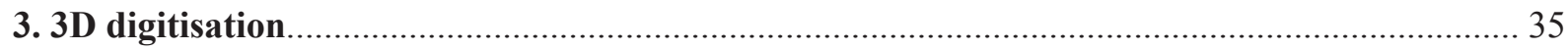

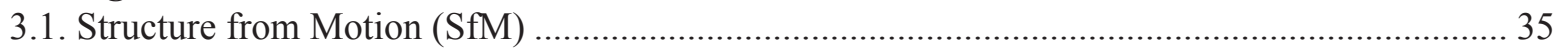

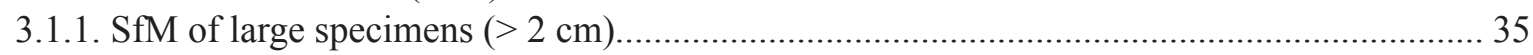

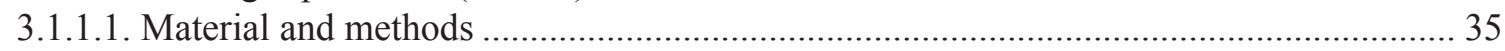

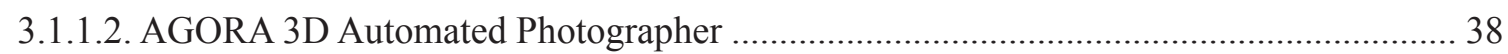

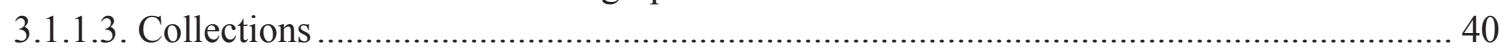

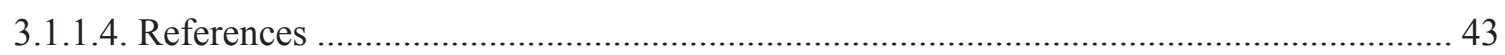

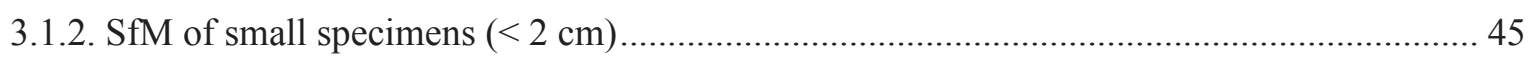

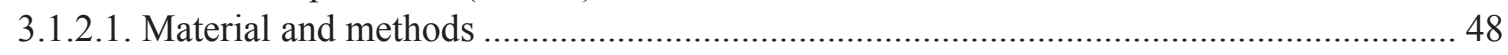

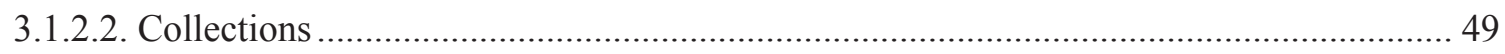

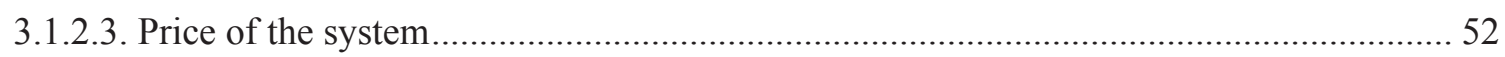

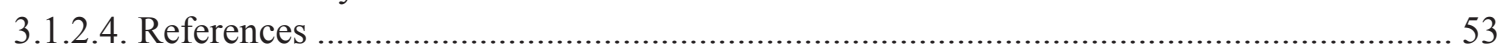

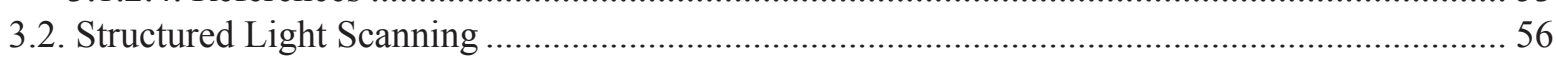

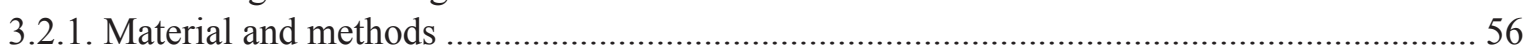


BRECKO J. \& MATHYS A., Best practice handbook for 2D+/3D digitisation

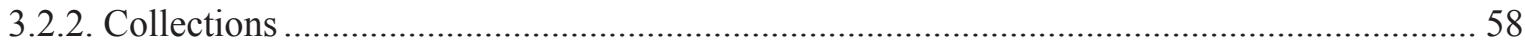

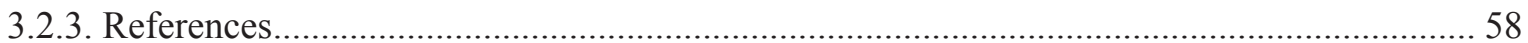

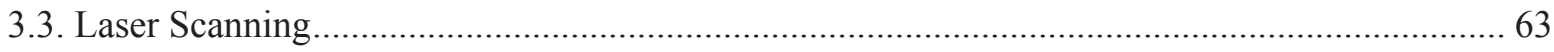

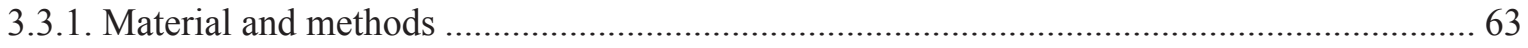

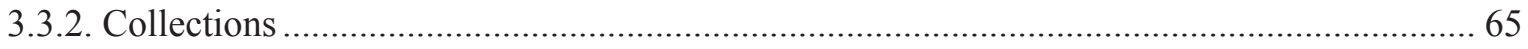

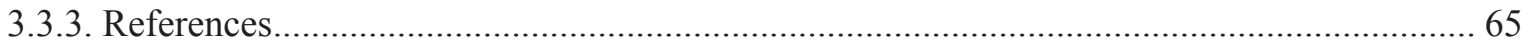

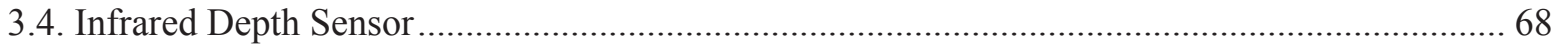

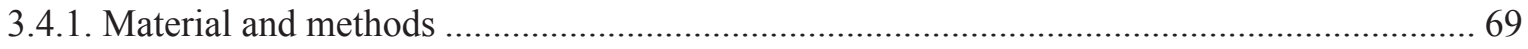

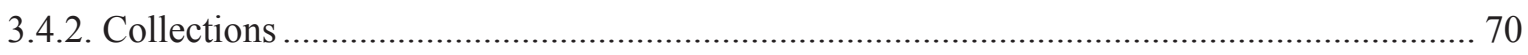

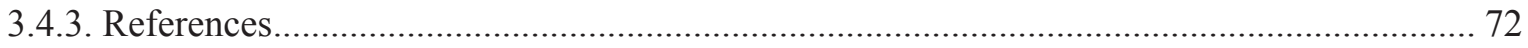

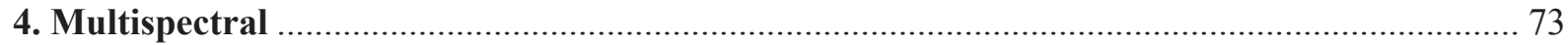

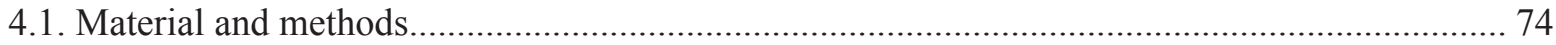

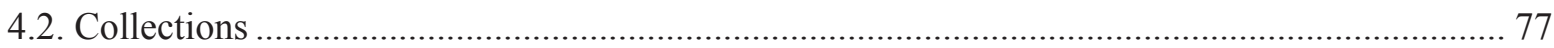

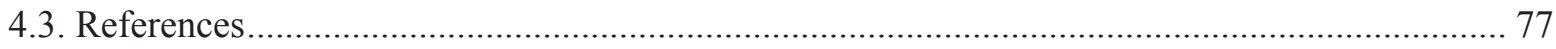

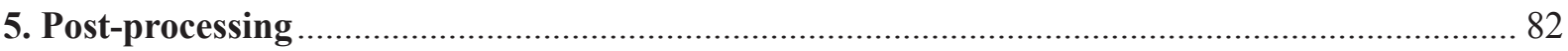

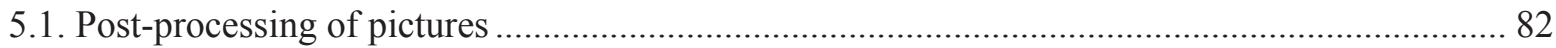

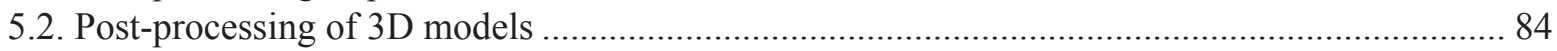

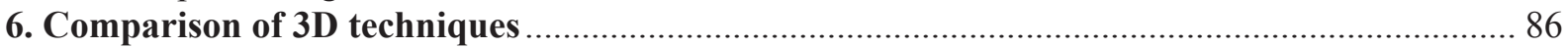

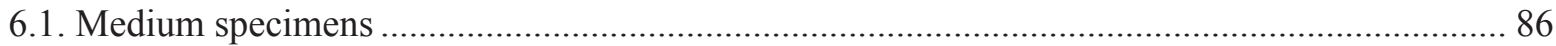

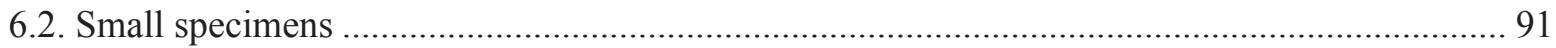

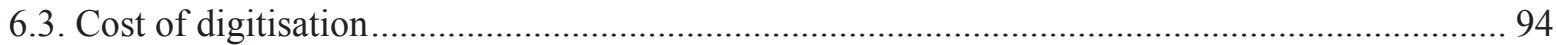

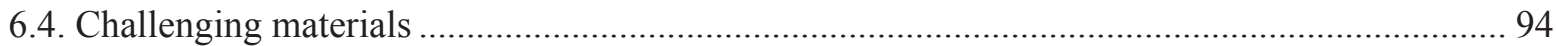

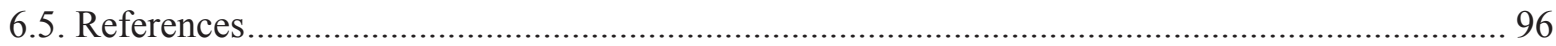

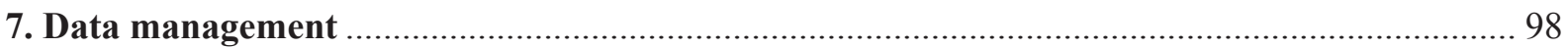

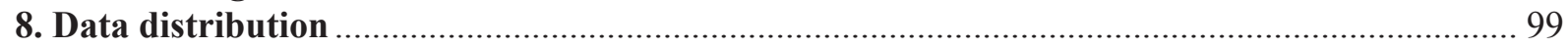

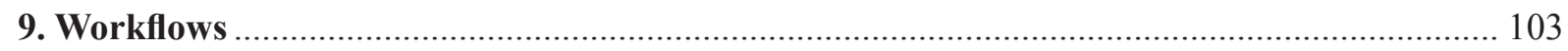

9.1. Workflow 2D+ Focus Stacking DSLR @ RBINS and RMCA.................................................. 103

9.2. Workflow Microscope (Information in Imaging) @ RBINS and RMCA................................... 105

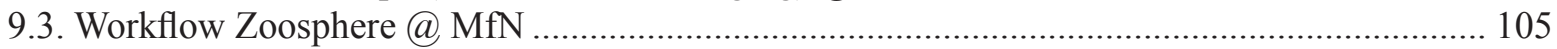

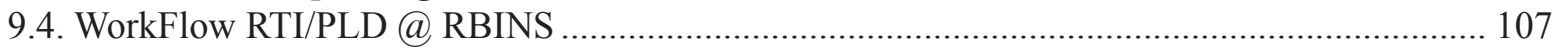

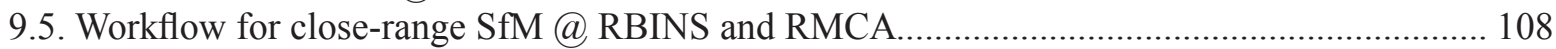

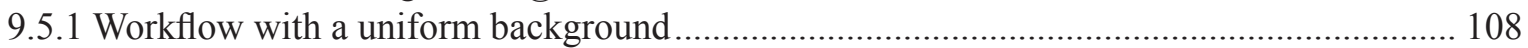

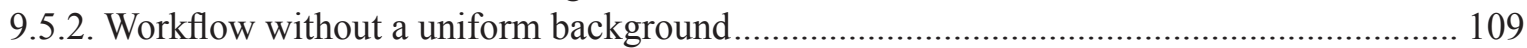

9.6. Workflow Structured Light Scanner (HDI and MechScan) @ RBINS.......................................110

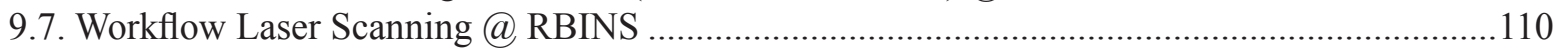

9.8. Workflow Infrared Depth Sensors @ RMCA and RBINS .........................................................111

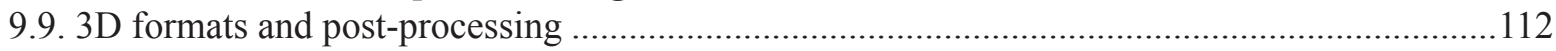

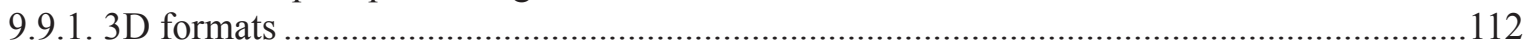

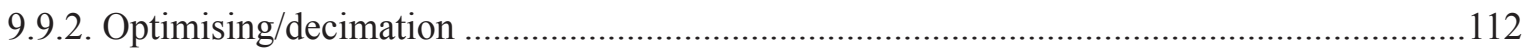

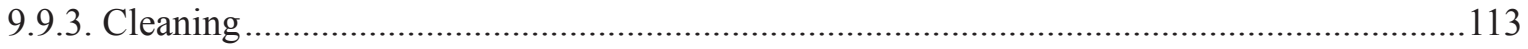

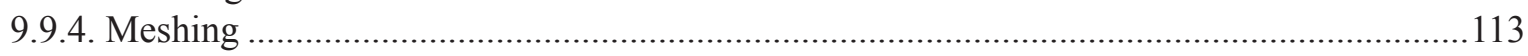

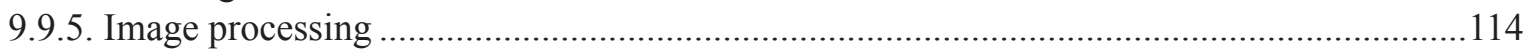

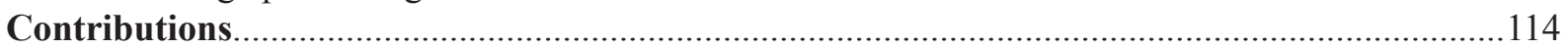




\section{Foreword}

\section{SYNTHESYS 3: Network Activity 2}

This handbook is created under Objective 1, Task 1.2 of Network Activity 2 in the SYNTHESYS 3 project. SYNTHESYS is an EC-funded project creating an integrated European infrastructure for natural history collections. SYNTHESYS 3 started in 2013 and ended in 2017 (http://www.synthesys.info/)

From www.synthesys.info: "SYNTHESYS is continuing its work into improving access to and management of Natural History Collections, with a particular focus on the issues relating to modern technology and the digital data this produces. The SYNTHESYS 3 Network Activities (NA) will provide a coherent, integrated and sustainable management approach to virtual and new physical collections that will enable institutions to meet the demands of both current and future users. Outputs will include collection management policies, protocols and best practice guides."

NA 2: Improving collections management and enhancing accessibility. The tools, standards and training provided through the NAs of SYNTHESYS 1 and 2 have proved successful in improving collections management of traditional European natural history collections (e.g., alcohol-preserved specimens, desiccated plants and insects, bones and minerals). There is now a demand from both natural history institutions and the user community to move the focus from improving collections management of traditional collections to developing their growing virtual and new physical collections.

NA 2 will meet the demand by developing collection management policies and strategic priorities for open and flexible access to virtual and new physical collections.

\section{Objective 1: Managing new (virtual and physical) collections; Task 1.2: Produce a handbook of best practice and standards for 3D imaging of natural history specimens}

To get an overview of relevant 3D techniques, software and equipment, a handbook of best practice for 3D imaging will be produced. The handbook will use Joint Research Activities (JRA) Obj. 2 results and include recommendations concerning which techniques should be used for different types of objects, and which technical standards exist for creating a 3D image and for visualisation of the images.

International groups working on 3D imaging will be invited to a workshop to discuss the handbook content and to horizon-scan the domain. Chapters will be written by working groups constituted at the workshop (including others deemed relevant). The results will be used by JRA Obj. 4 .

All of the outcomes and reports from the SYNTHESYS 3 project can be found on the platform http://synthesys3.myspecies.info/.

\section{Introduction}

\subsection{Scope of the handbook}

The scope of this handbook is to present the different digitisation techniques currently in use in European natural history institutions. The aim is to provide a guideline for starting digitisation initiatives by showing which techniques can be used to digitise a certain collection or specimen. In this handbook of best practice, one will find, besides the general information of a certain technique, a thorough workflow, an equipment list and test cases on how to create a good digital replica of a specimen. Of course, workflows will evolve as software changes and hardware improves. To keep workflows up to date, a 3D handbook WIKI is produced and hosted by the Museum für Naturkunde Berlin (2 Jul. 2018, http://biowikifarm.net/v-mfn/3d-handbook/3d_Imaging_Handbook:Main_Page; see the contents section for workflows per technique). 
Although the handbook is mainly dedicated to best practice in 3D digitisation techniques, 2D digitisation is briefly discussed too, as this type of digitisation has been widely used to digitise 2D specimens (archives, herbarium sheets, microscope slides, etc.) as well as numerous 3D specimens. Many previous digitisation initiatives have focused on 2D digitisation. For this reason, many institutes have already have invested in these 2D techniques and have staff that are trained to use them, making it pertinent to cover them in the current handbook. Another reason for dedicating a chapter to $2 \mathrm{D}$ digitisation techniques is that many of these techniques are the basis for 3D digitisation, or can be combined with 3D digitisation to achieve better results. These 2D techniques will be discussed briefly, although the 2D+ (Extended Depth of Field, i.e., focus stacking) technique will be more thoroughly discussed.

At the Royal Belgian Institute of Natural Sciences (RBINS) and the Royal Museum for Central Africa (RMCA), daily 3D digitisation efforts began in 2014 with the start of the DIGIT03 project. This project was launched after a 2-year period of thoroughly testing almost all of the 3D techniques available at that time within the AGORA3D project funded by the Belgian Science Policy (BELSPO). The basis for this handbook was therefore developed within the AGORA3D project and the subsequent years of digitisation during the DIGIT03 and 3Dspectral projects.

\subsection{Background of digitisation}

To preserve, to study, and to present collections are three important principles of any museum. Technological developments, as well as evolution in public interest, have gradually transformed possibilities for natural history institutions and the various challenges they are facing.

Because of technological developments, there are more requests for specimen analyses that often require destructive sampling. This evolution of practice also means that new material often does not make it into the physical collections of scientific institutions. Therefore a "backup" of the collection specimen before its destruction is needed. Also, the collections and the way of collecting objects have completely changed over the last 50 years. It is not always possible to export objects from their country of origin to study them. Although a digital copy does not fully replace the real object, digitising them on-site could provide research opportunities in the absence of the original specimen. Digitising existing collections can also provide extra possibilities or alternatives for researchers to continue their work without removing specimens from their well-conditioned, secure and optimal storage facility. Or in the case that the country of origin cannot provide the conditions needed to securely store and maintain a collection, access to digital copies of the specimens could act as a substitute. One also has to consider that precious specimens can be destroyed in human and natural catastrophes and thus need to be preserved in any possible way. Finally, the expectations of the public have increased and there is a large demand for more information through the bias of new and innovative media.

High resolution digitisation can solve these problems, allowing:

- the creation of a digital twin with the highest possible resolution (at the time of digitisation) in order to preserve as much of the original data of the specimen as possible and make the digital twin usable for as much research as possible;

- the increase in value of collections in terms of quantity and scientific rigor;

- access to repatriated specimens for scientific studies;

- the creation of physical reproductions for display in museums.

It is important to note that the 3D digital twin that is created by the process of digitisation allows for research to take place that was not previously possible. The digital twin of any object or specimen now makes it easy to describe its spatial structure and opens the door for 3D geometric morphometrics, calculation of surface areas, estimation of volume, aid in ecomorphology studies, aid in new industrial 
developments and, in the event of $\mu \mathrm{CT}$ scans (discussed in the other volume on best practices, Keklikoglou et al. 2019), look at inner structures, calculate local thickness of the external surface, etc. In this regard, digitisation allows researchers to retrieve more information than was ever possible before from studying the original specimen. But as new techniques develop it remains important to preserve the original object or specimen to allow further research to be carried out on it using future techniques.

\subsection{Identifying the appropriate digitisation technique}

If it is not very clear which digitisation technique would be the most appropriate for a given collection, the following short key should help to determine this and find the corresponding chapter:

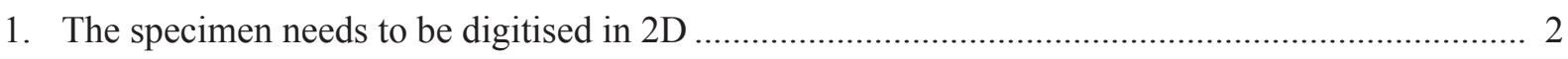

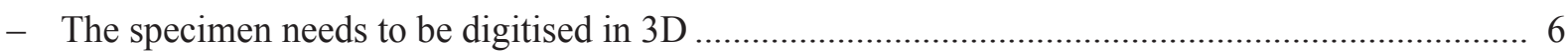

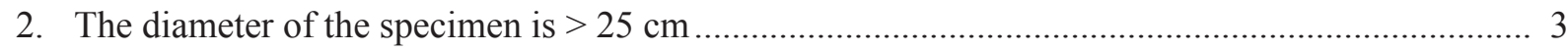

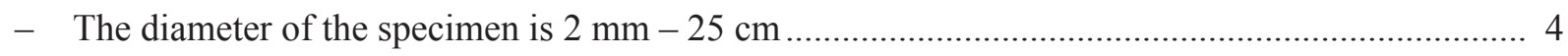

- The diameter of the specimen is $<2 \mathrm{~mm}$... 2.2. 2D+ Focus Stacking (Macro/Micro) Microscope

3. The specimen can be pictured using simple studio photography, which is not discussed in this handbook. Use a photo studio with soft boxes to create a smooth light or place the specimens in a light tent when picturing them. Some tips on basic photography can be found in the chapter 3.1 as this is also important for Structure from Motion.

4. The features, structures or key morphologies of the specimens are only visible when light comes from a certain direction or when light is moved across the specimen, or are not very clear in indirect, smooth light conditions like in a light tent

2.4. RTI and Portable Light Dome

- The features, structures or key morphologies of the specimens are clearly visible in indirect light conditions, but the depth of field is not sufficient to capture all the details

5. The complete specimen is important, but the necessary information can be captured by picturing a few manually selected views

2.1. 2D+ Focus Stacking (Macro/Micro) DSLR

- The complete specimen is important, therefore, it is necessary that all the views possible are pictured

2.3. Zoosphere and DISC3D

6. The 3D digital twin of the specimen must show the colour information (texture) of the specimen 7

- For the 3D digital twin of the specimen, the texture of the specimen is less important than the detail of the mesh (the 3D model) that will be created.

7. The diameter of the specimen is $>2 \mathrm{~cm}$

3.1.1. SfM of large specimens

- The diameter of the specimen is $<2 \mathrm{~cm}$, but larger than $2 \mathrm{~mm}$

3.1.2. SfM of small specimens

- The diameter of the specimen is $<2 \mathrm{~mm}$

Unfortunately, it is not possible to digitise the specimen with a colour texture. The best option is to use a $\mu \mathrm{CT}$ scanner (protocols available in the $\mu \mathrm{CT}$ handbook by Keklikoglou et al. 2019) and map a texture obtained by the technique in Chapter 2.2. 2D+ Focus Stacking (Macro/Micro) Microscope. 


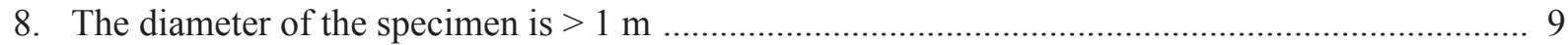

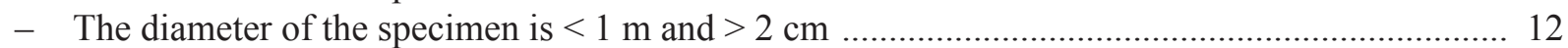

- The diameter of the specimen is $<2 \mathrm{~cm}$ and $>2 \mathrm{~mm}$..................... 3.1.2. SfM of small specimens

- The diameter of the specimen is $<2 \mathrm{~mm}$

$\boldsymbol{\mu C T}$ scanner (protocols available in Keklikoglou et al. 2019)

9. The specimen is easy to move and to handle, so a lot of space is available around it to scan ...... 10

- The specimen is very heavy, difficult to move, or the space around it is limited ........................... 11

10. The specimen has small details which are only visible with a good and detailed mesh and it is certain that the texture is less important

3.2. Structured Light Scanning

- The specimen has small details which are only visible with a good and detailed mesh but at this stage of the project it is not clear if the texture information can be ignored

3.1.1. SfM of large specimens

- The specimen has no particular detailed information that needs to be visible on the 3D model; the general shape of the specimen is enough

3.4. Infrared Depth Sensor

11. The specimen has small details which are only visible with a good and detailed mesh

3.1.1. SfM of large specimens

- The specimen has no particular detailed information that needs to be visible on the 3D model; the general shape of the specimen is enough

3.4. Infrared Depth Sensor

12. The specimen has small details which are only visible with a good and detailed mesh and it is certain that the texture is less important. The specimen can have a reflective, shiny surface and cannot be sprayed with coating to create a matt surface 3.2. Structured Light Scanning

- The specimen has small details which are only visible with a good and detailed mesh and it is certain that the texture is less important. The specimen is matt, non-reflective or can be sprayed with a coating ...... 3.3. Laser Scanning

- The specimen has small details which are only visible with a good and detailed mesh but at this stage of the project it is not clear if the texture information can be ignored 


\section{2D+ digitisation}

\subsection{D+ Focus Stacking (Macro/Micro) DSLR}

Museum collections harbour a huge diversity of specimens, all in different shapes, sizes and colours. Today, many techniques exist to digitise both natural history and cultural heritage collections. Several of these are already successfully tested and compared with each other (Jecic \& Dvar 2003; Hess \& Robson 2012; Hess et al. 2014; Mallison et al. 2009; Mallison et al. 2011; Mathys et al. 2013a, 2013b, 2014, 2015). However, the digitisation solution for small $(<2 \mathrm{~cm})$ specimens of a collection is not always straightforward, as many techniques fail to reconstruct a 3D model of these. Mostly the only relevant methods are $\mu \mathrm{CT}$ (micro Computed Tomography) and SEM (Scanning Electron Microscopy) imaging, which are unable to record colour information and have resolution restrictions imposed by equipment properties and sample size. Additionally, to obtain a 3D model from a $\mu \mathrm{CT}$ scan, which generates volumes consisting of voxels, it is necessary to perform a segmentation so a mesh can be computed based on the selection of the voxels within a region of interest. This can be a very time-consuming process and fine detail is easily lost if the segmentation process is not done carefully. Furthermore, $\mu \mathrm{CT}$ is not only quite expensive; the data gathered are also fairly large, demanding powerful computers, special software and large storage capacities with specific infrastructure for online sharing. The latter can be a problem for researchers that do not have access to good internet connection. Sometimes the inside of the object is not required, or even a complete 3D is not entirely necessary. SEM imaging can provide a solution but it can also be time consuming as the specimen often needs to be dried and gold-coated, which is not always desirable for type specimens. The best and fastest solution for the digitisation of such small objects might be taking $2 \mathrm{D}+$ pictures by means of focus stacking (Brecko et al. 2014).

\subsubsection{Material and methods}

For the focus stacking purposes the setup described by Brecko et al. (2014) is used, which consists of

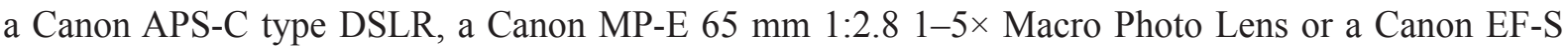
$60 \mathrm{~mm} \mathrm{f} / 2.8$ Macro USM, 2 flashes (Yongnuo YN560-IV, Neewer TT560, or similar), one remote control for the flash, a Cognisys StackShot and an Ikea "Metod" kitchen closet (Table 2.1; Fig. 2.1). A Canon

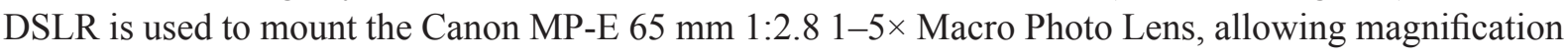
up to $5 \times$ (using an APS-C sensor eq. $8 \times$ on a full frame sensor). In case the camera equipment of one's photography lab is not from Canon, but one wishes to make a similar setup, an almost similar lens exists, the Laowa $25 \mathrm{~mm} \mathrm{f} / 2.82 .5-5 \times$ Ultra Macro, possible to mount on other brands of DSLRs (Venus Lens 2018).

Several background colours in different materials are used depending on the specimen. To get a smooth background it is necessary to create a distance between the background and the photographed object. This can be done by placing the specimen on an upside-down acrylic or glass petri dish if the object is not pinned or glued on a label like most insects. It is important though to make sure the surface of the petri dish is clean and free from scratches as this prevents the need of editing the picture afterwards. In case the specimen is pinned, it can be pinned directly to the background or placed in a specimen manipulator (Dupont et al. 2015) with a background underneath it. In this way a smooth background is created without any unwanted parts or objects visible.

Canon EOS Utility software is used to control the camera. Zerene Stacker is used for stacking the individual pictures into one 'stacked image' as described in Brecko et al. 2014.

Once the pictures are processed a scale bar is added, which is calculated according to following formula: $(\mathrm{A} / \mathrm{B}) \times \mathrm{C}$, where $\mathrm{A}=$ magnification, $\mathrm{B}=$ sensor size $(\mathrm{mm})$ and $\mathrm{C}=$ image width (pixels). 
Table 2.1. Equipment used for the 2D+ DSLR Setup at RBINS and RMCA

\begin{tabular}{c}
\hline Equipment \\
\hline Zerene Stacker Software \\
Canon DSLR (APS-C type) \\
Canon $65 \mathrm{~mm}$ MP-E f/2.8 1-5× Super Macro \\
Canon $60 \mathrm{~mm} \mathrm{f/2.8} \mathrm{EF} \mathrm{Macro}$ \\
Cognisys StackShot \\
Shutter Speed Cable \\
$2 \times$ Speedlights (Yongnuo or Neewer) \\
Remote control for Speedlight
\end{tabular}

In this way the number of pixels for $1 \mathrm{~mm}$ is calculated. For a magnification of $5 \times$ using a Canon APS-C DSLR (sensor size $22.3 \mathrm{~mm}$ ) and an image width of 5184 pixels, $1 \mathrm{~mm}$ is represented in the picture by 1162.3 pixels, or otherwise stated 1 pixel corresponds to $0.86 \mu \mathrm{m}$.

Although in this way the pictures are 'scaled' it is clear that these scales can not be used to measure parts visible on the picture. The way a focus stacked image is formed implies that the specimen will have different scales during the picturing process. When the lens is far from the specimen, and the focal plane is on the first structures of the specimen, the specimen will be smaller on the image than when the part which is in focus is the farthest away and hence the lens is closer to the specimen. So there is not only a shift in the focal plane, but also in the scaling during the picturing process. A quantitative data analysis of the specimen on $2 \mathrm{D}+$ images is therefore not possible and this needs to be considered if the output needs to be used for such a research purpose, like geometric morphometrics for instance.

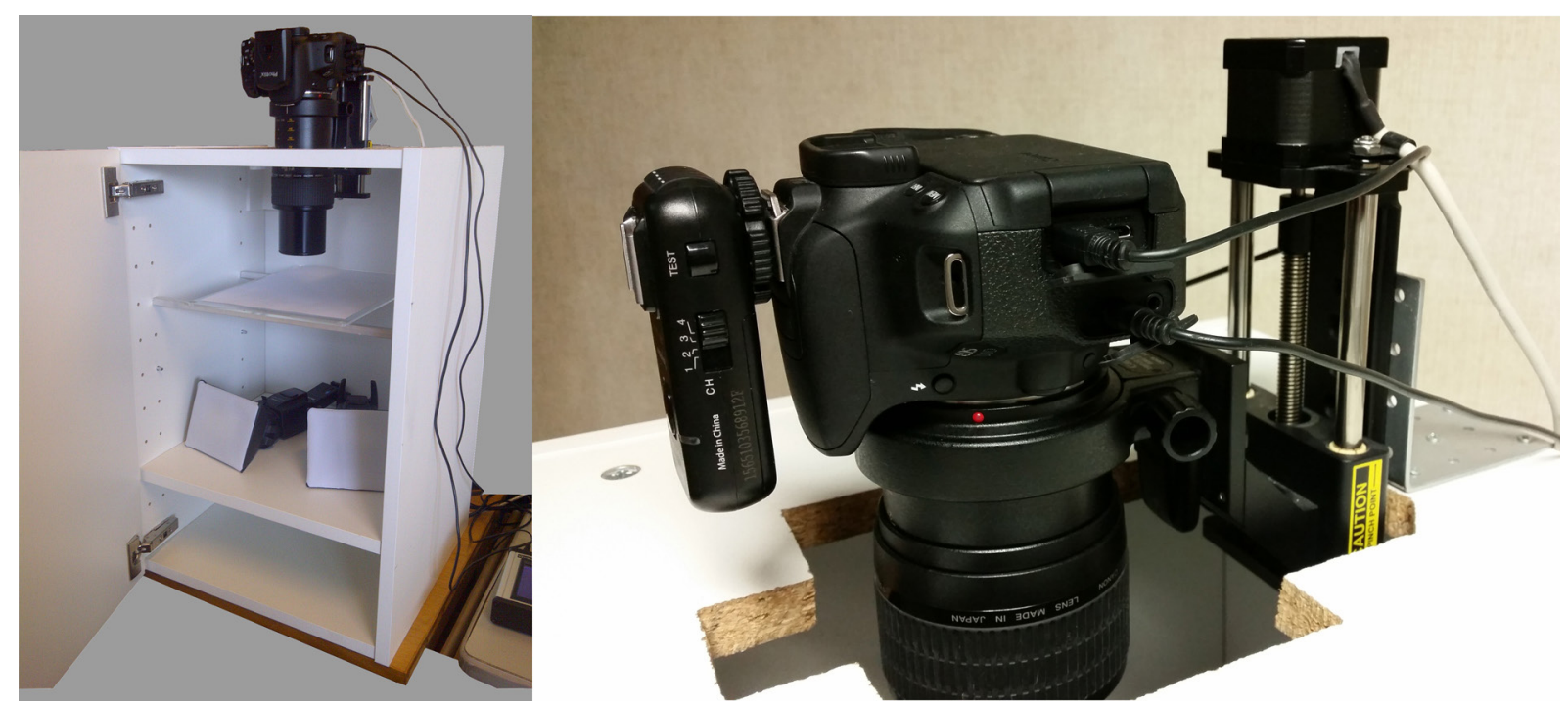

Fig. 2.1. Focus Stacking setup used at the Royal Belgian Institute of Natural Sciences (RBINS) and the Royal Museum for Central Africa (RMCA). Left: View of the complete set-up; Right: Detailed view of the camera mounted to stackshot and to the closet using a metal corner, to mount the camera vertically. 
The Canon APS-C type DSLR is used because tests and calculations show that at 50.6 megapixel on a $36 \mathrm{~mm}$ sensor (full frame), the amount of $\mu \mathrm{m}$ per pixel is almost equal when using the 'same magnification' of the lens on a $22.3 \mathrm{~mm}$ sensor (Table 2.2). The quality of the pictures is nearly identical, maybe slightly higher, when using the Canon EOS 5DS R (Fig. 2.2). However, in our opinion, it is not enough to justify the difference in price for this application.

Table 2.2. Equipment list needed to perform photogrammetry of small specimens.

\begin{tabular}{ccc|ccccc}
\hline Camera & & \multicolumn{5}{c}{ Magnification } \\
\hline & $\begin{array}{c}\text { Sensor } \\
\text { width } \\
(\mathrm{mm})\end{array}$ & $\begin{array}{c}\text { Image } \\
\text { width } \\
\text { (pixels) }\end{array}$ & $\begin{array}{c}1 \times(\mu \mathrm{m} / \\
\text { pixel })\end{array}$ & $\begin{array}{c}2 \times(\mu \mathrm{m} / \\
\text { pixel })\end{array}$ & $\begin{array}{c}3 \times(\mu \mathrm{m} / \\
\text { pixel })\end{array}$ & $\begin{array}{c}4 \times(\mu \mathrm{m} / \\
\text { pixel })\end{array}$ & $\begin{array}{c}5 \times(\mu \mathrm{m} / \\
\text { pixel })\end{array}$ \\
Canon 5DS R & 36 & 8688 & 4.15 & 2.07 & 1.35 & 1.04 & 0.83 \\
Canon 700D & 22.3 & 5184 & 4.31 & 2.15 & 1.43 & 1.08 & 0.86 \\
\hline
\end{tabular}

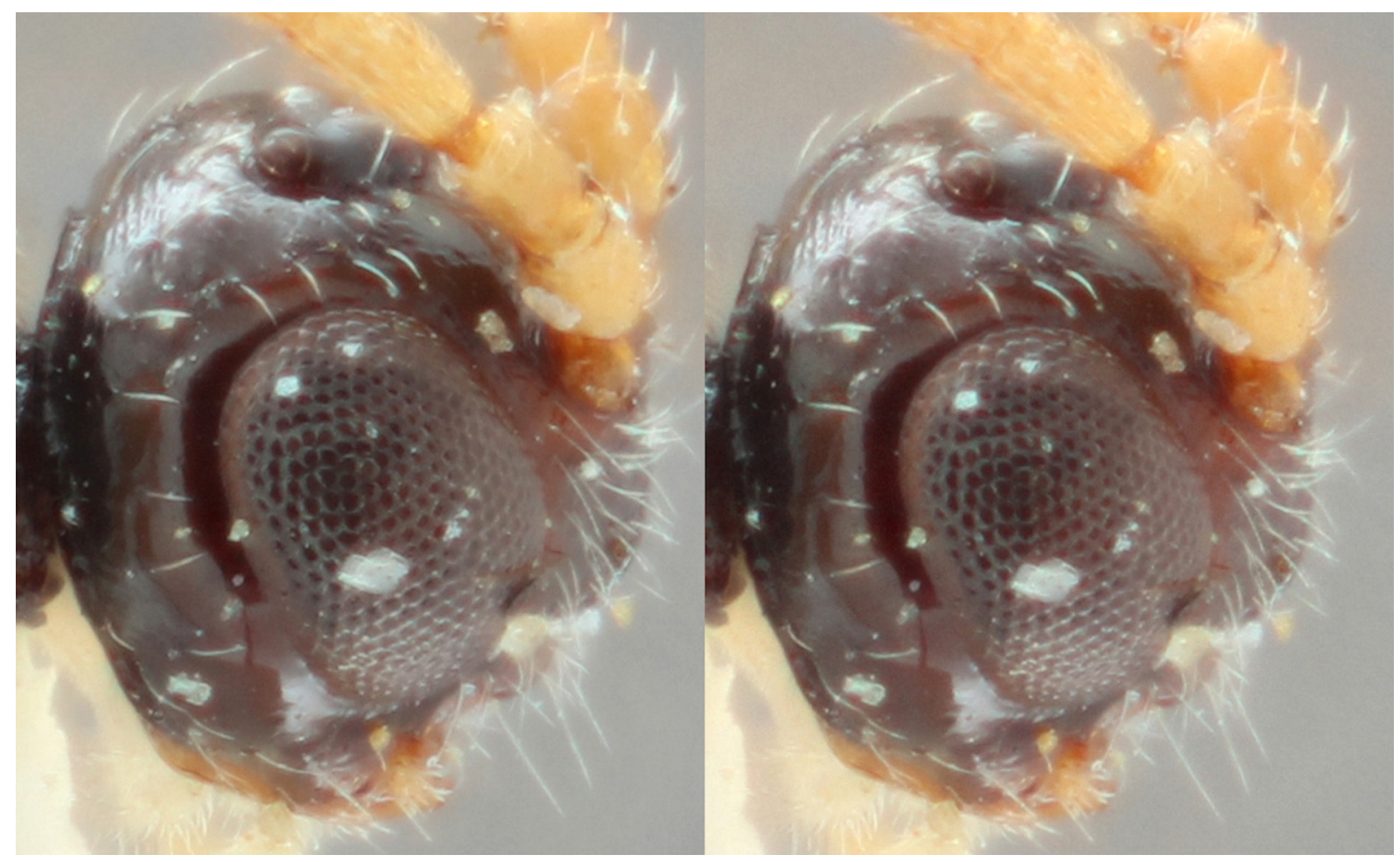

Fig. 2.2. Visual camera sensor comparison: A Hormius sp. specimen pictured at $4 \times$ magnification with a Canon EOS 5DS R (left) and a Canon EOS 700D (right).

\subsubsection{Collections}

The main goal of using the focus stacking set-up within the Belgian digitisation project (DIGIT-03) is to provide highly detailed pictures of type specimens that are too small to digitise in $3 \mathrm{D}$ without the use of a $\mu \mathrm{CT}$ scanner.

In essence the specimens together with their challenges can be divided according to their way of storage: 
wet or dry. The wet collection mainly houses specimens preserved in ethanol, while the dry collection are unattached or attached specimens (pinned, glued, on a microscope plate).

Aside from the storage medium, within each group various other difficulties might occur which are related to the specimen's appearance: hairy, shiny, translucent, etc. All museum collections are suitable (Geology, Anthropology, Palaeontology, Entomology, Vertebrates, Recent Invertebrates, Plants, ...) for specimens of $2 \mathrm{~mm}$ to $20 \mathrm{~cm}$, wether they are stored wet or dry.

\subsubsection{Dry Collections}

Shiny, metallic specimens

Minerals can be quite tricky to picture as they often have crystal faces that reflect light when lit under a certain angle. When trying to prevent such reflection from happening, shadows are often created which can obscur other interesting parts. However, as the pictures of Fig. 2.3 show, a very low amount of reflection is apparent and hardly any shadows are created; the entire specimen is in focus thanks to the focus stacking technique.

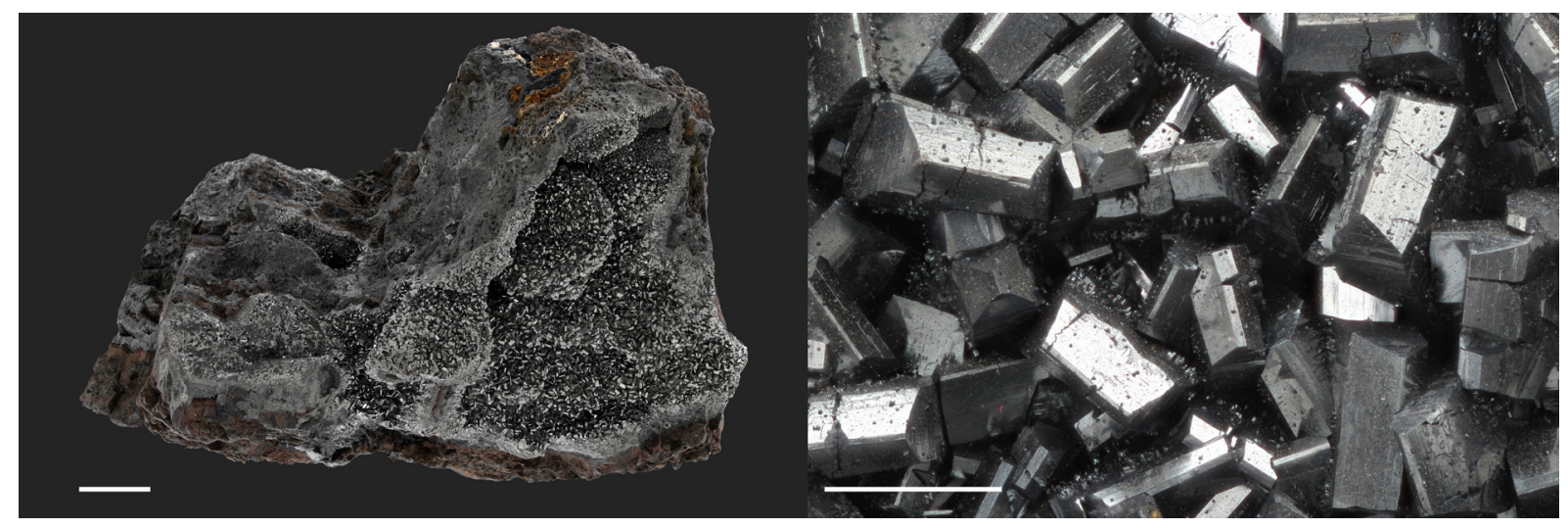

Fig. 2.3. Picturing shiny metallic specimens: Left: A manganite specimen $(\mathrm{scale}=1 \mathrm{~cm})$; Right: a detailed view of the manganite specimen $($ scale $=1 \mathrm{~mm})$.

\section{Pinned specimens}

Within the group of pinned and glued specimens the main issue is the possible reflection of light on the specimen itself or on the paper it is attached to. With a regular light system, shadows are often created or parts become overexposed due to the white labels routinely attached to a specimen. Focus stacking utilises diffuse lighting, meaning that reflection rarely appears, even on highly reflective surfaces like the elytra of beetles or the wings of insects (Figs 2.4-2.5). To picture pinned insects an insect manipulator is used, which keeps the insect centered when different positions need to be pictured. At least the dorsal and lateral side are pictured, often the frontal view as well or more characteristics depending on the specimen or digitisation request.

\section{Detached specimens}

Although many specimens are positioned using a pin, some are also stored freely within tubes, boxes, etc. Positioning such specimens can be the most challenging part of taking pictures. In such cases, the specimen can be placed onto a small transparent plate (e.g., an acrylic or glass petri dish). It is important to create some distance between the specimen and the background to avoid background details in the picture. A black, grey or white background is preferable to make the specimen stand out (Figs 2.6-2.7). 


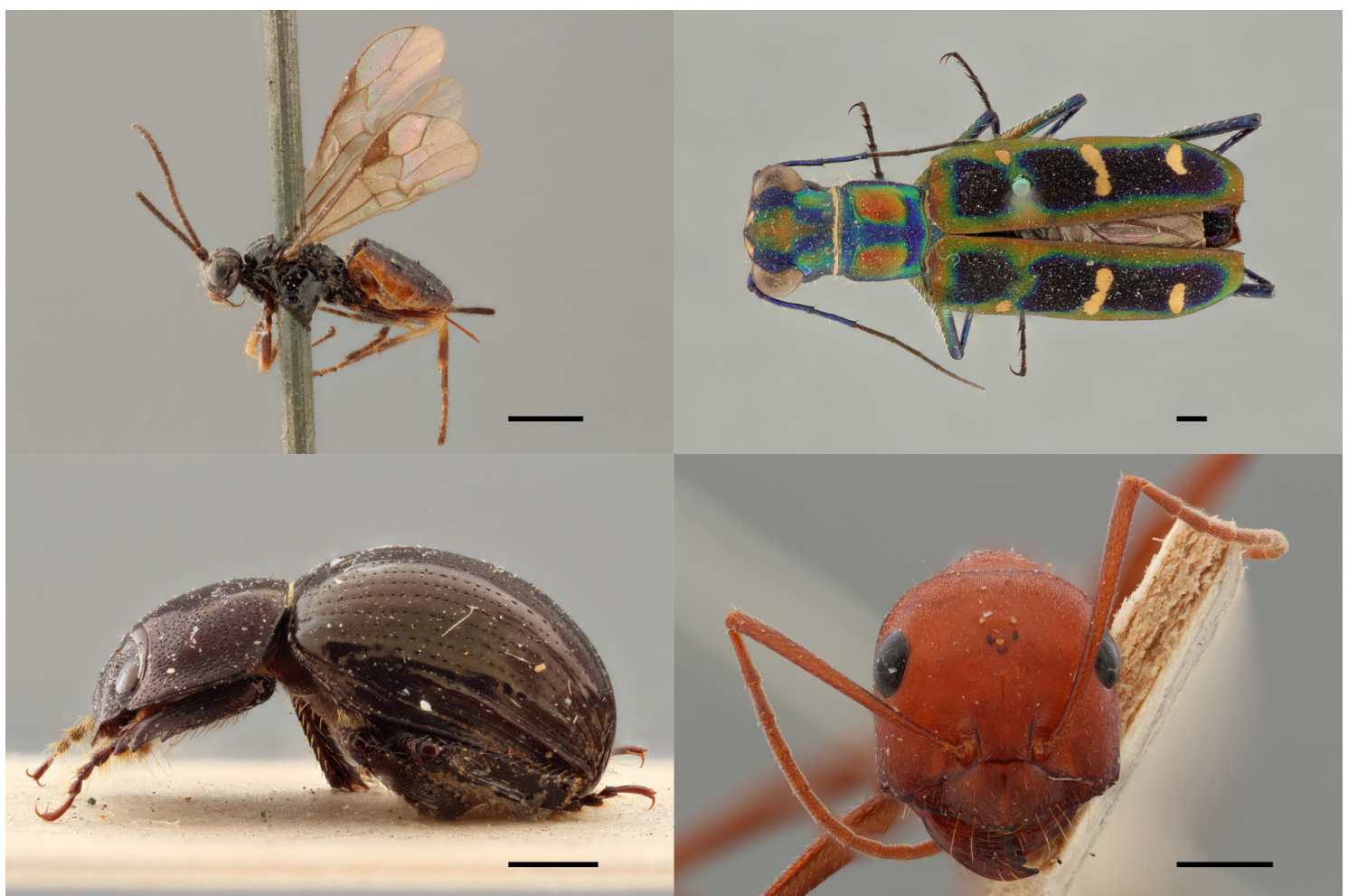

Fig. 2.4. Picturing pinned insects. The pictures represent several insects with highly reflective surfaces positioned in different ways (pinned, upper pictures; glued, bottom pictures). No shadows, no overexposed areas and hardly any reflections are present. Scale $=1 \mathrm{~mm}$.

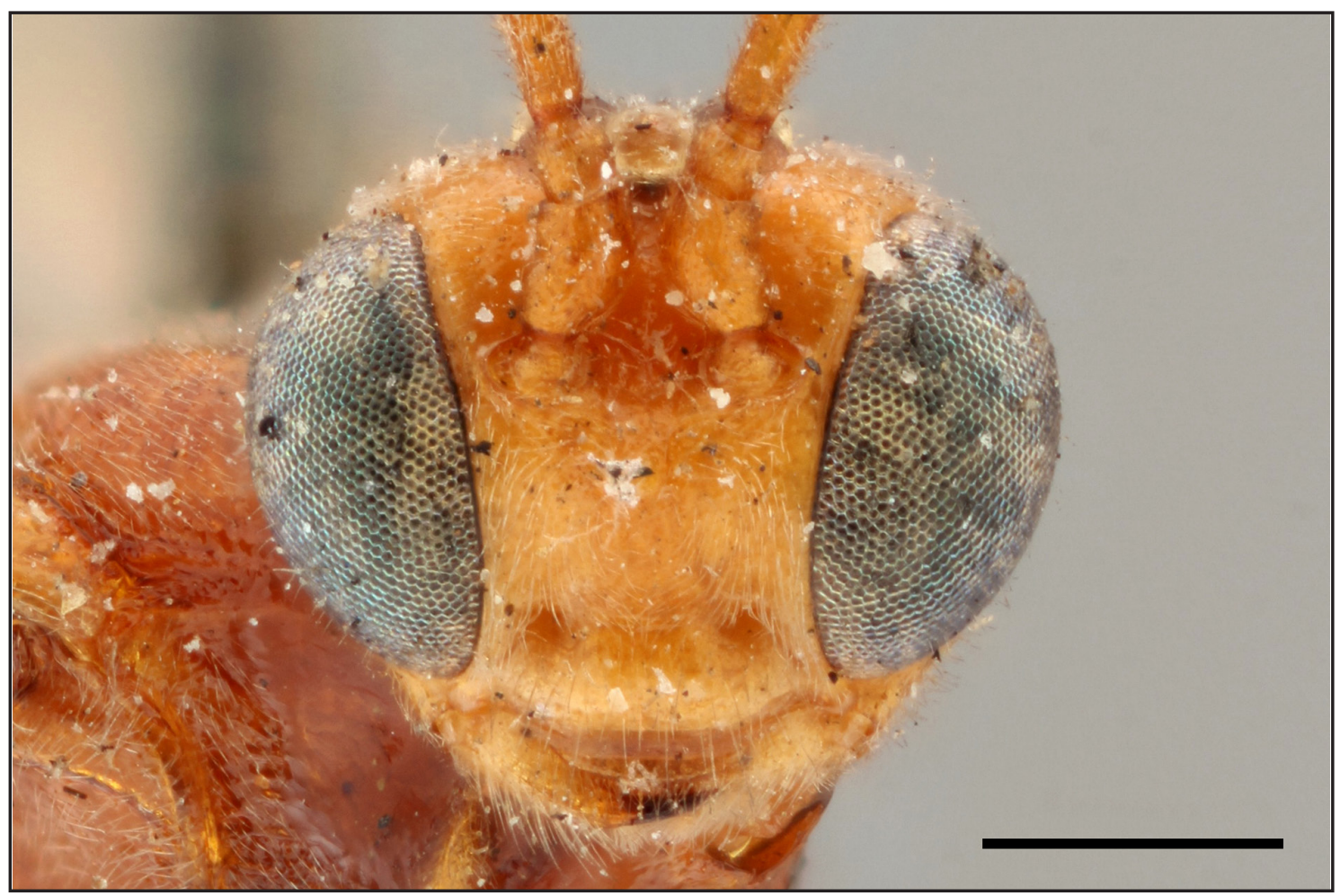

Fig. 2.5. Detail of pinned insect image. Frontal view of a specimen of Bracon chrysophthalmus at $5 \times$ magnification. Scale $=500 \mu \mathrm{m}$. 
A small piece of plasticine can be used to hold the specimen in the desired position. However, sometimes the specimens are too small or too fragile to push them into the plasticine. It was discovered by accident that after cleaning acrylic petri dishes with a microfiber cloth for cleaning lenses, the surface becomes statically charged. At first this seemed to complicate positioning the specimen, but by using gentle movements it is often possible to place it in the perfect position, due to the statically charged surface, even when the contact area is very small. Of course, this only works for small and light specimens.

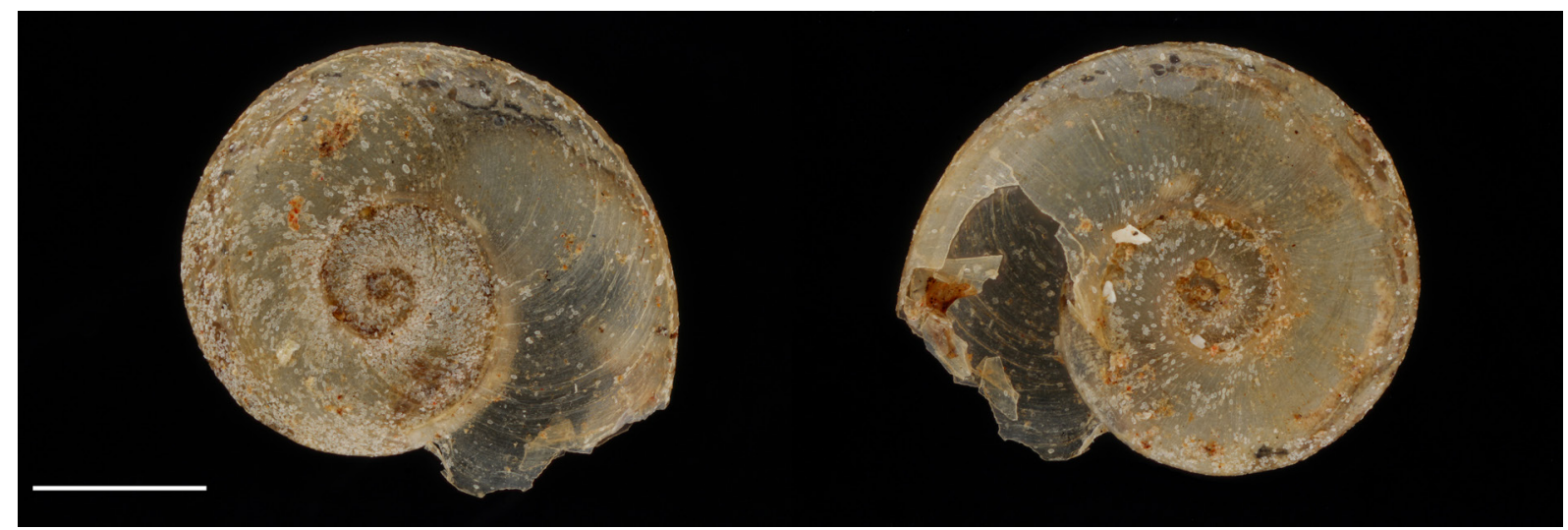

Fig. 2.6. Translucent specimens. Pictures of a small translucent specimen of Gyraulus costulates exilis. Scale $=1 \mathrm{~mm}$.

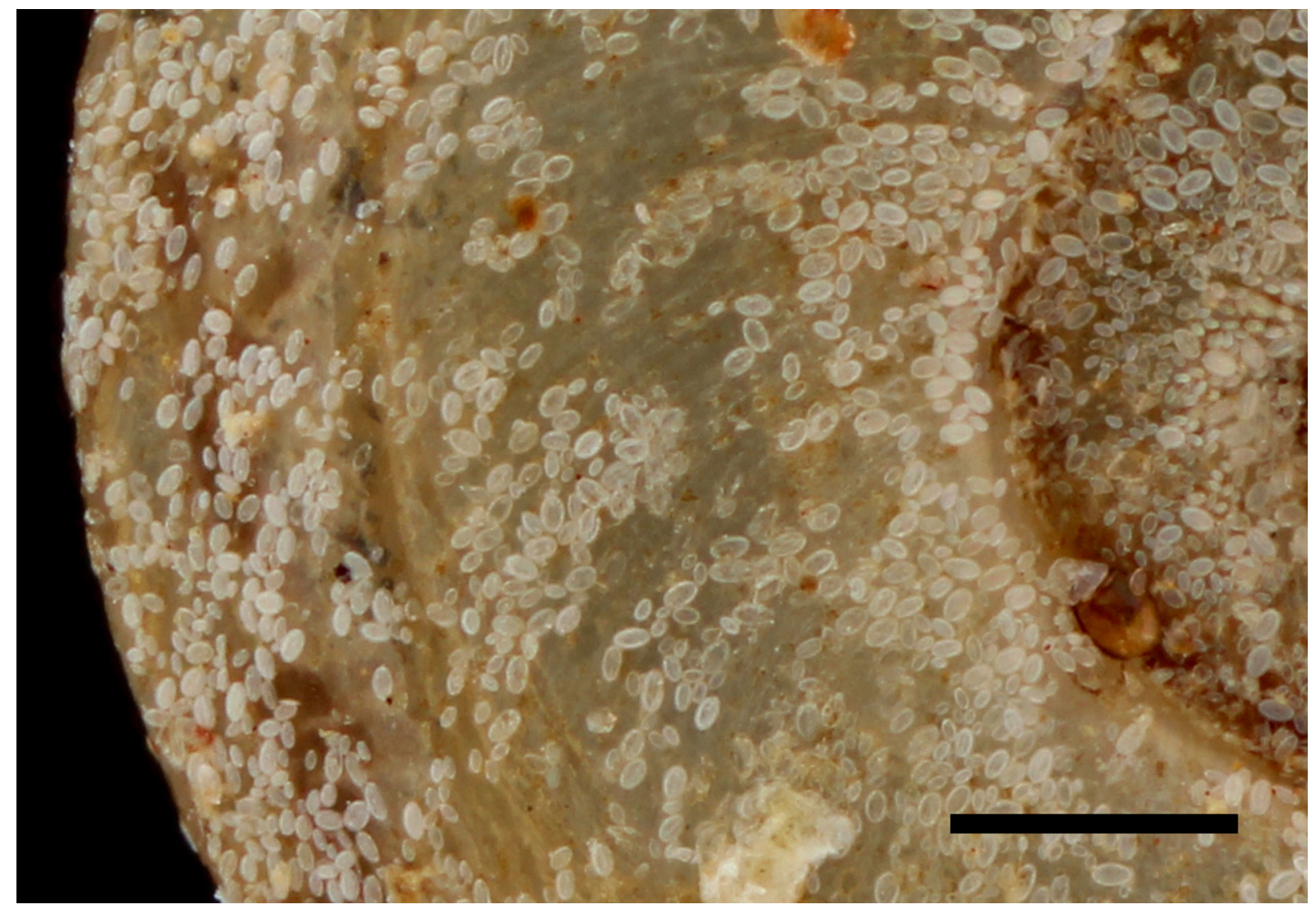

Fig. 2.7. Translucent specimen in detail. Close-up view of the outer surface of a specimen of Gyraulus costulates exilis. Scale $=500 \mu \mathrm{m}$. 
Microscope slides

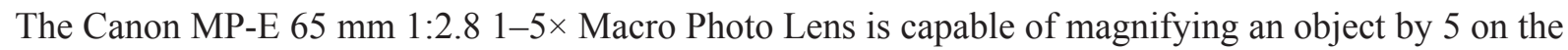
sensor of the camera. It is therefore perfectly possible to use it for taking pictures of certain specimens placed on microscope slides. The benefit with this system is that diffuse light comes from any direction onto the slide, making it possible to switch backgrounds and to enhance certain features (Fig. 2.8).
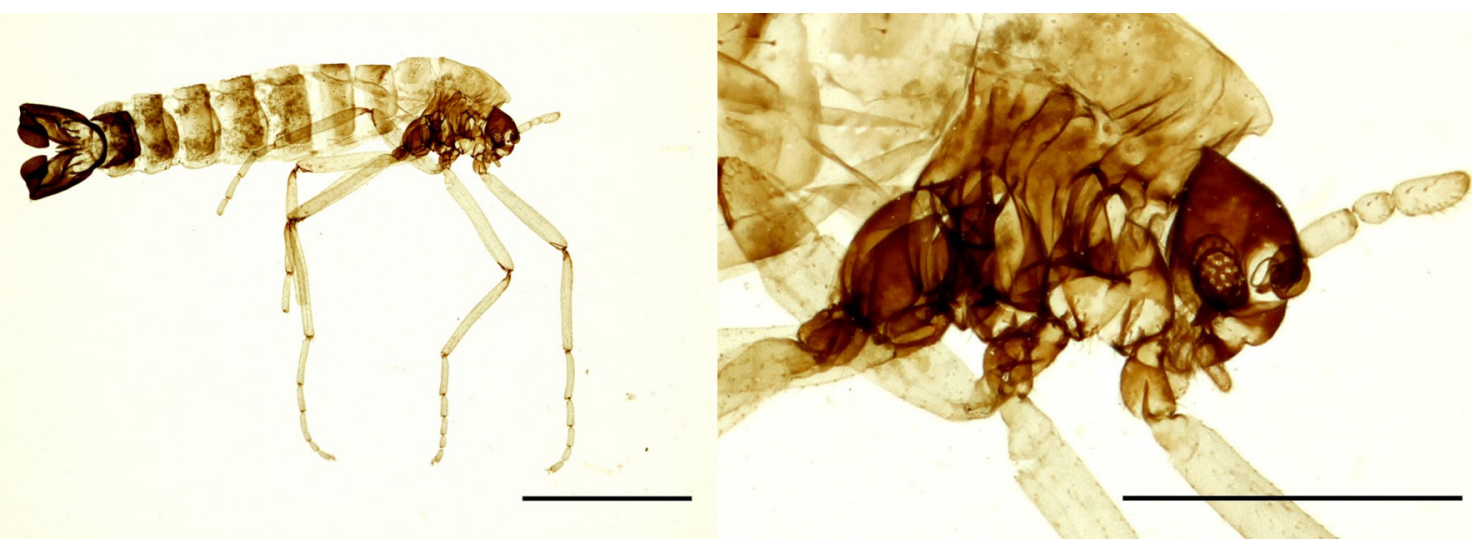

Fig. 2.8. Pictures of a microscope plate. Left: a microscope plate with an overview of a specimen of Belgica antarctica using a white background (scale $=1 \mathrm{~mm}$ ). Right: a detailed view of the left image $($ scale $=500 \mu \mathrm{m})$.

\subsubsection{Wet collections}

Specimens in wet collections are generally stored in an ethanol solution to prevent desiccation. Because of this preservation technique, it is important that the specimens remain within their storage solution, even when taking pictures. Aside from different backgrounds that can be used while taking pictures, glass beads can be placed within the container holding the specimen to prevent movement. The test specimen, a fly of the genus Drosophila was pictured submerged in ethanol $(70 \%)$ in a petri dish, with and without glass beads (Fig. 2.9, detail in Fig. 2.10). This small container (petri dish) was positioned on another upside-down petri dish, which was then positioned on grey and white pieces of paper. It is important that both the specimen and the ethanol are as free of dust and dirt as possible in order to obtain aberrant free backgrounds (Fig. 2.9). It is also possible to remove any unwanted dust or dirt particles afterwards using digital editing tools.

Aside from specimens submerged in ethanol or formaldehyde solutions, some specimens are kept in other media. Glycerin is such a medium that is used to store cleared and stained specimens, a technique to render bone and cartilage visible for morphological studies. These specimens are very fragile after this particular treatment and it would be necessary to remove them from their container to study them closely unless good pictures are available, such as those shown in Fig. 2.11. This was shot in an old acrylic holder, so the scratches are visible on the background of the pictures.

The versatility of the white closet with the mounted StackShot and the flash positioned below the specimen to be pictured cannot be overstated. Previous studies (Brecko et al. 2014) have shown that this setup is able to compete with high-end stacking solutions, delivering better and more detailed pictures at certain magnifications for only a tenth of the price (around 3000 euros including a workstation). It is possible to save some money on the complete kit by changing the MP-E $65 \mathrm{~mm} \mathrm{f} / 2.81-5 \times$ Macro Photo with the Laowa $25 \mathrm{~mm}$ f/2.8 2.5-5× Ultra Macro (Venus Lens 2018) which, according to the first 

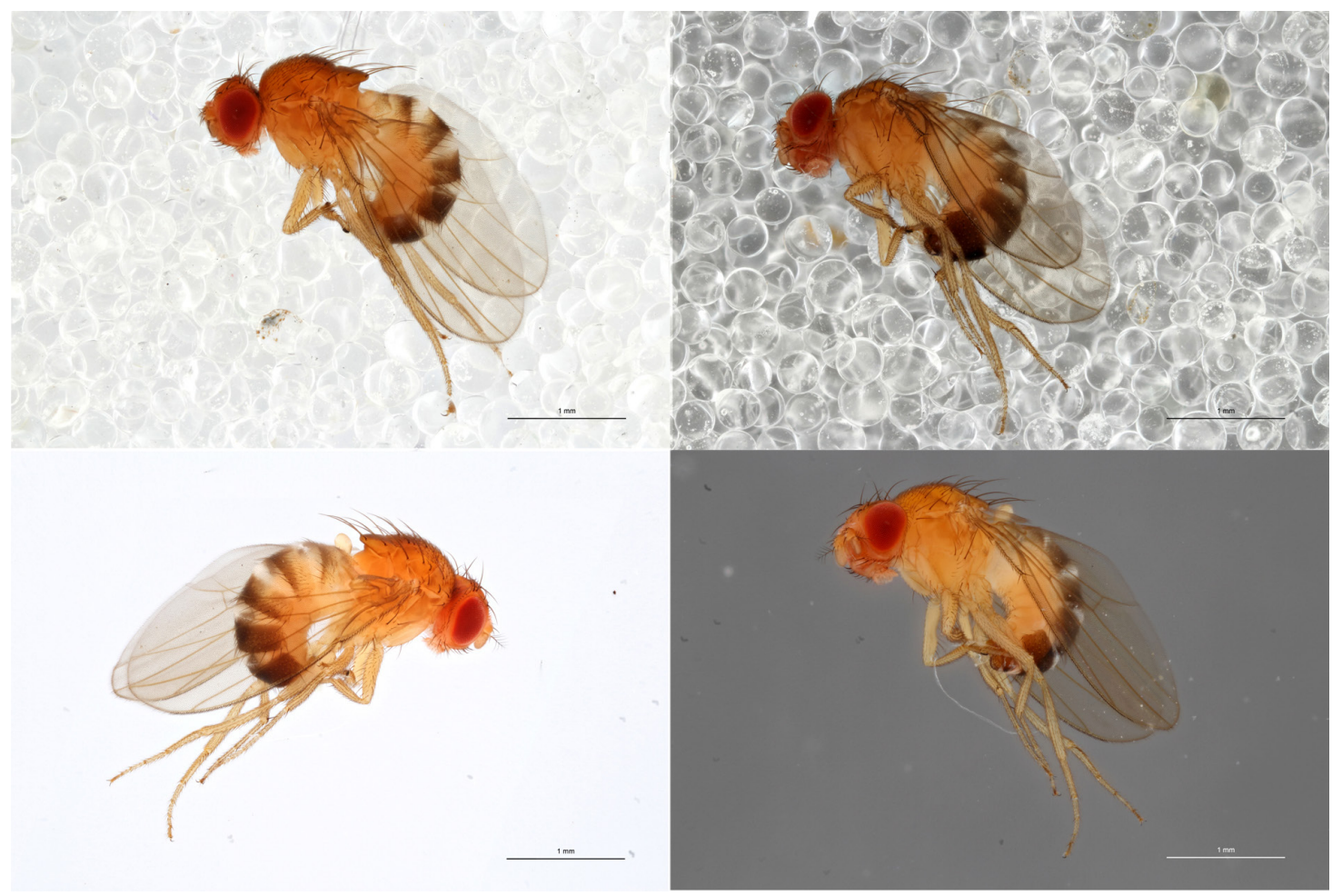

Fig. 2.9. Picturing submerged specimens. A fly of the genus Drosophila pictured in ethanol with glass beads (top pictures) or without (bottom pictures) using a white (left pictures) or grey (right pictures) background. Scale $=1 \mathrm{~mm}$.

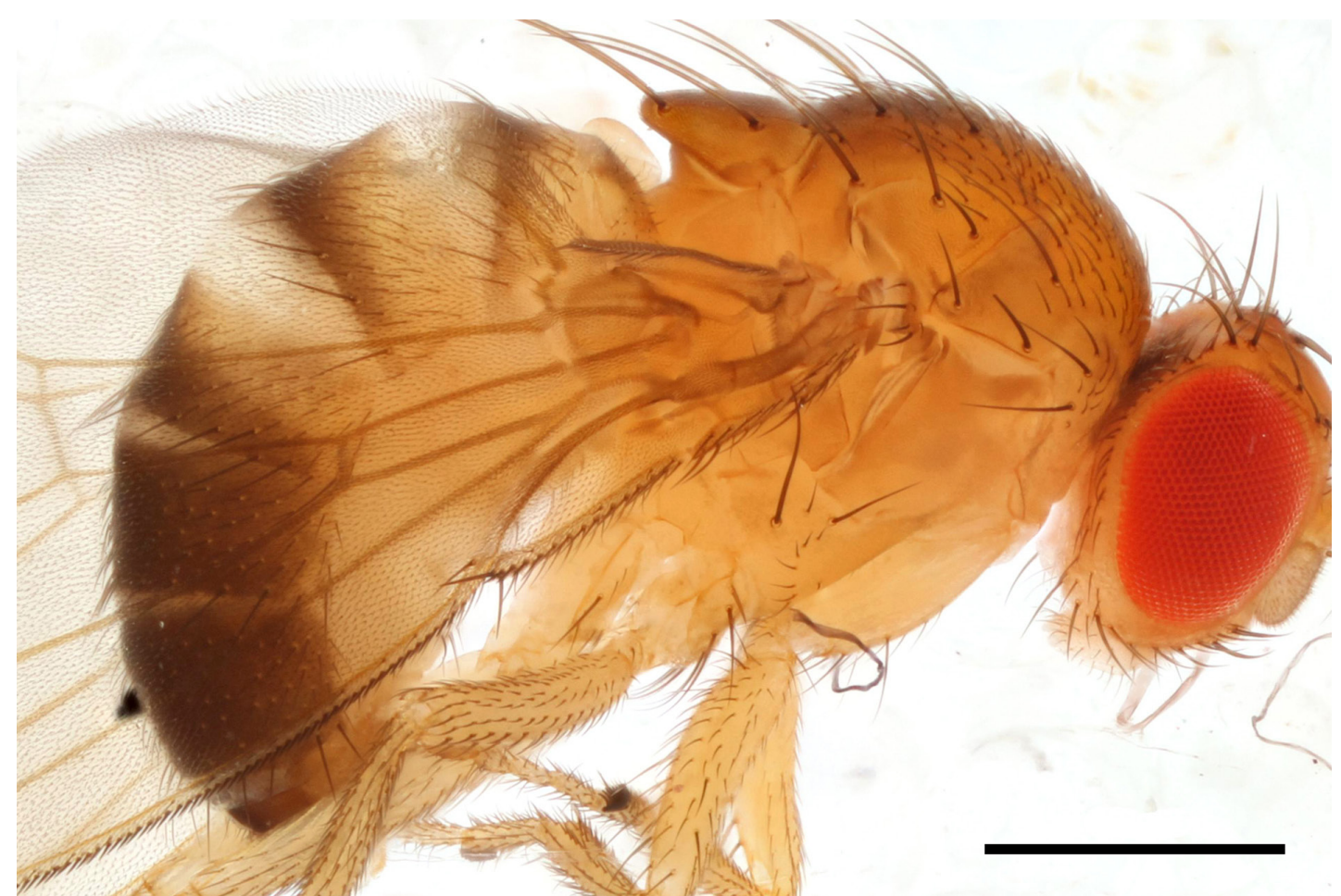

Fig. 2.10. Detail of a specimen stored in ethanol. A detailed lateral view of a Drosophila sp. Specimen submerged in a $70 \%$ ethanol solution. Scale $=500 \mu \mathrm{m}$. 


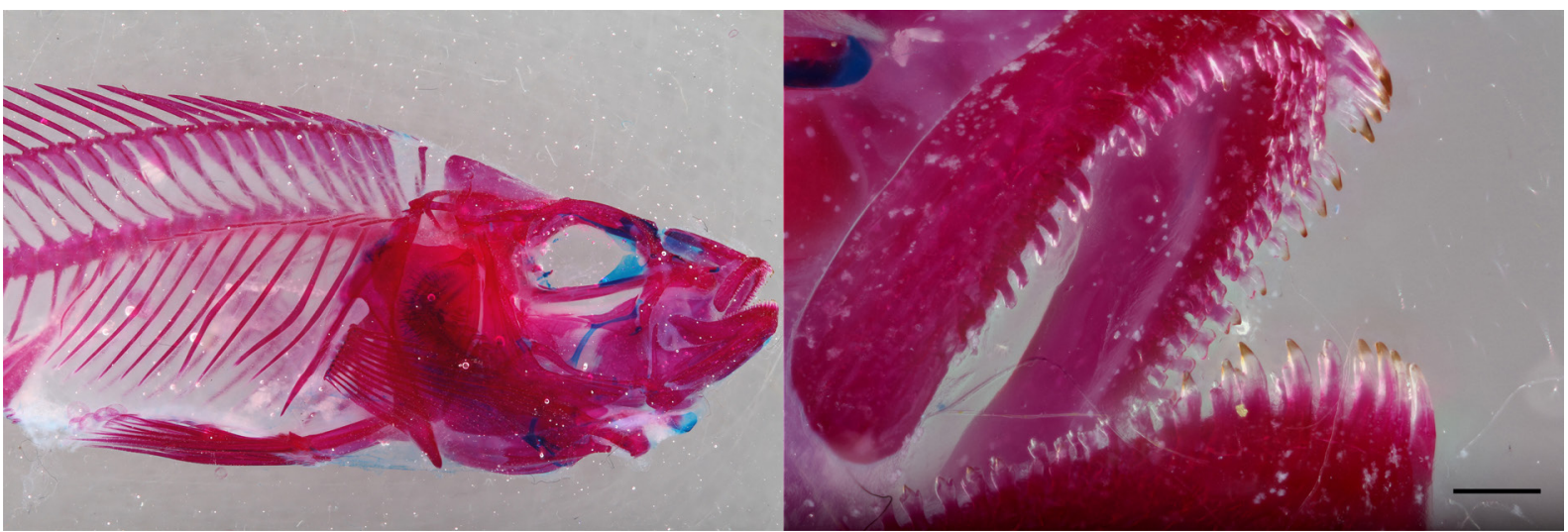

Fig. 2.11. Specimen stored within glycerin. Cleared and stained specimen of Haplochromis sp. pictured in glycerin. The right part of the picture is photographed with a magnification of $5 \times$. Scale $=500 \mu \mathrm{m}$.

online tests (Bay 2018; Wizen 2018), proved to deliver equal quality for half of the price. A lot of the small specimens inside a museum collection can be pictured with ease using focus stacking, as the light system creates an evenly lit specimen even within media like ethanol or glycerin. This is quite beneficial as specimens stored within fluids are rarely covered within digitisation programs.

There are no apparent issues with imaging translucent, transparent or reflective specimens, regardless of their size. An extra benefit, especially when picturing microscope plates, is the possibility to change the background and create different contrasts to enhance details of the specimen's morphology.

Besides the use in the framework of the Belgian digitisation project, DIGIT-03, the set-up is used by scientists at our institutes to picture newly described species, taking detailed pictures of the whole specimen or certain morphological features when needed for publications. Pictures, in full resolution, taken of type specimens in the framework of DIGIT-03 for Royal Belgian Institute of Natural Sciences can be studied at the virtual collections web page (http://virtualcollections.naturalsciences.be/) and the DIGIT-03 webpage of the Royal Museum for Central Africa (http://digit03.africamuseum.be).

\subsubsection{References}

Bay N. 2018. Laowa $25 \mathrm{~mm} \mathrm{f} / 2.8$ 2.5-5× Review: Ultra Macro Lens Comparison with Canon MPE65. Available from https://www.nickybay.com/2018/03/laowa-25mm-f28-25-5x-review-ultramacro.html [accessed 24 Jul. 2018].

Dupont S., Price B. \& Blagoderov V. 2015. Imp: the customizable LEGO Pinned Insect Manipulator. Zookeys 481: 131-138. https://doi.org/10.3897/zookeys.481.8788

Brecko J., Mathys A., Dekoninck W., Leponce M., VandenSpiegel D. \& Semal P. 2014. Focus stacking: comparing commercial top-end set-ups with a semi-automatic low budget approach. A possible solution for mass digitisation of type specimens. Zookeys 464: 1-23. https://doi.org/10.3897/zookeys.464.8615

Hess M. \& Robson S. 2013. Re-engineering Watt: A case study and best practice recommendations for 3D colour laser scans and 3D printing in museum artefact documentation. In: Saunders D., Strlic M., Kronen C., Birholzerberg K. \& Luxford N. (eds) Lasers in the Conservation of Artworks IX: 154162. Archetype Publications, London. Available from https://discovery.ucl.ac.uk/id/eprint/1411525/ [accessed 30 Mar. 2020]. 
Hess M., Robson S. \& Hosseininaveh Ahmadabadian A. 2014. A contest of sensors in close range 3D imaging: Performance evaluation with a new metric test object. The International Archives of the Photogrammetry, Remote Sensing and Spatial Information Sciences 5: 277-284. https://doi.org/10.5194/isprsarchives-XL-5-277-2014

Jecic S. \& Dvar N. 2003. The assessment of structures light and laser scanning methods in 3D shape measurements. $4^{\text {th }}$ International Congress of Croatian Society of Mechanics: 237-244.

Mallison H., Hohloch A. \& Pfretzschner H.-U. 2009. Mechanical digitising for paleontology - new and improved techniques. Palaeontologia Electronica. 12 (2): 4T.: 1-41.

http://palaeo-electronica.org/2009_2/185/index.html

Mallison H. 2011. Digitising methods for paleontology - applications, benefits and limitations. In: Elewa AMT (ed.) Computational Paleontology: 7-44. Springer, Berlin.

Mathys A., Brecko J. \& Semal P. 2013a. Comparing 3D digitising technologies: what are the differences? In: Addison A.C., De Luca L., Guidi G. \& Pescarin S. (eds) Digital Heritage International Congress, Vol. 1: 201-204. CNRS, Marseille. https://doi.org/10.1109/DigitalHeritage.2013.6743733

Mathys A., Brecko J., Di Modica K., Abrams G., Bonjean D. \& Semal P. 2013b. Low cost 3D imaging: a first look for field archaeology. Notae Praehistoricae 33: 33-42.

Mathys A., Brecko J. \& Semal P. 2014. Cost evaluation of 3D digitisation techniques. In: Ioannides M., Magnenat-Thalmann N., Fink E., Zarnic R., Yen A. \& Quak E. (eds) EUROMED 2014 Proceedings: 17-25. Multi-Science Publishing Co., Hockley.

Mathys A., Brecko J., Vandenspiegel D. \& Semal P. 2015. 3D and challenging materials: guidelines for different 3D digitisation methods for museum collections with varying material optical properties. Proceedings of the $2^{\text {nd }}$ International Congress on Digital Heritage.

https://doi.org/10.1109/DigitalHeritage.2015.7413827

Venus lens. 2018. Laowa $25 \mathrm{~mm}$ f/2.8 2.5-5× Ultra Macro.

Available from https://www.venuslens.net/product/laowa-25mm-f-2-8-2-5-5x-ultra-macro-2/ [accessed 24 Jul. 2018].

Wizen G. 2018. Review: Laowa $25 \mathrm{~mm} \mathrm{f} / 2.8$ 2.5-5× Ultra Macro lens. Available from http://gilwizen.com/laowa-25mm-ultra-macro-lens-review/ [accessed 24 Jul. 2018]. 


\subsection{D+ Focus Stacking (Macro/Micro) Microscope}

Many specimens in natural history collections are small and/or parts of them are mounted on microscope slides. Although it is an old technique, the past decades have brought microscopy into the digital world (Taylor 2011). If the mounted part or specimen is larger than a few millimeters, it is possible to picture it using the focus stacking setup described previously (Chapter 2, part 2.1). However, if the specimens are small, i.e., less that 2-3 mm, or a quick gigapixel-resolution image consisting of high detailed images at high magnifications is necessary, then a microscope with mounted digital camera is the way to proceed (Leong \& McGee 2001; Higgins 2015). Many different models exist, of which the most common ones are from brands like Leica, Olympus, Nikon and Zeiss, each with their corresponding software, but usually all work more or less the same (Rojo et al. 2006). In both RBINS and in RMCA, two existing microscopes were refurbished with a motorized stage to allow automatic X-Y stitching or Z-stacking, or a combination of both. Because of the X-Y stitching it is possible to picture a specimen, or parts of it, at a higher magnification, which is necessary to identify all the important structures mounted on a slide. If the specimen is thicker than the depth of field provided by the microscope lens an additional Z-stack can produce the extended depth of field needed to see all the structures in focus. This means that once digitised, a slide can be viewed at the desirable magnification with additional focal depth to see the complete specimen in one sharp view. After this process it is often a lot easier to identify the important information present on a slide.

\subsubsection{Material and methods}

'Information in Images' motorized stage, motorized focus adapter and a QiCam colour camera of 1392 $\times 1040$ pixels, sensor with $4.65 \times 4.65$ micron pixels, 12 -Bit colour output. This can be mounted on almost any type of microscope (Figs 2.12-2.13).

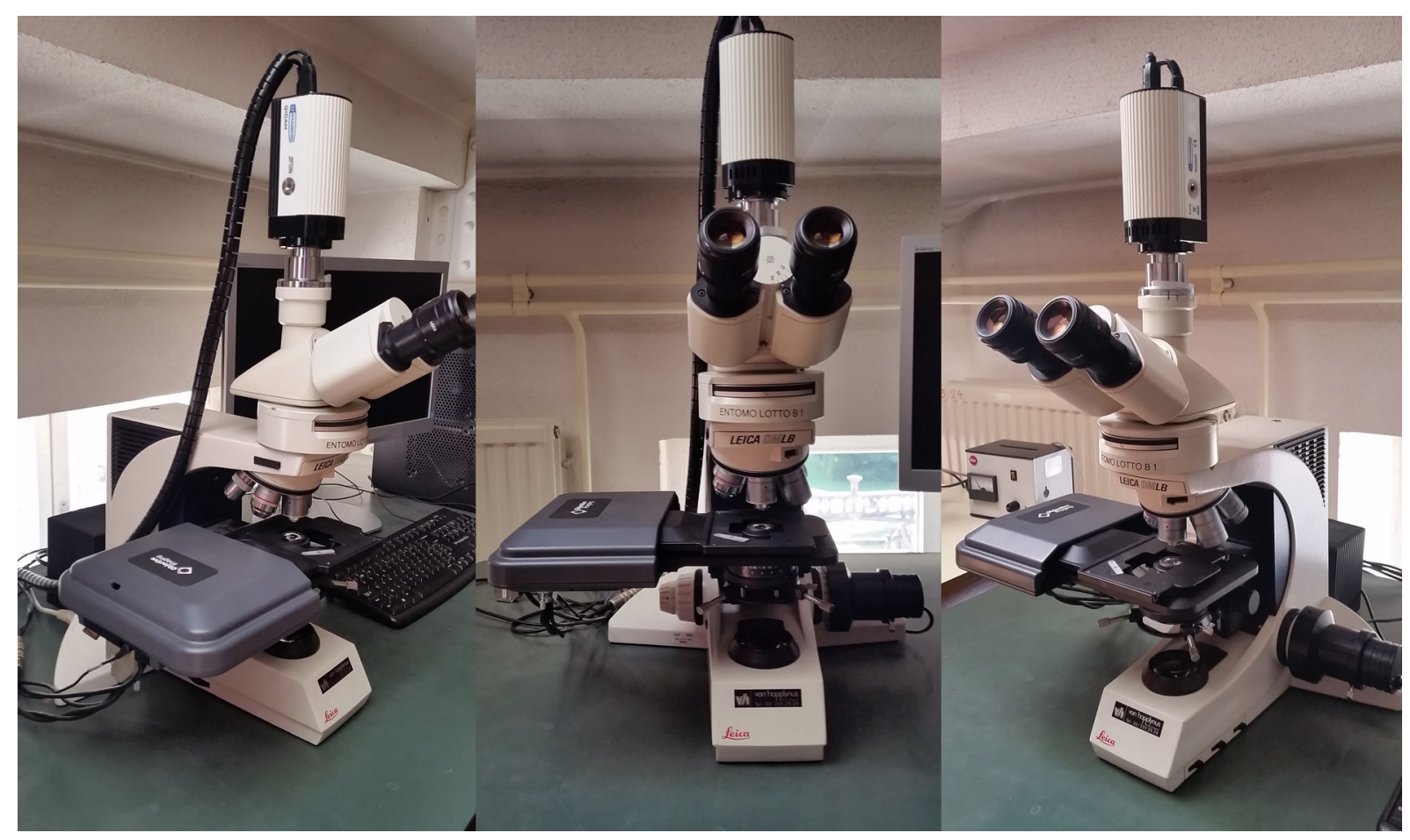

Fig. 2.12. General view of the 'Information in Images' system mounted on a Leica microscope at the Royal Museum for Central Africa. 

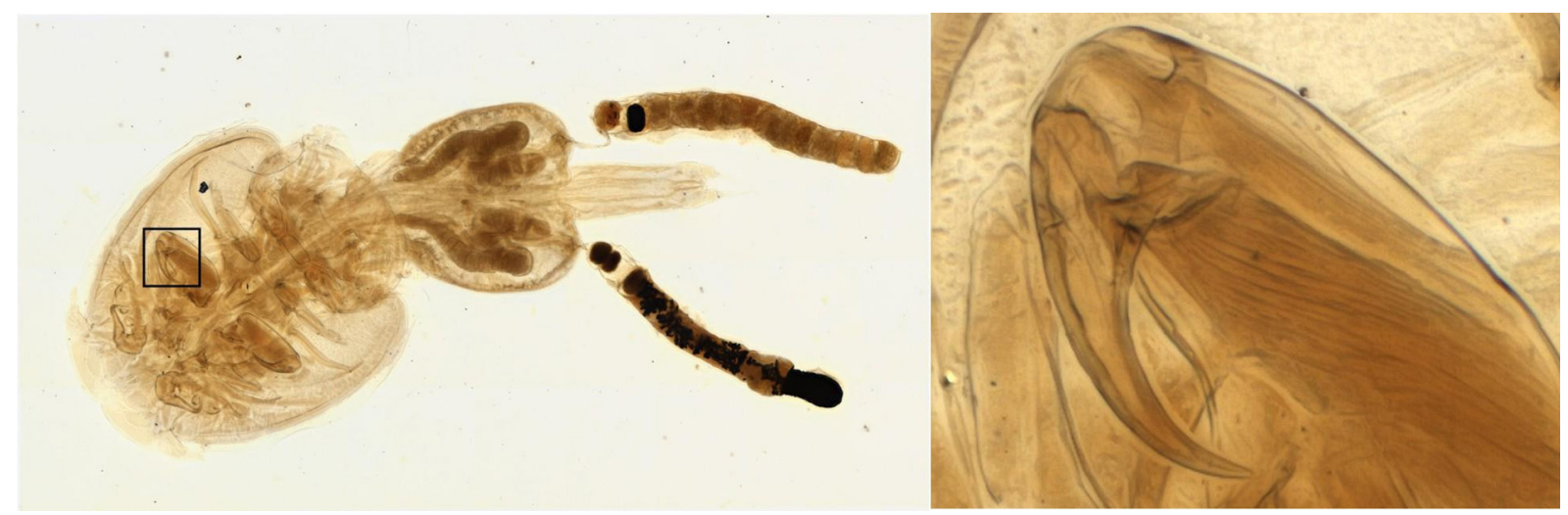

Fig. 2.13. Picture of a copepod composed of several stitched tiles of stacked pictures at high magnification. The left-hand picture shows the overall view of the composite image. The zone covered by the square is the part visible on the right-hand picture, which shows the detail of the specimen.

The microscope is able to make both focus stacks and gigapixel-resolution images, as with a SatScan system (Blagoderov et al. 2012) or D-Scan system (Schmidt et al. 2012), but with the precision and magnification of a microscope. In this way it is possible to zoom substantially on a microscope image.

Besides the ability of the system to scan a microscope slide with enhanced resolution in 2D (X-Y stitching and Z-stacking), the key feature of the system is the speed at which it delivers the final image. It is possible to scan a slide containing an histological section of a mouse at $10 \times$ magnification, combining 338 images in the mosaic picture in 41s (Objective Imaging 2009; anonymous 2014; Information in images 2018). In the software (Surveyor) it is possible to draw a region of interest which one wants to have scanned (Fig. 2.14). The software automatically calculates the number of pictures needed to image the selected part at the set magnification. In case the depth of field is not sufficient it is possible to take

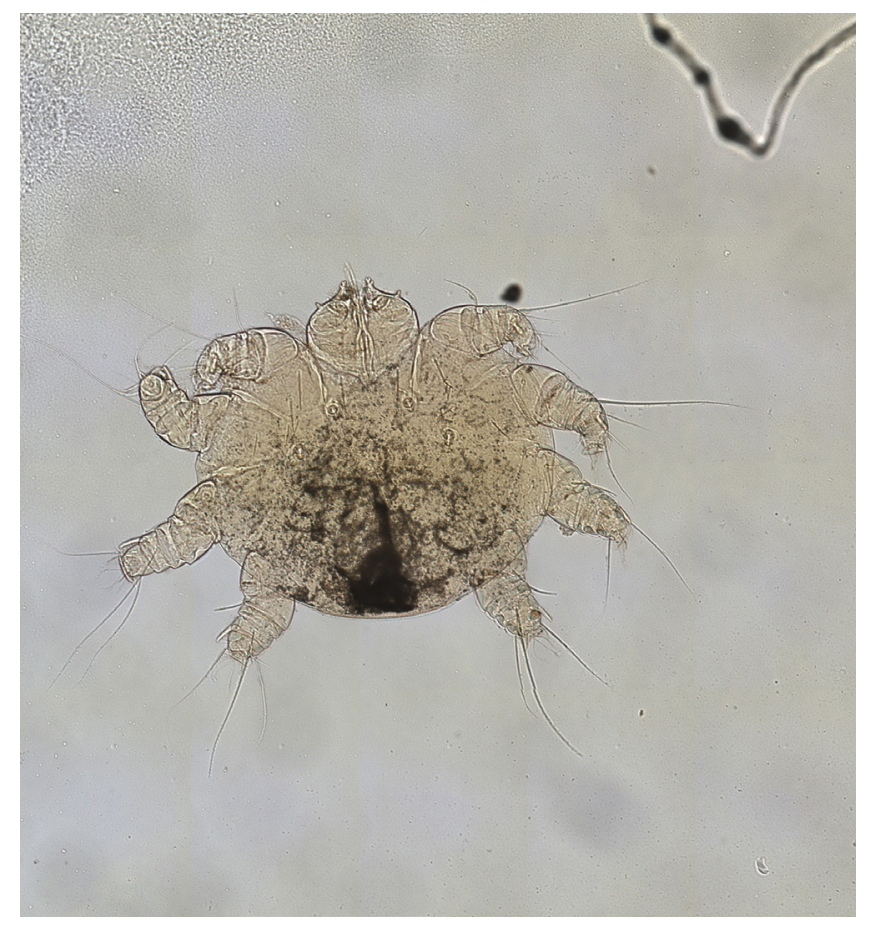

Fig. 2.14. Picture of a quickly composed image of a mite at $20 \times$ magnification. 
multiple pictures in the $\mathrm{Z}$ direction per tiled image. Afterwards it is possible to save the picture(s) in different ways: as a stitched and stacked finished picture; as individually stitched layers but not stacked; as stacked tiles but not stitched; or all frames separately. The stitching algorithm in the software is very good, however Zerene Stacker (Zerene Systems 2017) is a lot better in stacking the different stitched layers. Therefore, at RBINS and RMCA the different X-Y stitched layers are saved and stacked in Zerene Stacker using the PMax function. In the Surveyor software it is also possible to directly perform measurements on and add scales to the produced image.

\subsubsection{Collections}

Any type of collection with specimens mounted on a microscope slide that can be visualised through bright-field microscopy is digitised with this system. For collection items like geological thin sections, sometimes the regular 2D+ system described above gives better results. Figs 2.13-2.16 show results of the $\mathrm{X}-\mathrm{Y}$ and $\mathrm{Z}$-stacked process.

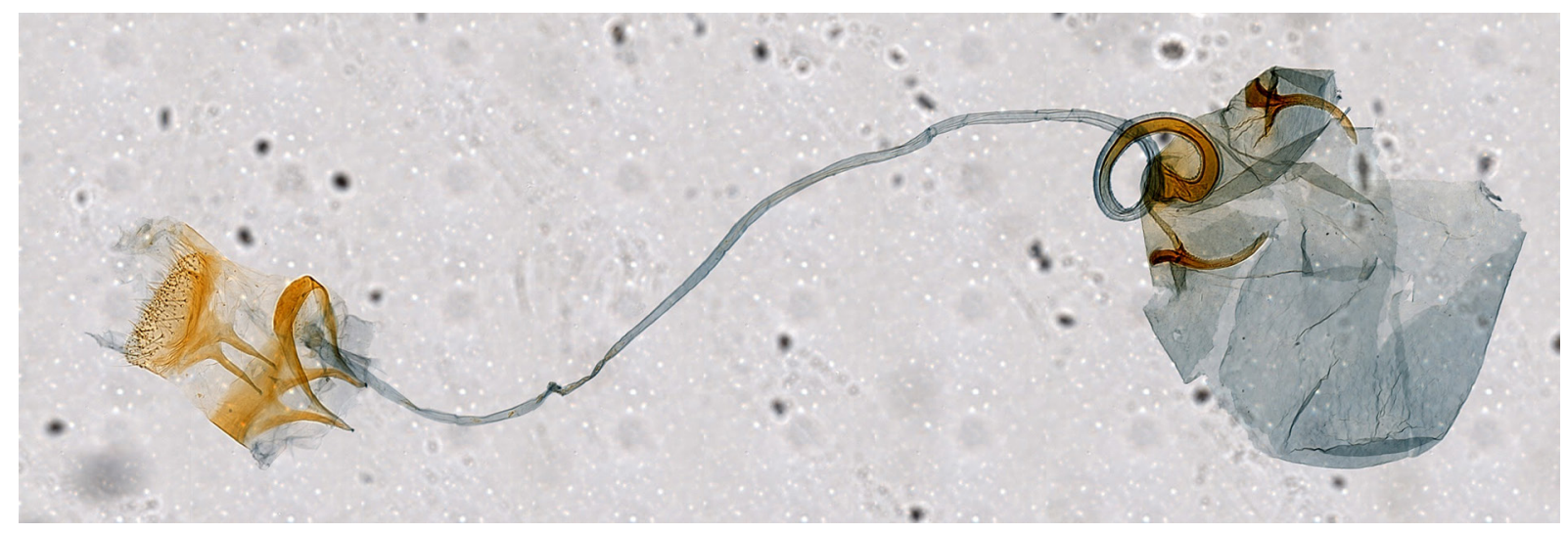

Fig. 2.15. Caloptilia galacotra, reproductive organs. Picture composed of several stitched tiles of stacked pictures at a magnification of $20 \times$.

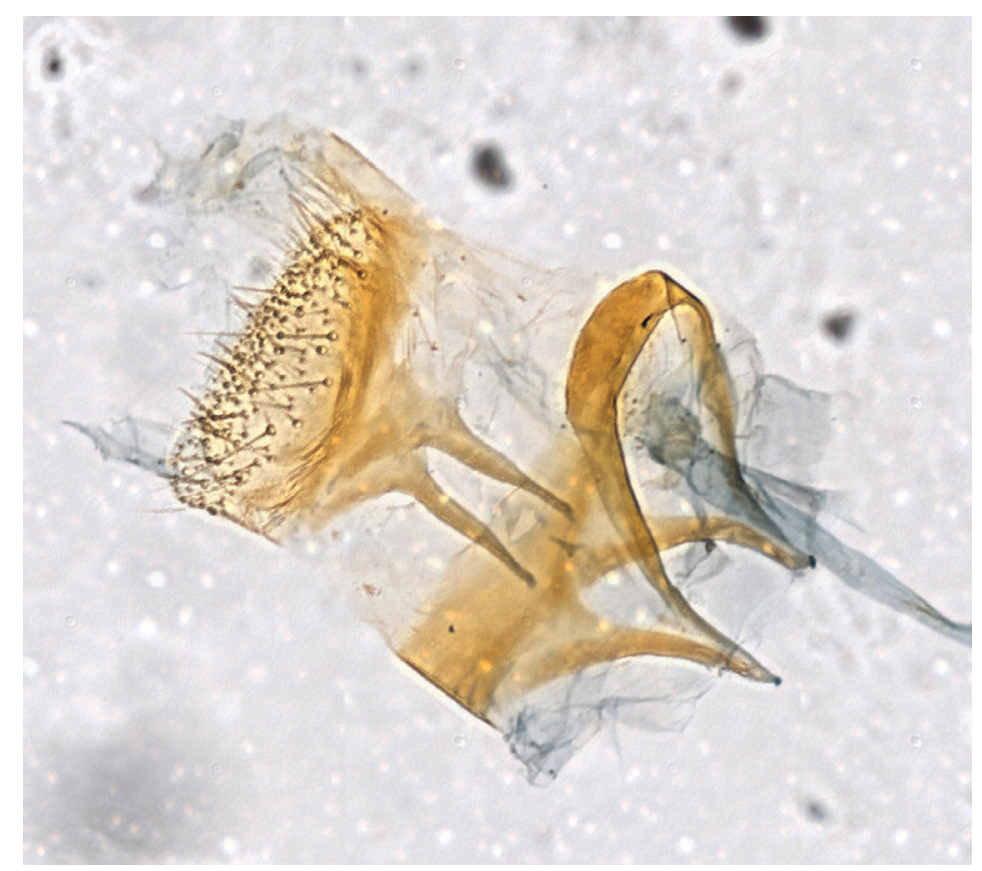

Fig. 2.16. Caloptilia galacotra, detail of the reproductive organs at $20 \times$ magnification. 
BRECKO J. \& MATHYS A., Best practice handbook for 2D+/3D digitisation

\subsubsection{References}

Anonymous. 2014. Microscope Slide Scanner Manual. Field Museum of Natural history.

Blagoderov V., Kitching I., Livermore L., Simonsen T. \& Smith V. 2012. No specimen left behind: industrial scale digitisation of natural history collections. ZooKeys 209: 133-146.

https://doi.org/10.3897/zookeys.209.3178

Higgins C. 2015. Applications and challenges of digital pathology and whole slide imaging. Biotechnic \& Histochemistry: Official Publication of the Biological Stain Commission 90 (5): 341-347.

https://doi.org/10.3109/10520295.2015.1044566

Information in Images. 2018. Available from https://www.informationinimages.com/auto-scan-micro [accessed 24 Jul. 2018].

Leong, F. J. \& McGee J. O. 2001. Automated complete slide digitisation: a medium for simultaneous viewing by multiple pathologists. The Journal of Pathology, 195 (4): 508-514.

https://doi.org/10.1002/path.972

Objective Imaging. 2009. Objective Imaging Surveyor Turboscan System with Slide Loader. Available from https://www.youtube.com/watch?v=RoHejrt-w9Q [accessed 24 Nov. 2019].

Rojo M.G., García G.B., Mateos C.P., García J.G. \& Vicente M.C. 2006. Critical comparison of 31 commercially available digital slide systems in pathology. International Journal of Surgical Pathology 14 (4): 285-305. https://doi.org/10.1177/1066896906292274

Schmidt S., Balke M. \& Lafogler S. 2012. DScan - a high-performance digital scanning system for entomological collections. ZooKeys 209: 183-191. https://doi.org/10.3897/zookeys.209.3115

Taylor C.R. 2011. From microscopy to whole slide digital images: a century and a half of image analysis. Applied Immunohistochemistry \& Molecular Morphology. 19 (6):491-493.

Zerene Systems. 2017. Available from http://zerenesystems.com/cms/stacker [accessed 24 Jul. 2018]. 


\subsection{ZooSphere and DISC3D}

The ZooSphere (2018) project has the backbone of the 2D+ system as its basis, but differs from the above by taking multiple $2 \mathrm{D}+$ images in an organized way. After each $2 \mathrm{D}+$ image, the specimen turns to the next position of a 360-degree set of images. After one rotation is completed the same is done for another inclination related to the original position of the specimen. In the end the 'camera positions' of the $2 \mathrm{D}+$ images form a sphere. The ZooSphere web-interface combines these images so a pseudo-3D can be consulted online. The major targets of the ZooSphere project are as follows:

- Provide an international repository and web hub for high-resolution image sequences of biological specimens

- Deliver content to various end-user devices, such as desktop computers, mobile devices and web browsers in general

- Create a tool for scientists, especially taxonomists, to speed up and improve their research

- Prevent physical object transfer via regular mail

- Reduce travel costs and efforts related to local object inspection

- Facilitate digital preservation of biological collection objects, which are subject to natural decay

- Increase the visibility and accessibility of biological collection objects

- Make objects available to both the general public and scientists

To meet these targets, Museum fur Naturkunde Berlin has designed a focus stacking system which enables to picture a specimen from every side automatically. In this way they end up with high resolution views of a specimen, which are transferred to a custombuilt viewer. Anyone is now able to view these scanned specimens at high resolution from different angles. A very nice part of their viewer is that the scale is always present on the image at any magnification. This helps both scientists and enthusiasts to get a clear view and distinguish key features of a certain species. Probably within the near future the software to control both the ZooSphere hardware as to distribute the pictures online will become available.

\subsubsection{Material and methods}

Two stepper motors are mounted is such a way that one is used to rotate the specimen along one axis and the other rotates both the previous stepper motor, with the specimen) along a perpendicular axis to the first rotation axis (Figs 2.17-2.18). These rotations allow the camera to capture the specimen from several angles. The first stepper motor stops $10-12$ times for a single $360^{\circ}$ rotation before the second stepper motor moves to another position. In total 100-144 views are taken for a single specimen in case of type specimens. These views are not single pictures, they are in fact focus stacks to enlarge the depth of field in one view. This is possible because the camera is positioned on a focus rail. The number of pictures taken per view depends on the specimen in front of the lens and is determined by the longest distance that needs to be covered to have the entire specimen in focus on that point. To ensure that the specimen is well lit from all sides, a large studio flash light with a soft box is used. The light is diffused and stable because it is powered directly by the mains. A series of styrofoam walls is built around the specimen, this reflects the light onto the specimen and lights every aspect of it (Fig 2.19). 


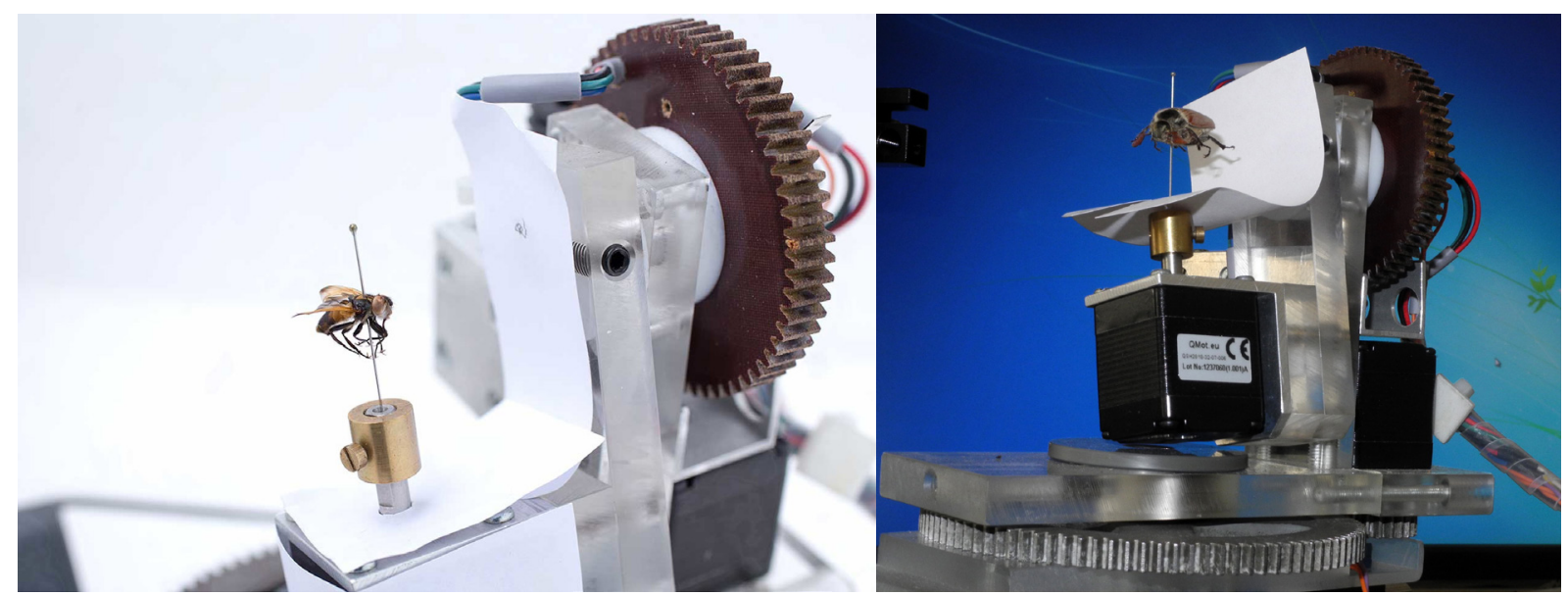

Fig. 2.17-2.18. Two different designs of the ZooSphere. In the first (left) figure, the stepper motor is centered on a long U-shaped axis, a swing, which is controlled by the second axis. In the second figure (right) all of this is more compressed in space and instead of a U-shaped axis to mount the first stepper motor, it is attached to the second by means of a T-shaped rod. Image copyright MfN.

\subsubsection{Collections}

The collections that are possible to digitise with the ZooSphere system are similar to those of the regular $2 \mathrm{D}+$ system; in essence small specimens, from $0.5-10 \mathrm{~cm}$, with the main focus on pinned insects. Of course, it is possible to picture larger specimens too, as long as they can be placed in the intersection of the two rotational axes. Unfortunately, it is not possible to picture specimens belonging to a wet collection without taking them out of the storage solution.

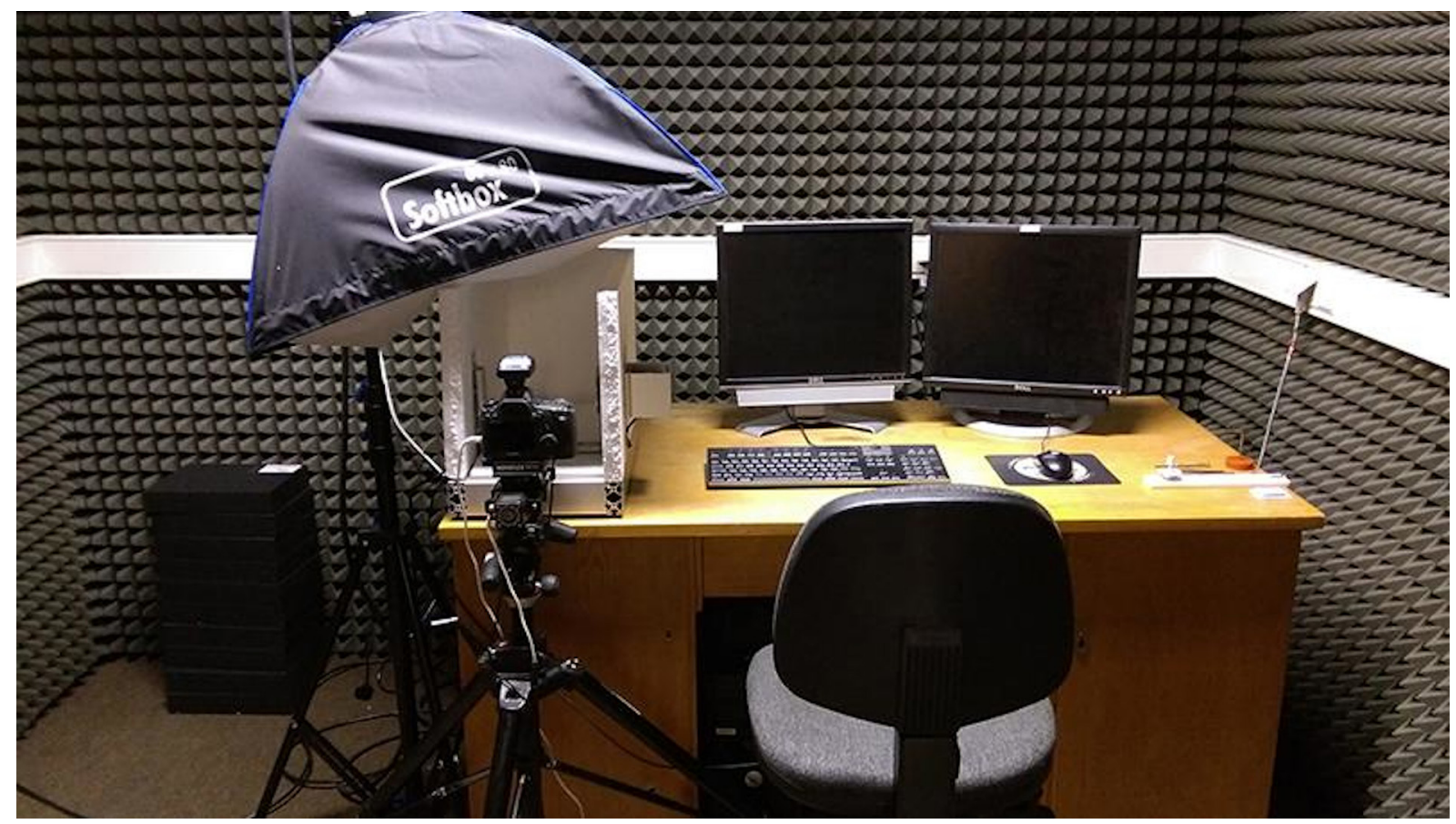

Fig. 2.19. View of the photographic lab of the ZooSphere, with the automated specimen rotator in front of the camera, covered by styrofoam panels to reflect the light in all directions. Image copyright MfN. 


\subsubsection{ZooSphere database}

Although the interface of the ZooSphere online database may change over time, the general information will stay the same. On the Zoosphere online database it is possible to browse through the specimens that are currently digitised (Fig. 2.20). Once a specimen is selected, a page with all the available metadata of the specimen together with the viewer for the focus stacked views (Fig. 2.21) will appear. The different planes cover the following: the contact information for the responsible party of the owner institution and the focus stacking data (Fig. 2.22); the viewer to browse through different views of the specimen (Fig. 2.23); the taxonomic tree of the specimen (Fig. 2.24); a picture of the labels of the specimen (Fig. 2.25) and a world distribution map of the species, as retrieved from GBIF (Fig. 2.26).

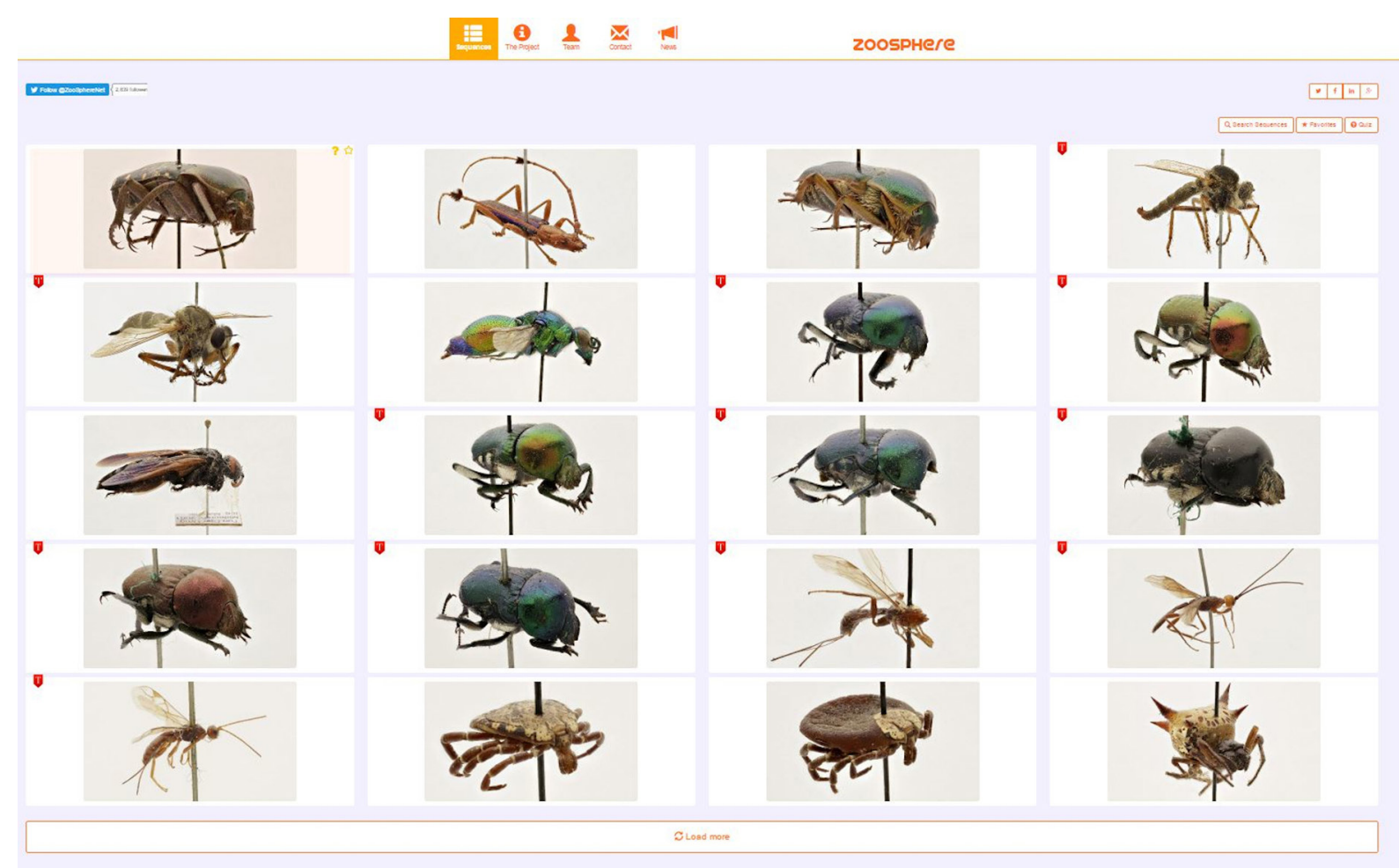

Fig. 2.20. Overview of the digitised specimen with the ZooSphere setup, visible at Zoosphere.net. Image copyright MfN.

Another system for automatic and multi-view 2D+ imaging, the Darmstadt Insect Scanner (DISC3D), has recently been published (for detailed information see Ströbel et al. 2018). Specimens (e.g., pinned insects) are mounted on a motorized two-axis gimbal (see fig. 2 in Ströbel et al. 2018), allowing imaging from almost any viewing angle (Ströbel et al. 2018: fig. 6). DISC3D continuously records images during camera movement of the macro-rail. Pre-calibrated 2D+ images are calculated online during the scanning process (Ströbel et al. 2018: fig. 4). In this way, the generated 2D+ images can provide depth information and fulfil the requirements of a pinhole camera model (single perspective, a substantial prerequisite for 3D modelling using SfM, see Chapter 1.2).

\subsubsection{References}

Ströbel B., Schmelzle S., Bluethgen N. \& Heethoff M. 2018. An automated device for the digitisation and 3D modelling of insects combining extended-depth-of-field and all-side multi-view imaging. Zookeys 759: 1-27. https://doi.org/10.3897/zookeys.759.24584

ZooSphere. 2018. Available from http://www.zoosphere.net/ [accessed 02 Jul. 2018]. 
BRECKO J. \& MATHYS A., Best practice handbook for 2D+/3D digitisation
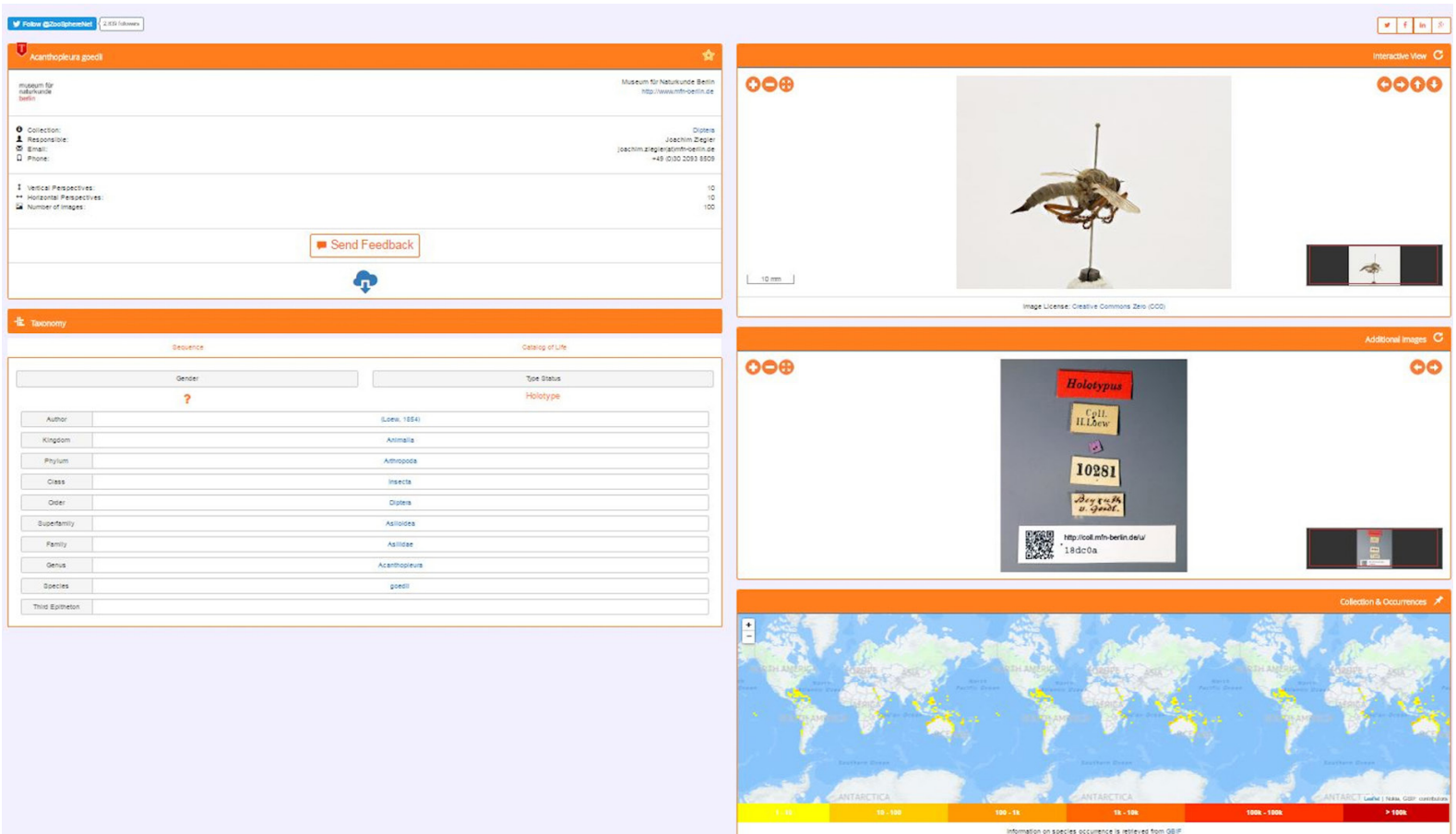

Fig. 2.21. Overview of the specimen page visible at Zoosphere.net. Image copyright MfN.

$\frac{\text { Send Feedback }}{10 \text { Se }}$

Fig. 2.22. Plane 1 of the specimen page at Zoosphere.net, showing the collections responsible and the acquisition metadata. Image copyright MfN. 


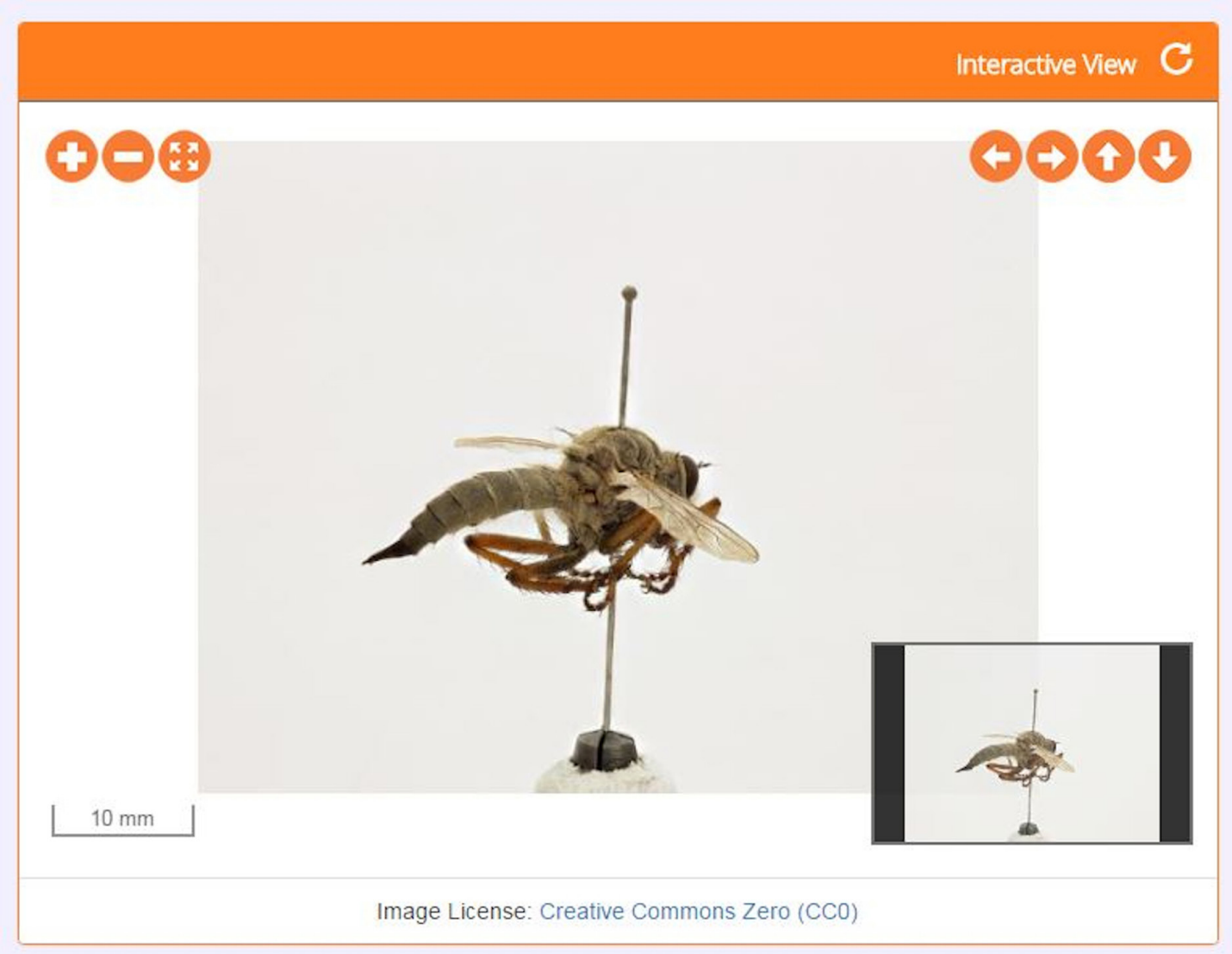

Fig. 2.23. Plane 2 of the specimen page at Zoosphere.net, showing the specimen pictures. Image copyright MfN.

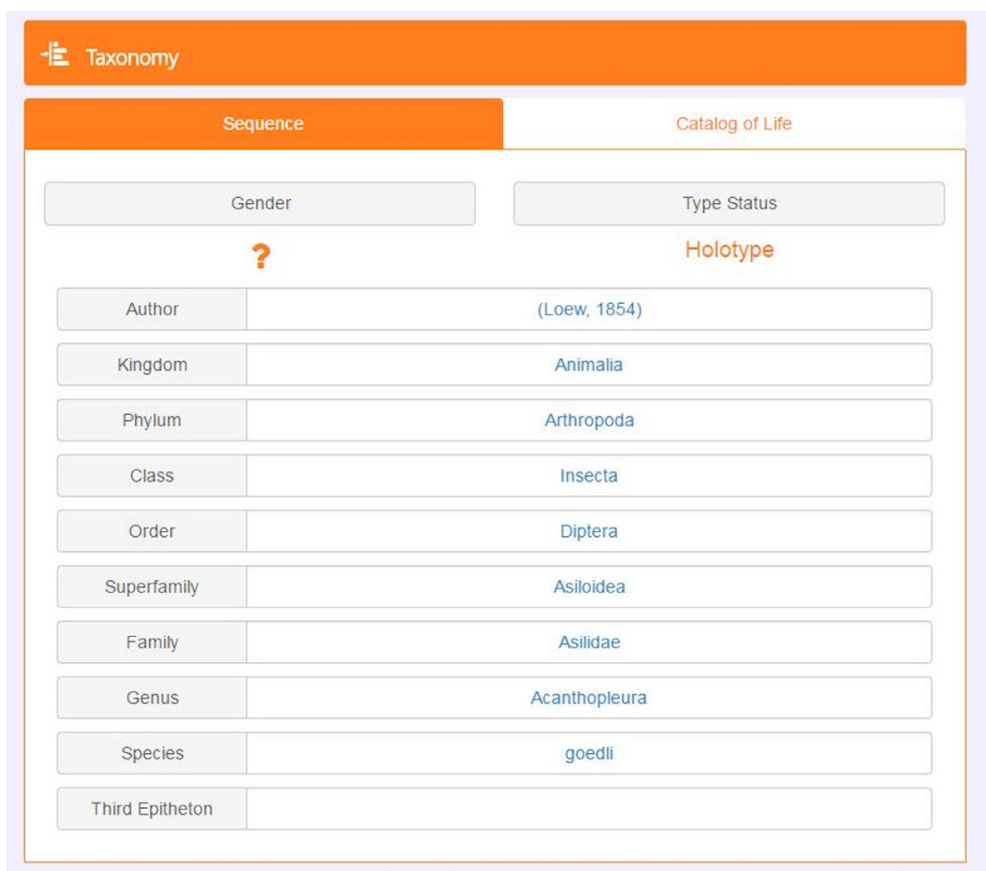

Fig. 2.24. Plane 3 of the specimen page at Zoosphere.net, showing the taxonomy of the specimen. Image copyright MfN. 
BRECKO J. \& MATHYS A., Best practice handbook for 2D+/3D digitisation

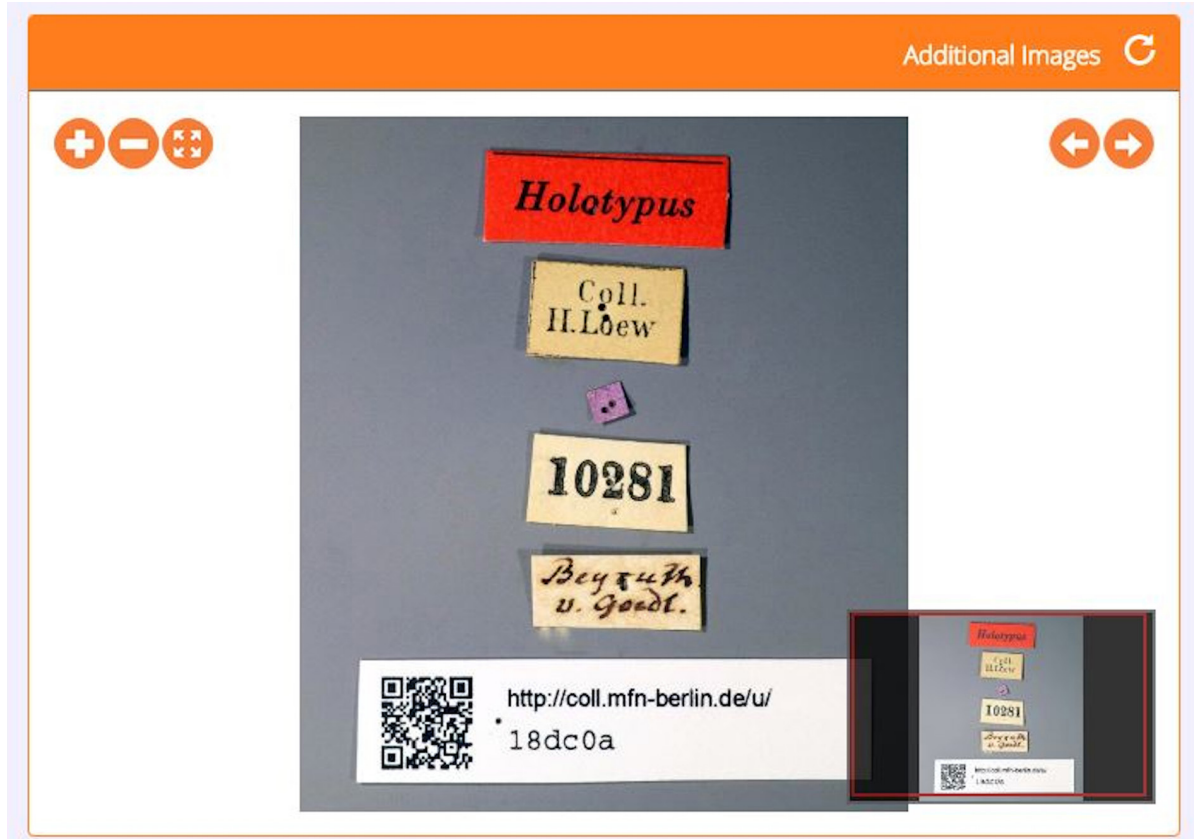

Fig. 2.25. Plane 4 of the specimen page at Zoosphere.net, showing the labels of the specimen. Image copyright MfN.

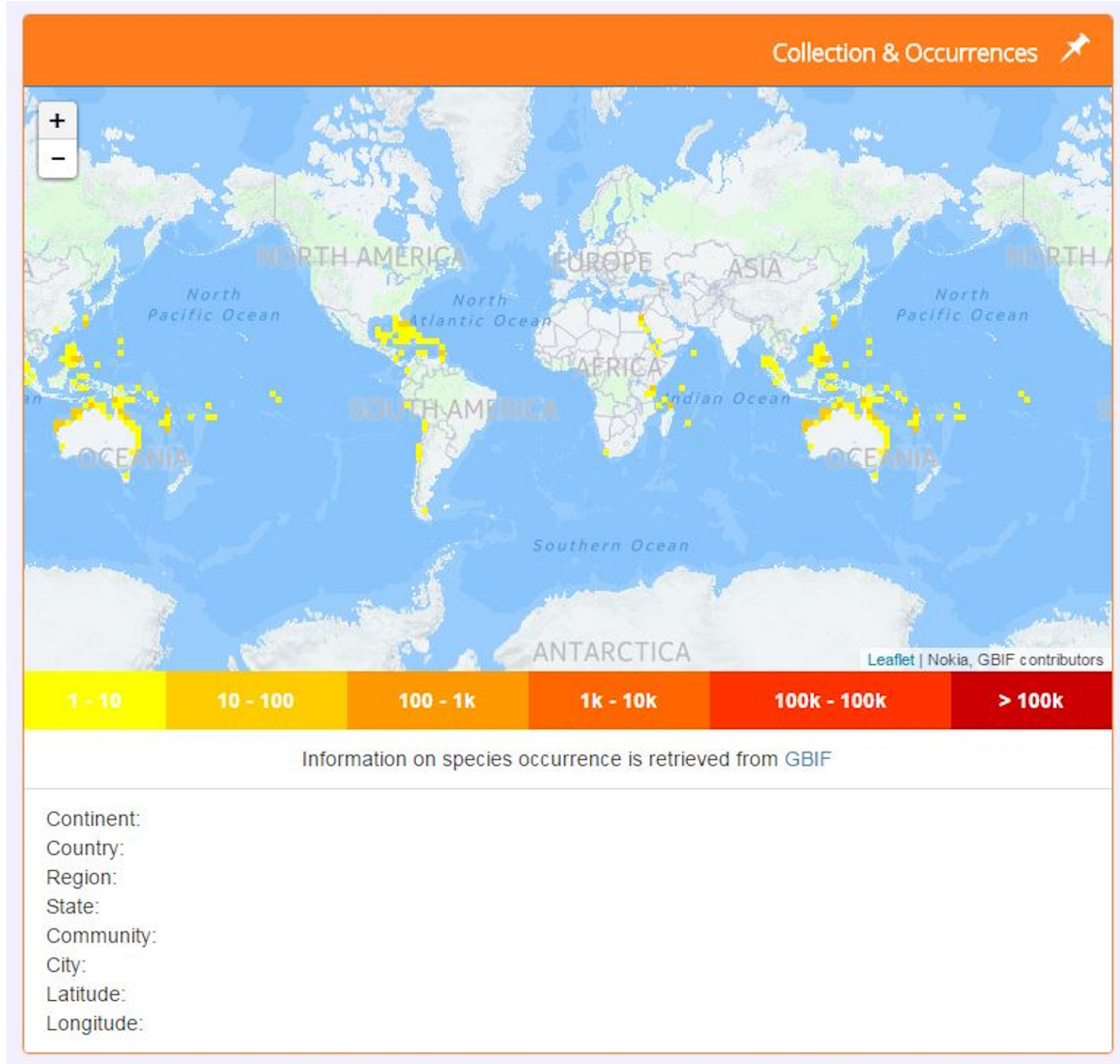

Fig. 2.26. Plane 5 of the specimen page at Zoosphere.net, showing the distribution of the specimen's species, as retrieved from GBIF. Image copyright MfN. 


\subsection{RTI and Portable Light Dome}

\subsubsection{Reflectance Transformation Imaging}

Reflectance Transformation Imaging (RTI) regroups a family of computational photographic methods that capture a subject's surface shape and colour (Fig. 2.27) and enable the interactive re-lighting of the subject from any direction. This is not $3 \mathrm{D}$, but it allows the enhancement of relief on the surface of the specimen.

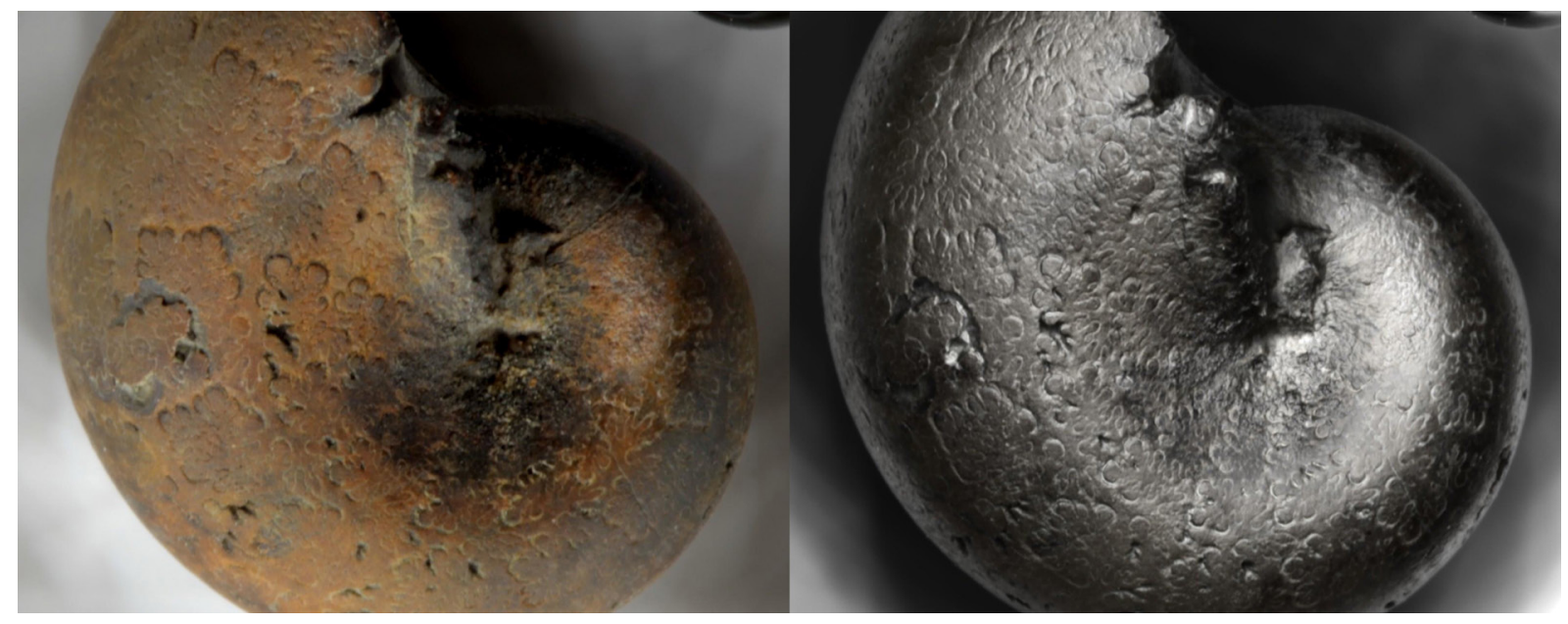

Fig. 2.27. Ammonite lighted with the RTI technique. Left normal view, right specular view.

There are two methods to capture RTI: the light array (dome) method and the highlight method (H-RTI).

The dome method consists of using a hemisphere (or similar setup) with a set of fixed lights with known positions. The camera is positioned at the top of the dome and the object to digitise is placed in the centre under the dome. The lights of the dome flash in a known sequence and a picture is taken for each light position.

The principle of the highlight method is similar, but the light position is unknown. Two reflective spheres (usually black or red) are placed on the scene next to the object and it is the reflection of the light on the sphere that will enable the software to recalculate the light positions (Fig. 2.28). The lights can be fixed or hand-held but have to be equidistant from the object captured. The advantage of the highlight method is that it allows to workwith different object sizes and locations. H-RTI was first used on Rock Art (Mudge et al. 2006). Details on this method can be found on the CHI website (http://culturalheritageimaging.org).

Different fitting algorithms can be applied to both capture methods. The most common ones are Polynomial Texture Mapping (PTM) and Hemispherical Harmonics (HSH). According to Manfredi et al. (2013, 2014), HSH is superior to PTM regarding "3D" quality because the normal extraction is more reproducible and therefore more sensitive to change. HSH also renders reflections while reflective objects in PTM usually appear matte and muted in texture. PTM, on the other hand, offers more rendering options.

\subsubsection{Material and methods}

The equipment needed to perform RTI (Table 2.3) consists of any type of camera that can mount a lens on it with minimal deformation, such as most fixed-focal lenses, whether it is a DSLR (full frame or APS-C) or mirrorless camera. 
Table 2.3. Equipment list to perform RTI

\begin{tabular}{c}
\hline Equipment \\
DSLR \\
DSLR remote shutter release \\
Macro Lens \\
Tripod \\
External Flash or Handheld Flashlight \\
2 Black/Red Spheres \\
Scale bar \\
Rope \\
RTIbuilder \\
RTIviewer (or any RTI viewer software) \\
\hline
\end{tabular}

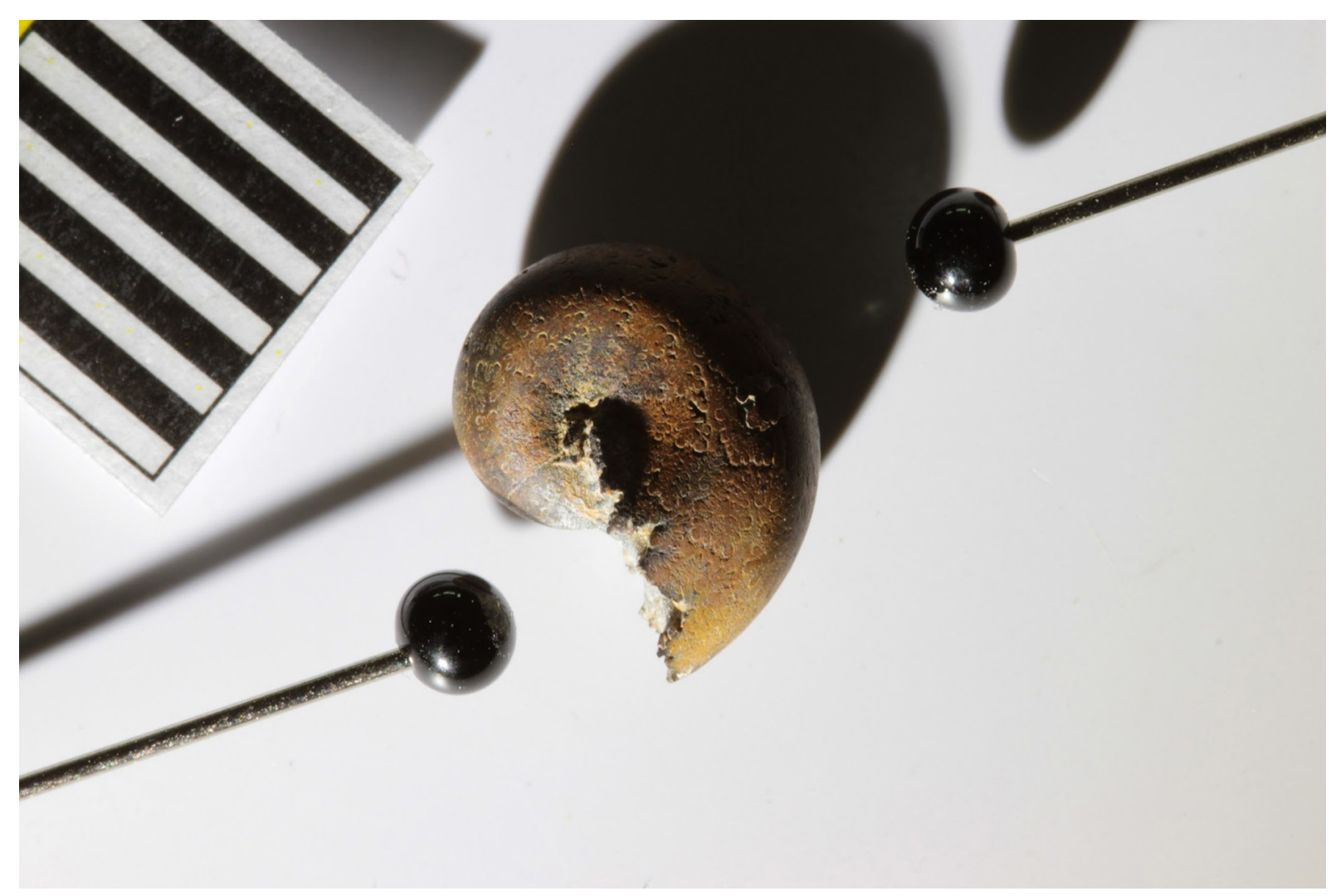

Fig. 2.28. Picture for H-RTI digitisation. The highlights on the black spheres allow the algorithm to reconstruct a RTI model. 
H-RTI was chosen with the method described on the CHI website (http://culturalheritageimaging.org). Images were processed with the RTIbuilder software (freeware available on the CHI website, Cultural Heritage Imaging 2018). Results are viewed with the RTIviewer (freeware available on the CHI website, Cultural Heritage Imaging 2018).

The RTI file is generated combining of several pictures taken from the same point of view with light coming from different orientations, equidistant to the object. The resulting RTI file calculates how light will reflect on the subject and records micro-relief on the surface from each orientation. It is a complementary technique to other digitisation techniques and can produce an astonishing level of surface detail. Videos with instructions on how to make a RTI recording and how to use the software can be found on the vimeo channel of CHI (https://vimeo.com/culturalheritage).

Tests with several lights (LED flash-light, LED $30 \mathrm{~W}$, LED $24 \mathrm{~W}$, cold light) of different intensities and angles gave quite similar results. However, it is best that no other light should influence the pictures taken. The best results were obtained when at least 3-4 different angles are chosen with 12-16 images per $360^{\circ}$. This results in a minimum set of 36 and a maximum set of 64 pictures.

\subsubsection{Collections}

Any collection harbouring specimens that have micro details or structures that are difficult to visualise using a regular light setup can benefit from digitisation using the H-RTI method. Details that are not clearly visible due to an interfering colour, pattern or both are easily revealed with this technique. The RTI technique, although originated in the cultural heritage field, is often applied to specimens of the following collections: geology, palaeontology and anthropology/prehistory as these collections harbour many specimens with the above criteria. However, specimens with these criteria can be found in the entomology and recent (in)vertebrate collections, although it has not been tested to a similar degree as the other collections.

\subsubsection{MiniDome/Portable Light Dome}

The Portable Light Dome (PLD or Minidome) is a portable automated device of photometric stereo (Minidome, SD; Portable Light Dome, 2017). It was developed by VISICS at the Katholieke Universiteit Leuven in Belgium, in order to digitise cuneiform tablets (Willems et al. 2005; Faculteit Letteren KULeuven, 2016). It is inspired by the early works of Tom Malzbender (2001) and the dome approach for RTI (Hameeuw 2014). The PLD consists of a hemisphere with 260 LED lights with known positions (Fig. 2.29). Instead of using the Malzbender PTM algorithm, the PLD uses Photometric Stereo (PS). Like RTI, PS uses multiple images from the same viewpoints but different illumination points. PS enables the calculaion of the normals of a surface and the albedo (Salvant et al. 2017). For those calculations, it uses the intensity of the reflected light and the angle of incidence of the light on the object (MacDonald 2014). The results allow for photorealistic virtual relighting and a 3D surface of the object (only for the view captured).

\subsubsection{Material and methods}

A colour Allied Vision Prosilica GX 6600 camera of $28.8 \mathrm{Mpx}$ is mounted on the Portable Light Dome (Fig 2.29). The time needed for the acquisition and the treatment is about 6 minutes per surface (it can be reduced by using a camera with less resolution). In that time, a picture is taken each time a different LED (260 in total) is illuminated, creating a similar, yet more accurate result as the manual RTI previously discussed. As mentioned previously, results include a 3D reconstruction of the object for the captured view. However, there is some deformation near the borders of the reconstructed 3D model. A nice feature of this software is that it is able to automatically produce artistic renderings using specific filters (Figs 2.30-2.31). This unique feature can be very important in the publication workflow of new specimens/species. Nevertheless, the PLD is still a prototype and is not yet available on the market. 


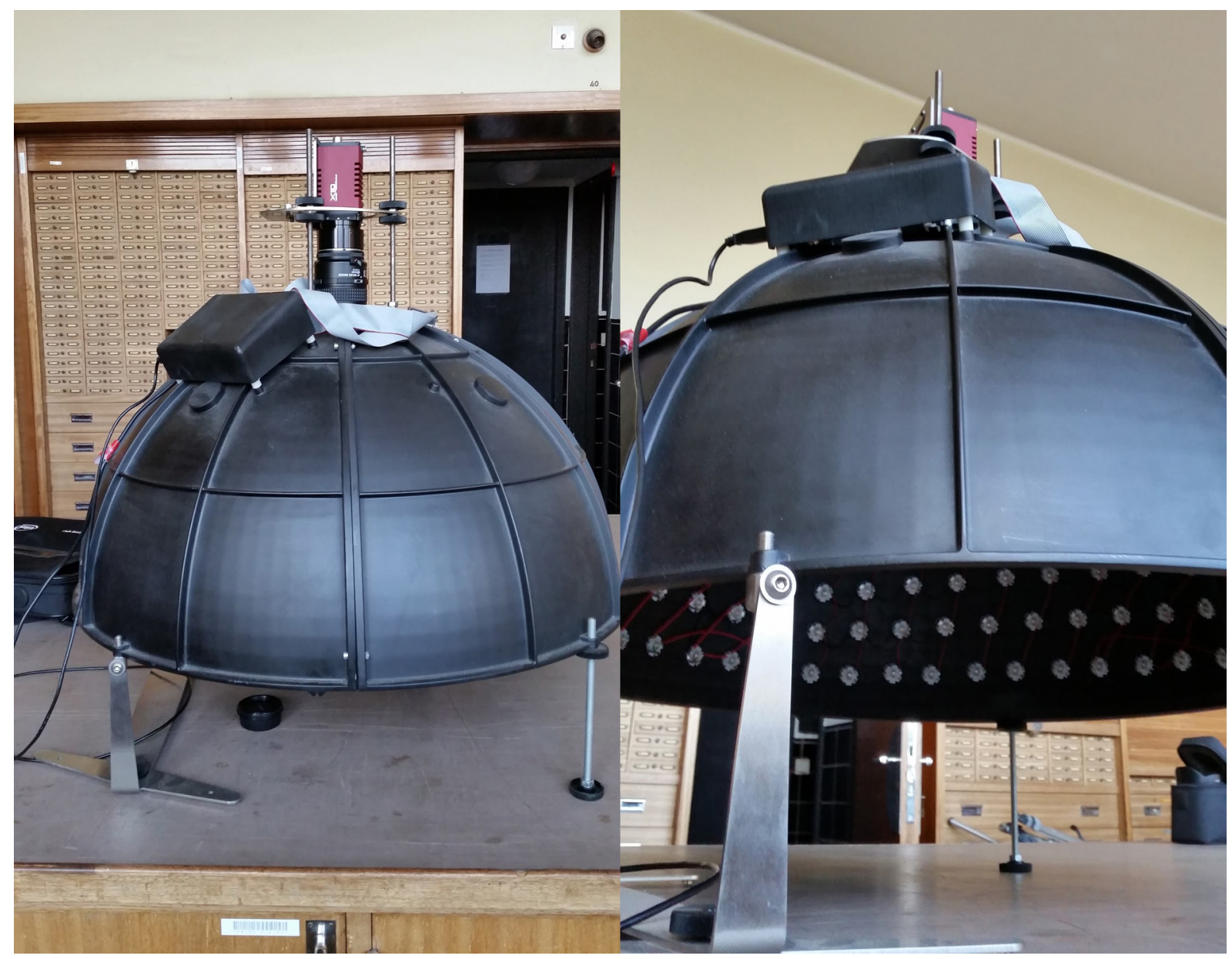

Fig. 2.29. The PLD setup. Left: general view of the dome with the machine vision camera mounted on top. Right: view of some of the 260 LEDs of the dome.

This limits the potential of this technology which, at the moment, is not yet a solution for a massive digitisation program of European natural history collections.

A multiband version of the PLD exists following the RICH (Watteeuw et al. 2017) and EES project (Van der Perre et al. 2016). Instead of white LED lights it uses UV (365 nm), blue (465 nm), green $(523 \mathrm{~nm})$, red $(623 \mathrm{~nm})$ and IR $(850 \mathrm{~nm})$.

\subsubsection{Collections}

Any type of specimen can be completely or partially (depending on the size) digitised with the PLD. The most interesting collections would be those with fine details that are very difficult to capture with regular photography and regular light conditions, especially specimens that show different details when turned in different directions in a light source; these are often found in geology, palaeontology and anthropology/ prehistory collections. The technique can also be used to investigate the colour and reflectance of a specimen's surface by observing change depending on the light position. Specimens from entomology (vertebrates or invertebrates) can exhibit a change in colour depending on the lighting position as many have anisotropic surfaces, making them good candidates for colour gradient capture using the PLD.

\subsubsection{Other RTI domes}

Many other RTI domes like the PLD exist (Earl et al. 2011; Avgousti et al. 2017; MacDonald 2011; Padfield \& Saunders 2005; Piquette 2011). All differ in hardware configuration; some use the RTI-CHI 
software, others have built their own algorithms. It is even possible to build a dome from scratch by following one of the many tutorials available online (Kinsman 2016; Pawlowicz 2016).

\subsubsection{References}

Avgousti A., Nikolaidou A. \& Georgiou R. 2017. Openumisma: a software platform managing numismatic collections with a particular focus on reflectance transformation imaging. Code for Libraries Journal 37: 1-14.

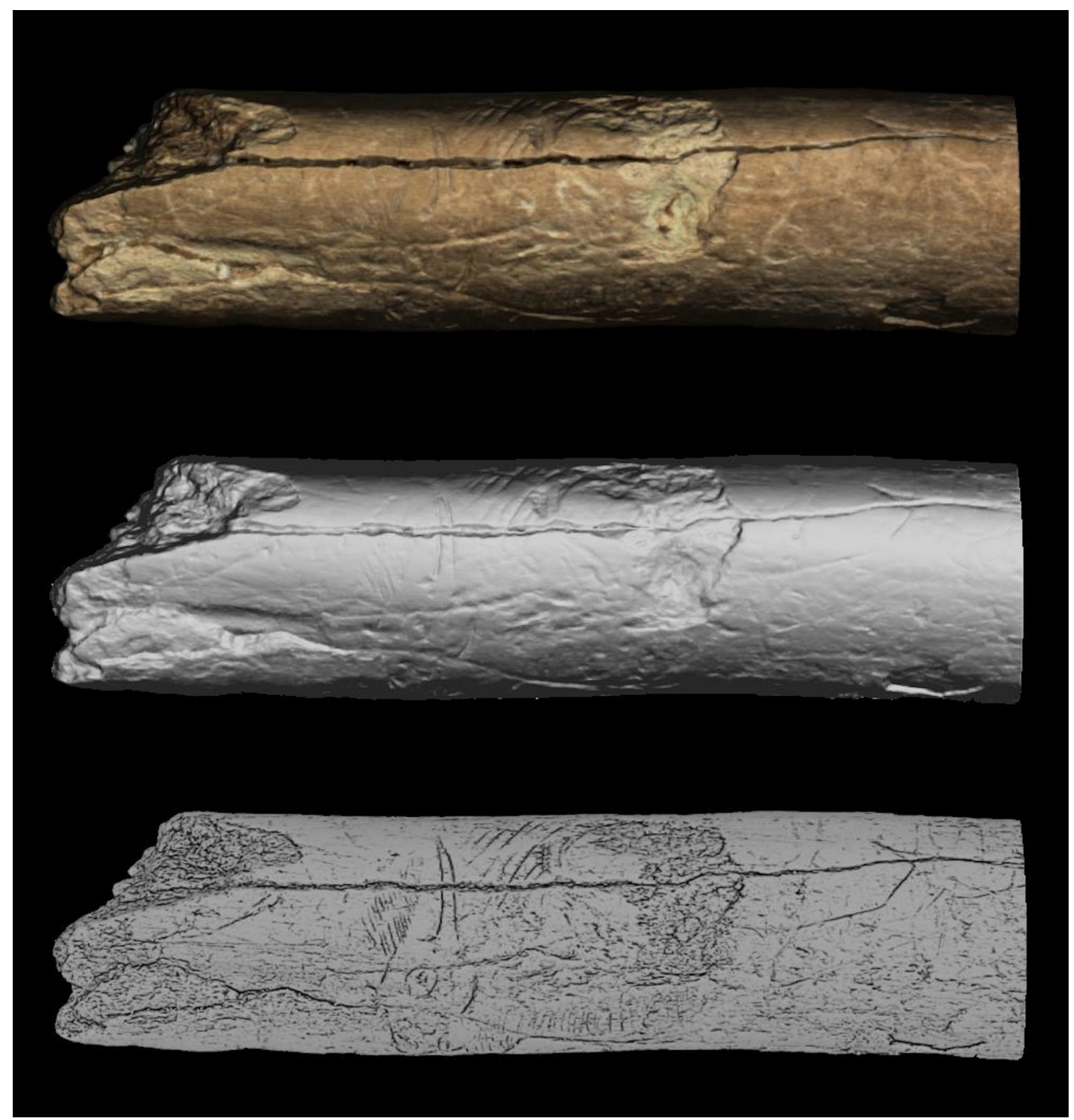

Fig. 2.30. 3D model of a mobile Palaeolithic art from "le Trou des Nutons". Above: coloured surface model; middle: Surface without texture; below: image processed with automatic filtering method to highlight surface features of bison bone. The acquisition was made with a 5 Mpx RGB machine vision camera. 
Cultural Heritage Imaging. 2018. Available from http://culturalheritageimaging.org/ [accessed 24 Jul. 2018].

Earl G., Basford P.J., Bischoff A.S., Bowman A., Crowther C., Dahl J., Hodgson M., Martinez K., Isaksen L., Pagi H., Piquette K.E. \& Kotoula E. 2011. Reflectance transformation imaging systems for ancient documentary artefacts. In: Bowen J.P., Dunn, Stuart \& Ng K. (eds) Proceedings of the 2011 International Conference on Electronic Visualisation and the Arts (EVA'11): 147-154. BCS Learning \& Development Ltd., Swindon.

Faculteit Letteren - KULeuven. 2016. Leuven Cuneiform Collection. Available from http://www.arts.kuleuven.be/ono/meso/projects/cuneiformcollection [accessed 23 Jul. 2018].

Hameeuw H. 2014. Portable Light Dome System: from Registration to Online Publication within the Hour. Status Quaestionis Portable Light Dome Project for Cuneiform Documents. KMKG-MRAH, Leuven. Available from https://portablelightdome.files.wordpress.com/2014/12/portable-light-domesystem-from-registration-to-online-publication-within-the-hour_1-1.pdf [accessed 29 Aug. 2018].

Kinsman T. 2016. Using Reflectance Transformation Imaging to Shoot Ultra-Detailed Macro. Available from https:/petapixel.com/2016/04/21/shoot-super-detailed-macro-photographs-rti-camera-rig/ [accessed 25 Jul. 2018].

MacDonald L.W. 2011. Visualising an egyptian artefact in 3D: comparing RTI with laser scanning. In: Bowen J.P., Dunn, Stuart \& Ng K. (eds) Proceedings of the 2011 International Conference on Electronic Visualisation and the Arts (EVA'11): 155-162. BCS Learning \& Development Ltd., Swindon.

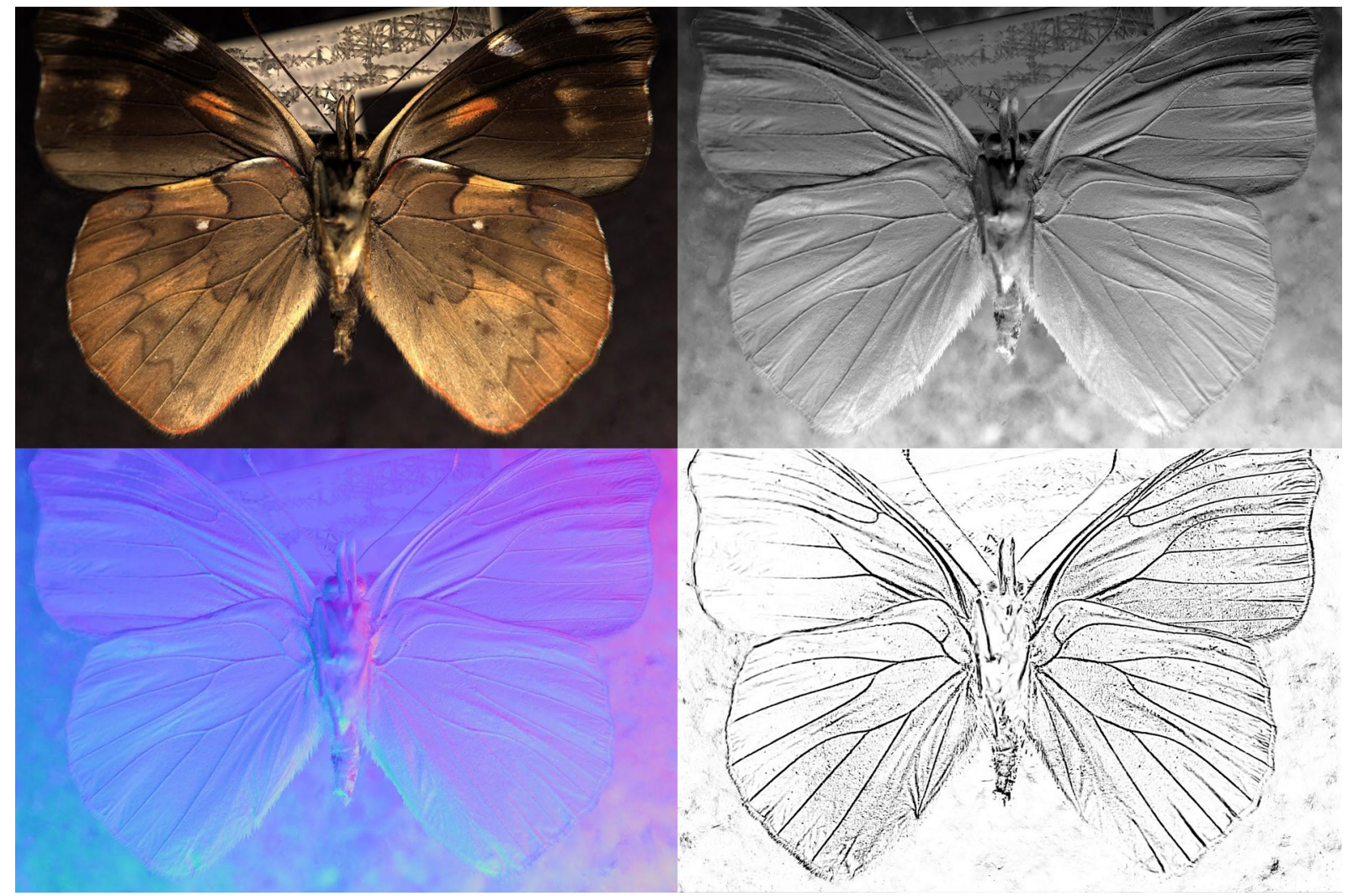

Fig. 2.31. Perisama cardases captured with PLD. The normal colour image with relighting option is found on the upper left. Upper right is the grey scale image, bottom left the normal map and bottom right algorithmically-generated sketch using a filter. 
MacDonald L.W. 2014. Colour and directionality in surface reflectance. 40th Annual Convention of the Society for the Study of Artificial Intelligence and the Simulation of Behaviour (AISB 2014): 223-229.

Malzbender T., Gelb D. \& Wolters H.J. 2001. Polynomial texture maps. Proceedings of the $28^{\text {th }}$ Annual Conference on Computer Graphics and Interactive Techniques (SIGGRAPH 2001): 519-528. ACM, Los Angeles.

Manfredi M., Williamson G., Kronkright D., Doehne E., Jacobs M., Marengo E. \& Bearman G. 2013. Measuring Changes in Cultural Heritage Objects with Reflectance Transformation Imaging. In: Addison A.C., De Luca L., Guidi G., Pescarin S. (eds) 2013 Digital Heritage International Congress (DigitalHeritage): 189-192. IEEE, Marseilles. https://doi.org/10.1109/DigitalHeritage.2013.6743730

Manfredi M., Bearman G. H., Williamson G., Kronkright D., Doehne E., Jacobs M. \& Marengo E. 2014. A new quantitative method for the non-invasive documentation of morphological damage in paintings using RTI surface normals. Sensors 14 (7): 12271-12284. https://doi.org/10.3390/s140712271

Minidome. SD. Minidome Webpage. Available from http://www.minidome.be/v01/home.php [accessed 23 Jul. 2018].

Mudge M., Malzbender T., Schroer C. \& Lum, M. 2006. New Reflection Transformation imaging methods for rock art and multiple-viewpoint display. The $7^{\text {th }}$ International Symposium on Virtual Reality, Archaeology and Cultural Heritage (VAST2006): 195-200.

Padfield J. \& Saunders D. 2005. Polynomial texture mapping: a new tool for examining the surface of paintings. ICOM-CC Preprints, the Hague (1): 504-510.

Pawlowicz L. 2016. Affordable Reflectance Transformation Imaging Dome. Available from https:/hackaday.io/project/11951-affordable-reflectance-transformation-imaging-dome [accessed 25 Jul. 2018].

Piquette K. 2011. Reflectance transformation imaging (RTI) and ancient egyptian material culture. Damqatum: The CEHAO Newsletter - El boletin de Noticias del CEHAO 7: 16-22.

Portable Light Dome. 2017. Portable Light Dome, Relighting Cultural Heritage. Available from https://portablelightdome.wordpress.com/ [accessed 23 Jul. 2018].

Salvant J., Walton M., Kronkright D., Yeh C-K., Li F., Cossairt O. \& Katsaggelo A.K. 2017. Photometric Stereo by UV-Induced Fluorescence to Detect Protrusions on Georgia O'Keeffe's Paintings. Computing Research Repository Graphics (cs.GR) abs/1711.08103. https://arxiv.org/abs/1711.08103

Van der Perre A., Hameeuw H., Boschloos V., Delvaux L. Proesmans M., Vandermeulen B., Van Gool L. \& Watteeuw L. 2016. Towards a combined use of IR, UV and 3D-imaging for the study of small inscribed and illuminated artefacts. In: Homom P.M. (ed.) Lights On ... Cultural Heritage and Museums!: 163192. FLUP, University of Porto, Porto.

Watteeuw L., Vandermeulen B., Van der Stock J., Delsaerdt P., Gradmann S. \& Truyen F. 2017. Illuminare, Centre for study of Medieval Art. RICH Project. Available from http://ec2-34-244-170-214.eu-west-1.compute.amazonaws.com/accordion-item/rich/ [accessed $23 \mathrm{Jul}$. 2018].

Willems G., Verbiest F., Moreau W., Hameeuw H., Van Lerberghe K. \& Van Gool L. 2005. Easy and cost-effective cuneiform digitising. The $6^{\text {th }}$ International Symposium on Virtual Reality, Archaeology and Cultural Heritage VAST. http://hdl.handle.net/1854/LU-8572126 


\section{3D digitisation}

\subsection{Structure from Motion (SfM)}

Structure from motion (SfM), a type of photogrammetry when both the camera and object position are unknown, is a technique which computationally builds point clouds of an object or a site based on pictures of it. 3D models are generated using an algorithm by meshing the point clouds. The requisite 2D images need to be taken from different angles and positions, with sufficient overlap between the images to allow the identification of matching points. SfM is a great tool to 'scan' a specimen and compute a 3D model of it afterwards. The technique itself is quite fast, inexpensive and open to anyone with a camera and the basic knowledge of how to use it. There are several ways of taking pictures to construct a 3D model (Luhmann et al 2016). But there are some guidelines that need to be followed to be successful time after time.

Different software is available and most allow the user to generate surface meshes and to export models in various formats. The software used at RBINS, RMCA and by DISC3D is Agisoft Metashape (Professional) (previously Agisoft Photoscan). The reasons for this choice are the accuracy, the platform which is not cloud-based, the low price for educational institutes, the ability to scale a model and its ease of use (Mallison \&Wings 2014).

It is possible to calculate photorealistic textures, using the SfM models which make them great for visualisation purposes. Several studies have attested to the accuracy of the 3D model obtained with this technique (Evin et al. 2016; Giacomini et al. 2017b). Another advantage of SfM is the possibility of recording objects of any size (from a few centimeters to several kilometers).

Accuracy is variable depending on the equipment (camera resolution, lens quality, tripod, lights, etc.), the acquisition parameters (focal, ISO, exposure), protocol (number of pictures, orientations) and the processing parameters; but in good conditions, it is possible to record an object with details of approximately $50 \mu \mathrm{m}$ (Guery et al. 2017).

Because of the reliable accuracy once the same protocol is used for each acquisition (Evin et al. 2016; Giacomini et al. 2017a), it is possible to use SfM models as a digital twin to send to researchers that are unable to consult the physical collection, or if a specimen is too big to leave a collection/excavation site (Remondino et al. 2010; Remondino 2011; Historic England 2017; Falkingham et al. 2018; Kreij et al. 2018). The 3D models made by SfM are already used in geometric morphometrics (Remondino et al. 2010; Lallensack et al. 2016; Muñoz-Muñoz et al. 2016; Marcy et al. 2018), restoration of specimens (Lautenschlager 2016), and in ecomorphological studies (Giacomini et al. 2017b; Beltran et al. 2018).

\subsubsection{SfM of large specimens $(>2 \mathrm{~cm})$}

\subsubsection{Material and methods}

The equipment needed to perform SfM (Table 3.1) consists of any type of camera on which a good lens can be mounted, whether it is DSLR (full frame or APS-C) or mirrorless. Using a macro lens with a fixed focal length allows for better models than using zoom lenses.

SfM models can also be obtained with lower consumer-grade cameras or smartphone cameras but results show lower accuracy (Barbero-García et al. 2017; Hernandez \& Lemaire 2016; Micheletti et al. 2014; Micheletti et al. 2015), therefore it is not recommended to use these within wide digitisation schemes.

The use of a light tent (Fig. 3.1) or softbox is advised in order to obtain homogeneous lighting, avoiding shadows and reducing reflections. 
Table 3.1. Equipment list to perform photogrammetry of large specimens

\begin{tabular}{c}
\hline Equipment \\
\hline DSLR or mirrorless camera \\
Automatic turntable + AGORA 3D software \\
Macro lenses \\
Tripod \\
Light setup or light tent \\
Different uniform materials for background (white, grey, black) \\
Supports or plasticine to hold the specimen in position \\
Scale bars \& photogrammetric markers \\
Colour checker \\
Agisoft Photoscan (Professional)
\end{tabular}

It is possible to generate a 3D model out of low-quality pictures or random shots of an object. However, this way of capturing a specimen is likely to produce poor 3D models. At best, they will appear acceptable when displayed with texture (colour), but will have a low amount of triangles, resulting in a bad mesh. The basis of an SfM model is formed by pictures of the object: if the pictures are good, the resulting 3D model will render the quality; if they are poor, the 3D model will reflect this.

If you keep the following principles in mind, a quality 3D model is only clicks away.

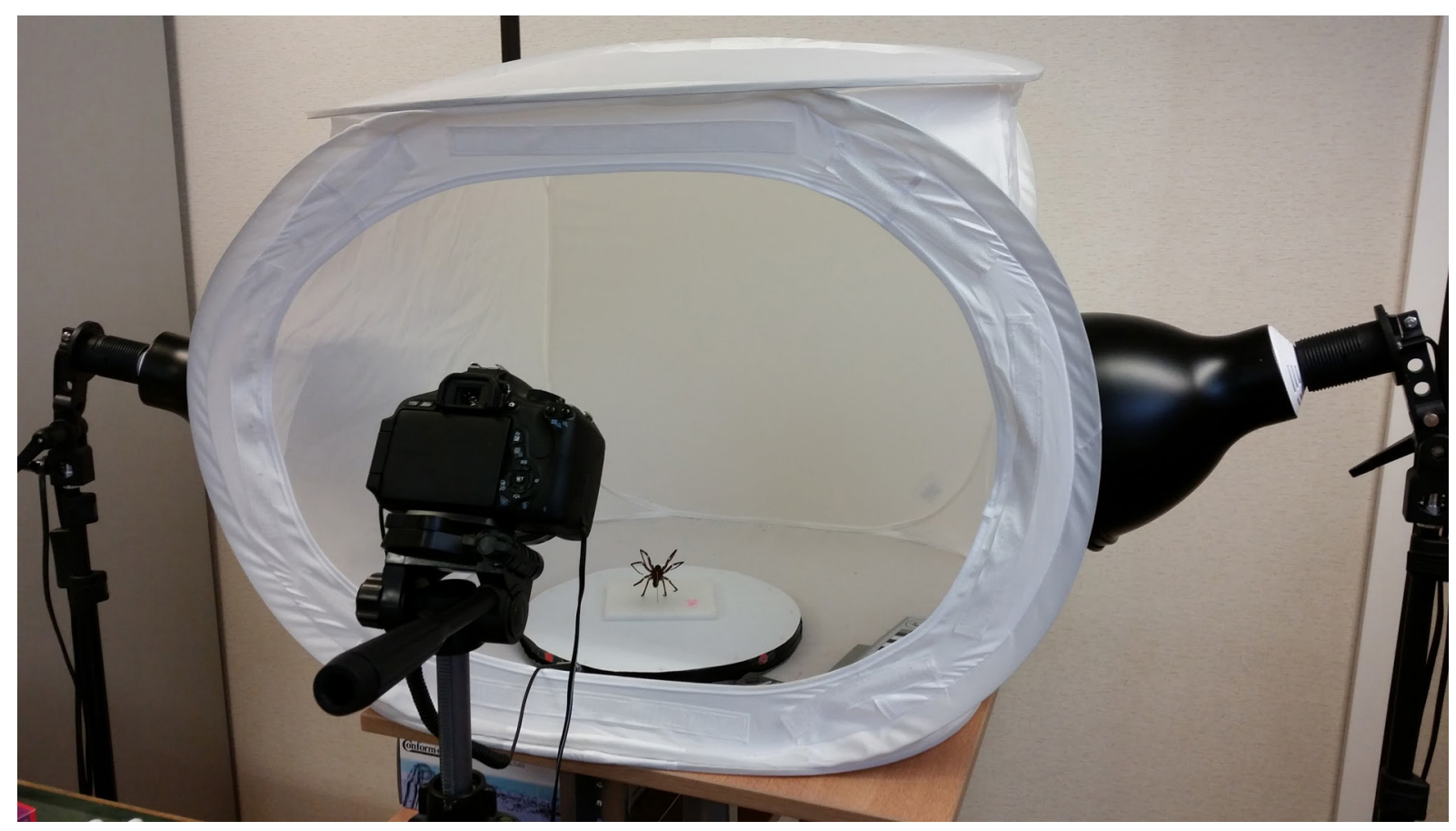

Fig. 3.1. Photogrammetry set-up with a specimen on a manual turntable, a Canon DSLR on a tripod, 2 studio lights and a light tent. 
In order to obtain a good 3D model from photographs, the pictures need to be captured from different positions and not from different angles of a single camera position (Fig. 3.2). This means that every picture, in essence, is a different camera position. It is important to avoid taking pictures from the same position relative to the object. Rather, try to circle around the object or keep the camera fixed and let the object rotate around its axis using a rotary table. It is best to capture the object from several rotations, rather than a single rotation, to avoid deformations.

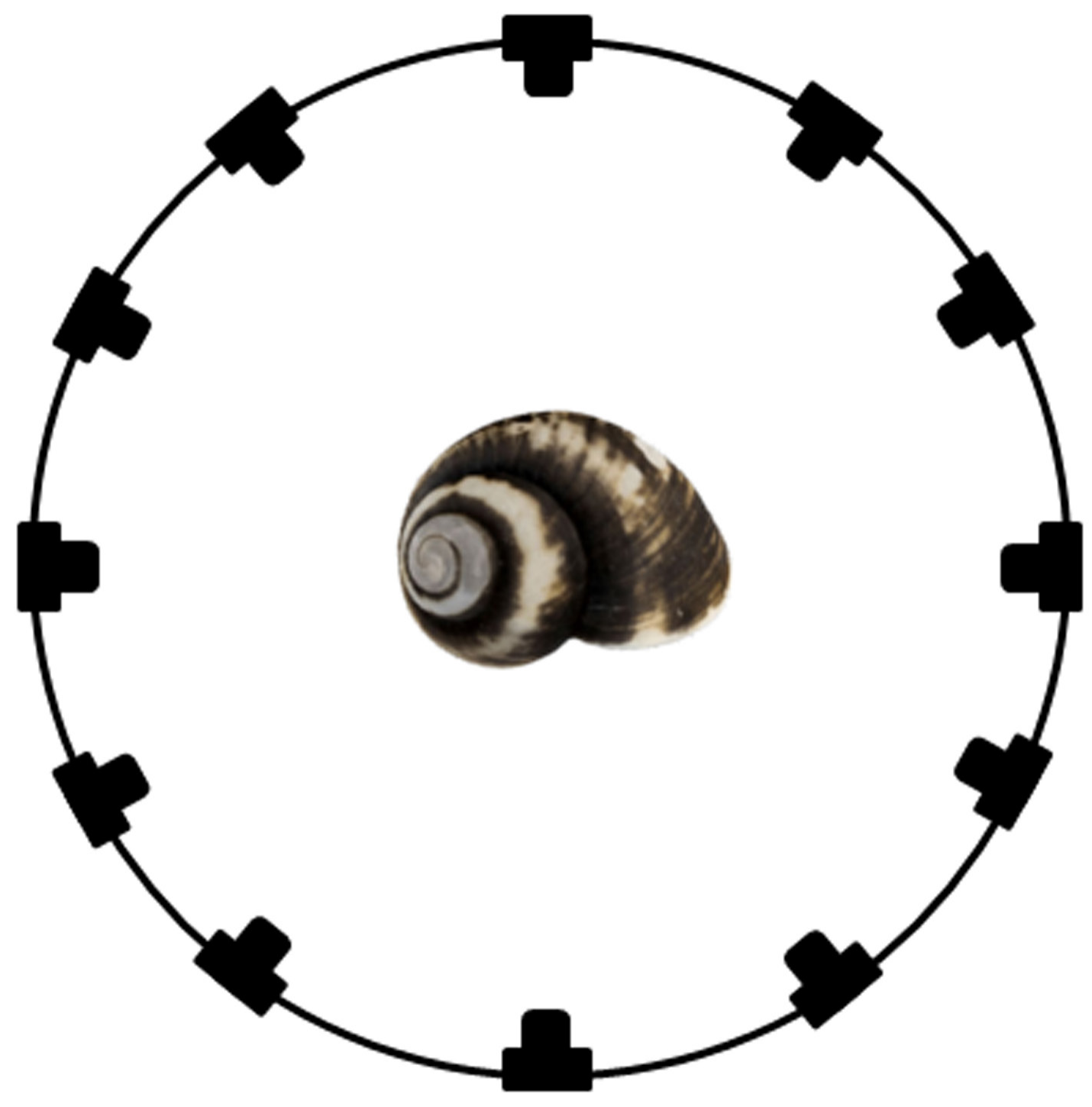

Fig. 3.2. Camera positions of a single rotation when taking pictures of an object.

When using a rotary table, if the specimen is not too fragile and if the parts do not move when turned on another side, it is best to place the object on at least three different sides, so practically all parts are viewed from different angles (Fig. 3.3). Taking pictures in this organised manner results in high quality 3D models. Sometimes it might be necessary to make additional pictures of some key positions to capture everything. For instance, when one part of the specimen is covered by another part (like the underside of a statue's arm, cavities of a skull, etc.), it is necessary to take a few pictures of the covered part from different angles to make sure the information is not missing in the 3D model. Remember, if it is not visible on several pictures, it is not captured in the 3D model. More tips and tricks on dealing with 


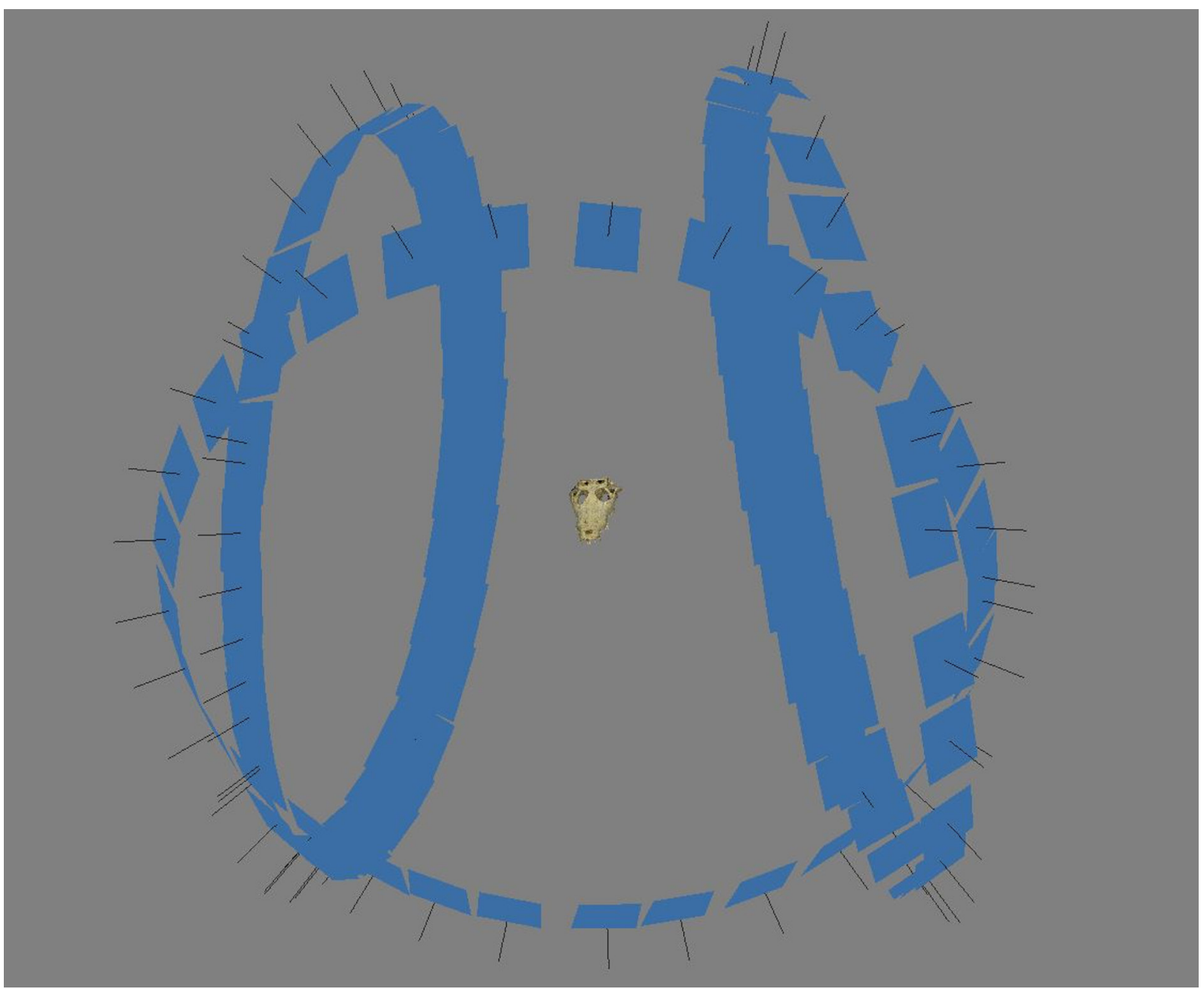

Fig. 3.3. Camera positions of three rotations. The top part of the crocodile skull was placed on a rotary table and turned on three different sides after a full turn. During the photoshoot the camera was kept at a fixed position.

difficult objects (translucent, bright, shiny, dark, etc.) can be found in Chapter 6, part 6.4: Challenging materials (page 94).

\subsubsection{AGORA 3D Automated Photographer}

At the start of the digitisation project at RBINS and RMCA, SfM was done by manually rotating the specimen using a rotary table. Fortunately, the AGORA3D Automated Photographer designed by Yvan Boumal of RBINS is now used. It allows the user to select how many pictures the camera needs to take for one rotation (Figs 3.4-3.5). After a rotation is finished a sound alerts the user to change the position of the object and start a new sequence. It is also possible to change basic camera settings like shutter speed, aperture, ISO value, image quality, AE mode, metering mode and exposure compensation. Once the camera and the relays are found, and the preferred settings are chosen, a directory to save the images is selected. In case of problems, the software has an emergency stop to abort all actions. It is possible to connect multiple lights to the controller which can be switched on or off within the software. The connection to the computer is with a serial port, which can be found on older power stations. Tests with converters on a USB or using ports on a laptop proved to be unsuccessful due to the lack of sufficient power. The major strong point is the reduction of human interaction time. Doing SfM in this way enables the operator to spend more time selecting or preparing specimens and afterwards 

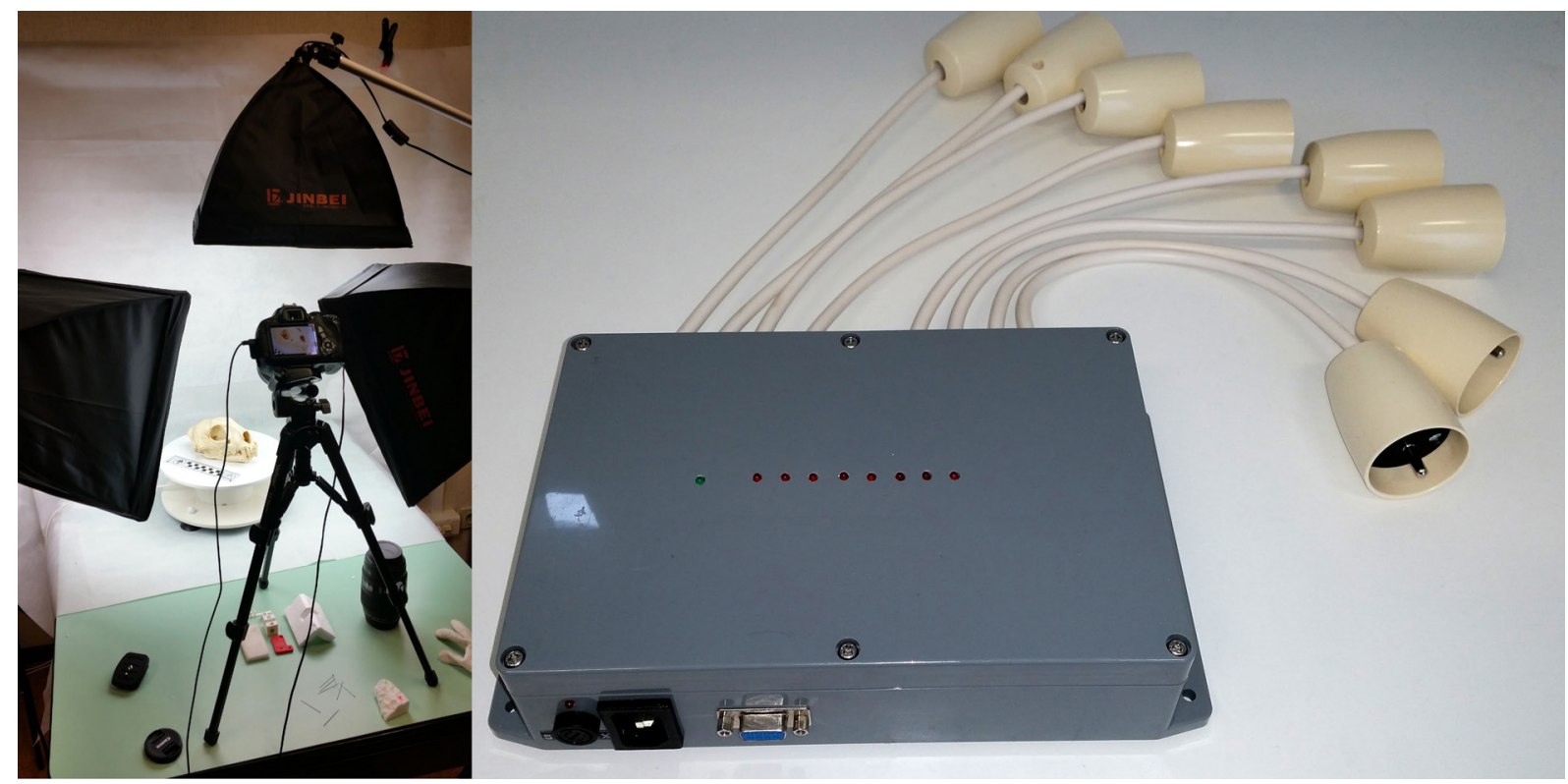

Fig. 3.4. The photogrammetry setup on the left with the automated turntable. The controller enables to command both a turntable and a camera through the AGORA 3D interface.

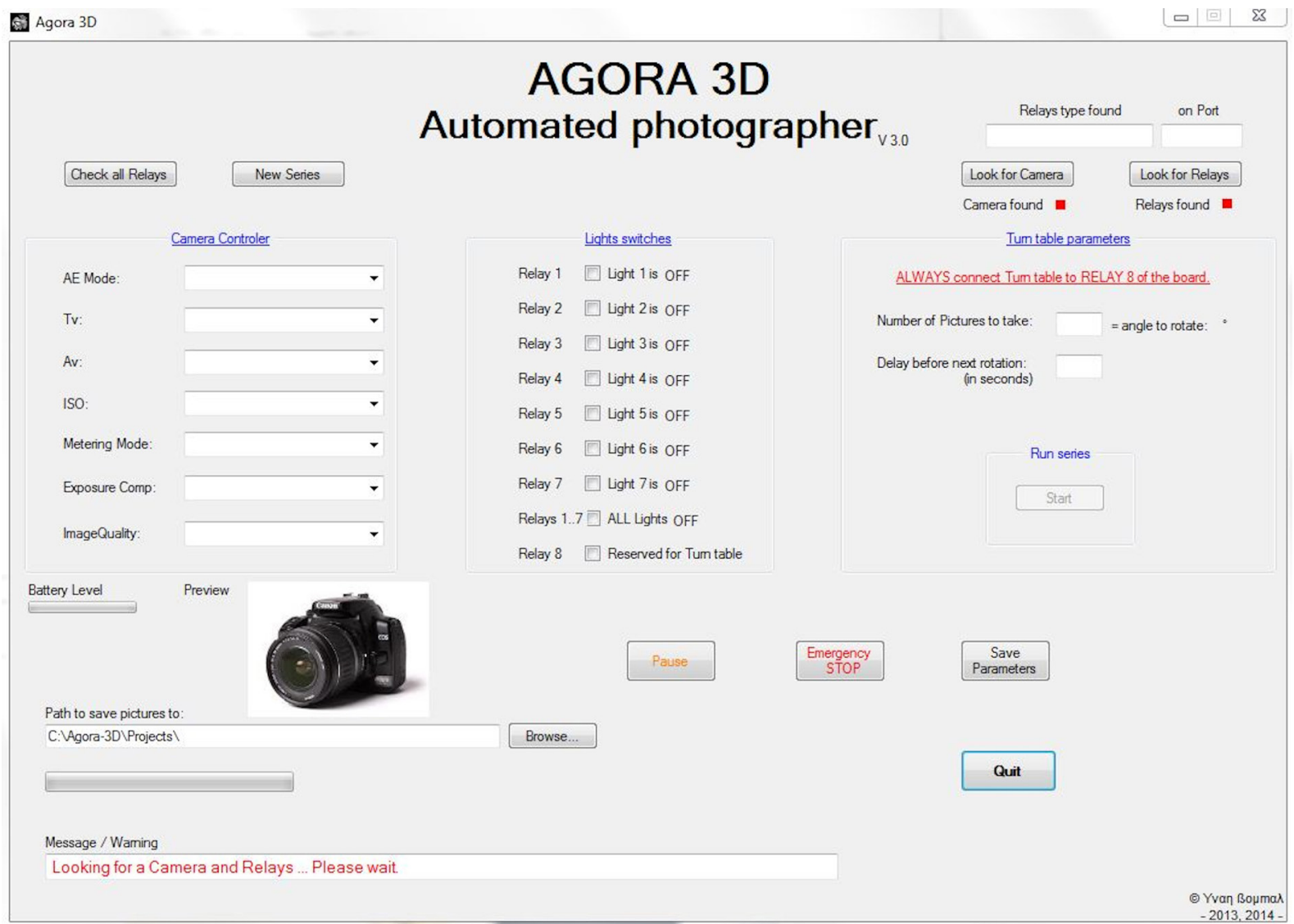

Fig. 3.5. Interface of the AGORA 3D Automated Photographer. 
dealing with the SfM software to generate the 3D models. The system could be improved to run on new devices like tablets and smartphones, making it more portable like the recent PINE controller (https://blackforestmotion.com/controller/) or the Foldio360 (http://orangemonkie.com/foldio360/).

\subsubsection{Collections}

During the several years of digitisation efforts at RMCA and RBINS, numerous specimens have been digitised using SfM. Almost any collection can be digitised using SfM as it is very versatile. If it can be pictured and if the details desired for the 3D model are visible in the 2D image, it can be processed using SfM. For some specimens, a slightly different approach or more advanced knowledge of the technique is required, but these cases are addressed in Chapter 6: Comparison of 3D techniques; part 6.4 p.94: Challenging materials. The main approach for small specimens $(<2 \mathrm{~cm})$ can found below.

The 3D models documented here required a digital twin mainly to ensure the maximun amount of information on the specimen's characteristics was conserved prior to destructive sampling, before shipment to other exhibitions, or to provide educational models through institutional web portals or within a museum exhibition. A small selection of these models can be found in Figs. 3.6-3.10.

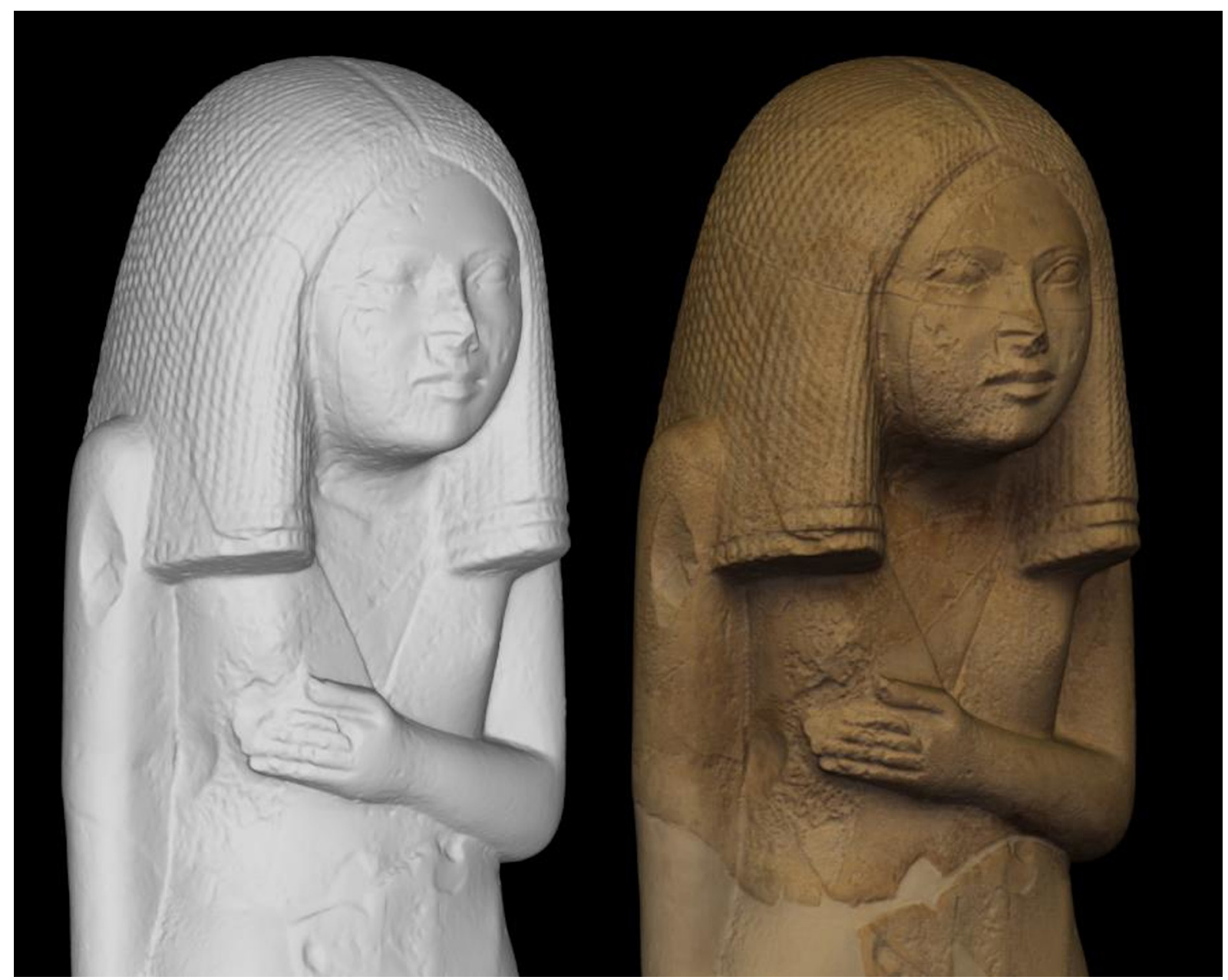

Fig. 3.6. Example of a photogrammetry model of the 'Dame de Bruxelles' (RMAH collections): on the left without the texture (colour); on the right with the texture. The model was generated in Agisoft Photoscan https://sketchfab.com/models/f19dbed309184ca299ffdb9fc8aa2c7e 


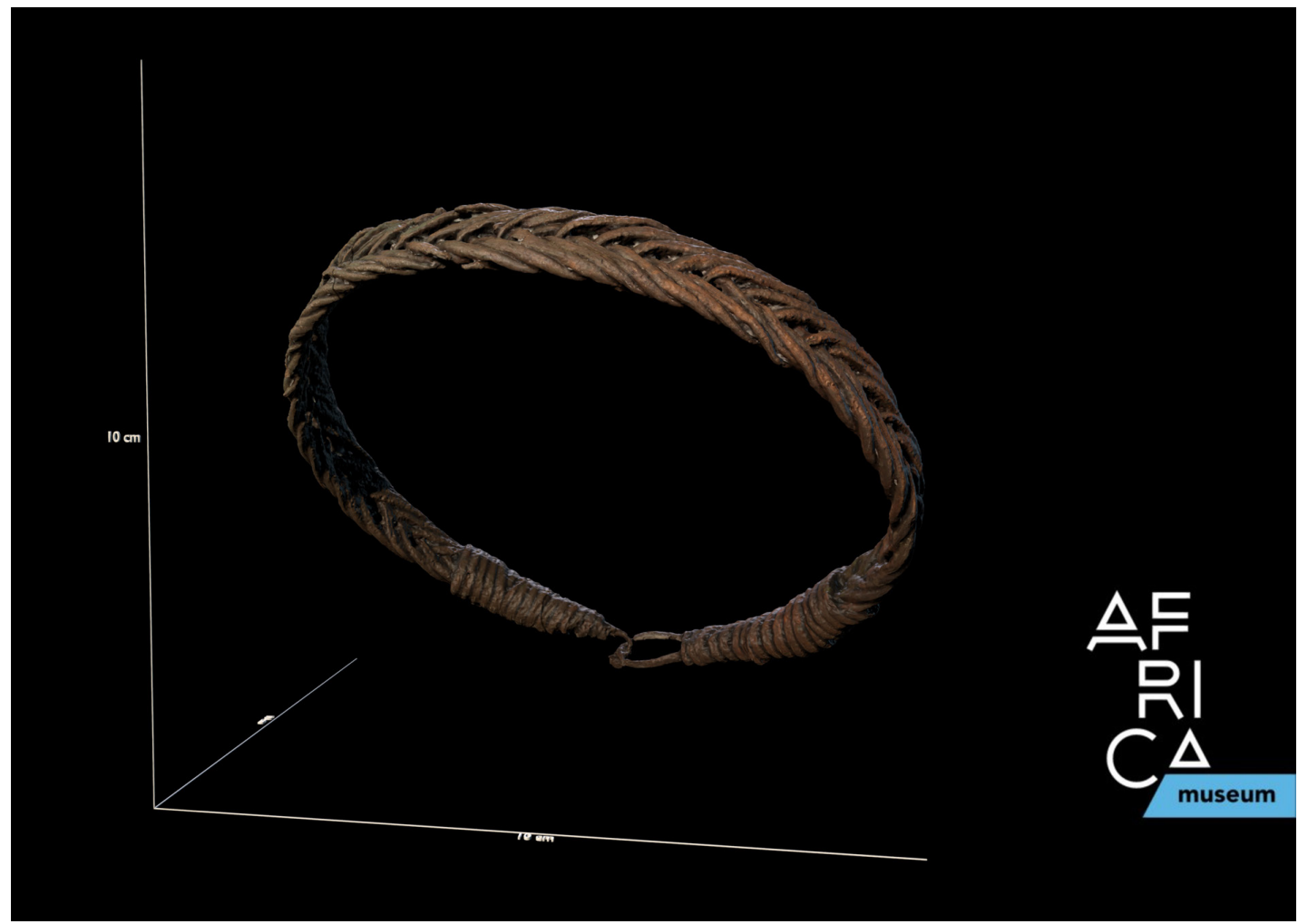

Fig. 3.7. Example of a photogrammetry model of an archaeological copper necklace from DRCongo https://sketchfab.com/models/122d9a4660a24f5181bc586672c9ffe3

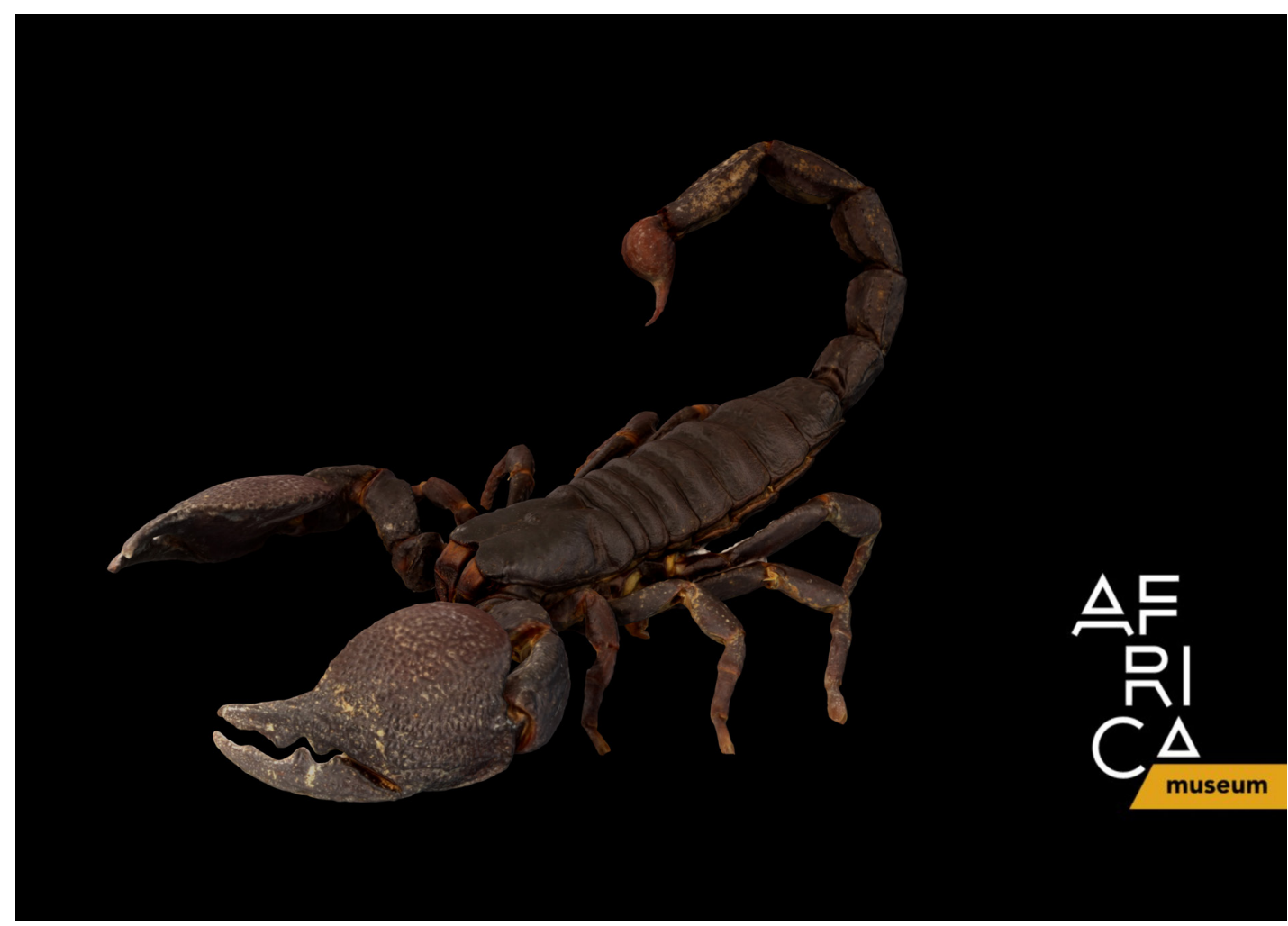

Fig. 3.8. Example of photogrammetry model of Pandinus imperator https://sketchfab.com/models/94aab6ef89a34fff866ecbd1f30747a5 


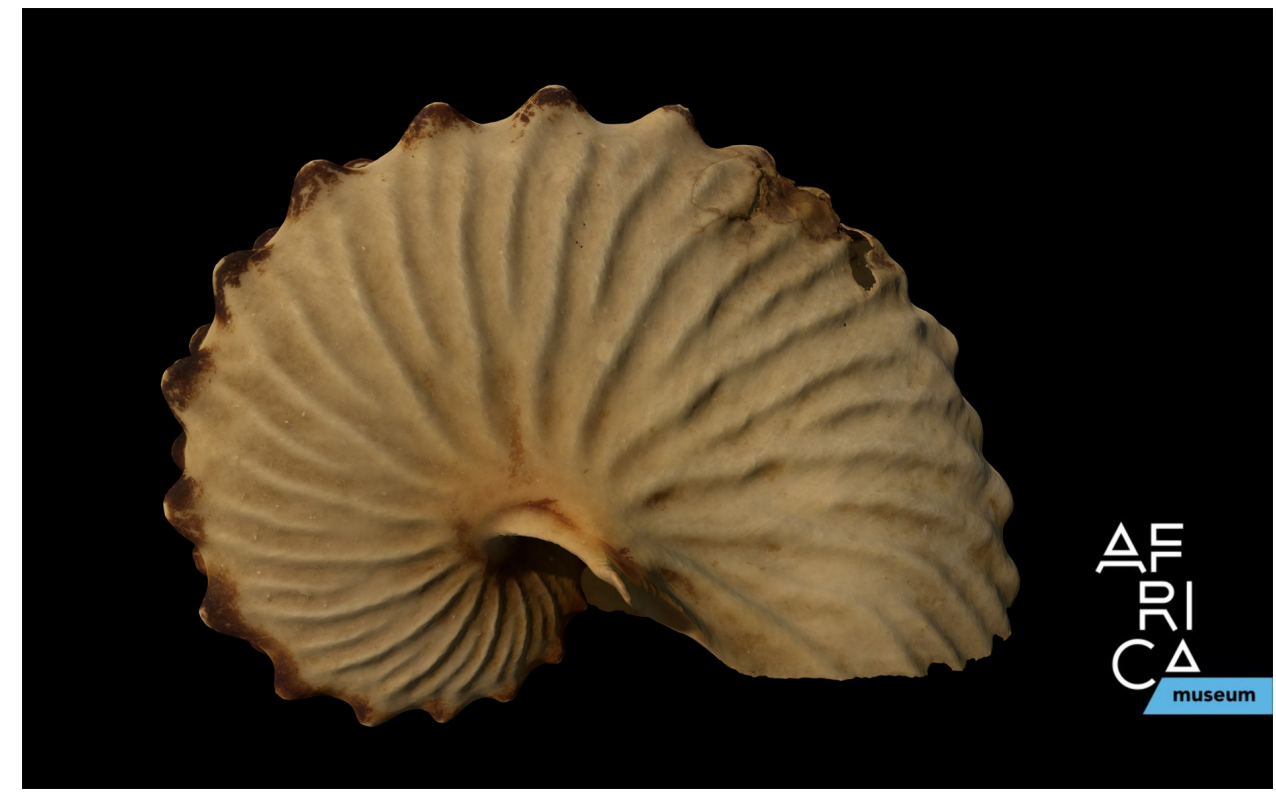

Fig. 3.9. Example of photogrammetry model of Argonauta tuberculata https://sketchfab.com/models/daed659ee685452b91d8f8c91dff761b

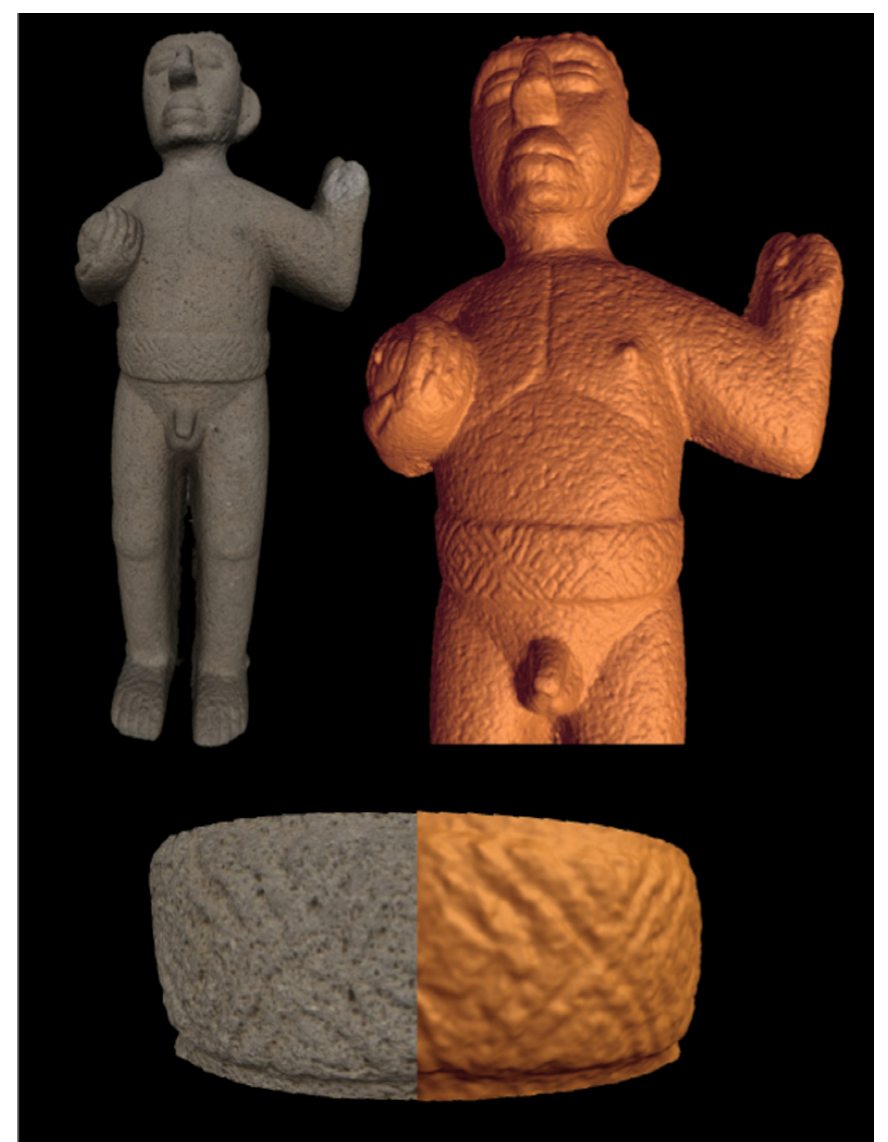

Fig. 3.10. Photogrammetry model of a Costa Rican Sacrificing Warrior (800-1300 AD) in Basalt (RMAH collections). The possibility of viewing the model without the texture has improved the visibility of the belt markings. https://sketchfab.com/models/03a9c7c61cdf48c8845498d1a6b19a73 
More examples can be found on the SketchFab accounts of both RMCA as RBINS (https://sketchfab.com/africamuseum and https://sketchfab.com/naturalsciences) The models are also integrated in our Virtual Collections pages (http://new-virtualcollections.naturalsciences.be/ and http://digit03.africamuseum.be/home)

\subsubsection{References}

Barbero-García I., Lerma J.L., Marqués-Mateu Á., Miranda P. 2017. Low-cost smartphone-based photogrammetry for the analysis of cranial deformation in infants. World Neurosurgery 102: 545-554. https://doi.org/10.1016/j.wneu.2017.03.015

Beltran R.S., Ruscher-Hill B., Kirkham A.L. \& Burns J.M. 2018. An evaluation of three-dimensional photogrammetric and morphometric techniques for estimating volume and mass in Weddell seals Leptonychotes weddellii. PLoS One 13 (1): e0189865. https://doi.org/10.1371/journal.pone.0189865

Evin A., Souter T., Hulme-Beaman A., Ameen C., Allen R., Viacava P., Larson G., Cucchi T., Dobney K. 2016. The use of close-range photogrammetry in zooarchaeology: creating accurate 3D models of wolf crania to study dog domestication. Journal of Archaeological Science: Reports 9: 87-93.

https://doi.org/10.1016/j.jasrep.2016.06.028

Falkingham P.L., Bates K.T., Avanzini M., Bennett M., Bordy E.M., Breithaupt B.H., Castanera D., Citton P., Díaz-Martínez I., Farlow J.O, Fiorillo A.R., Gatesy S.M., Getty P., Hatala K.G., Hornung J.J, Hyatt J.A., Klein H., Lallensack J.N., Martin A.J., Marty D., Matthews N.A., Meyer C.A., Milàn J., Minter N.J., Razzolini N.L., Romilio A., Salisbury S.W., Sciscio L., Tanaka I., Wiseman A.L.A., Xing L.D. \& Belvedere M. 2018. A Standard protocol for documenting modern and fossil ichnological data. Palaeontology 61 (4): 469-80. https:/doi.org/10.1111/pala.12373

Giacomini G., Scaravelli D., Herrel A., Brown R. \& Meloro C. 2017a. Bat skull in three dimensions: Photogrammetry as a portable and reliable scanning solution. $14^{\text {th }}$ European Bat Research Symposium.

Giacomini G., Scaravelli D., Herrel A., Brown R. \& Meloro C. 2017b. Echo from the skulls: Is there a trade-off between echolocation and feeding function in bats? $14^{\text {th }}$ European Bat Research Symposium.

Guery J., Hess M. \& Mathys A. 2017. Photogrammetry. In: Bentkowska-Kafel A., MacDonald L. (eds) Digital Techniques for Documenting and Preserving Cultural Heritage: 229-235. Kalamazoo and Bradford: Arc Humanities Press, Amsterdam.

Hernandez A. \& Lemaire E. 2016. A smartphone photogrammetry method for digitising prosthetic socket interiors. Prosthetics and Orthotics International 41 (2): 1-5.https://doi.org/10.1177/0309364616664150

Historic England. 2017. Photogrammetric Applications for Cultural Heritage. Guidance for Good Practice. Historic England, Swindon.

Kreij A., Scriffignano J., Rosendahl D., Nagel T. \& Ulm S. 2018. Aboriginal stone-walled intertidal fishtrap morphology, function and chronology investigated with high-resolution close-range Unmanned Aerial Vehicle photogrammetry. Journal of Archaeological Science 96: 148-161.

https://doi.org/10.1016/j.jas.2018.05.012

Lallensack J.N., van Heteren A.H. \& Wings O. 2016. Geometric morphometric analysis of intratrackway variability: a case study on theropod and ornithopod dinosaur trackways from Münchehagen (Lower Cretaceous, Germany). PeerJ 4: e2059. https://doi.org/10.7717/peerj.2059

Lautenschlager S. 2016. Reconstructing the past: methods and techniques for the digital restoration of fossils. Royal Society Open Science 3 (10): 160342. https://doi.org/10.1098/rsos.160342

Luhmann T., Robson S., Kyle S. \& Böhm J. 2013. Close-Range Photogrammetry and 3D Imaging. De Gruyter, Berlin. https://doi.org/10.1515/9783110302783 
Mallison H. \& Wings O. 2014. Photogrammetry in paleontology. A practical guide. Journal of Paleontological Techniques 12: 1-31.

Marcy A.E., Fruciano C., Phillips M.J., Mardon K. \& Weisbecker V. 2018. Low resolution scans can provide a sufficiently accurate, cost- and time-effective alternative to high resolution scans for 3D shape analyses. PeerJ 6: e5032. https://doi.org/10.7717/peerj.5032

Micheletti N., Chandler J.H. \& Lane S.N. 2014. Investigating the geomorphological potential of freely available and accessible structure-from-motion photogrammetry using a smartphone. Earth Surface Processes and Landforms 40 (4):473-486. https://doi.org/10.1002/esp.3648

Micheletti N., Chandler J.H. \& Lane S.N. 2015. Structure from Motion (SfM) Photogrammetry. In: Clarke, L.E. \& Nield, J.M. (eds) Geomorphological Techniques (Online Edition). British Society for Geomorphology, London.

Muñoz-Muñoz F., Quinto-Sánchez M. \& González-José R. 2016. Photogrammetry: a useful tool for three-dimensional morphometric analysis of small mammals. Journal of zoological systematics and evolutionary research 54 (4): 318-325. https://doi.org/10.1111/jzs. 12137

Remondino F., Rizzi A., Girardi S., Petti F.M. \& Avanzini M. 2010. 3D Ichnology-recovering digital 3D models of dinosaur footprints. The Photogrammetric Record 25: 266-282.

https://doi.org/10.1111/j.1477-9730.2010.00587.x

Remondino F. 2011. Heritage Recording and 3D Modeling with Photogrammetry and 3D Scanning. Remote Sensing 3: 1104-1138. https://doi.org/10.3390/rs3061104 


\subsubsection{SfM of small specimens $(<2 \mathrm{~cm})$}

Structure from Motion is a great tool for creating high-quality 3D models of museum collection specimens at a low cost. With decent camera equipment including a range of lenses, one can picture small to colossal specimens and convert almost an entire collection into 3D images. Like every other digitisation technique, SfM has its limits: for decent SfM models, it is absolutely essential that the object pictured is in focus with a low noise level and good lighting. Creating the light setup and setting the camera to obtain good light conditions and low noise level are relatively easy tasks. However, the infocus part depends on the focal depth created by the combination of the lens and the f-stop of the camera. This zone is very narrow when picturing tiny specimens, smaller than $2 \mathrm{~cm}$ (but modelling specimens up to $\sim 4 \mathrm{~cm}$ can also benefit from focus stacking), at the lens' optimal f-stop. Therefore, SfM 3D models of specimens smaller than $2 \mathrm{~cm}$ tend to have a lot of noise due to the large unfocused areas (Fig. 3.11). Stopping down the lens (increasing the f-number) will increase depth of field, but past the point of the optimal aperture, diffraction will cause the image to lose sharpness.

The only option to picture small objects at the lens optimum with a large depth of field is to apply focus stacking for each view of the object. The only downside is that this will increase the time of the acquisition by the number of pictures needed to increase the depth of field. Often 15-30 pictures per view are necessary to obtain sufficient depth of field. When a normal SfM acquisition would take around $20 \mathrm{~min}$ this would mean it now takes $300-600 \mathrm{~min}$. This is too long to even consider this technique. But a few things can be changed to digitise a specimen at faster speed. Recently, a fully automatic system (DISC3D by Ströbel et al. 2018) was developed that records 2D+ images from 398 viewing angles (leading to approx. $10^{\circ}$ between camera positions) in less than 3 hours. The all-side imaging scheme (Fig 3.12) allows 3D modelling of very small specimens such as Sitophilus oryzae (3.8 mm body size, Fig 3.12), but also objects with complex surface structures such as a black elder (Alnus japonica) cone (Fig 3.13). Adding more viewing angles (e.g., $7^{\circ}$ between camera position results in 807 EDOF.images)

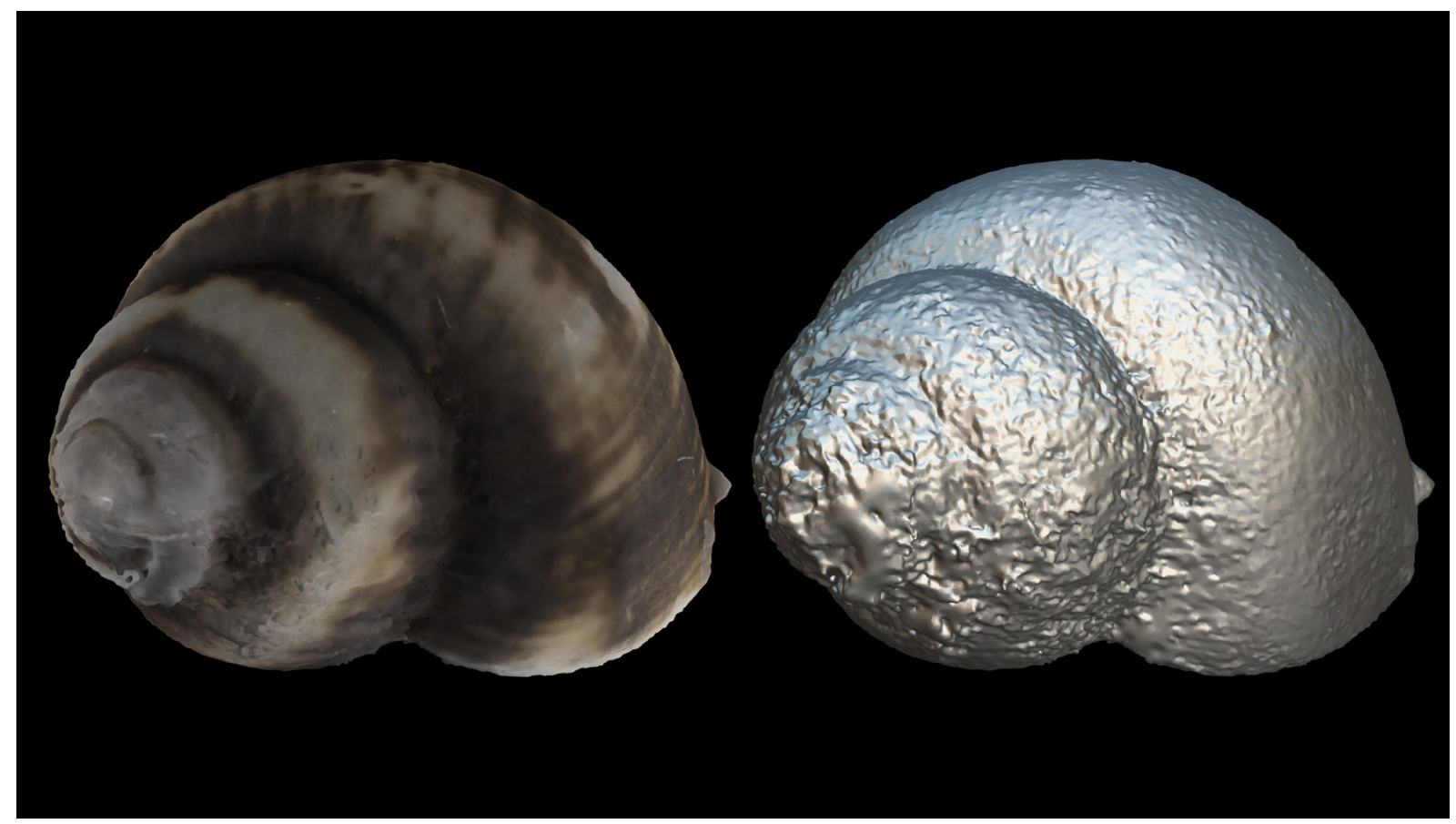

Fig. 3.11. 3D model, with texture on the left and mesh only on the right, of the mollusk Gabbiella humerosa edwardi (size: $6 \mathrm{~mm}$ ) generated in Agisoft Photoscan based on single images (f/14). https://sketchfab.com/models/73c74dc0d17b4d5f99b4fe4393f05302 
A

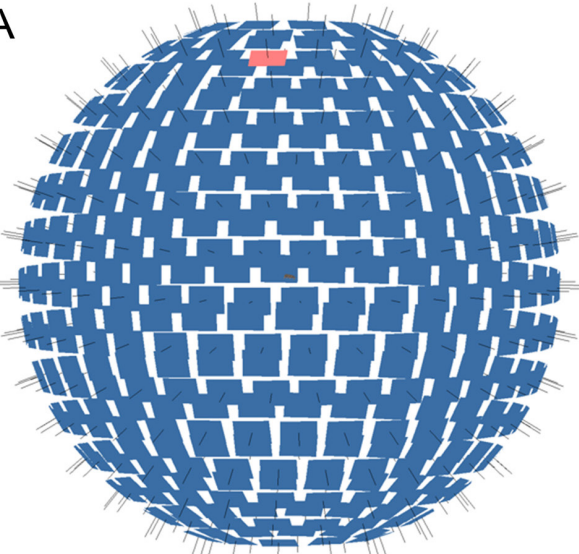

C

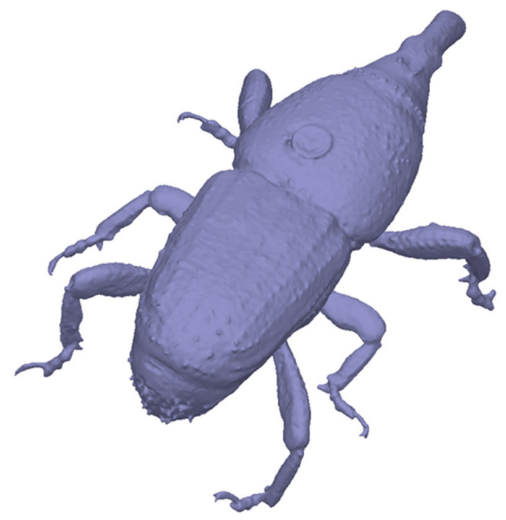

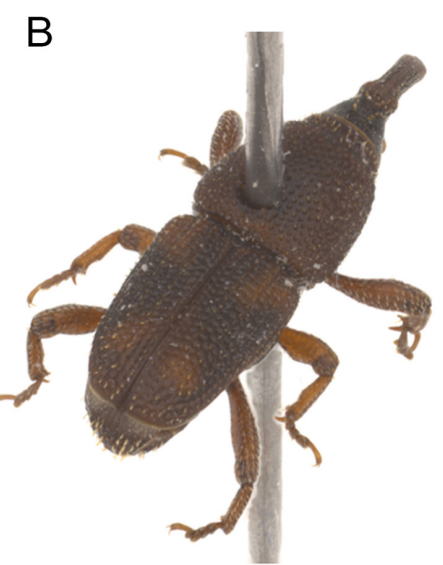

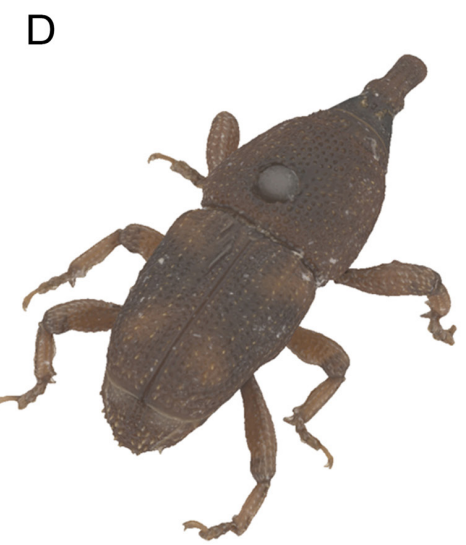

Fig. 3.12. Sitophilus oryzae ( $3.8 \mathrm{~mm}$ body size) scanned with DISC3D. A. Scanning scheme with 398 camera positions. B. EDOF-image from the red camera position. C. Vcm-mesh ( $\sim 250 \mathrm{k}$ polygones). D. Textured model.
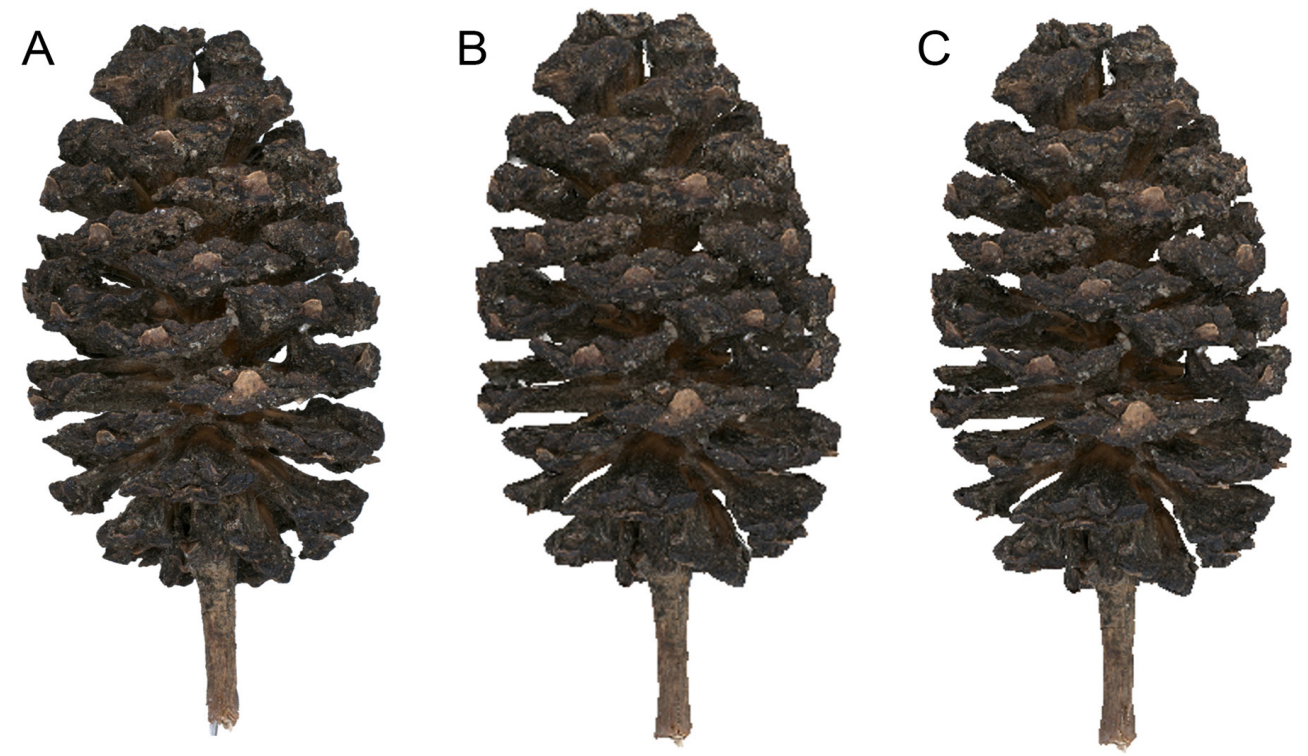

Fig. 3.13. Alnus japonica ( $2 \mathrm{~cm}$ long) scanned with DISC3D. A. EDOF-image. B. 3D-model (vcm) from 807 cameras. C. 3D-model from 398 cameras. 
more than doubles the image acquisition time and increases data processing time in Agisoft Photoscan from 2 hrs 30 mins -8 hrs 40 mins. Since this does not lead to more precision in the model geometry (Fig. 3.13), we suggest that $10^{\circ}$ differences between camera angles are suitable for most objects.

In normal SfM studio lights are often used instead of flashes. In focus stacking setups, flashes are often used to reduce the acquisition time per image and to reduce sample movement, because in most consumer-grade cameras it is not possible to perform a mirror lockup prior to taking a picture. Therefore, when combining focus stacking and SfM, it is better to choose flashes over a continuous light source. This will further decrease the acquisition time, but $1-3 \mathrm{hrs}$ is still usually required to picture an object from all the necessary angles required for a good SfM model (Fig. 3.14).

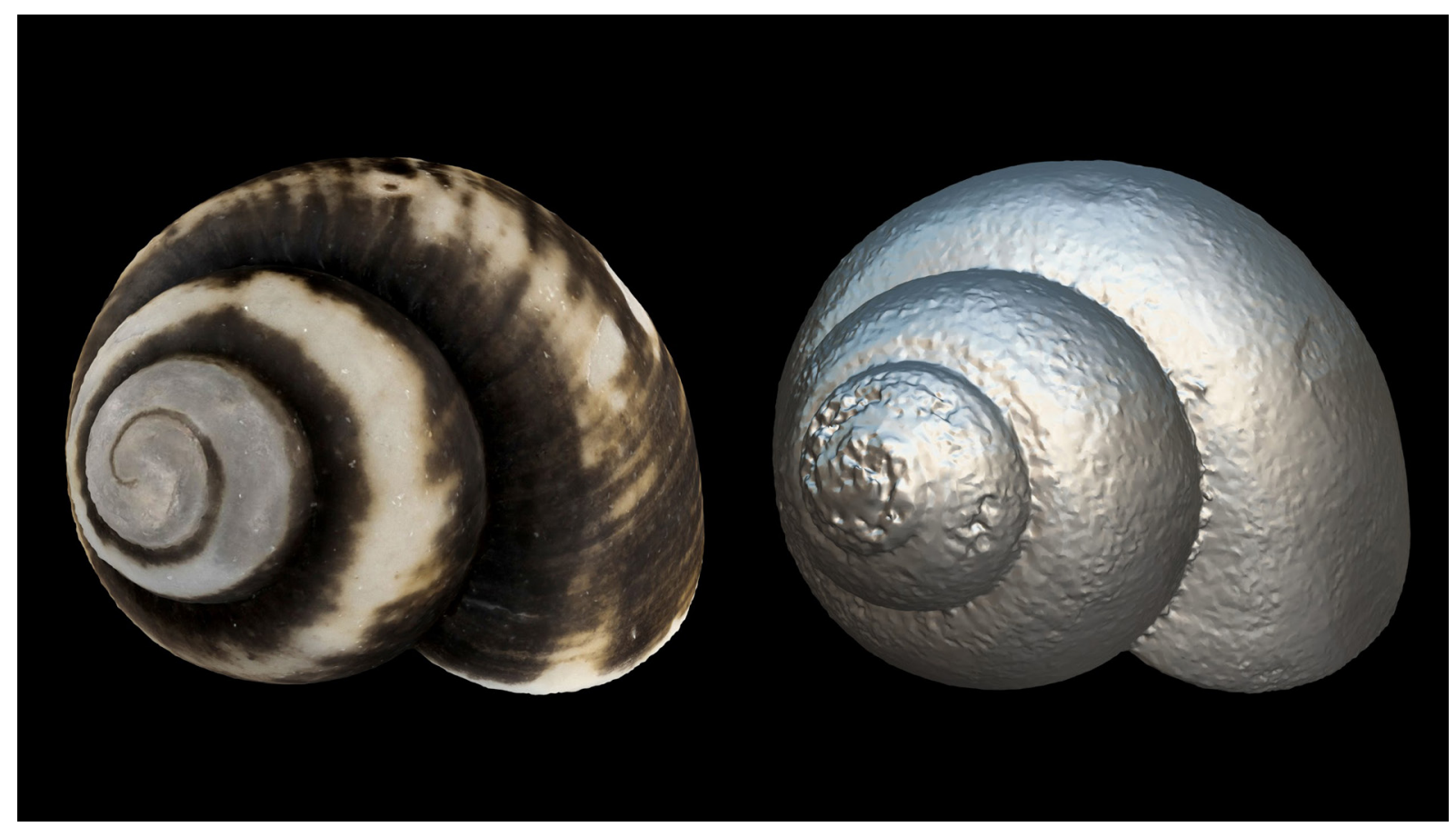

Fig. 3.14. 3D model, with texture on the left and Matcap on the right, of the mollusk Gabbiella humerosa edwardi (size: $6 \mathrm{~mm}$ ) generated in Agisoft Photoscan based on focus-stacked images. https://sketchfab.com/models/0a8aea3251244978af7a3f4f95bca881

Although focus stacking SfM is time-consuming, it is still worth the invested time, especially if the setup can run in an automated way, such as with DISC3D. The idea of combining the two techniques is not entirely new, but most of the software used in previous years did not give satisfactory results or completely failed to calculate a model out of stacked images. This is often due to the fact that most stacking software fails produce EDOF-images with a correct perspective regarding the pin-hole camera model, which is solved in the DISC3D stacking algorithm (Ströbel et al. 2018). Within a natural history context, the technique has not been used or tested a lot (Nguyen et al. 2014 (SFS); Brecko et al. 2014; Santella \& Milner 2017; but see Ströbel et al. 2018) for several reasons. Firstly, the single pictures often show more detail than the 3D model, which is very important in taxonomic studies, while the 3D model is key to morphometric measurements and as an educational tool for the general public. Also, due to the lack of fine detail, the resulting models cand lack the quality needed for geometric morphometric or biomechanical studies. Lastly, $\mu \mathrm{CT}$ is routinely used to compose 3D models of small specimens and artefacts. However, in archaeological and cultural heritage studies, the technique has proven helpful for documenting fine details like cut marks (Maté Gonzales et al. 2015, 2017; Mathys et al. in prep), artefacts 
and engravings (Plisson 2015; Plisson \& Zotkina 2015; Dubreuil et al. 2015; Marziali \& Dionisio 2017; Kontogianni et al. 2017), etc., as these objects are fairly easy to capture given they are composed of a relatively smooth surface with deeper features.

\subsubsection{Material and methods}

The focus-stacking component is best executed using an automated camera rail (e.g., Cognisys StackShot (https://www.cognisys-inc.com/products/stackshot/stackshot.php), WeMacro(http://www.wemacro.com/), MJKZZ (https:/www.mjkzz.com/product-page/qool-rail-250) or similar) to make sure the pictures are taken at fixed step-size intervals (Table 3.2). For the SfM part it is necessary that the object turns around

Table 3.2. Equipment list needed to perform photogrammetry of small specimens.

\begin{tabular}{c}
\hline DSLR \\
\hline Flashes with extra external battery or AC/DC connection \\
Macro Lens 1:1 \\
Macro Lens 1-5x (Canon) or 2.5-5x (Laowa) \\
Stackshot 3X Deluxe Kit (Stacking Rail + 2 turntables) \\
Zerene Stacker \\
Agisoft Photoscan
\end{tabular}

to complete a $360^{\circ}$ rotation from several angles so in essence pictures are taken from a sphere around the specimen. This can be done manually, using a microscope rotary table, or automatically with a stepper motor controlled rotary table. At RBINS and RMCA the digitisation process has been automated even more by adding a second rotary table (https://www.cognisys-inc.com/products/stackshot3x/stackshot3x.php). This one controls the position of the rotary table which holds the specimen. They are fixed perpendicularly together with a metal corner. Fixing them together like this makes it possible to move the angle of the specimen and rotate it $360^{\circ}$ (Fig. 3.15). Combined with a stacking rail, it offers the possibility to obtain
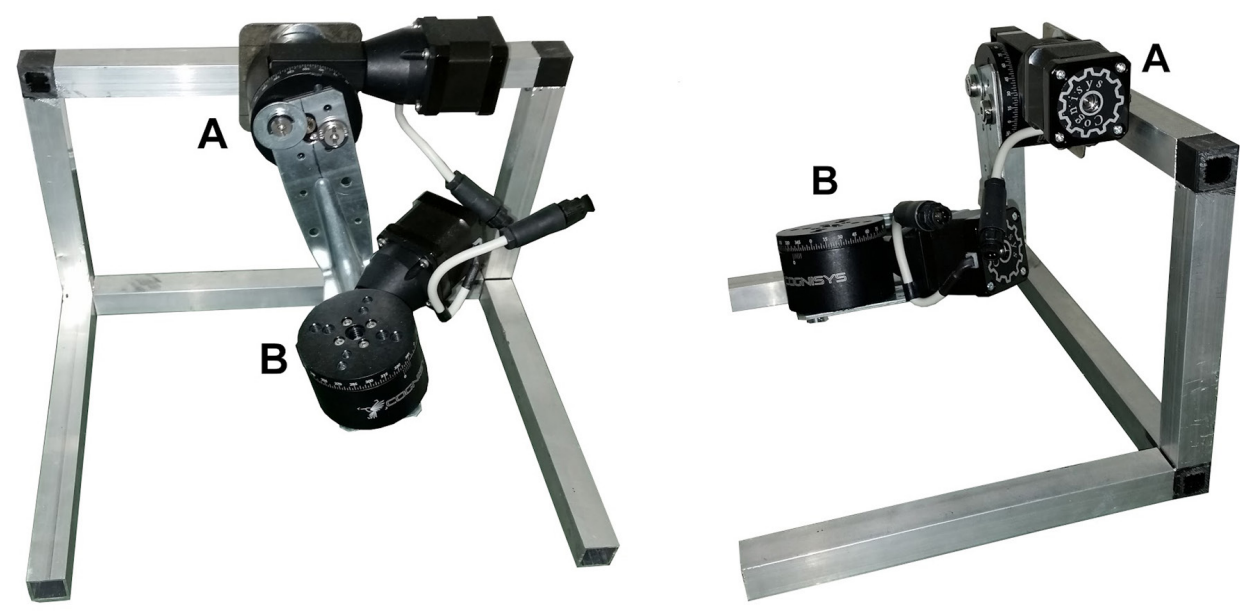

Fig. 3.15. Part of the Cognisys StackShot $3 X$ Deluxe Kit, reassembled for the photogrammetry purpose. The two rotary tables are mounted perpendicular to each other, whereby rotary table A moves a steel angle with rotary table B fixed at the end. 
stacked images for each view, including at different angles, reducing the human interaction time needed to centre the specimen, set the step size and the number of rotations.

In the RBINS/RMCA digitisation cell a consumer-grade DSLR is used equipped with macro objectives (Canon EF-S 60 mm F2.8 USM Macro or Canon MP-E 65 mm F2.8 1-5× Macro Photo). However, recent studies have proved that using a machine vision camera can speed up the capturing process considerably (Ströbel et al. 2018).

The software used to stack the pictures is Zerene Stacker. However, unlike other available stacking software, the algorithm used by Ströbel et al. (2018) takes the perspective into account, which is important to obtain aberration-free images. To compute the SfM 3D model, Agisoft Photoscan (Agisoft Metashape) is used. If calculating the 3D surface of objects with fine detail (like hairs, wings, insect legs, claws, etc.), it is best to use the visibility-consistent meshing (VCM) option as this results in meshes capturing such fine details (Ströbel et al. 2018). An automatic imaging system, DISC3D (Ströbel et al. 2018), is also available as an open source system or pre-assembled. It can be equipped with different cameras (the system uses Basler cameras with 4- and 12-megapixel resolution sensors). The higher resolution results in $2 \mathrm{D}+$-images with more detail, but their use for SfM is problematic (see Table 3.3 and Fig 3.16).

Table 3.3. DISC3D camera comparison for processing 398 2D + images for model generation in Agisoft Photoscan Pro 1.4.5 (build 7354).

\begin{tabular}{ccc}
\hline & 4MP sensor & 12 MP sensor \\
\hline Camera alignment & 3 minutes & 45 minutes \\
Depth maps (Ultra high) & 47 minutes & 10 hours \\
Dense cloud (Ultra high) & 5 hours & 74 hours \\
N points in dense cloud & 4.2 million & 32.3 million \\
VCM meshing & 68 minutes & N/A (software crashes) \\
\hline
\end{tabular}

\subsubsection{Collections}

The extended depth of field is only necessary when the depth of field (using the optimal parameters of the camera and lens) on a single image is insufficient (Fig 3.17). This is generally only the case when dealing with objects/specimens smaller than $2 \mathrm{~cm}$ in diameter. When possible (time and availability of an automatic system), imaging of larger specimens in the range of up to $4 \mathrm{~cm}$ also benefits from focus stacking. Such tiny objects are found in every collection, but most are likely situated in Entomology, Recent Invertebrate and Palaeontology collections.

To test whether the technique copes well with small translucent objects, a small shell of the mollusk Anceya giraudi was digitised. The general shape and texture of the resulting image is good, without any detectable noise caused by the lighting technique (Fig. 3.18)

Digitising insects is more complicated because of the small legs and fine details. The general shape of an ant is beautifully captured, although the fine detail is lacking. Also, due to a little overexposure on the head of the ant, a bit of noise is created. It might be possible to avoid this using polarised filters (Fig 3.19). 

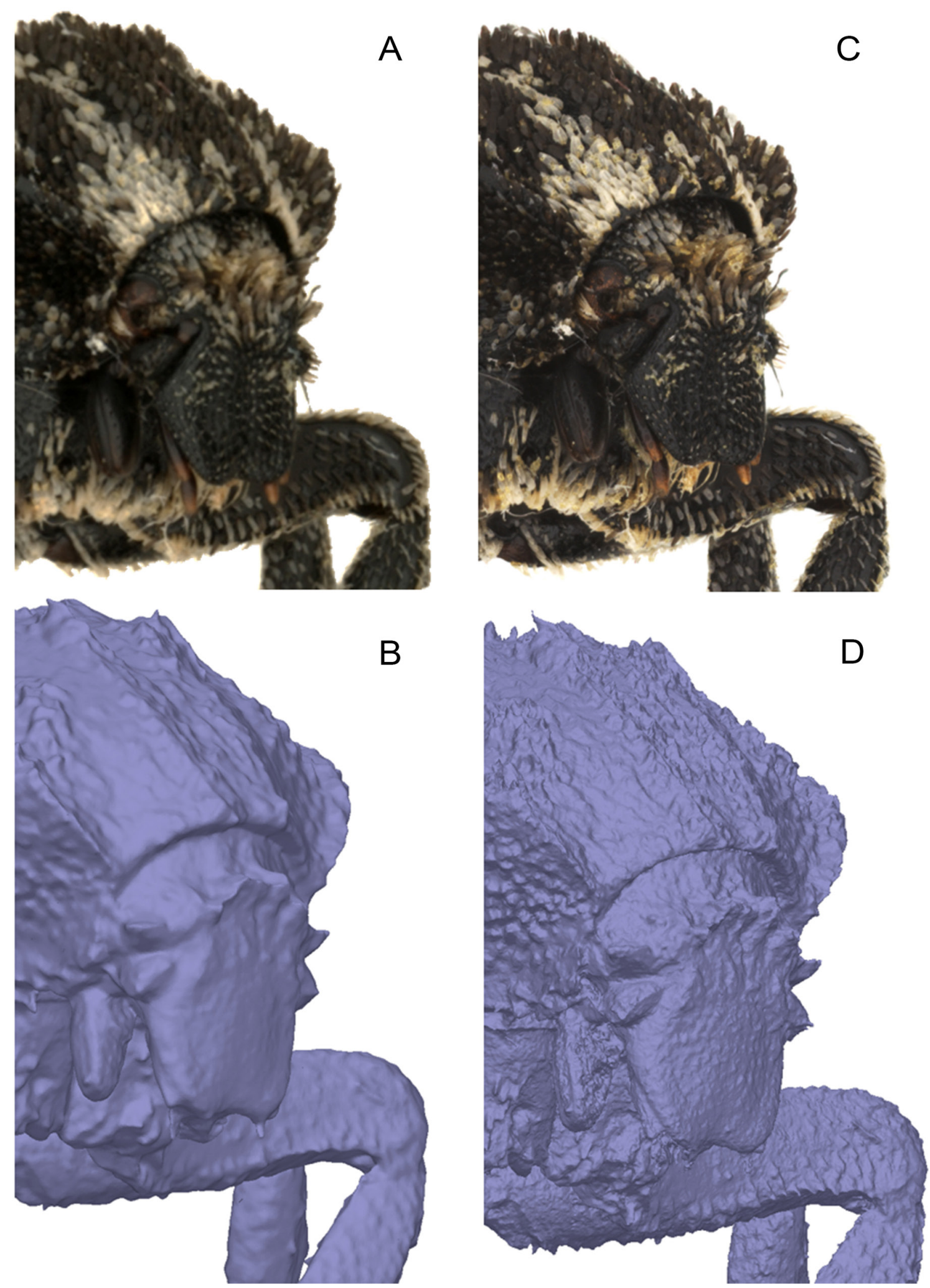

Fig. 3.16. Valgus hemipterus ( $7 \mathrm{~mm}$ body size) scanned with DISC3D at two different camera resolutions. A-B. 4 MP. C-D. 12 MP. Both images are sharp, but the $12 \mathrm{MP}$ version shows more detail, resulting also in a higher spatial resolution of the model (but see Table 3.3). The textured VCM-model (low polygonversion) can be inspected at Sketchfab: https://skfb.ly/6KpsB 


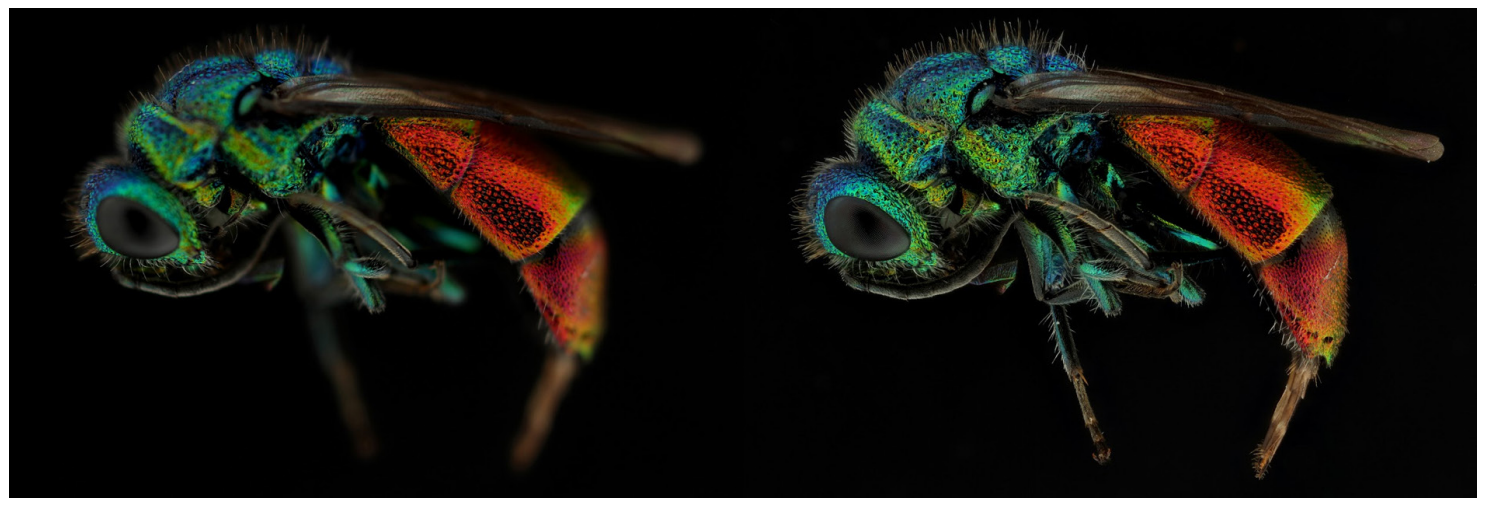

Fig. 3.17. There is insufficient depth of field in the left-hand image. Focus stacking a range of images of the same viewpoint and parameters at different distances results in the right-hand image, where sufficient depth of field to perform photogrammetry is present.

When reprocessing the ant with the VCM method, the model is a lot cleaner. Most of the noise is gone and the details are much sharper. Also, the spurs on the tibia are now visible, whereas they were absent in the previous calculations. This demonstrates the interest of the method for calculating models of insects or specimens with fine details (Fig 3.20).

Another insect that was pictured to test this technique is a specimen of the beetle Omorgus gigas. It was chosen because it is at the limit of what is possible to digitise with regular SfM before requiring high detail structured light scanning, which is discussed later on in this volume. The scan obtained by focus stacking SfM is also compared to other techniques and $\mu \mathrm{CT}$ scanning in Chapter 6: Comparison of 3D

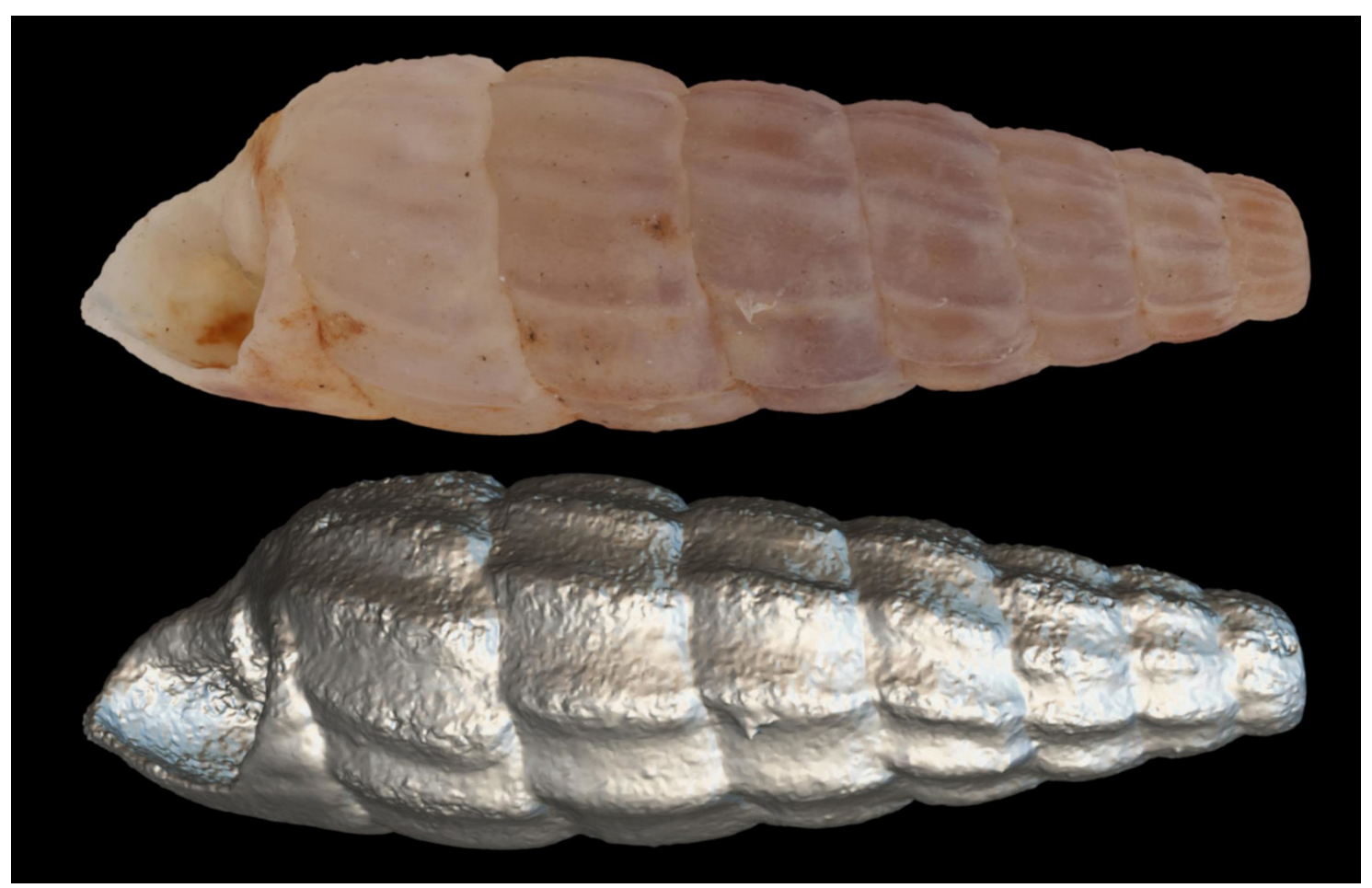

Figu. 3.18. 3D model (top image with texture; bottom image with mesh only), of the mollusk Anceya giraudi generated in Agisoft Photoscan based on focus-stacked images. The size of the shell is $9 \mathrm{~mm}$. https://sketchfab.com/models/47af5ea5aad14168924ecb72c44af05a 


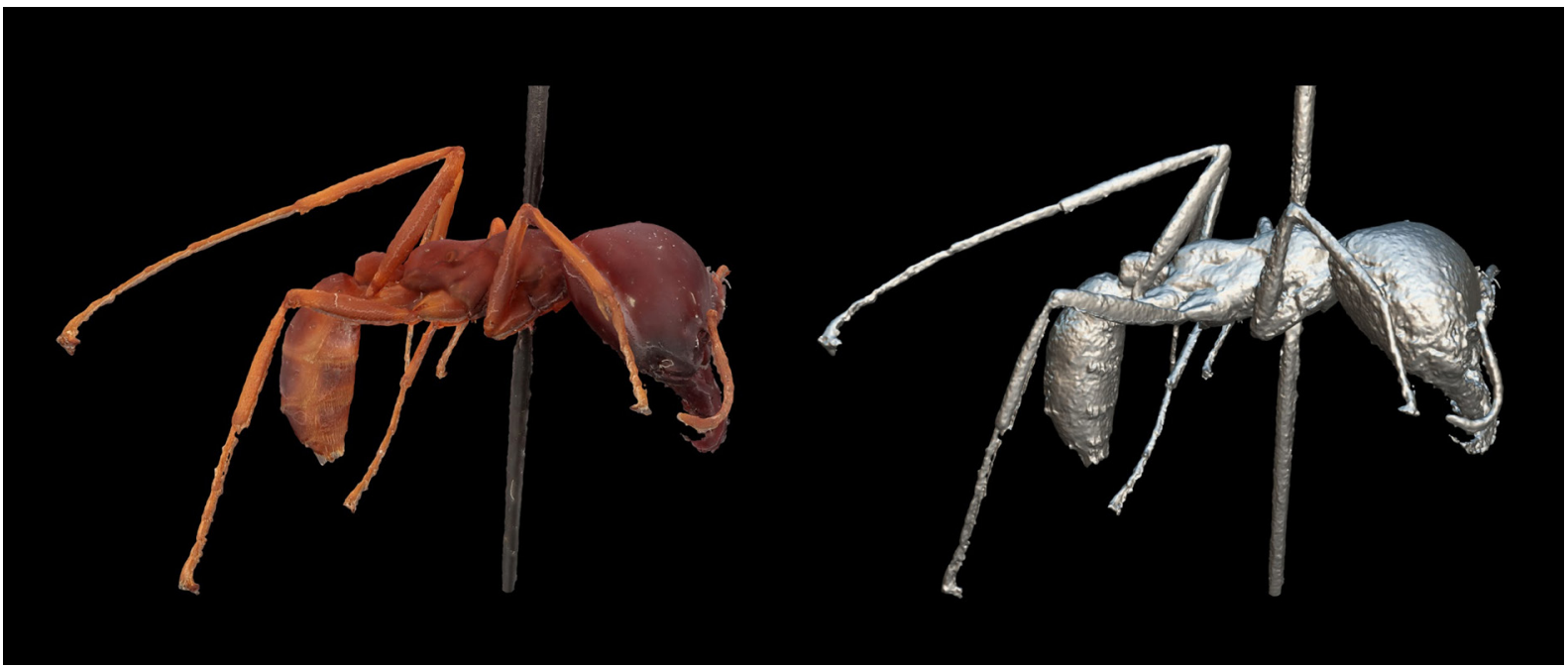

Fig. 3.19. 3D model of a Dorylus ant (size: $1.5 \mathrm{~cm}$ ) based upon focus stacked images, textured model is on the left, the view of only the mesh is on the right.

https://sketchfab.com/models/c6c1bd08cb724f85a8bd8aecc6c44d7e

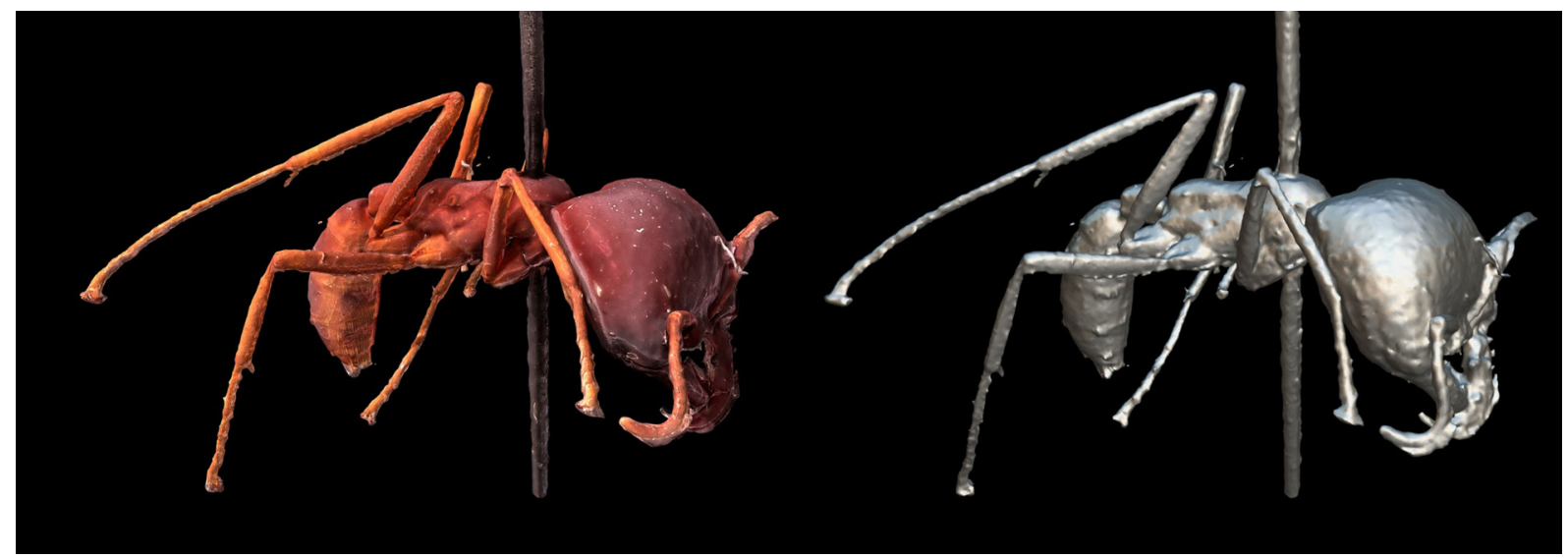

Fig. 3.20. 3D model of a Dorylus ant (size: $1.5 \mathrm{~cm}$ ) based upon focus stacked images, textured model is on the left, the view of only the mesh is on the right. The VCM option in Agisoft Photoscan is chosen to include small detail in the 3D model. The tibia spurs are clearly marked. https://sketchfab.com/models/da9aa414bfa64caabfe5c552368b16f0

techniques (p. 85). The focus stacking SfM model calculated with the VCM method shows a lot of detail and there is no noise as is expected for an object that is quite matt. The tricky parts for this specimen are the black bulges on the carapace and the small details between them. These are all clearly visible. Thanks to the focus stacked images the details like the claws are preserved on the 3D model (Fig. 3.21).

\subsubsection{Price of the system}

The complete price for the Stackshot setup, the Cognisys 3X system, the Agisoft Photoscan license and a license for Zerene Stacker, is approximately 5500 euros (price DISC3D, see Ströbel et al. 2018 or direct contact with the authors). Included are the prices of an Agisoft Photoscan Professional educational license and the professional edition of the Zerene Stacker too. The price for a computer is not included as this depends heavily on the configuration, but normally for around 1000-1500 euros a decent machine can be bought that is capable of computing 3D models. In Table 3.4, the price per week of the staff/ 


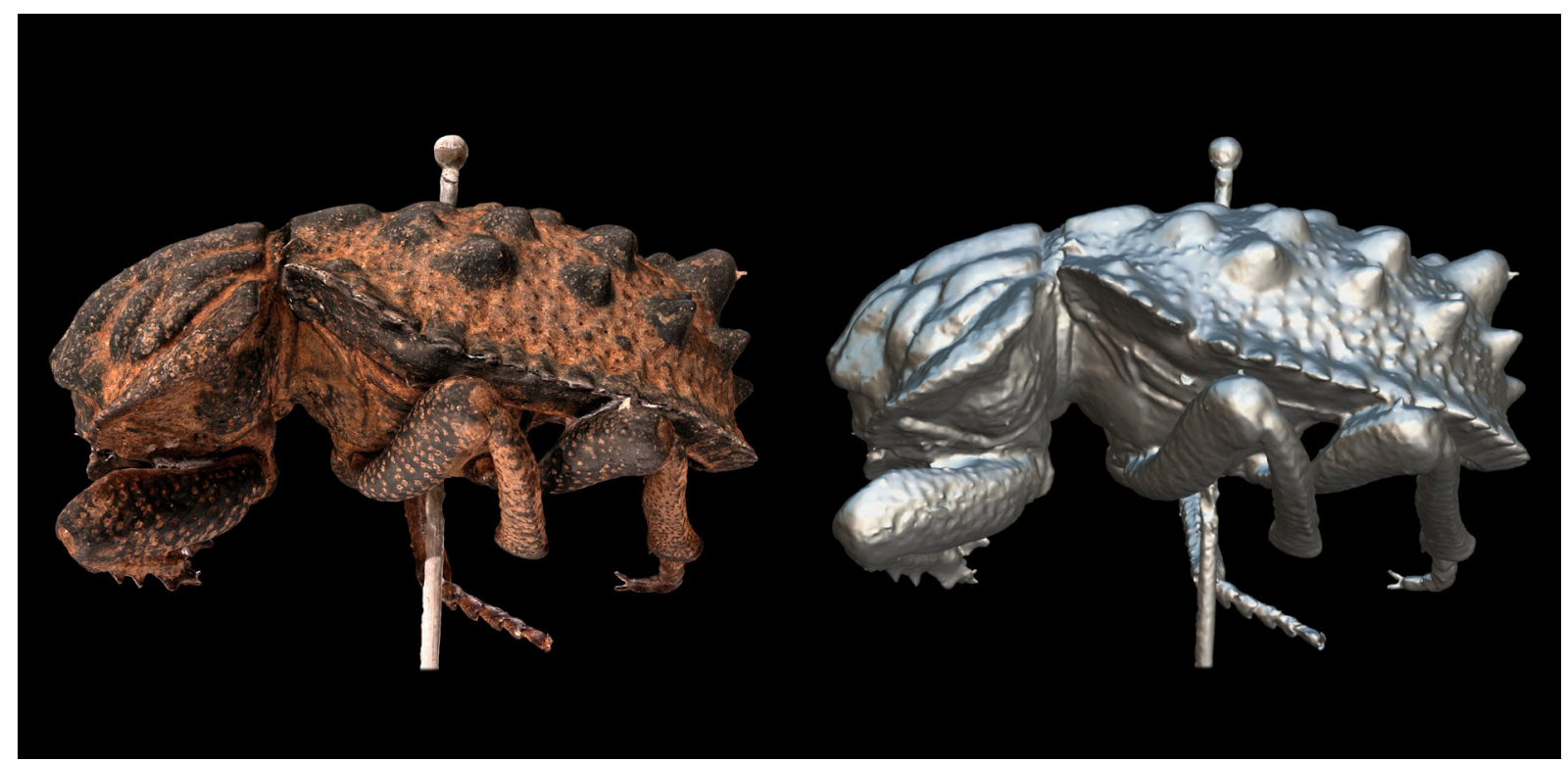

Fig. 3.21. 3D model of an Omorgus gigas by using focus stacked images and the VCM method in Agisoft Photoscan. https://sketchfab.com/models/ad5fd157c5424c8fb793c1c282450d33

operator is included as the cost to operate the machine. Of course, this is different in every country/ institution and depends on whether a scientist or technician operates the equipment. But it gives a good indication of the running cost after purchasing the equipment. With the setup it is possible to digitise 10 more-or-less complex specimens (like an ant) per week as this requires 5 rotations minimum to cover the entire specimen. To digitise simple forms like a shell 3 rotations might be enough, making it possible to scan 20 specimens per week. Because it takes approximately 20 minutes before a rotation is finished, it is possible to start a second machine to perform focus stacking of another specimen. Therefore, a part in the table that considers the price of two entire set-ups (software included) is added and the number of specimens that can be pictured in a week is doubled. Furthermore, the price evolution is shown for each model depending on the target number of models and also the time it takes to digitise the total number of models. It is quite clear that if a collection contains less than 100 specimens, there is no need to double the setup as the price per digitised specimen will not change much, only the time required to do it would be halved. A collection of 500 specimens or more is the point where it is interesting to consider adding a second setup, as this not only divides the digitisation time by half, but also reduces considerably the cost per specimen.

\subsubsection{References}

Brecko J., Mathys A., Dekoninck W., Leponce M., Van den Spiegel D., \& Semal P. 2014. Focus stacking: comparing commercial top-end set-ups with a semi-automatic low budget approach. A possible solution for mass digitisation of type specimens. ZooKeys 464: 1-23. https://doi.org/10.3897/zookeys.464.8615

Kontogianni G., Chliverou R., Koutsoudis A., Pavlidis G. \& Georgopoulos A. 2017. Enhancing close-up image based $3 \mathrm{~d}$ digitisation with focus stacking. ISPRS - International Archives of the Photogrammetry, Remote Sensing and Spatial Information Sciences XLII-2/W5: 421-425. https://doi.org/10.5194/isprs-archives-XLII-2-W5-421-2017

Marziali S. \& Dionisio G. 2017. Photogrammetry and macro photography. The experience of the MUSINT II Project in the 3D digitising process of small size archaeological artifacts. Studies in Digital Heritage 1 (2): 298-309. https://doi.org/10.14434/sdh.v1i2.23250 
Table 3.4. Overview of the digitisation costs (euro) for one or two running setups, with respectively $10 / 20$, or 20/40 digitised specimens per week, given per collection size $(20,100,500,1000$ or 10000 specimens).

\begin{tabular}{|c|c|c|c|c|c|}
\hline \multicolumn{6}{|c|}{ Digitisation with one setup } \\
\hline 10/week & 20 specimens & 100 specimens & 500 specimens & $1 \mathrm{~K}$ specimens & 10K specimens \\
\hline \# weeks & 2 & 10 & 50 & 100 & 1000 \\
\hline Running cost/week & 1000 & 1000 & 1000 & 1000 & 1000 \\
\hline Setup price & 5500 & 5500 & 5500 & 5500 & 5500 \\
\hline Total price & 7500 & 15500 & 55500 & 105500 & 1005500 \\
\hline Price/specimen & 375 & 155 & 111 & 105.5 & 100.55 \\
\hline 20/week & 20 specimens & 100 specimens & 500 specimens & $1 \mathrm{~K}$ specimens & 10K specimens \\
\hline \# weeks & 1 & 5 & 25 & 50 & 500 \\
\hline Running cost/week & 1000 & 1000 & 1000 & 1000 & 1000 \\
\hline Setup price & 5500 & 5500 & 5500 & 5500 & 5500 \\
\hline Total price & 6500 & 10500 & 30500 & 55500 & 505500 \\
\hline Price/specimen & 325 & 105 & 61 & 55.5 & 50.55 \\
\hline \multicolumn{6}{|c|}{ Digitisation with two setups } \\
\hline 20/week & 20 specimens & 100 specimens & 500 specimens & $1 \mathrm{~K}$ specimens & 10K specimens \\
\hline \# weeks & 1 & 5 & 25 & 50 & 500 \\
\hline Running cost/week & 1000 & 1000 & 1000 & 1000 & 1000 \\
\hline Setup price & 11000 & 11000 & 11000 & 11000 & 11000 \\
\hline Total price & 12000 & 16000 & 36000 & 61000 & 511000 \\
\hline Price/specimen & 600 & 160 & 72 & 61 & 51.1 \\
\hline 40/week & 20 specimens & 100 specimens & 500 specimens & $1 \mathrm{~K}$ specimens & 10K specimens \\
\hline \# weeks & 0.5 & 2.5 & 12.5 & 25 & 250 \\
\hline Running cost/week & 1000 & 1000 & 1000 & 1000 & 1000 \\
\hline Setup price & 11000 & 11000 & 11000 & 11000 & 11000 \\
\hline Total price & 11500 & 13500 & 23500 & 36000 & 261000 \\
\hline Price/specimen & 575 & 135 & 47 & 36 & 26.1 \\
\hline
\end{tabular}


Maté González M.Á., González M.Á.M., Yravedra J., González-Aguilera D., Palomeque-González J.F. \& Domínguez-Rodrigo M. 2015. Micro-photogrammetric characterization of cut marks on bones. Journal of Archaeological Science 62: 128-142. https://doi.org/10.1016/j.jas.2015.08.006

Maté González M.A., Aramendi J., González-Aguilera D. \& Yravedra J. 2017. Statistical Comparison between low-cost methods for 3D characterization of cut-marks on bones. Remote Sensing 9 (9): 873. https://doi.org/10.3390/rs9090873

Mathys A., Semal P., Brecko J., Bello S.M., Van den Spiegel D. \& Abrams G. In preparation. An overview of the advantages and limitations of 9 different methods to analyse bone retouchers.

Nguyen C.V., Lovell D.R., Adcock M. \& La Salle J. 2014. Capturing natural-colour 3D models of insects for species discovery and diagnostics. PLOS ONE 9 (4): 1-11.

https://doi.org/10.1371/journal.pone.0094346

Plisson H. 2015. Digital photography and traceology, from 2D to 3D. In: Lozovskaya O.V., Lozovski V.M. \& Girya E. (eds) Traces in the history. Dedicated to 75 anniversary of Viacheslav E. Shchelinsky. IIMKRAN, Saint Petersbourg.

Plisson H. et Zotkina L. V. 2015. From 2D to 3D at macro- and microscopic scale in rock art studies. Digital Applications in Archaeology and Cultural Heritage 2 (2-3): 102-119.

https://doi.org/10.1016/j.daach.2015.06.002

Dubreuil L., Savage D., Delgado-Rick S., Plisson H., Stephenson B., De La Torre I. 2015. Current Analytical frameworks for studies of use-wear on ground stone tools. In: Marreiros J.M., Gibaja Bao J.F. \& Bicho N. (eds) Use-Wear and Residue Analysis in Archaeology.Manuals in Archaeological Method, Theory and Technique: 105-158. Springer Nature, Cham.

Santella M. \& Milner A.R.C. 2017. Coupling focus stacking with photogrammetry to illustrate small fossil teeth. Journal of Paleontological Techniques 18: 1-17.

Ströbel B., Schmelzle S., Bluethgen N. \& Heethoff M. 2018. An automated device for the digitisation and 3D modelling of insects combining extended-depth-of-field and all-side multi-view imaging. ZooKeys 759: 1-27. https://doi.org/10.3897/zookeys.759.24584 


\subsection{Structured Light Scanning}

Structured light scanning consists of projecting light patterns on an object with a projector, which are recorded by one or multiple cameras. The captured deformation of the patterns on the object enables the software to recreate the surface of the object by triangulation (Chen et al. 2000; Pavlidis et al. 2007; Sansoni et al. 2009). Several companies manufacture a wide range of these scanners. Even within the product range of a single manufacturer there are low- and high-end scanners, which are often intended to scan large or small objects. In this chapter results of the structured light scanners present at RBINS are discussed, however this will most likely be more-or-less the same for structured light scanners of other manufacturers. While some scanners are handheld (Johnston et al. 2013; Zachar et al. 2017), the ones used at RBINS are mounted on a tripod.

Fringe projection is a specific form of structured light and the most commonly used in commercial 3D scanners. It is based on a sine wave variation of multiple fringes onto the object (Fig. 3.22) (Bell et al. 2016). Fringe projection systems generate $3 \mathrm{D}$ coordinates for each pixel resulting in a high-resolution scan (Rieke-Zapp \& Royo 2017). Structured light scanners generate either a dense point cloud or a triangulated mesh and they can use different types of light (white, red, green, blue, infrared).

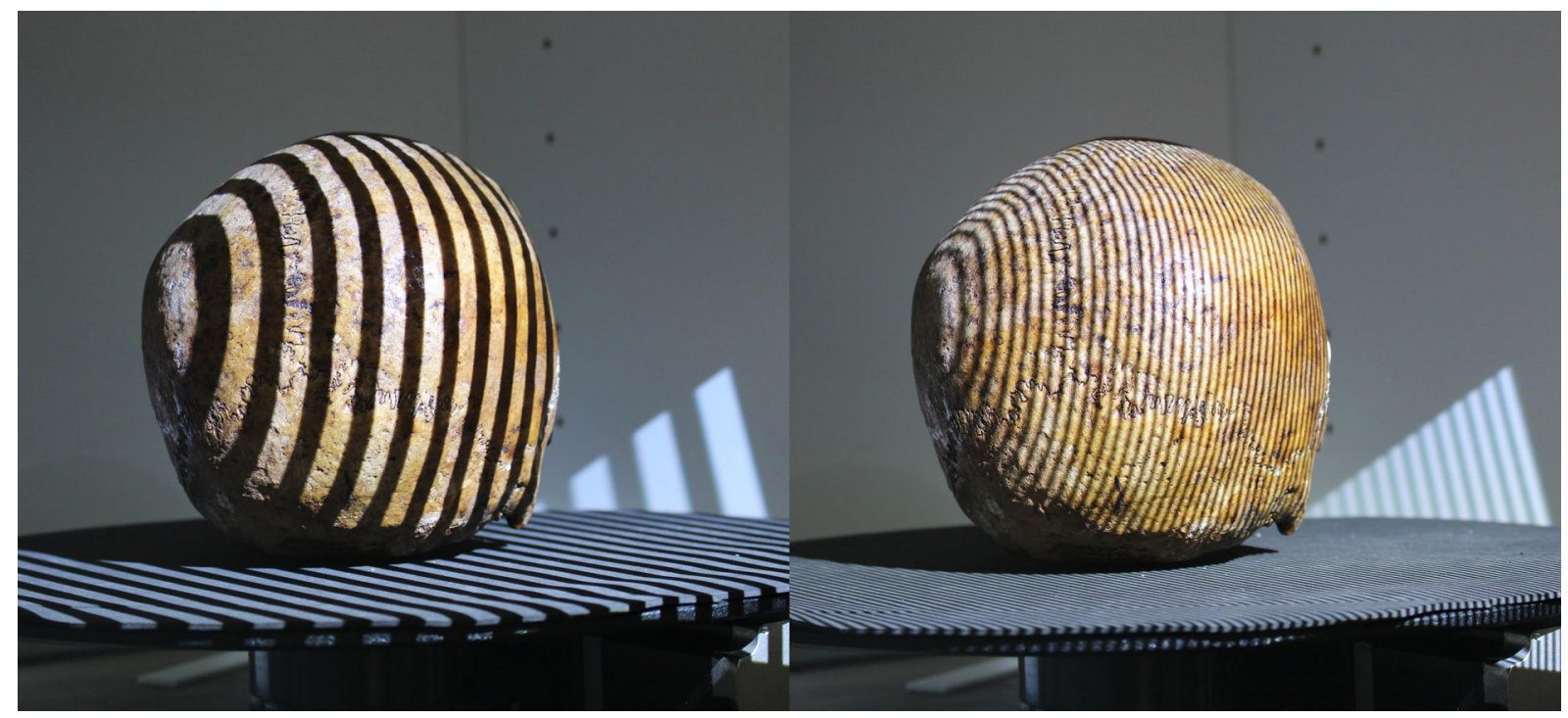

Fig. 3.22. Fringe projection.

Some structured light scanners allow the recording of colour texture. To do this, an additional image is captured for each scan without any pattern projected (Gorthi \& Rastogi 2010). White-light and infraredlight structured light scanners often enable colour acquisition for the texture while scanners using blue and red light usually do not record colour texture. The technique is already widely used in zoological studies (Botton-Divet et al. 2015; Fabre et al. 2016; Segal et al. 2016; Botton-Divet et al. 2017), excavations (McPherron et al. 2009), anthropology (Slizewski et al. 2010; Friess 2012; Maté-Gonzalez et al. 2017; Courtenay et al. 2018), paleontology (Johnston et al. 2013; Fabre et al. 2014; GuttierrezGarcia et al. 2015), etc., demonstrating its proven use in diverse fields of natural history (Mathys et al., 2013, 2014, 2015).

\subsubsection{Material and methods}

The HDI Advance (Fig. 3.23; https://www.polyga.com/hdi-3d-scanner/) is a structured light scanner using two cameras with an adjustable field of view. There are three predefined fields of view, of approximately $200 \mathrm{~mm}, 400 \mathrm{~mm}$ and $600 \mathrm{~mm}$. The 3Mp sensors have a width of approximately 2000 pixels. Therefore, 


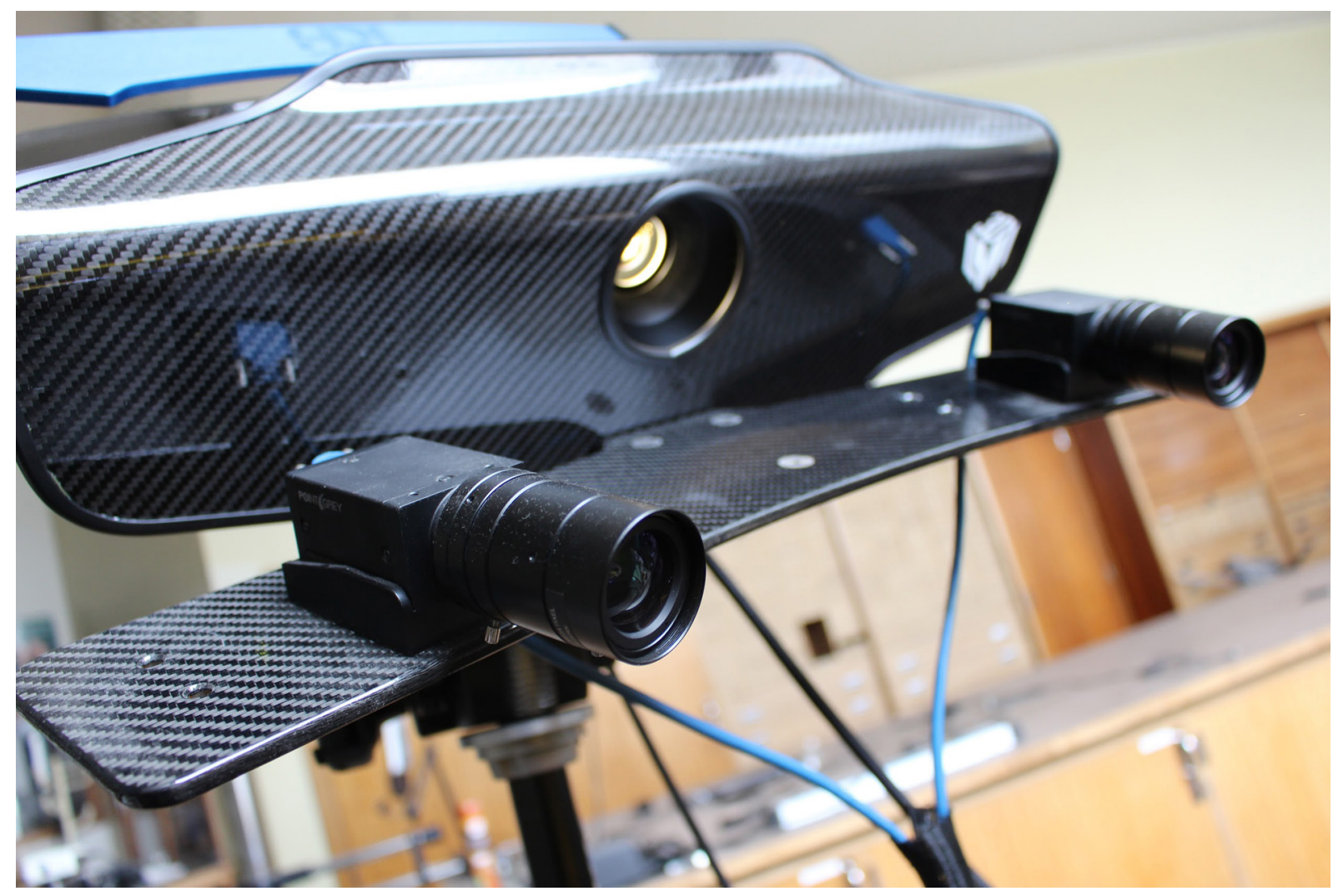

Fig. 3.23. The HDI white light scanner.

the $200 \mathrm{~mm}$ FOV equals in $0.1 \mathrm{~mm}$ Point to Point spacing (200 mm/2000). Generally, 20-30 points across a feature are needed to clearly define it. In terms of the size, it depends on the acceptable level of definition required. However it is not recommended to scan objects less than $45 \mathrm{~mm}$ in overall size because the obtained quality will not be high enough. If the level of detail is not that important and one is only looking for quantitative data, like surface to volume ratios, it is possible to scan smaller specimens (Kühsel et al. 2017). For each time the field of view is changed, the scanner has to be calibrated with a calibration board, which is a simple and very fast process (about a minute to complete the calibration).

The accuracy of the scanners depends on factors such as user experience, surface finish, calibration board accuracy, calibration technique and quality of overlapping registration data, to name a few of the main factors. A trained person can achieve about $0.25-0.1 \mathrm{~mm}$ resolution for the complete project.

The use of two cameras improves the precision as the scanner has two reference points. The scanner can be equipped with a monochrome- or a RGB camera. At RBINS the scanner is assembled with RGB cameras as a colour texture is very important. The acquisition takes a few minutes and is usually done with a turntable. But it is also possible to manually rotate the object or circle around it. In all cases the realignment is automatic, but can also be made manually if needed. This scanner is adapted to specimens with a diameter larger than $10 \mathrm{~cm}$.

A DSLR can be added as a third camera in order to improve the texture. It is controlled by the scanning software like the other cameras.

The MechScan (Fig. 3.24; http://www.mechscan.co.uk/) can be considered as the little brother of the HDI as it uses the same software, FlexScan3D (https://www.polyga.com/hdi-3d-scanner/flexscan3D/), 


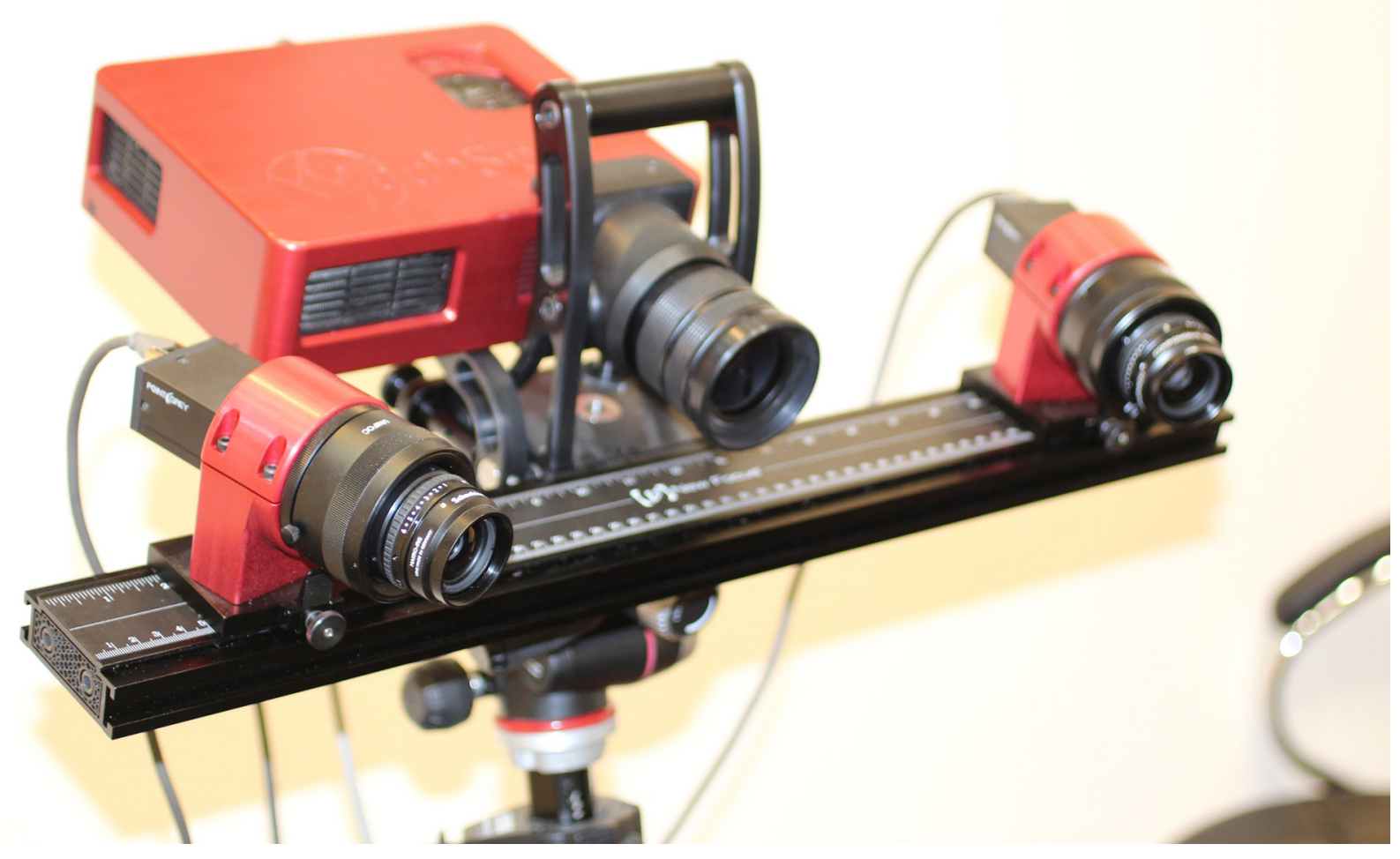

Fig. 3.24. The MechScan white light scanner.

although it is not a Polyga scanner but an independent scanner developed by Simon Stone of MechInnovation Limited in the UK. It differs from the HDI as it is dedicated to scan smaller objects $(2-10 \mathrm{~cm})$. Like the HDI it can also work with a DSLR camera commanded by the software and the same automated turntable.

Time of capture for a simple shape between 2 and $10 \mathrm{~cm}$ diameter (like a mollusk shell for example):

-5 mins for calibration

-2 mins 45 secs for a rotation of 8 scans in normal mode (usually advised to do 3 rotations)

$-40-60$ secs to combine scans

- 16-20 secs to align 2 combined scans

- Consider approximately 20 minutes in total (in normal mode)

\subsubsection{Collections}

The HDI (Figs 3.25-3.26) can be used for specimens larger than $10 \mathrm{~cm}$, while the MechScan (Figs 3.273.28 ) has an optimal object size of $2-10 \mathrm{~cm}$. Scanning larger objects with the latter is possible, although it is necessary to make more scans as the rotary table will not be used. In essence while scanning, one needs to see which parts are missing and alter the position of the object or move the scanner to capture missing parts. This means more scans are needed to capture the complete model and it will take more time to align, combine and finalize the model.

\subsubsection{References}

Bell T., Li B. \& Zhang S. 2016. Structured light techniques and applications. In: Webster J. (ed.) Wiley Encyclopedia of Electrical and Electronics Engineering: 1-24.

https://doi.org/10.1002/047134608X.W8298 


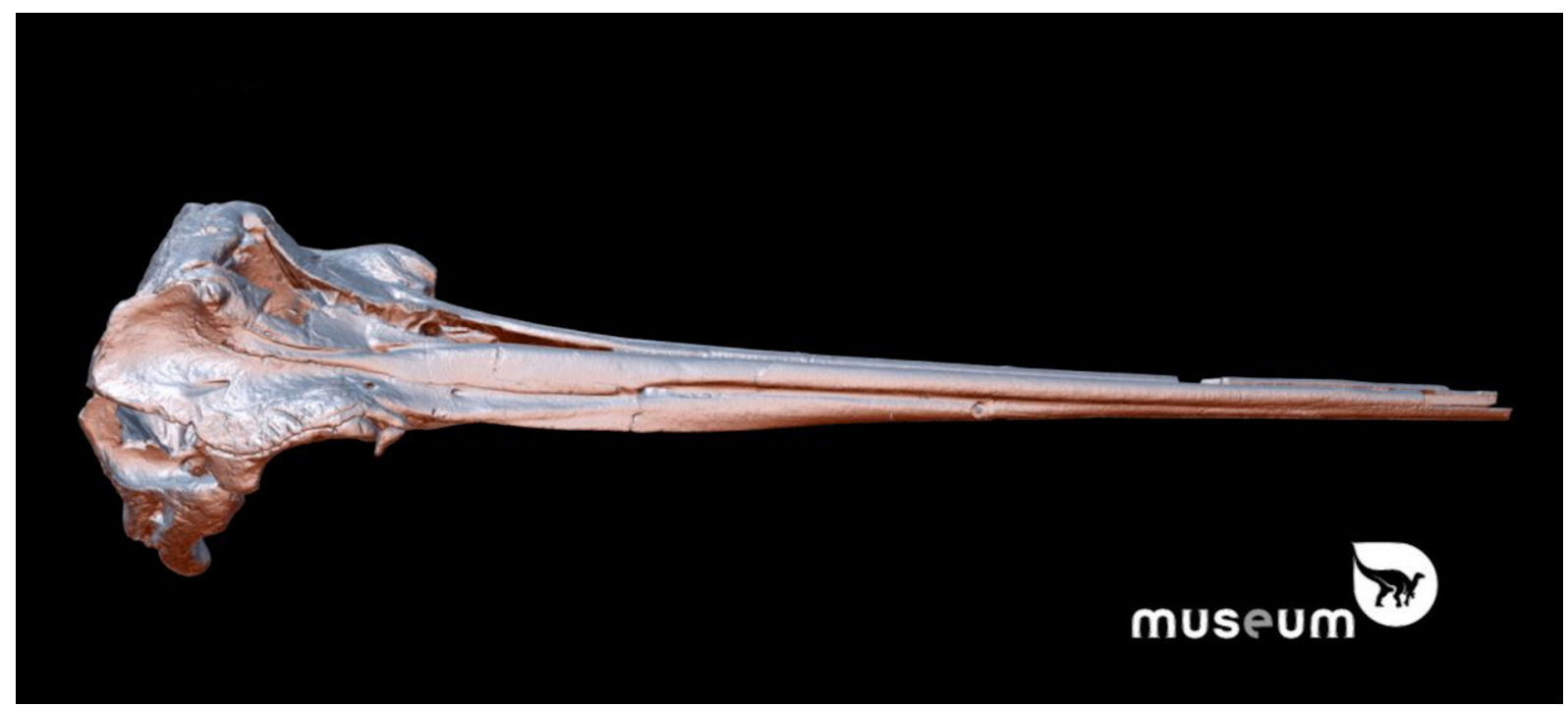

Fig. 3.25. Type specimen of Eurhinodelphis cocheteuxi, from the early to middle Miocene. Skull length $1074 \mathrm{~mm}$. Scanned with HDI. https://skfb.ly/6QQvU

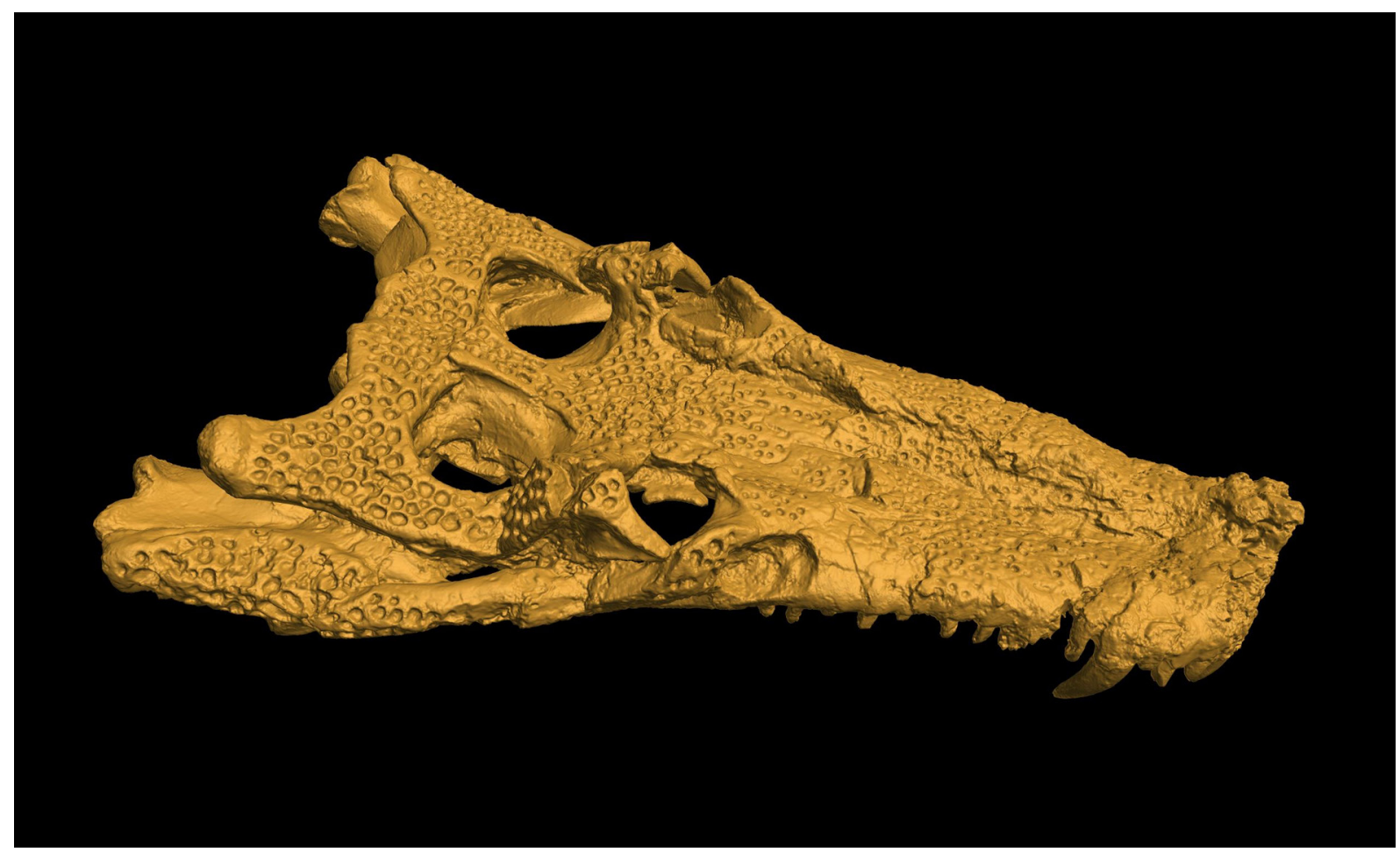

Fig. 3.26. Goniopholis skull. Scanned with the HDI.

https://sketchfab.com/models/c58694337a46449f9074b72fcb3c9cf5 


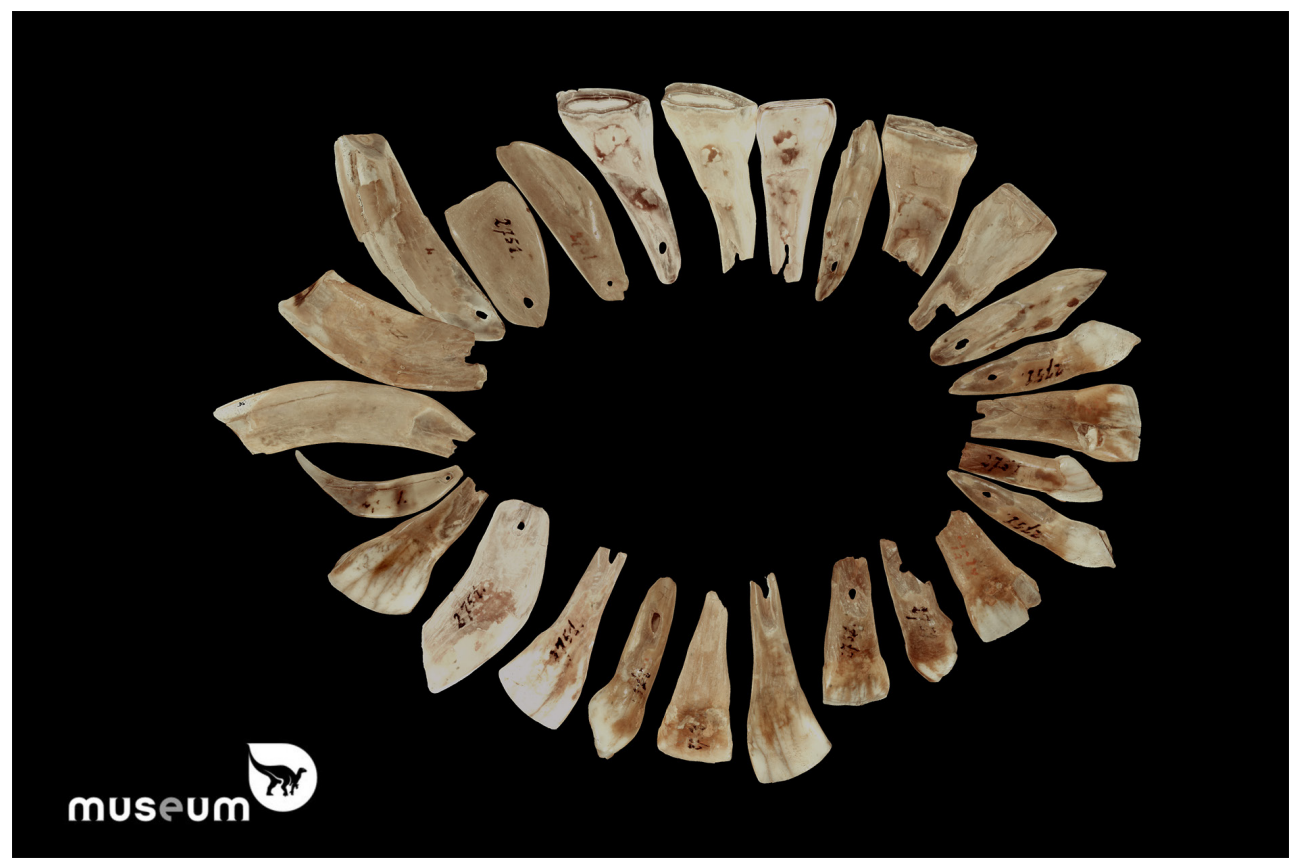

Fig. 3.27. Paleolithic horse-teeth necklace from Goyet, scanned with MechScan and reassemble https://sketchfab.com/models/b451e1803eb5461 fa8d685ea2a152ece

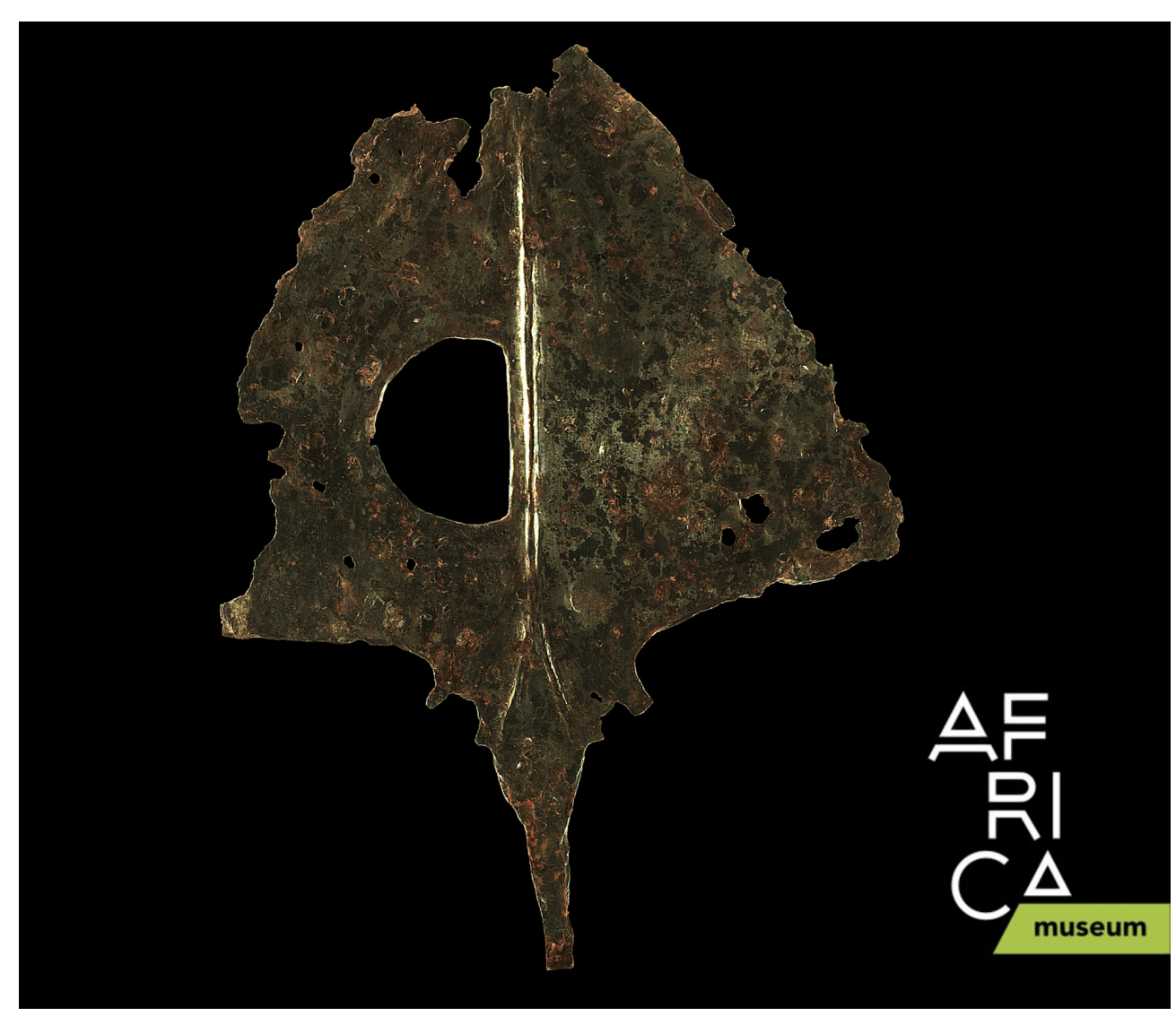

Fig. 3.28. $10^{\text {th }}$-century ceremonial axe from the necropolises of the Upemba Depression in Katanga (DRC), scanned with Mechscan https://sketchfab.com/models/8638588190bd4d3e88b857e7d94fc4cc 
Botton-Divet L., Cornette R., Houssaye A., Fabre A.-C. \& Herrel A. 2017. Swimming and running: a study of the convergence in long bone morphology among semi-aquatic mustelids (Carnivora: Mustelidae). Biological Journal of the Linnean Society 121 (1): 38-49. https://doi.org/10.1093/biolinnean/blw027

Botton-Divet L., Houssaye A., Herrel A., Fabre A.-C. \& Cornette R. 2015. Tools for quantitative form description; an evaluation of different software packages for semi-landmark analysis. PeerJ 3: e1417. https://doi.org/10.7717/peerj.1417

Chen F., Brown G.M. \& Song M. 2000. Overview of 3-D shape measurement using optical methods. Optical Engineering 39 (1). https://doi.org/10.1117/1.602438

Courtenay L.A., Maté-González M.Á., Aramendi J., Yravedra J., González-Aguilera D. \& DomínguezRodrigo M. 2018. Testing accuracy in 2D and 3D geometric morphometric methods for cut mark identification and classification. PeerJ 6: e5133. https://doi.org/10.7717/peerj.5133

Fabre A.-C., Bickford D., Segall M. \& Herrel A. 2016. The impact of diet, habitat use, and behaviour on head shape evolution in homalopsid snakes. Biological Journal of the Linnean Society 118 (3): 634-647. https://doi.org/10.1111/bij.12753

Fabre A.-C., Cornette R., Perrard A., Boyer D.M., Prasad G.V.R., Hooker J.J., \& Goswami A. 2014. A three-dimensional morphometric analysis of the locomotory ecology of Deccanolestes, a eutherian mammal from the Late Cretaceous of India. Journal of Vertebrate Paleontology 34 (1): 146-156.

https://doi.org/10.1080/02724634.2013.789437

Friess M. 2012. Scratching the surface? The use of surface scanning in physical and paleoanthropology. Journal of Anthropological Sciences 90: 7-31. https://doi.org/10.4436/jass.90004

Gorthi S.S. \& Rastogi P. 2010. Fringe projection techniques: whither we are? Optics and Lasers in Engineering 48 (2): 133-140. https://doi.org/10.1016/j.optlaseng.2009.09.001

Gutiérrez-García J.C., Gutiérrez-García T.A., Mosiño J.F., Vázquez-Domínguez E., Martínez-García A. \& Arroyo-Cabrales J. 2015. A novel application of the white light/fringe projection duo: recovering high precision three-dimensional images from fossils for the digital preservation of morphology. Palaeontologia Electronica 18.2.6T: 1-13. https://doi.org/10.26879/516

Johnston R.A., Barnes K.S., Lovell-Smith T. \& Price N. 2013. Use of a hand-held laser scanner in palaeontology: a 3D model of a plesiosaur fossil. Available from: https://www.polhemus.com/_assets/img/Use_of_a_Hand-held_Laser_Scanner_in_Palaeontology.pdf [accessed 19 Mar. 2020]

Kühsel S., Brückner A., Schmelzle S., Heethoff M. \& Blüthgen N. 2017. Surface area-volume ratios in insects. Insect Science 24: 829-841. https://doi.org/10.1111/1744-7917.12362

Maté-González M., Aramendi J., González-Aguilera D. \& Yravedra J. 2017. Statistical comparison between low-cost methods for 3D characterization of cut-marks on bones. Remote Sensing 9 (9): 873. https://doi.org/10.3390/rs9090873

Mathys, A., Brecko J. \& Semal P. 2014. Cost evaluation of 3D digitisation techniques. In: Ioannides M., Magnenat-Thalmann N., Fink E., Zarnic R., Yen A. \& Quak E. (eds) EUROMED 2014 Proceedings. MultiScience Ltd, Essex.

Mathys A., Brecko J., Van den Spiegel D. \& Semal P. 2015. 3D and challenging materials: guidelines for different 3D digitisation methods for museum collections with varying material optical properties. Proceedings of the $2^{\text {nd }}$ International Congress on Digital Heritage.

https://doi.org/10.1109/DigitalHeritage.2015.7413827 
Mathys A., Brecko J. \& Semal P. 2013. Comparing 3D digitising technologies: what are the differences? In: Addison A.C., De Luca L., Guidi G., Pescarin S. (eds) Digital Heritage International Congress: 201-204. CNRS, Marseilles. https://doi.org/10.1109/DigitalHeritage.2013.6743733

McPherron S.P., Gernat T. \& Hublin J.-J. 2009. Structured light scanning for high-resolution documentation of in-situ archaeological finds. Journal of Archaeological Science 36 (1): 19-24. https://doi.org/10.1016/j.jas.2008.06.028

Pavlidis G., Koutsoudis A., Arnaoutoglou F., Tsioukas V. \& Chamzas C. 2007. Methods for 3D digitisation of Cultural Heritage. Journal of Cultural Heritage 8 (1): 93-98.

https://doi.org/10.1016/j.culher.2006.10.007

Rieke-Zapp D. \& Royo S. 2017. Structured light 3D scanning. In: Bentkowska-Kafel A., MacDonald L. (eds) Digital Techniques for Documenting and Preserving Cultural Heritage: 247-251. Kalamazoo and Bradford: Arc Humanities Press. Amsterdam.

Sansoni G., Trebeschi M. \& Docchio F. 2009. State-of-the-art and applications of 3D imaging sensors in industry, cultural heritage, medicine, and criminal investigation. Sensors 9 (1): 568-601.

https://doi.org/10.3390/s90100568

Segall M., Cornette R., Fabre A.-C., Godoy-Diana R. \& Herrel A. 2016. Does aquatic foraging impact head shape evolution in snakes? Proceedings of the Royal Society B: Biological Sciences 283 (1837): 20161645. https://doi.org/10.1098/rspb.2016.1645

Slizewski A., Friess M. \& Semal P. 2010. Surface scanning of anthropological specimens: nominalactual comparison with low cost laser scanning and high-end fringe light projection surface scanning systems. Quartär 57: 179-187

Zachar J., Hornak M. \& Novakovic P. 2017. 3D digital recording of archaeological, architectural and artistic heritage. CONPRA series I. https://doi.org/10.4312/9789610603061 


\subsection{Laser Scanning}

Laser scanning is a non-contact technique to capture the surface of an object in 3D using a laser beam. Laser scanners can be divided in three categories based on their scanning principle: Triangulation laser scanner (short range), phase shift (medium range) and pulse-range (medium and long range). The accuracy of a laser scanner depends on the scanning principle (Feng 2012), the intensity of the reflected laser light (Fröhlich \& Mettenleiter 2004) and the model of the scanner. Most of modern laser scanners come now with a built-in camera to capture colour (Hess 2017).

Triangulation laser scanners have a short range and are used to digitise in 3D small or medium sized objects. The principle of a triangulation scanner is similar to the structured light scanner, but in this case, it is a laser beam (shaped as a line) that is projected onto the surface of the object. The laser stripe is reflected by the surface and captured by an optical sensor (Shan \& Toth 2018, Hess 2017). The distance between the source of the laser and the optical sensor is known as is the angle of emission of the laser. Accuracy of a triangulation scanner is variable according to the brand/model of scanner used, but it can be up to a few micrometres (Fröhlich \& Mettenleiter 2004).

Triangulation scanners come in different forms; a table-top with turntable or a handheld scan-arm are the most common forms.

Phase-shift scanners are also known as phase comparison or continuous wave scanners and are used for medium range applications (indoor or outdoor). They are mostly used in ground applications and are well represented among terrestrial laser scanners (TLS).

Phase-shift scanners emit a continuous laser wave (Randall 2013) and measure the phase difference between the transmitted and the received laser wave (Feng 2012). They have a very high rate, resulting in a high accuracy of a few mm (Shan \& Toth 2018, Fröhlich \& Mettenleiter 2004). Their maximum range used to be up to $100 \mathrm{~m}$, but recent tools can go up to several hundred meters (Shan \& Toth 2018).

The pulse laser scanner, also known as Time of Flight (ToF) or ranging scanners, are used for middle range and long-range application for both TLS and Airborne Laser Scanning (Fröhlich \& Mettenleiter 2004; Feng 2012; Shan \& Toth 2018). Light detection and ranging (LiDAR) is a pulse scanner, this term is mostly used for airborne systems (Historic England 2018).

ToF uses a pulsed laser beam to measure the time between the emitted pulse and its return to the receiver after being reflected by the target surface by means of known light velocity (Thiel \& Wehr 2004). ToF have a maximum range of a few-hundred meters to several kilometers, depending on the model (Fröhlich \& Mettenleiter 2004; Ebrahim 2015; Feng 2012; Randall 2013; Shan \& Toth 2018). They have a lower rate than phase-shift scanners, resulting in a slightly lower accuracy (Fröhlich \& Mettenleiter 2004).

In the past, pulse scanners were used where a longer range was needed and phase scanners were used to obtain better accuracy, but nowadays the two techniques are convergent (San José Alonso et al. 2011). Indeed, pulse scanner acquisition rates have increased while phase scanners can be used in longer range (although not as long as some ToF).

Most pulse and phase-shift scanners do not require external power supplies allowing for high mobility (Historic England 2018).

\subsubsection{Material and methods}

The NextEngine (Fig. 3.29) is a multi-stripe laser triangulation scanner. It is auto-calibrated and can produce a colour texture. It has three modes of scanning (wide, standard and macro; Fig. 3.30) allowing for variable precision. It also captures colour texture. The scanner does not work well with shiny reflective objects, as is the case for most laser scanners. Generally, in industrial scanning, a coating will be sprayed 


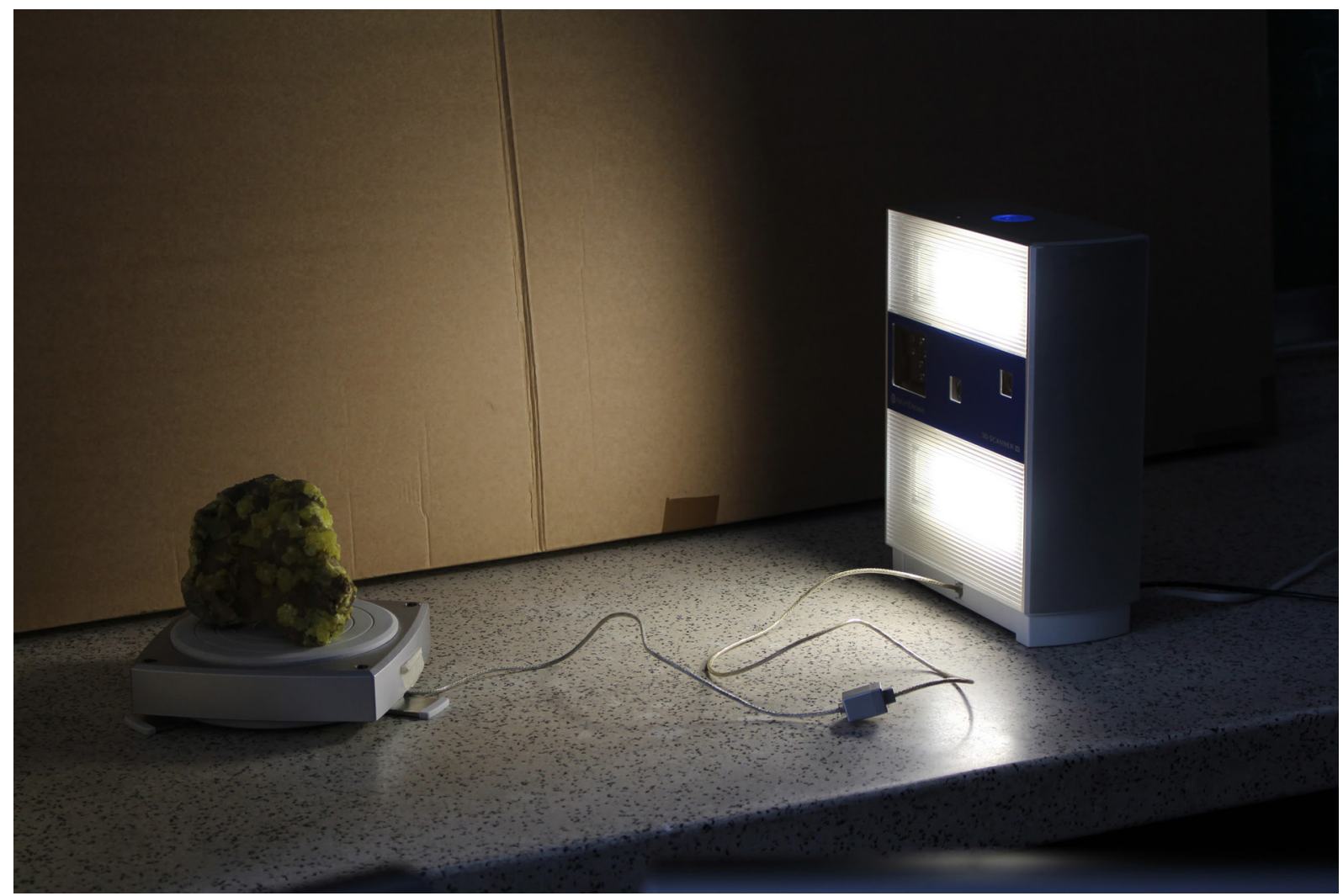

Fig. 3.29. NextEngine with the rotary table holding a geological sample. The cardboard sheet behind is placed to avoid reflection of the bright and shiny glazed white tiles on the wall. The NextEngine also comes with a sample holder to scan small samples.

on the reflective object, but this is often not possible for a museum specimen. All in all, it is slow to capture a full specimen, but on the other hand it is a benefit that the scanning process is automated.

The NextEngine is a relatively low-cost laser scanner, good for occasional digitisation. It was the first $3 \mathrm{D}$ equipment acquired at RBINS and now is seldom used, as it has been replaced by techniques that are more efficient or allow a better structure for visualisation like SfM and Structured Light Scanning.

Comparison of the NextEngine with other techniques has shown that NextEngine, although less precise than $\mu \mathrm{CT}$ or state-of-the-art structured light scanner, gives good results for the price (Slizewski et al. 2010; Polo \& Felicísimo 2012; Mathys et al 2014).
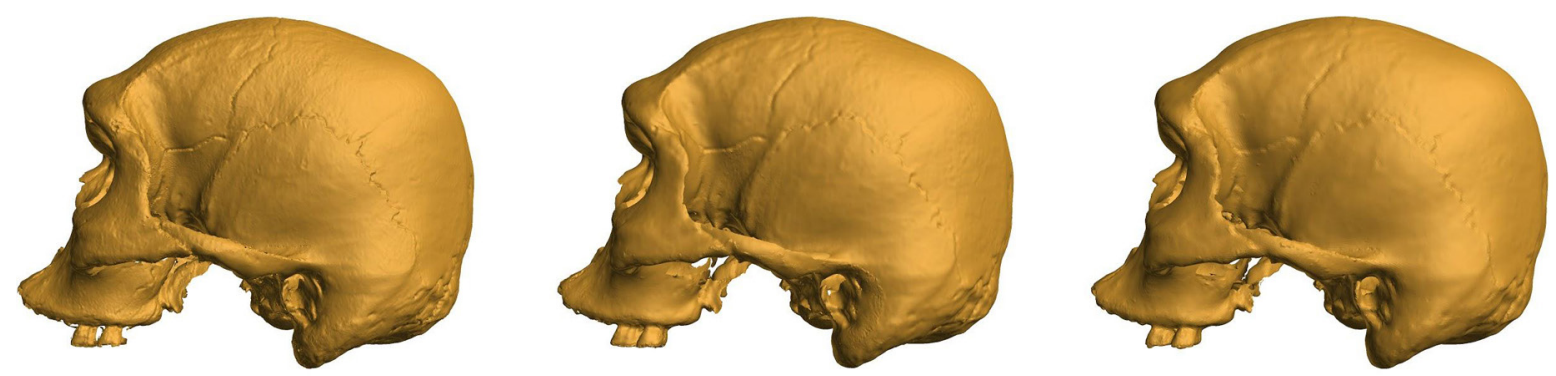

Fig. 3.30. Human skull digitised with NextEngine. On the left in macro mode, in the middle in standard mode and on the right with wide mode. 
Typical time of scanning with the NextEngine for a simple middle-sized object: set up and preparation of equipment requires roughly 15 mins.

Acquisition time: consider approximately $40 \mathrm{mins}$ for a single rotation to obtain decent quality; a minimum of two rotations are needed for a full simple object (50000 processed points/sec, 2 minutes per scan of each facet).

Post-processing: the time depends on the aims and the computer, but it can be quite long. To finalise the model, a lot of post-processing is needed, especially when using wide settings. Also, in wide range, the texture produced tends to be blueish. Saving the data in the software (ScanStudio, $\mathrm{http} / /$ /www.nextengine.com/products/scanstudio-hd/specs/overview) is very slow and it is recommended to save the project between every step because the software often crashes.

\subsubsection{Collections}

When used for objects between 6-25 cm, the turntable is ideal (Figs 3.30-3.32), but it can be used for larger structures without using the turntable. However, this requires more post-processing time. This laser scanner is very practical for occasional digitisation of objects where SfM does not work, for instance with plaster objects. If time is not an issue and the mesh quality is more important than the texture, than the NextEngine can be a good solution. It is unadvised for objects where good texture is important.

\subsubsection{References}

Ebrahim M.A. 2015. "3D Laser Scanners' Techniques Overview", International Journal of Science and Research (IJSR) 4 (10): 323-331. Available from:

https://www.ijsr.net/search_index_results_paperid.php?id=SUB158346 [accessed 19 Mar. 2020]

Historic England. 2018. 3D Laser Scanning for Heritage: Advice and Guidance on the Use of Laser Scanning in Archaeology and Architecture. Historic England, Swindon.

Feng Q. 2012. Practical application of 3D laser scanning techniques to underground projects, phase 2-3 a part of ISRM-Swedish national task: "A survey of 3D laser scanning techniques for application to rock mechanics". BeFo Report 114. Stockholm, Sweden.
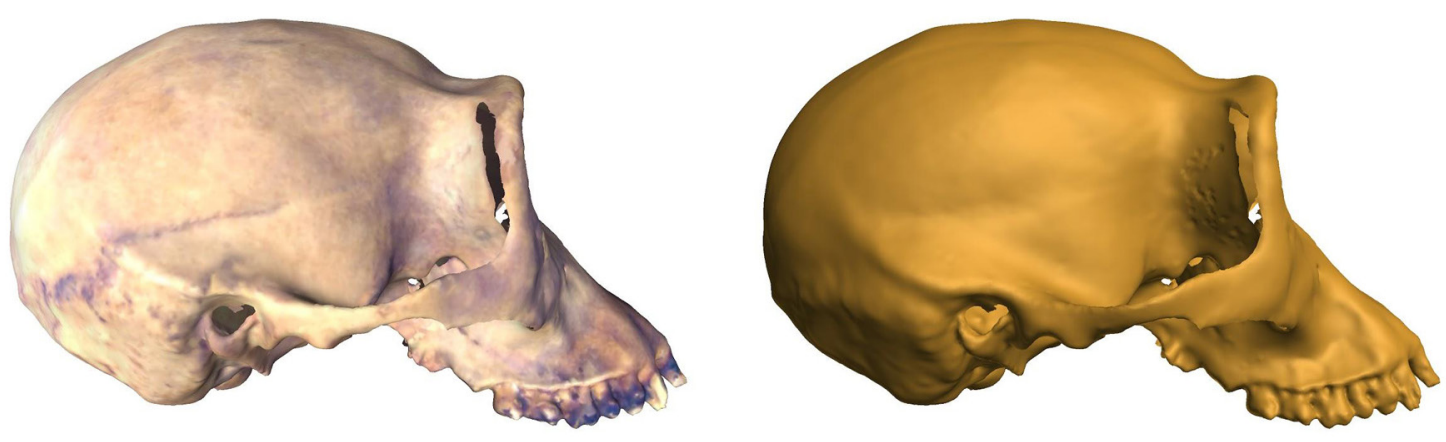

Fig. 3.31. Skull of Pan paniscus (RMCA) scanned with NextEngine. Left: the mesh with texture; right: the mesh without texture. https://sketchfab.com/models/38295c2ee9dd428f93134d0e97ffe851 


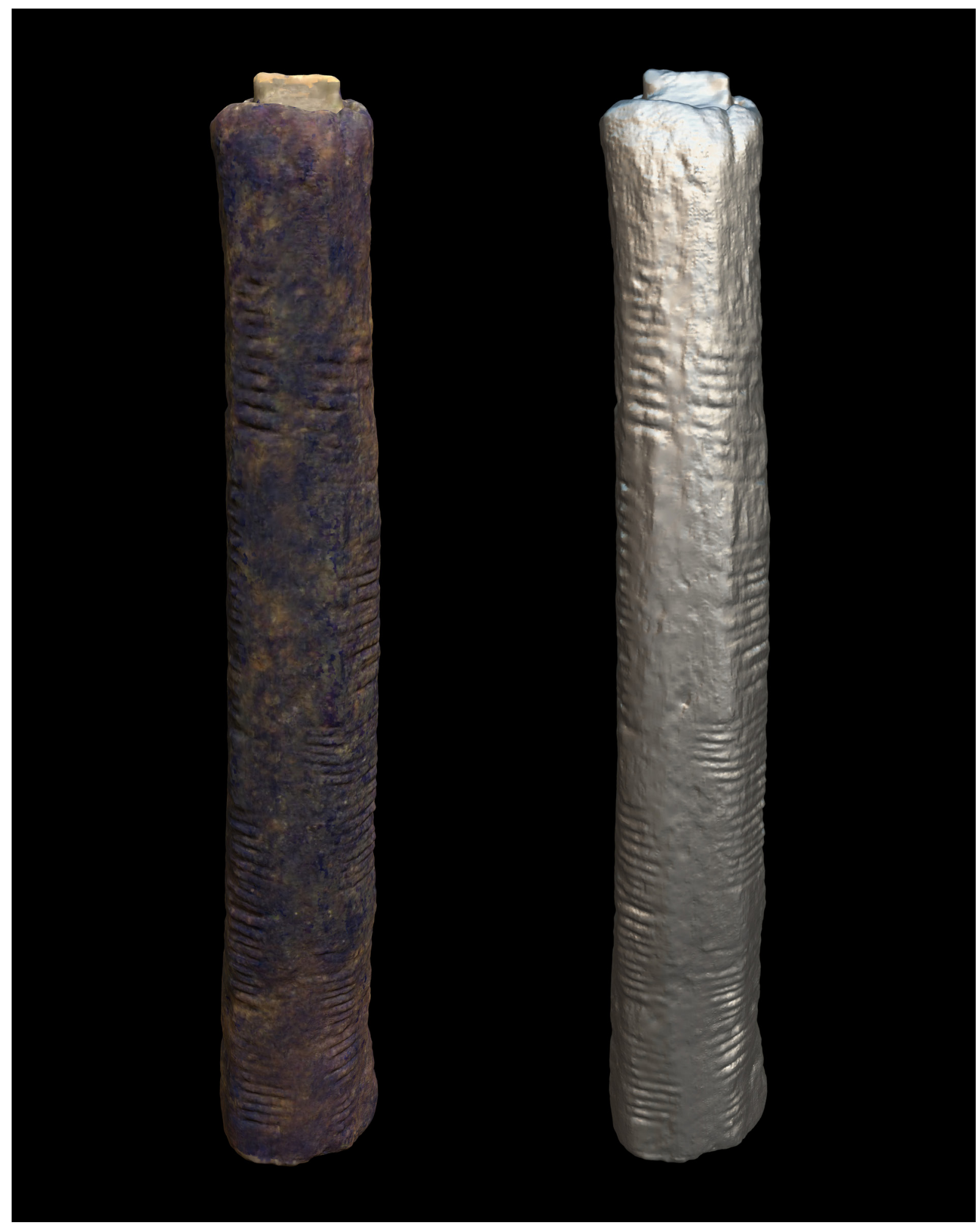

Fig. 3.32. Ishango rod digitised with NextEngine.

https://sketchfab.com/models/555f0a85ca224ab88f3712272d877ce7 
Fröhlich C. \& Mettenleiter M. 2004. Terrestrial laser scanning - new perspectives in 3D surveying. ISPRS - International Archives of the Photogrammetry, Remote Sensing and Spatial Information Sciences XXXVI-8/W2: 7-13.

Hess M. 2017. 3D Laser Scanning. In: Bentkowska A. \& MacDonald L. (eds) Digital Techniques for Documenting and Preserving Cultural Heritage: 199-206. Kalamazoo and Bradford: Arc Humanities Press, Amsterdam.

Mathys A., Brecko J. \& Semal P. 2014. Cost evaluation of 3D digitisation techniques. In: Ioannides M., Magnenat-Thalmann N., Fink E., Zarnic R., Yen A. \& Quak E. (eds) EUROMED 2014 Proceedings. MultiScience Ltd, Essex.

Polo M.-E. \& Felicísimo Á. M. 2012. Analysis of uncertainty and repeatability of a low-cost 3D laser scanner. Sensors 12 (7): 9046-9054. https://doi.org/10.3390/s120709046

Randall T. 2013. Client guide to 3D scanning and data capture. Building information modelling (BIM) task group. Available from

http://bim-level2.org/globalassets/pdfs/clients-guide-to-3d-scanning-and-data-capture.pdf [accessed 30 Aug. 2018]

San José Alonso J.I., Martínez-Rubio J., Fernandez J. \& Garcia Fernandez J. 2011. Comparing timeof and phase-shift the survey of the Royal Pantheon in the Basilica of San Isidoro (LEÓN). ISPRS - International Archives of the Photogrammetry, Remote Sensing and Spatial Information Sciences XXXVIII-5/W16: 377-385. https://doi.org/10.5194/isprsarchives-XXXVIII-5-W16-377-2011

Shan J. \& Toth C.K. 2008. Topographic Laser Ranging and Scanning: Principles and Processing, Second Edition. CRC Press, Boca Raton. https://doi.org/10.1201/9781420051438

Slizewski A., Friess M. \& Semal P. 2010. Surface scanning of anthropological specimens: nominalactual comparison with low-cost laser scanning and high-end fringe light projection surface scanning systems. Quartär 57: 179-187.

Thiel K.H. \& Wehr A. 2004. Performance capabilities of laser scanners - an overview \& measurement principle analysis. Proceedings of ISPRS International Archives of the Photogrammetry, Remote Sensing and Spatial Information Sciences XXXVI-8/WG2: 14-18. 


\subsection{Infrared Depth Sensor}

The infrared depth sensor family (Kinect based), also known as an RGB-D sensor, was originally a motion sensor device used for the Microsoft Xbox video games. There are currently several different brands of sensors which are used for 3D scanning. These sensors are very fast (several million points per second) and data transfer happens via a USB 2.0 connection to a laptop or tablet to gain flexibility in scanning. If large objects are scanned it is best to transfer the data to a powerful workstation. The need for an onsite power supply depends on the battery lifetime of the computer. The accuracy is approximately $1 \mathrm{~mm} \pm 0.3$ (Carey et al. 2017); the technique is therefore intended to record large structures (Fig. 3.33). This relatively recent technology is promising for large objects or for field recording, but also for poor lighting conditions (if texture is not important) as it can capture 3D in complete darkness (Mathys et al. 2013).

RGB-D sensors have an infrared projector and two cameras: one for infrared and one for colour. The first generation of infrared sensors (Kinect v1, Carmine, Xtion Pro, RealSense, Structure Sensor) are low-cost versions of a structured light scanner (Darwish et al. 2017; Villena-Martinez et al. 2017). Indeed, they project a pattern in infrared captured by the IR camera and the 3D data is calculated using the triangulation principle (Alhwarin et al. 2014; Zennaro et al. 2015). Second generation sensors (Kinect v2, Senz3D) use the time of flight principle to generate 3D (Lachat et al. 2015; Zennaro et al. 2015; Choe et al. 2016; Pöhlmann et al. 2016; Villena-Martinez et al. 2017). Numerous tests between the sensors of different generations (Zennaro et al. 2015), different manufacturers (Kersten et al. 2016; Carfagni et al. 2017) and even between units of the same model (Boehm 2014) have been carried out. The main conclusion of recent tests is that all of them perform well in repeatability of measurements (Boehm 2014; Zennaro et al. 2015; Kersten et al. 2016; Carfagni et al. 2017). Other outcomes are that the Kinect v2 performs better than the v1, and is more robust in different light conditions (Zennaro et al.

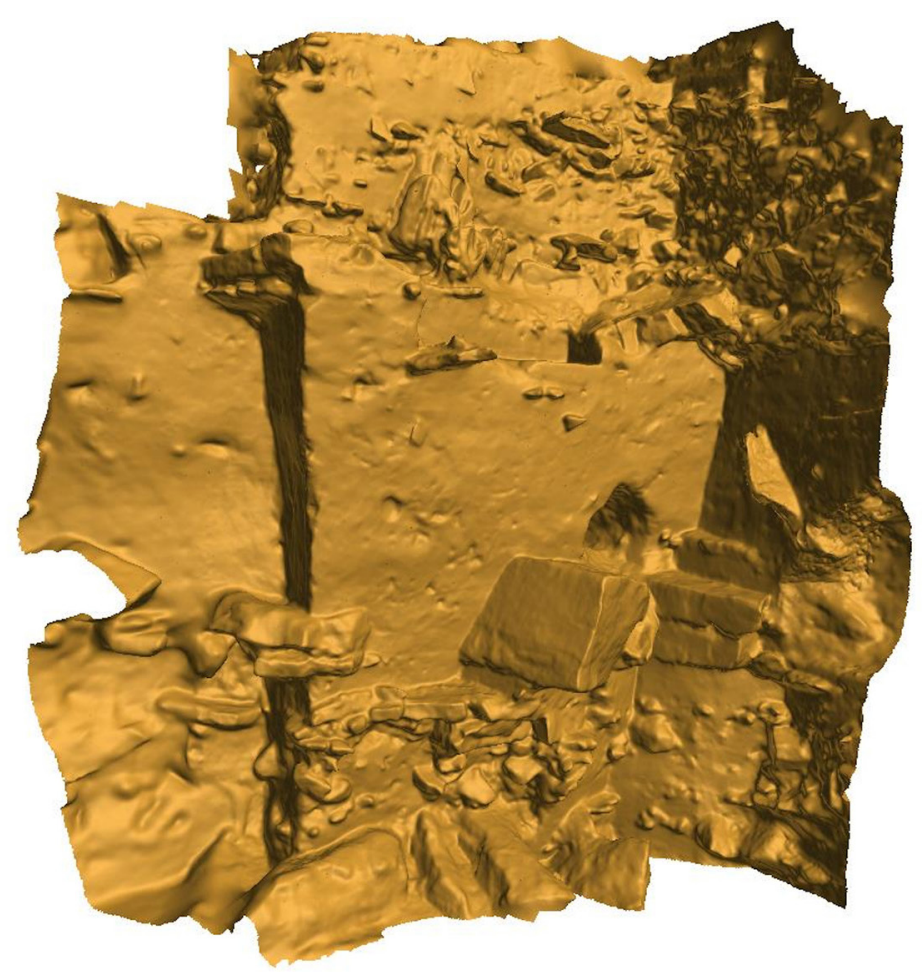

Fig. 3.33. Scan of a part of the excavation at Scladina cave (Belgium) made with the Gotcha. The part of the site pictured measures approximately $2 \times 2 \mathrm{~m}$. 
2015). Both the Structure Sensor and the RealSense SR300 perform better than other sensors (Kinect v2, Carmine 1.09) in the combination of capturing the overall shape and the ability to keep measurements to scale (Kersten et al. 2016; Carfagni et al. 2017). However, it muste be pointed out that intra-manufacturer comparisons have showed that sometimes a severe difference in scanning performance exists (Boehm 2014). Therefore, Boehm (2014) recommends acquiring different sensors, testing them and only using the most accurate ones.

At the moment the RGB-D sensors at RBINS are not used often due to the availability of better, more accurate techniques in the digitisation centre. The RGB-D sensor is ideal for producing 3D models of large objects in a fast way (no time for SfM), when the lower precision is sufficient relative to the object size and if there is no budget for a better quality scanner. The resulting 3D models can be used to produce high quality 3D models like the 3D printed model of the Mammoth of Lier produced by Materialise based on 3D models of RBINS (https://www.materialise.com/en/cases/3d-printing-meetsprehistoric-preservation-materialise-prints-life-size-mammoth-skeleton) However, it has proven to be an interesting documentation aid in field work like like excavations, when immediate results are necessary (Mathys et al. 2013).

\subsubsection{Material and methods}

The device features an RGB camera, a dedicated IR camera and an infrared laser projector for depth sensing (Fig. 3.34). Various software exists for motion sensing, among which: Scenect,

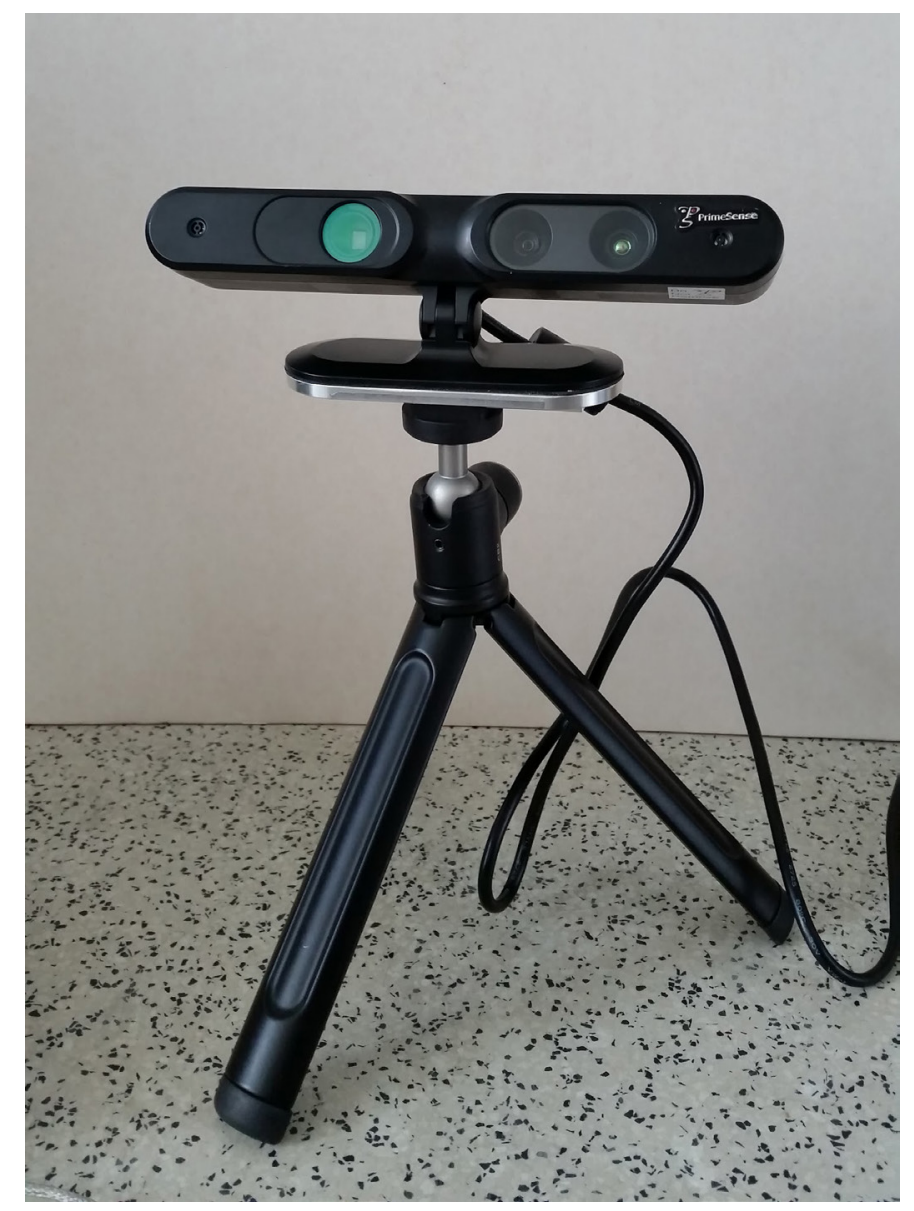

Fig. 3.34. The Primesense infrared depth sensor of the Gotcha with a tripod, allowing it to stand or be used handheld while scanning. 
Skanect, ReconstructMe, Gotcha and ItSeez3D. Of all the software tested, the Artec Studio (https://www.artec3d.com/3d-software/artec-studio) offered a considerable advantage as it can realign based upon shape and texture. It was also more user friendly than the software of the Gotcha (http://www.4ddynamics.com/\#portfolio), which was used to scan both the Elephant of RMCA(Fig. 3.35) and the dismantled Mammoth (Fig. 3.36) of RBINS before mounting. Regarding the Gotcha software, it worked well if only few scans required combining. Also, the black background of the software, which in early versions could not be changed, made it difficult to align dark objects like the fossil bones of a mammoth. Once a very large object like an elephant is scanned, aligning also becomes difficult, possibly due to changes in temperature, light conditions, or other parameters which can change during a scan and result in aberrations between different scans.

Considering free software (for educational purposes), the most stable ones tested were ReconstructMe (http://reconstructme.net/) and Skanect (http://skanect.occipital.com/). Looking at recent models hosted on Sketchfab, the ItSeez3D (https://itseez3d.com/) software looks quite good in captured texture and detail.

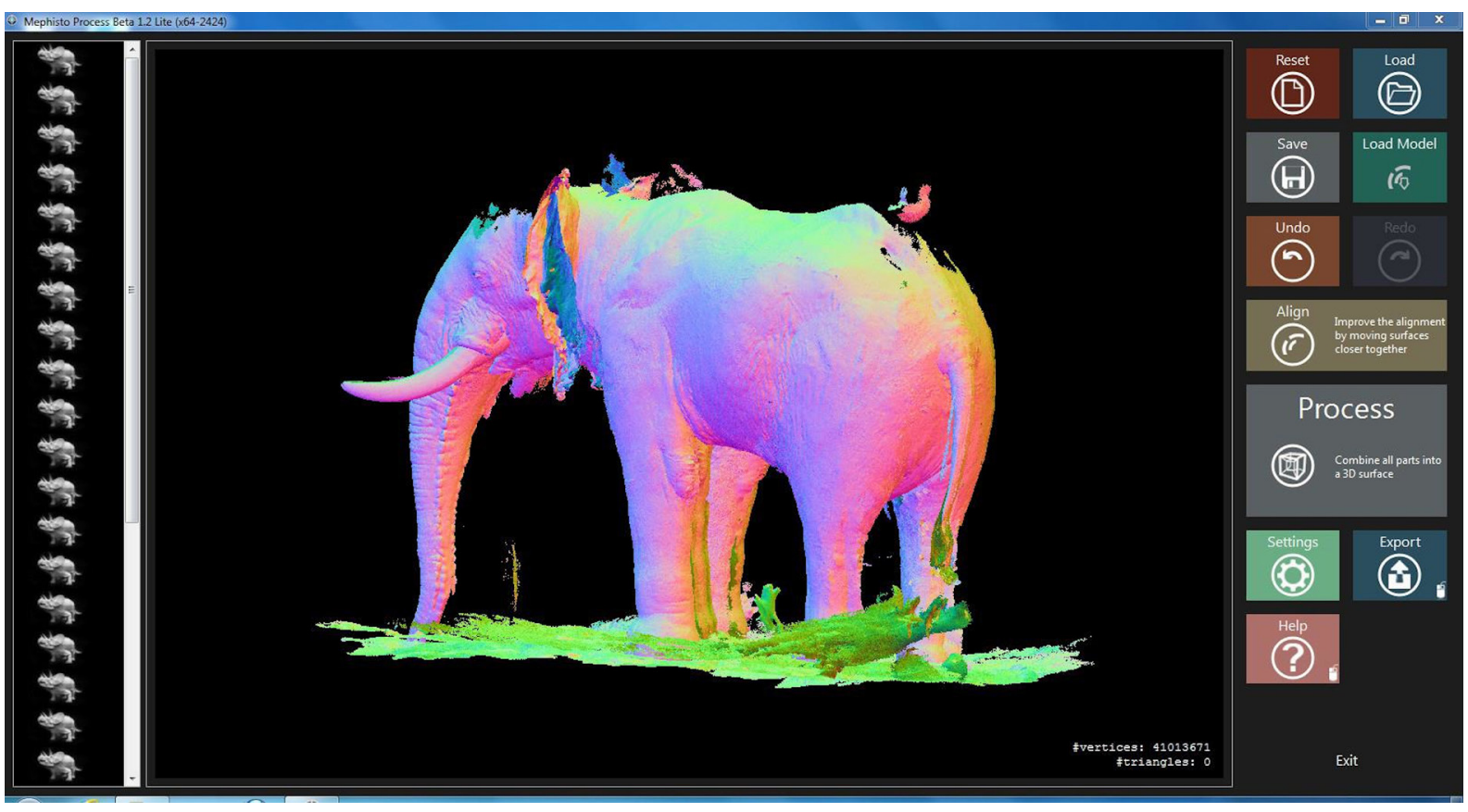

Fig. 3.35. Screen capture of the Mephisto scanning software while recombining scans of a stuffed African elephant produced with the Gotcha infrared sensor.

\subsubsection{Collections}

This technology can be used to scan large specimens like the complete skeleton of a mammoth (completely dismantled skeleton, scanned bone per bone at RBINS; Fig. 3.36), a Moai (Easter Island statue, scanned in $1 \mathrm{hr}$ at RMAH), an elephant (scanned in $2 \mathrm{hrs} 30$ mins at RMCA; Fig. 3.35) or an excavation site (Figs $3.33,3.37$ ). The moai was scanned with both the Gotcha and the Xtion-skanect package. The massive size of the Moai meant the technique worked really well, while with the mammoth skeleton was a bit more challenging for the thin bones like the ribs. As these are quite narrow they are difficult to scan. But these can be scanned by placing it on a larger structure, like a table, and scan the two together. The support structure can be cut in the digital scan afterwards. In general, the point clouds obtained with the Gotcha are good, but the software has trouble processing thin parts and creates holes in the mesh for 


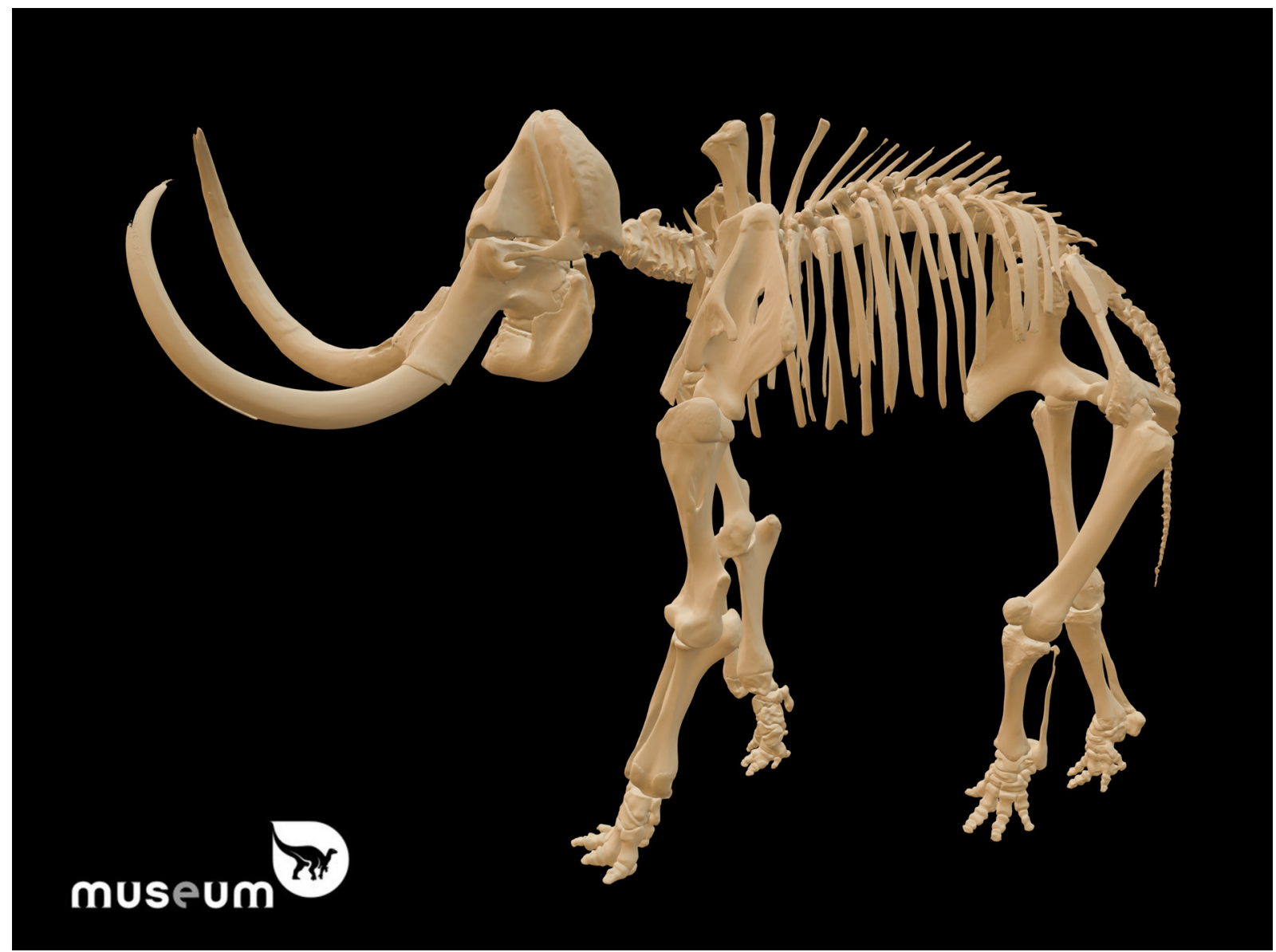

Fig. 3.36. 3D model of the mammoth on display at the RBINS permanent exhibition. The bones were scanned one by one at the moment the skeleton was disassembled to move it to another exhibition spot. The Gotcha infrared depth sensor was used and the different 3D models were virtually reassembled in lhpFusionBox (ULB, Brussels). https://sketchfab.com/models/2d25256368a44a0fb98d0418ac500d47

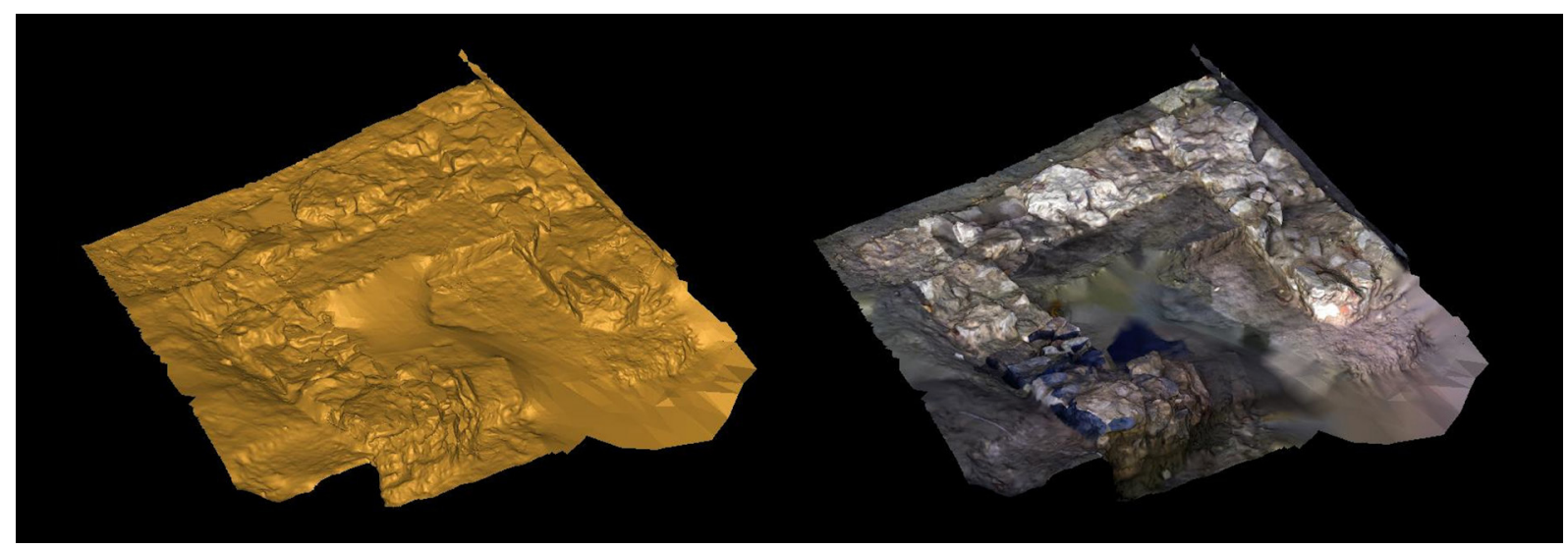

Fig. 3.37. Excavation site scanned with the Gotcha infrared sensor. On the left is the site without texture, on the right with texture. The excavation site pictured measures approximately $4 \times 4 \mathrm{~m}$. 
those parts. Recently, Das et al. (2017) published a paper on the scanning of Tyrannosaurus rex fossils using a kinect sensor.

\subsubsection{References}

AlhwarinF., Ferrein A. \& SchollI.2014. IR Stereo Kinect: Improving depth images by combining structured light with IR stereo. In: Pham D.N., Park S.B. (eds) Lecture Notes in Computer Science, Vol. 8862: PRICAI 2014: Trends in Artificial Intelligence. Springer, Cham. https://doi.org/10.1007/978-3-319-13560-1_33

Boehm J. 2014. Accuracy Investigation for structured-light based consumer 3D sensors. Photogrammetrie - Fernerkundung - Geoinformation 2014 (2): 117-27. https://doi.org/10.1127/1432-8364/2014/0214

Carey N., Werfel J. \& Nagpal R. 2017. Fast, accurate, small-scale 3D scene capture using a low-cost depth sensor. IEEE Winter Conference on Applications of Computer Vision (WACV): 1268-1276. https://doi.org/10.1109/WACV.2017.146

Carfagni M, Furferi R., Governi L., Servi M., Uccheddu F. \& Volpe Y. 2017. On the performance of the Intel SR300 Depth Camera: metrological and critical characterization. IEEE Sensors Journal 17 (14): 4508-4519. https://doi.org/10.1109/JSEN.2017.2703829

Choe G., Park J., Tai Y.W. \& Kweon I.S. 2016. Refining geometry from depth sensors using IR Shading Images. International Journal of Computer Vision 122: 1-16.

https://doi.org/10.1007/s11263-016-0937-y

Darwish W., Tang S., Li W., \& Chen W. 2017. A new calibration method for commercial RGB-D sensors. Sensors 17 (6): 1204. https://doi.org/10.3390/s17061204

Das A.J., Murmann D.C., Cohrn K. \& Raskar R. 2017. A method for rapid 3D scanning and replication of large paleontological specimens. PLoS ONE 12 (7): e0179264.

https://doi.org/10.1371/journal.pone.0179264

Kersten T.P., Przybilla H.-J., Lindstaedt M., Tschirschwitz F. \& Misgaiski-Hass M. 2016. Comparative geometrical investigations of hand-held scanning systems. ISPRS - International Archives of the Photogrammetry, Remote Sensing and Spatial Information Sciences XLI-B5: 507-514. https://doi.org/10.5194/isprsarchives-XLI-B5-507-2016

Lachat E., Macher H., Mittet M.A., Landes T. \& Grussenmeyer P. 2015. First experiences with Kinect V2 Sensor for close range 3D modelling. ISPRS - International Archives of the Photogrammetry, Remote Sensing and Spatial Information Sciences XL-5/W4: 93-100.

https://doi.org/10.5194/isprsarchives-XL-5-W4-93-2015

Mathys A., Brecko J., Di Modica K., Abrams G., Bonjean D. \& Semal P. 2013. Agora 3D. Low-cost 3D imaging: a first look for field archaeology. Notae Praehistoricae 33/2013: 33-42.

Pöhlmann S.T.L., Harkness E.F., Taylor C.J. \& Astley S.M. 2016. Evaluation of Kinect 3D Sensor for healthcare imaging. Journal of Medical and Biological Engineering. 36 (6): 857-870.

https://doi.org/10.1007/s40846-016-0184-2

Villena-Martínez V., Fuster-Guilló A., Azorín-López J., Saval-Calvo M., Mora-Pascual J., GarciaRodriguez J. \& Garcia-Garcia A. 2017. A Quantitative comparison of calibration methods for RGB-D sensors using different technologies. Sensors 17 (2): 243. https://doi.org/10.3390/s17020243

Zennaro S., Munaro M., Milani S., Zanuttigh P., Bernardi A., Ghidoni S. \& Menegatti E. 2015. Performance evaluation of the $1^{\text {st }}$ and $2^{\text {nd }}$ generation Kinect for multimedia applications. IEEE International Conference on Multimedia and Expo (ICME). https://doi.org/10.1109/icme.2015.7177380 


\section{Multispectral}

Multispectral imaging (MSI) consists of acquiring images over several specific bands of the light spectrum (ultraviolet, visible, near-infrared). Multispectral imaging can be combined with some of the previous techniques described in the handbook in order to capture extra information. In some cases, just one or two spectral bands will be sufficient, in others 10 or 16 are needed.

Multispectral imaging has already been used for a long time in astronomy, remote sensing, medicine, painting analysis, etc. It can be used for different applications to enhance details or to reveal details invisible in white light, to acquire precise colour information or to have composition information.

One of the main applications of spectral imaging for natural history collections is associated to the ultraviolet (UV) fluorescence of the specimens. As a matter of fact, many species display a specific pattern in fluorescence while exposed to UV light. It is the case for arthropods (Figs 4.1-4.2; Hennen 2012; Pearson 2013), diplopods (Fig. 4.3), fishes (Siebeck et al. 2010), amphibians (Taboada et al., 2017; Nowogrodzki, 2017; Thompson et al., 2017; Gouette et al., 2019). reptiles (Gruber \& Sparks 2015; Martin et al. 2013; Prötzel et al. 2018), corals (Alieva et al. 2008; Salih et al. 2000), Mammals (Kohler et al., 2019) and many others. UV patterns can be involved in species recognition (Ze-Lin et al. 2012; Brecko et al. 2016) and sexual selection (Brunton 1998), but are also dependent of environmental factors (Pechacek et al., 2019; Stella et al., 2018). UV fluorescence can also enhance the visualisation of fungus infestation (Fig. 4.4), restorations and treatments (Fig. 4.5).

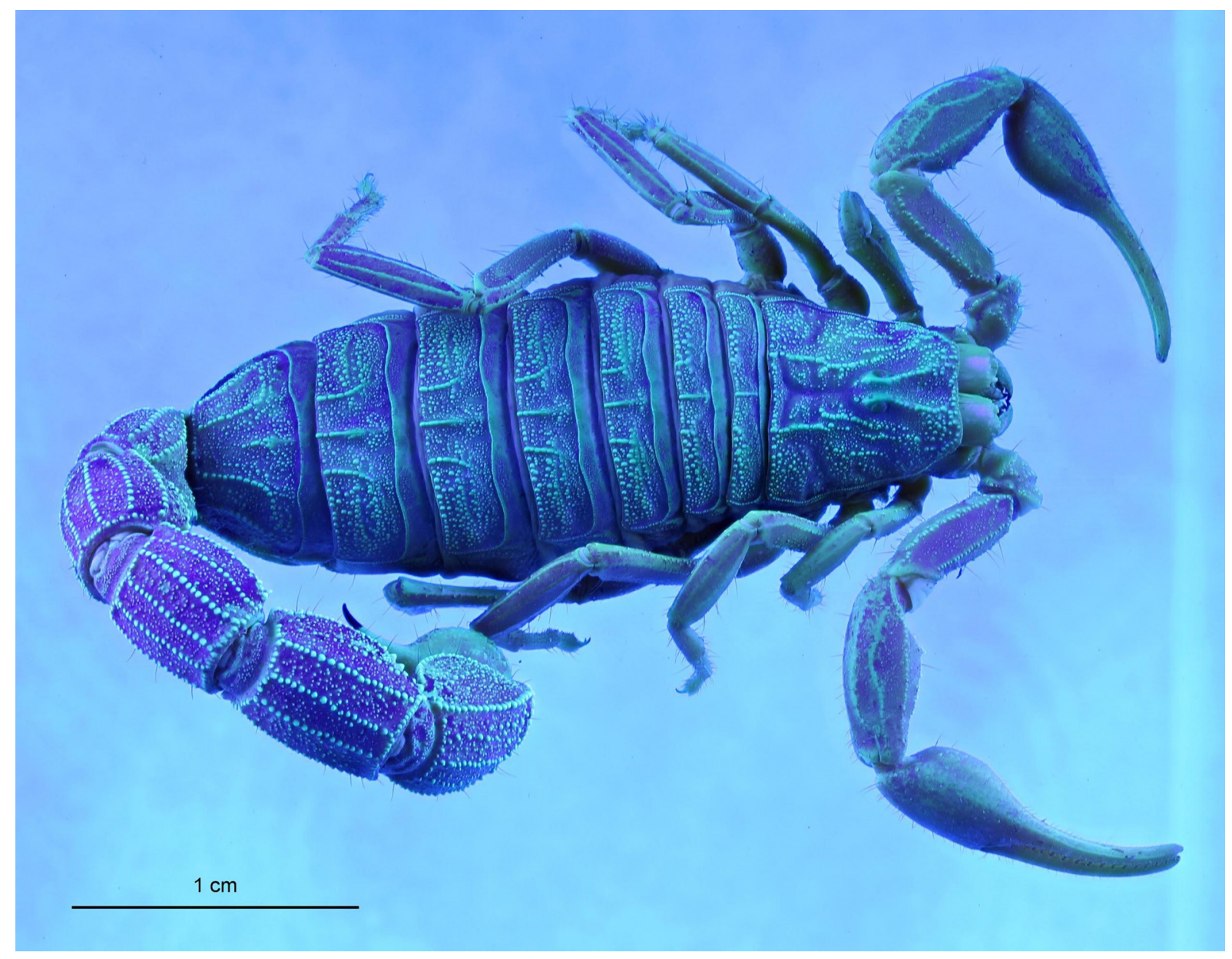

Fig. 4.1. Scorpion pictured in UV fluorescence. Focus stacking image. 


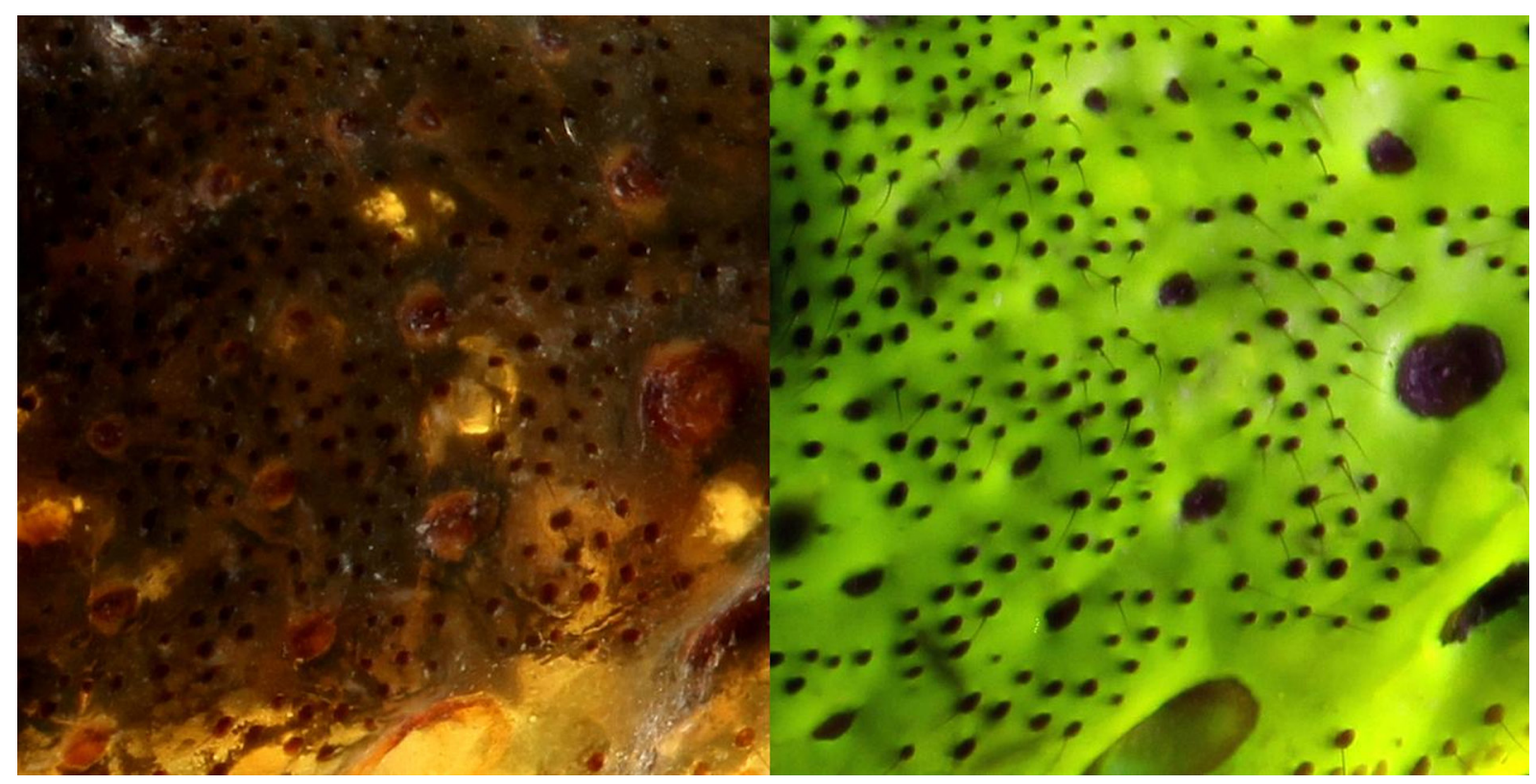

Fig. 4.2. Detail of a spider in white light on the left and in fluorescence on the right. Focus stacking image.

Another application is understanding animal vision. Humans have a vision limited to a spectrum from approximately $400 \mathrm{~nm}$ to $700 \mathrm{~nm}$, while many insects, reptiles (Fleishman et al. 1993), birds (Burkhardt 1989, Honkavaara 2002) and some mammals (Hogg et al. 2011; Jacobs 1992; Douglas \& Jeffery 2014) can see in UV or infrared (IR), and/or are blind to part of the visible spectrum. Digitising specimens using multispectral enables us to see them as they see each other.

Furthermore, UV can be a tool to differentiate cryptic species (Ze-Lin et al. 2012) and in general multispectral allows to differentiate materials. For example, it is used in painting analysis to differentiate 'titanium white' from 'lead white' (Cosentino 2015; Pronti et al. 2017). A general reflectance spectrum can be extracted from multispectral images and can also help to identify or to diagnose the composition of different materials. In remote sensing it enables to distinguish between clear water and turbid water (Nguyen 2012). Classification algorithms can also be used, which in the past have helped to classify patterns of peacock feathers (Medina et al. 2015).

Near infrared (NIR) enables us to see through a thin layer of surface of the specimen, allowing the removal of colour information in some cases (Fig. 4.3) or to see inside a puparium to observe the development of the larva inside (Moran \& Parker 2016).

\subsection{Material and methods}

Multispectral imaging at RBINS and RMCA is used in combination with photography, focus stacking and SfM. It can also be used successfully in combination with RTI (Kotoula 2012, 2015; Kotoula \& Earl 2014) and PLD (Van der Perre \& Hameeuw 2015), but this is still under development at RBINS and RMCA.

For multispectral digitisation, a modified DSLR is used, paired with a multispectral dedicated lens. A modified DSLR is a camera of which the IR cut filter has been removed in order to let pass the full spectrum, so the CMOS is able to capture it completely. CMOS are silicon sensors, therefore the spectrum is limited to approximately 200-1100 nm (Gilblom \& Yoo 2004; Richards 2005), although some modern CMOS are in hybrid materials which can allow extended sensitivity (Ohta 2017; Richards 2005). As 


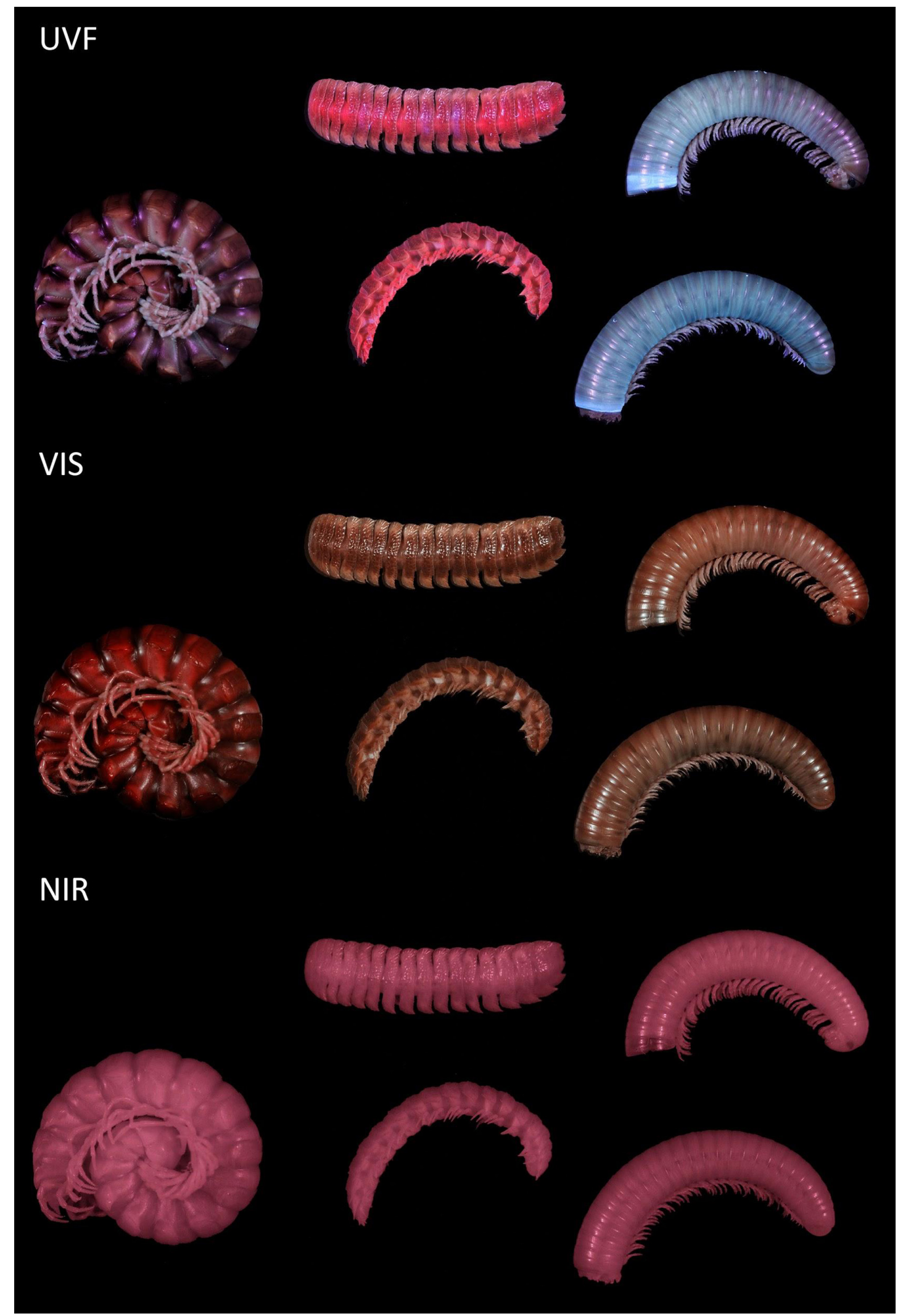

Fig. 4.3. Diplopods in UV fluorescence on top, in white light in the middle, in NIR at the bottom. UV fluorescence show that diplopods can fluoresce in different ways (blue, orange or not at all). NIR show the diplopods without the external pigmented layer. 


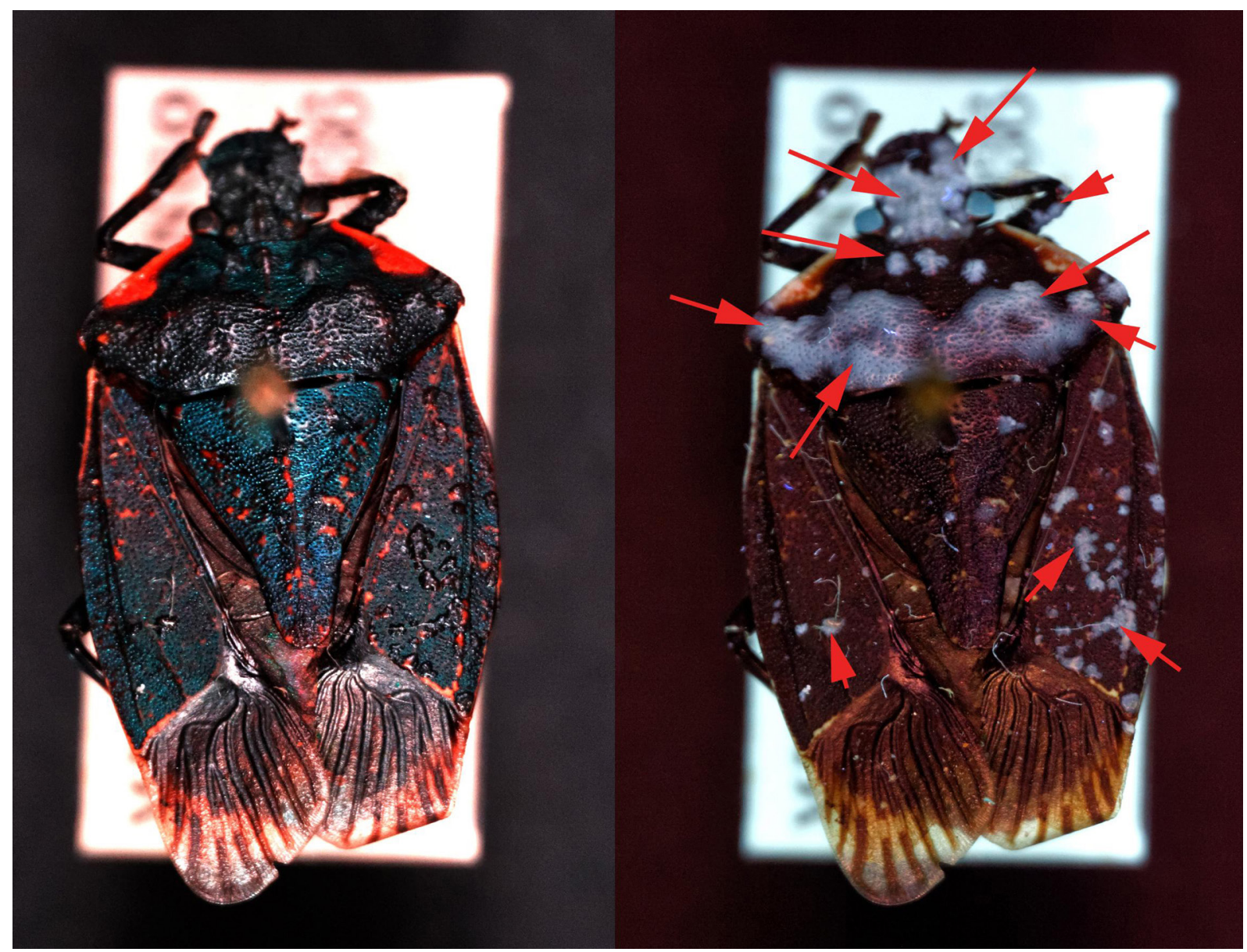

Fig. 4.4. Specimen of the Pentatomidae, Halyomorpha sp. On the left in white light, on the right under UV fluorescence. The red arrows show the parts affected by fungus on the specimen, that are only visible in UV fluorescence.

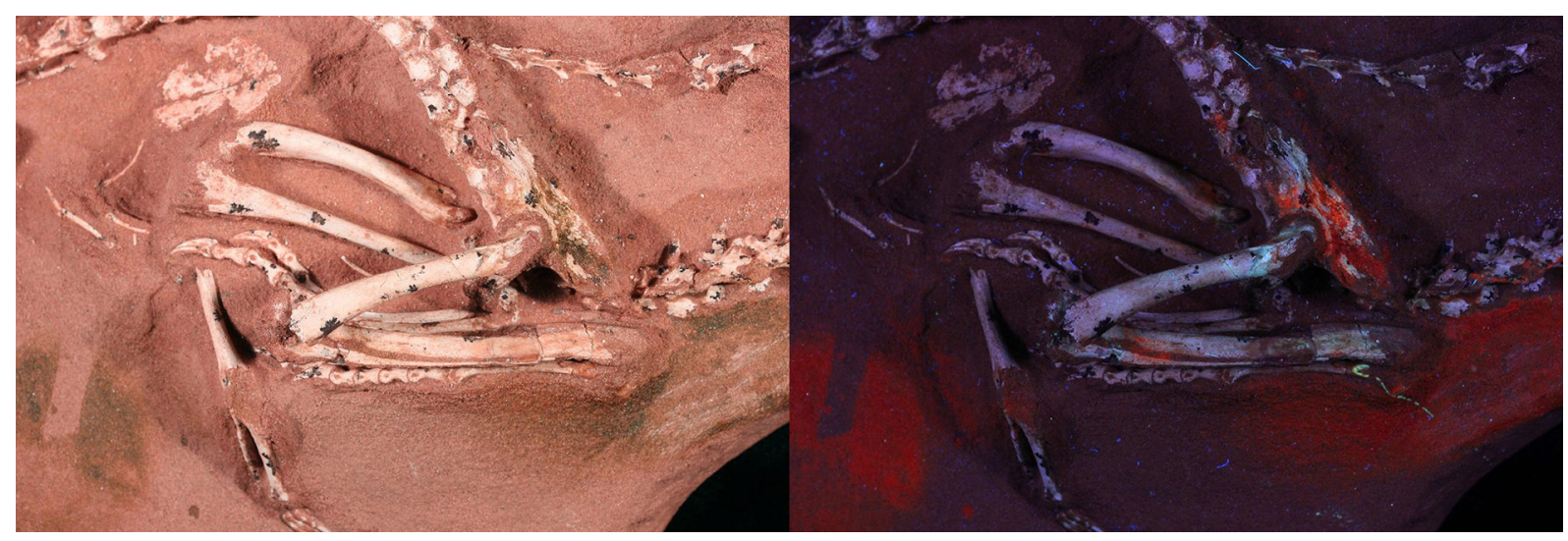

Fig. 4.5. Detail of the Halszkaraptor fossil from Mongolia. In white light on the left, in UV fluorescence on the right. The UV fluorescence image displays restorations of the fossils and treatment applied to preserve it. 
DSLRs are not actually meant to be used for MSI imaging, the actual sensitivity varies depending on the camera model.

For focus stacking photogrammetry in UV, $365 \mathrm{~nm}$ LEDs issued from the manicure industry were used (Brecko et al. 2016). For multispectral photography and SfM (of medium and large specimens), two LED panels with 16 wavelengths from near UV to near infrared (NIR) were used.

Future works include the development of a low-cost setup adapted to museum needs for both SfM and RTI, as dedicated multispectral setups are expensive and not always adapted.

\subsection{Collections}

Multispectral is relatively new to the digitisation world in natural history collections. It has an infinite potential of applications. With the project 3Dspectral, RBINS and RMCA have begun exploring the potential applications to the different collections hosted.

Uses include conservation (Fig. 4.6), enhancing legibility of specimens (Fig. 4.7) and materials analysis (Fig. 4.8).

Using multispectral SfM allows to map the information revealed in multispectral in a three-dimensional way (Fig. 4.9), which can be a lot of help for condition reports. Multispectral SfM can also be used to improve the quality of 3D models (Mathys et al. 2019).

\subsection{References}

Alieva N.O., Konzen K.A., Field S.F., Meleshkevitch E.A., Hunt M.E., Beltran-Ramirez V., Miller D.J., Wiedenmann J., Salih A. \& Matz M.V. 2008. Diversity and evolution of coral fluorescent proteins. PLoS ONE 3 (7): e2680. https://doi.org/10.1371/journal.pone.0002680

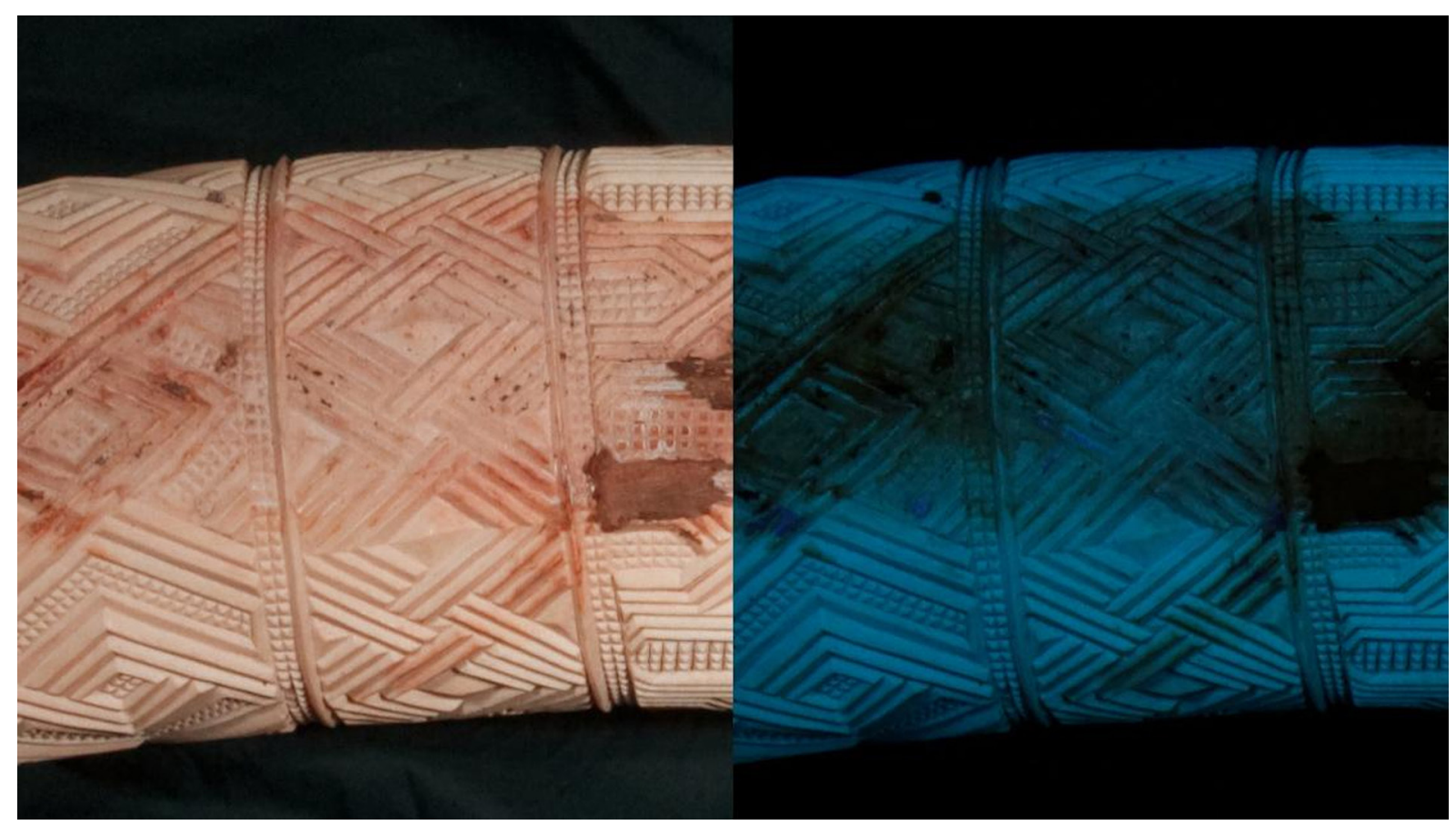

Fig. 4.6. Decorated ivory tusk suffering from humidity. Although visible under white light, the damaged parts are clearly enhanced by UV fluorescence (on the right). 


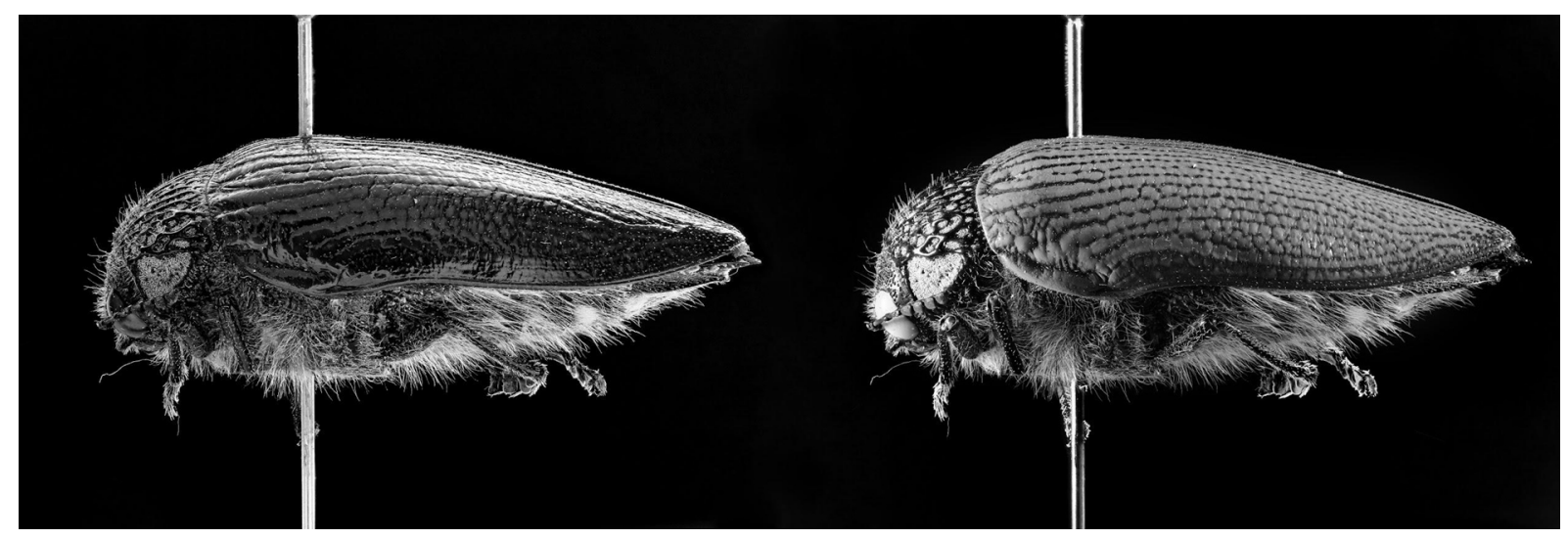

Fig. 4.7. Enhancing the legibility of a specimen. The picture on the left represents the specimen captured under white light, while the picture on the right displays the specimen under UV light. Part of the reflections is reduced under UV light allowing to display more contrasted structures.

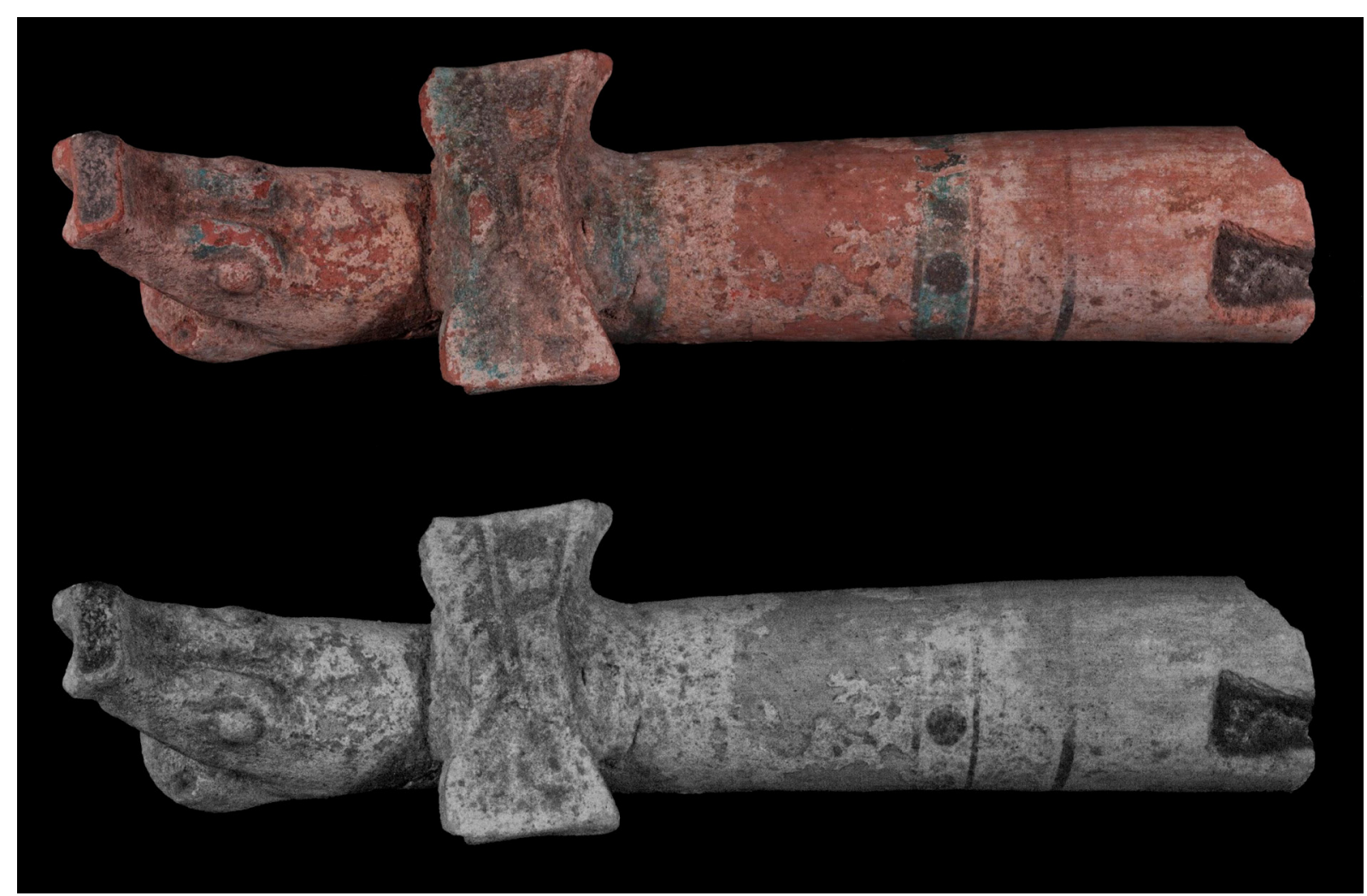

Fig. 4.8. Pre-Columbian handle of an incense shovel from the Royal Museum of Art and History (RMAH) collections, on top in white light, at bottom in infrared. The infrared image only displays carbon-based pigment in black, the other pigments used are transparent to infrared. 
Brecko J., Mathys A., Dekoninck W., De Ceukelaire M., Van den Spiegel D. \& Semal P. 2016. Revealing invisible beauty, ultra detailed: the influence of low-cost UV exposure on natural history specimens in 2D+ digitisation. PLoS ONE 11 (8): e0161572. https://doi.org/10.1371/journal.pone.0161572

Brunton C. 1998. The evolution of ultraviolet patterns in European Colias butterflies (Lepidoptera, Pieridae): a phylogeny using mitochondrial DNA. Heredity 80: 611-616.

https://doi.org/10.1046/j.1365-2540.1998.00336.x

Burkhardt D. 1989. UV vision: a bird's eye view of feathers. Journal of Comparative Physiology 164, 787-796. https://doi.org/10.1007/BF00616750

Cosentino A. 2015. Effects of different binders on technical photography and infrared reflectography of 54 historical pigments. International Journal of Conservation Science 6 (3): 287-298.

Douglas R. H. and Jeffery G. 2014. The spectral transmission of ocular media suggests ultraviolet sensitivity is widespread among mammals. Proceedings of the Royal Society B 281: 20132995.

https://doi.org/10.1098/rspb.2013.2995

Fleishman, L., Loew, E. \& Leal, M. 1993. Ultraviolet vision in lizards. Nature 365, 397.

https://doi.org/10.1038/365397a0

Gilblom D.L. \& Yoo S.K. 2004. Infrared and ultraviolet imaging with a CMOS having layered photodiodes. In: Sampat N., Blouke M.M. \& Motta R.J. (eds) Proceedings of SPIE - The International Society for Optical Engineering 5301, Sensors and Camera Systems for Scientific, Industrial, and Digital Photography Applications V. https://doi.org/10.1117/12.528427

Goutte S., Mason M.J., Antoniazzi M.M., Jared C., Merle D., Cazes L., Toledo L.F., el-Hafci H., Pallu S., Portier H., Schramm S., Gueriau P. \& Thoury M. 2019. Intense bone fluorescence reveals hidden patterns in pumpkin toadlets. Scientific Reports 9, 5388. https://doi.org/10.1038/s41598-019-41959-8

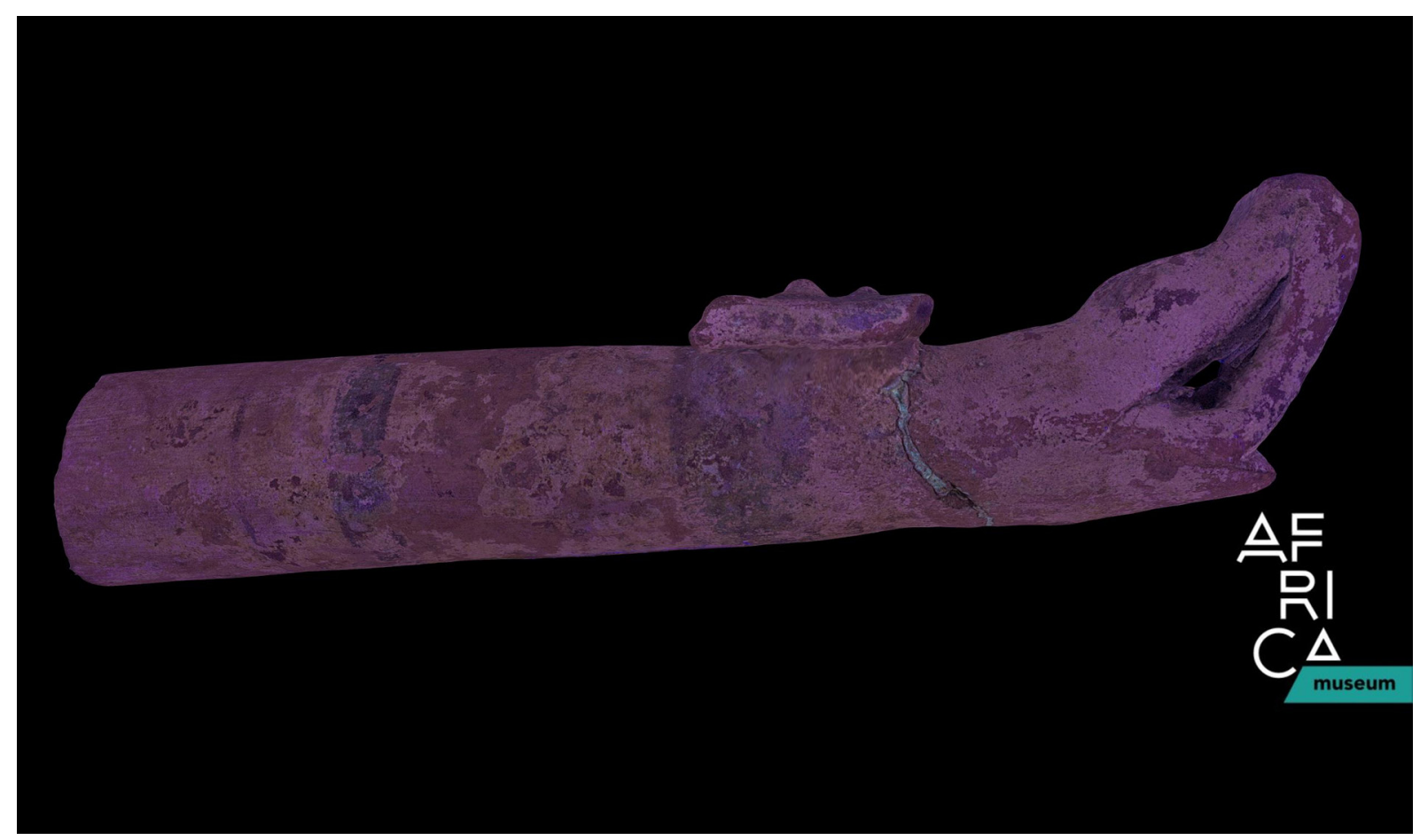

Fig. 4.9. Pre-Columbian handle of an incense shovel from the Royal Museum of Art and History collections. UV fluorescence photogrammetry model. In this case, fluorescence enables to enhance the glue (fluorescing in green). https://sketchfab.com/models/2d82a98be64c48b89cada459b81bd0ab 
Gruber D.F., Sparks J.S. 2015. First Observation of Fluorescence in Marine Turtles. American Museum Novitates 2015 (3845): 1-8.

Hennen D. 2012. A calm millipede's UV fluorescence. Normal Biology blogpost available from http://normalbiology.blogspot.be/2012_03_01_archive.html [accessed 28 Aug. 2018].

Hogg C., Neveu M., Stokkan K-A., Folkow L., Cottrill P., Douglas R., Hunt D.M. \& Jeffery G. 2011. Arctic reindeer extend their visual range into the ultraviolet. Journal of Experimental Biology 214 (12), 2014-2019. https://doi.og/10.1242/jeb.053553

Honkavaara J., Koivula M., Korpimäki E., Siitari H. \& Viitala J. 2018. Ultraviolet vision and foraging in terrestrial vertebrates. Oikos 98 (3): 505-511.

Jacobs G. 1992. Ultraviolet Vision in Vertebrates. American Zoologist 32 (4), 544-554.

Kohler A.M., Olson E.R., Martin J.G. \& Spaeth Anich P. 2019. Ultraviolet fluorescence discovered in New World flying squirrels (Glaucomys). Journal of Mammalogy 100 (1): 21-30.

https://doi.org/10.1093/jmammal/gyy177

Kotoula E. \& Earl G. 2014. Integrated RTI approaches for the study of painted surfaces. Computer Applications and Quantitative methods in Archaeology 45911.

Available from http://caa2014.sciencesconf.org/45911/ [accessed 10 Jan. 2020].

Kotoula E. 2015. Ultraviolet RTI. Available from http://acrg.soton.ac.uk/blog/4175/ [accessed 31 Aug. 2018].

Kotoula E. 2012. Infrared RTI: experimentation towards the development of multispectral RTI. Available from http://acrg.soton.ac.uk/blog/1569/ [accessed 28 Aug. 2018].

Martin M., Meylan S., Gomez D. \& Le Galliard J.F. 2013. Ultraviolet and carotenoid-based colouration in the common lizard Zootoca vivipara (Squamata: Lacertidae) in relation to age, sex, and morphology. Biological Journal of the Linnean Society 110:128-141. https://doi.org/10.1111/bij.12104

Mathys A., Semal P., Brecko J. \& Van den Spiegel D. 2019. Improving 3D photogrammetry models through spectral imaging: Tooth enamel as a case study. PLoS ONE 14 (8): e0220949. https://doi.org/10.1371/journal.pone.0220949

Medina J.M., Díaz J.A \& Vukusic P. 2015. Classification of peacock feather reflectance using principal component analysis similarity factors from multispectral imaging data. Optics Express 23, 1019810212. https://doi.org/10.1364/OE.23.010198

Moran Z.R. \& Parker A.G. 2016. Near infrared imaging as a method of studying tsetse fly (Diptera: Glossinidae) pupal development. Journal of Insect Science 16 (1): 72, 1-9.

https://doi.org/10.1093/jisesa/iew047

Nguyen D.D. 2012. Water body extraction from multispectral image by spectral pattern analysis. International Archives of the Photogrammetry, Remote Sensing and Spatial Information Sciences XXXIX-B8: 181-186. https://doi.org/10.5194/isprsarchives-XXXIX-B8-181-2012

Nowogrodzki A. 2017. First fluorescent frog found. Nature 543 (7645).

Ohta J. 2017. Smart CMOS Image Sensors and Applications. CRC Press, Boca Raton.

Pearson G. 2013. Luminous beauty: the secret world of fluorescent arthropods. Wired. Available from http://www.wired.com/2013/11/arthropods-are-having-a-secret-rave/ [accessed 28 Aug. 2018].

Pecháček P., Stella D. \& Kleisner K. 2019. A morphometric analysis of environmental dependences between ultraviolet patches and wing venation patterns in Gonepteryx butterflies (Lepidoptera, Pieridae). Evol Ecol 33, 89-110. https://doi.org/10.1007/s10682-019-09969-0 
Pronti L., Felici A.C., Ménager M., Vieillescazes C. \& Piacentini, M. 2017. Spectral behavior of white pigment mixtures using reflectance, ultraviolet-fluorescence spectroscopy, and multispectral imaging. Applied Spectroscopy 71 (12): 2616-2625. https://doi.org/10.1177/0003702817717969

Prötzel D., Heß M., Scherz M. D., Schwager M., va not Padje A. \& Glaw F. 2018. Widespread bonebased fluorescence in chameleons. Scientific Reports 8: 698.

Richards A. 2005. Infrared spectral selection; it begins with the Detector. Photonics Handbook 2005. Available from https://www.photonics.com/a25132/Infrared_Spectral_Selection_It_Begins_with_the [accessed 28 Aug. 2018].

Salih A., Larkum A., Cox G., Kuhl M. \& Hoegh-Guldberg O. 2000. Fluorescent pigments in corals are photoprotective. Nature 408: 850-853. https://doi.org/10.1038/35048564

Stella D., Pecháček P., Meyer-Rochow V.B. \& Kleisner K. 2018. UV reflectance is associated with environmental conditions in Palaearctic Pieris napi (Lepidoptera: Pieridae). Insect Science 25: 508-518. https://doi.org/10.1111/1744-7917.12429

Siebeck U.E., Parker A.N., Sprenger D., Mäthger L.M. \& Wallis G. 2010. A species of reef fish that uses ultraviolet patterns for covert face recognition. Current Biology 20 (5): 407-410. https://doi.org/10.1016/j.cub.2009.12.047

Taboada C., Brunetti A.E., Pedron F.N., Neto F.C., Estrin D.A., Bari S.E., Chemes L.B., Peporine Lopes N., Lagorio M.G., \& Faivovich J. 2017. Proceedings of the National Academy of Sciences. 114 (14) 3672-3677. https://doi.org/10.1073/pnas.1701053114

Thompson M.E., Saporito R.A., Ruiz-Valderrama D.H., G.F. Medina-Rangel \& Donnelly M.A. 2019. A field-based survey of fluorescence in tropical tree frogs using an LED UV-B flashlight. Herpetology Notes 12: 987-990.

Van der Perre A. \& Hameeuw H. 2015. La création d'images multi-spectrales: les portraits romains du Fayoum. In: Delvaux L., Therasse I. (eds) Sarcophages: Sous les étoiles de Nout: 164-165. Éditions Racine, Brussels.

Ze-Lin W., Ngan-Kee N., Teo S.L.M. \& Parra-Velandia F.J. 2012. Fluorescent patterns in some portunus species (Crustacea: Brachyura: Portunidae). Contributions to Marine Science 2012: 135-143. Available from https://www.yumpu.com/en/document/read/38378257/wong-ze-lin-serena-teo-lay-ming-tropicalmarine-science-institute [accesed 30 Mar. 2020] 


\section{Post-processing}

All the previously mentioned techniques in the 3D digitisation chapter allow users to create 3D models that are ready to use. However, this output can be considered the same as a picture right after it is taken using the automatic settings. It can be satisfactory when used in this way, but if some post-processing is done the result can be a lot better. Moreover, 3D models are often too large to share without being reduced in size, just like in the old days when it was absolutely necessary to reduce the file size of pictures when sharing online, using in presentations, etc.

In this short chapter the different techniques used to post-process pictures and specifically 3D models are discussed. This is of course not the holy grail of post-processing workflow, nor the only possible way to do it, but it describes the current worflow of the digitisation cells of RBINS and RMCA.

It is very important that these steps are carefully written down in a metadata file that accompanies the 3D model. Because if the model is used later on in research studies like geometric morphometrics it is important that one knows what happened with model during the post-processing to take this into account in the performed study. Also, it is necessary to write down which software is used and even which version, as algorithms might change over time. Of course, this also implies that a copy of the original dataset without the post-processing is stored.

\subsection{Post-processing of pictures}

Post-processing of pictures can take hours if one wants to have all the details just right and produce perfect show-stopping images time after time. This is not wanted within a digitisation effort to digitise entire collections. But with a very limited amount of time one can improve the output easily and make it accessible for anyone.

Because the images are all taken under the same light conditions, a very limited amount of time is needed to post-process them. The same settings in the post-processing pathway can be used over again or with only slight adjustments if the specimens differ a lot from one another.

The main issue with focus stacking is that sometimes the final picture is a little darker than the original images (Fig. 5.1) so the most common setting that will be adjusted is (micro)contrast and light (Fig. 5.2). If the image is well exposed, only the (micro)contrast will be adjusted if it proves to improve one of the pictures of a series (Figs 5.3-5.4), otherwise the picture is kept as it is. After these adjustments are done, or by directly using the original stacked image, it is processed in batch software. Here the scales matching the magnification are added and a duplicate image is produced at $90 \%$ of the jpeg quality. This
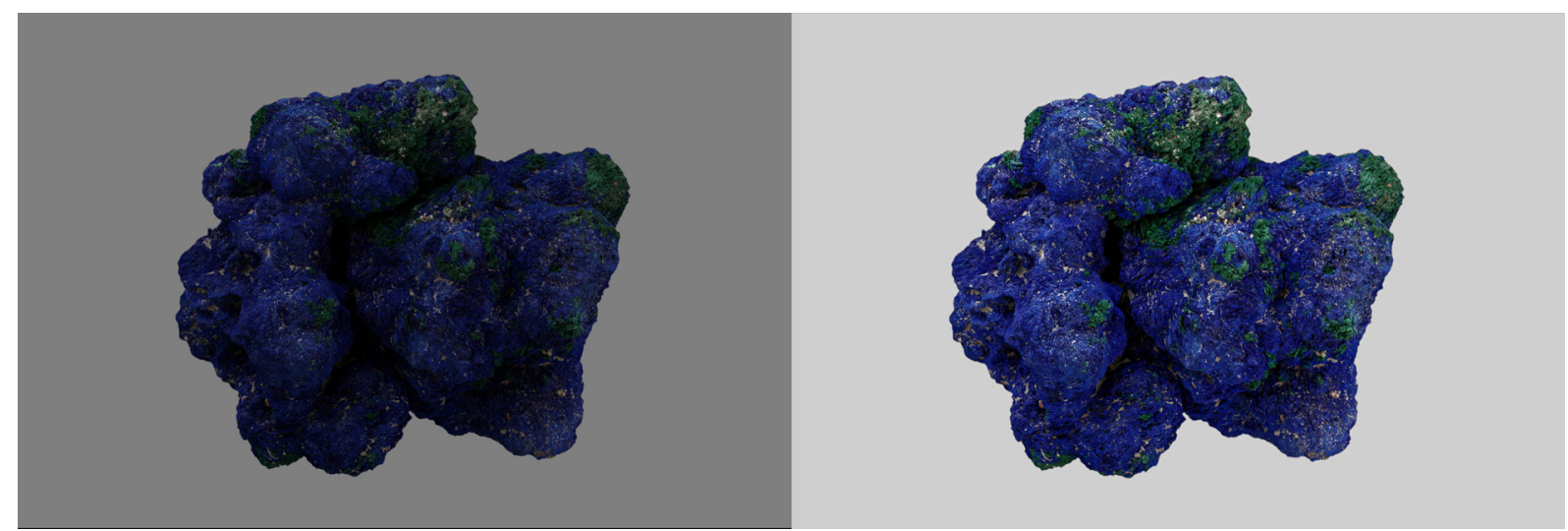

Fig. 5.1. Relighting in DxO OpticsPro 11. The original image is on the left, the post-processed picture on the right. The underexposed image is now corrected without the need to take new images. 


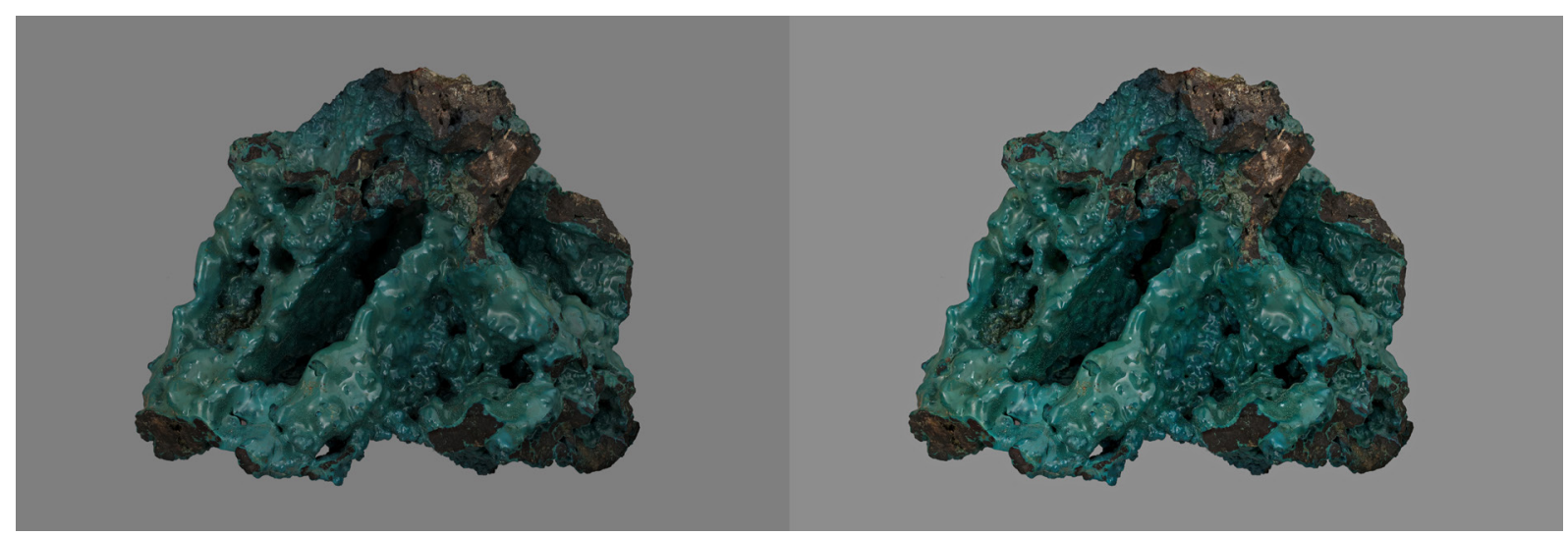

Fig. 5.2. Micro-contrast enhancement and relighting in DxO OpticsPro 11. The original image is on the left, the post-processed picture on the right. The dark area in the middle is now sufficiently exposed, without losing the look and feel of the specimen.

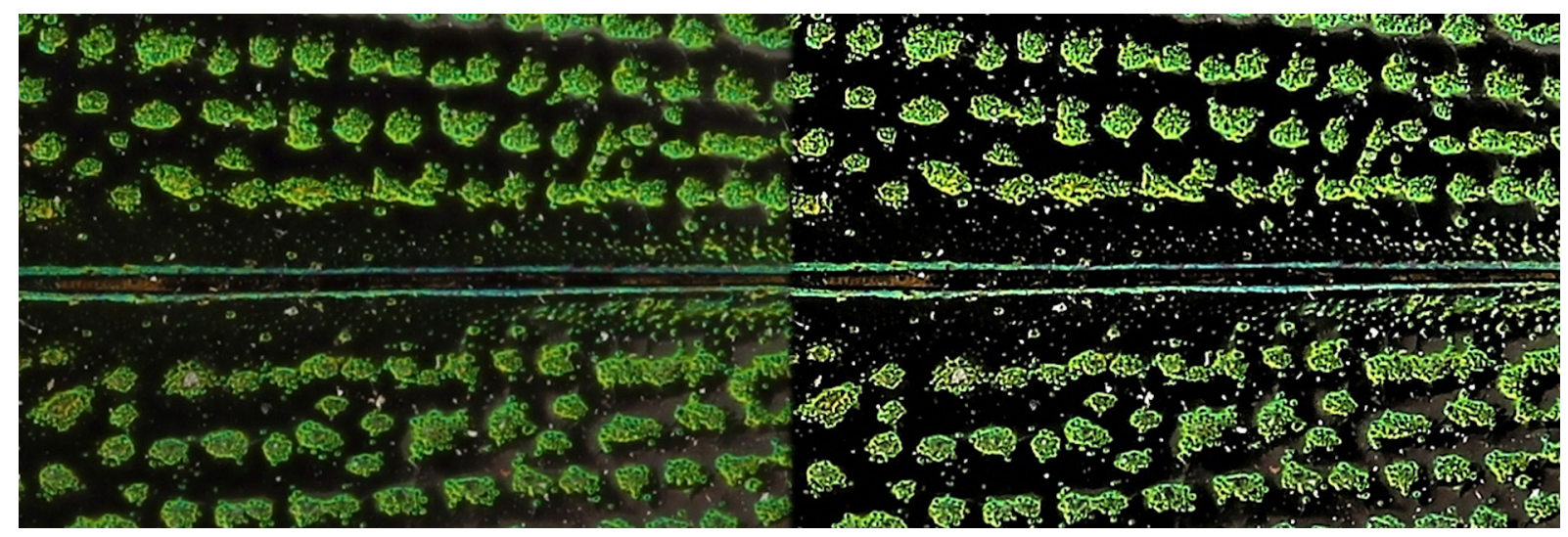

Fig. 5.3. Micro-contrast enhancement in DxO OpticsPro 11. A crop of the original image is on the left, one of the post-processed pictures on the right.

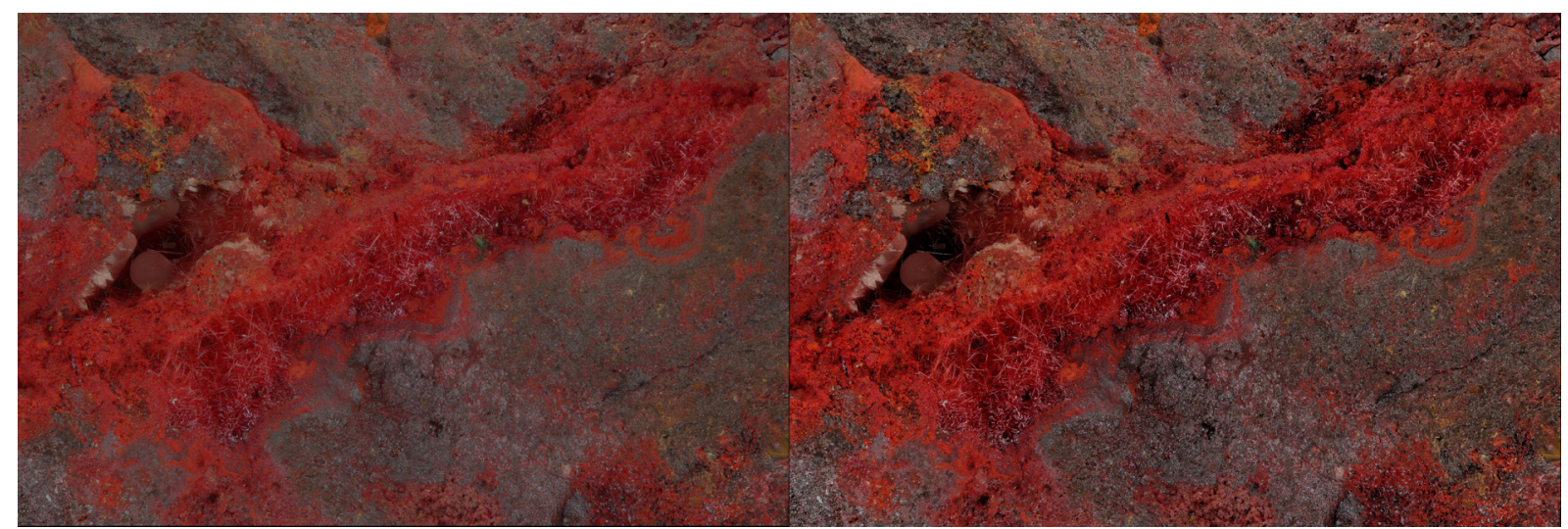

Fig. 5.4. Micro-contrast enhancement in DxO OpticsPro 11. The original image is on the left, the postprocessed picture on the right. The post-processed picture looks more crisp and shows more details than the original one as the washed-out appearance has gone. 
does not visibly change a lot in the details but lowers the file size significantly. It has to be noted that this technique is only for disseminating the data through an online platform, allowing a smaller file size that permits the data to be easily distributed and consulted, even in remote areas without efficient internet connection. As JPEG is a format that is highly compressed, which is not desirable in this case, it is better to use TIF or PNG formats. If the images are used to do 3D reconstruction, such as using SfM, it is better to use TIF formats to preserve the original information of the picture. However, for dissemination it is often necessary to reduce the data, which saves server space when uploading the image to a website and also makes sure that people in remote areas with low bandwidth are able to consult the digitised specimen images. The possible ways of disseminating final pictures can be found in the chapter 'Data distribution'.

The software used to alter the (micro)contrast and light settings is DxO OpticsPro 11, while ImBatch is the software used to add scales (as a watermark) and save as reduced duplicate in a batch process. But obviously other programmes might do the same tricks.

\subsection{Post-processing of 3D models}

Most of the software applications used with one of the discussed 3D techniques allow 3D model exports that are ready to be viewed, measured, shared, etc. But the same rule of thumb can be applied here as with a 2D picture: the 3D model is good but can be improved. It is not always necessary to post-process the obtained 3D model, but it can often help to disseminate a model if it is decimated or certain areas are clearer when noise is eliminated, or a smoothing filter is applied. Several software packages exist to do post-treatment of a 3D model, of which some well-known applications are GOM Inspect (https://www.gom.com/3d-software/gom-inspect.html), MeshLab (http://www.meshlab.net/), MeshMixer (http://www.meshmixer.com/), Geomagic Design X (https://www.3dsystems.com/software/ geomagic-design-x) and Geomagic Wrap (https://www.3dsystems.com/software/geomagic-wrap). It is not the intention of the authors of this paper to go into too much detail on how these different programmes work, but merely to discuss what can or needs to be done after a model is obtained by using a 3D digitisation technique. More thorough workflows can be found in the 'Workflow' section.

Sometimes the produced texture can be too dark, resulting in a dark model. This can easily be solved by correcting the light settings in an image processing software like DxO OpticsPro (Fig 5.5.).

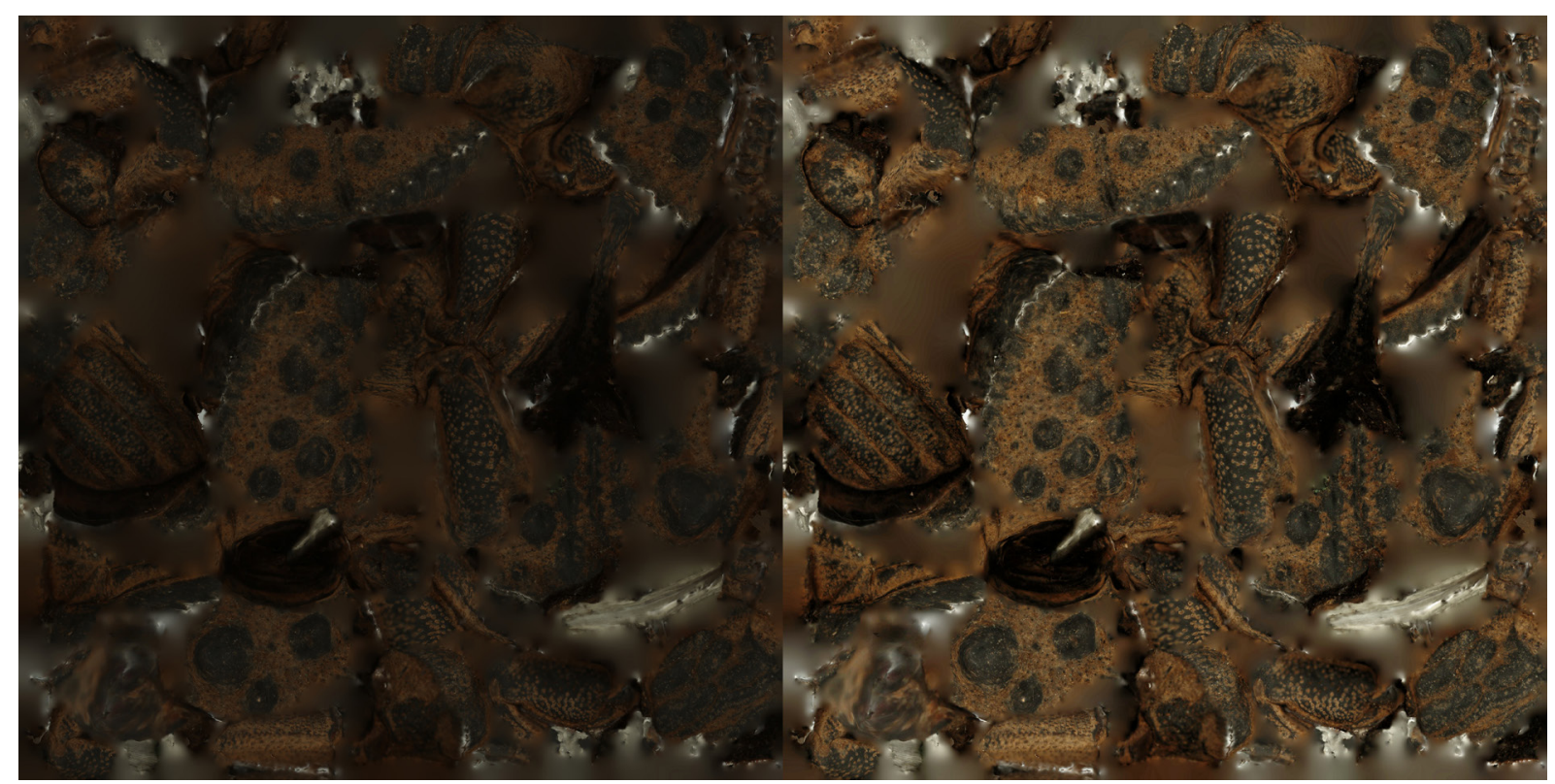

Fig. 5.5. Corrected light settings (right image) for a too-dark texture (left image). 
When highly detailed 3D models are produced, this often results in a lot of faces and vertices. Much more than is necessary for simple web viewing through a portal like SketchFab or when hosted through one's own platform. Therefore, it is wise to decimate the 3D model so it can be viewed smoothly. The trick is to smooth it the maximum without losing valuable information (Fig. 5.6).

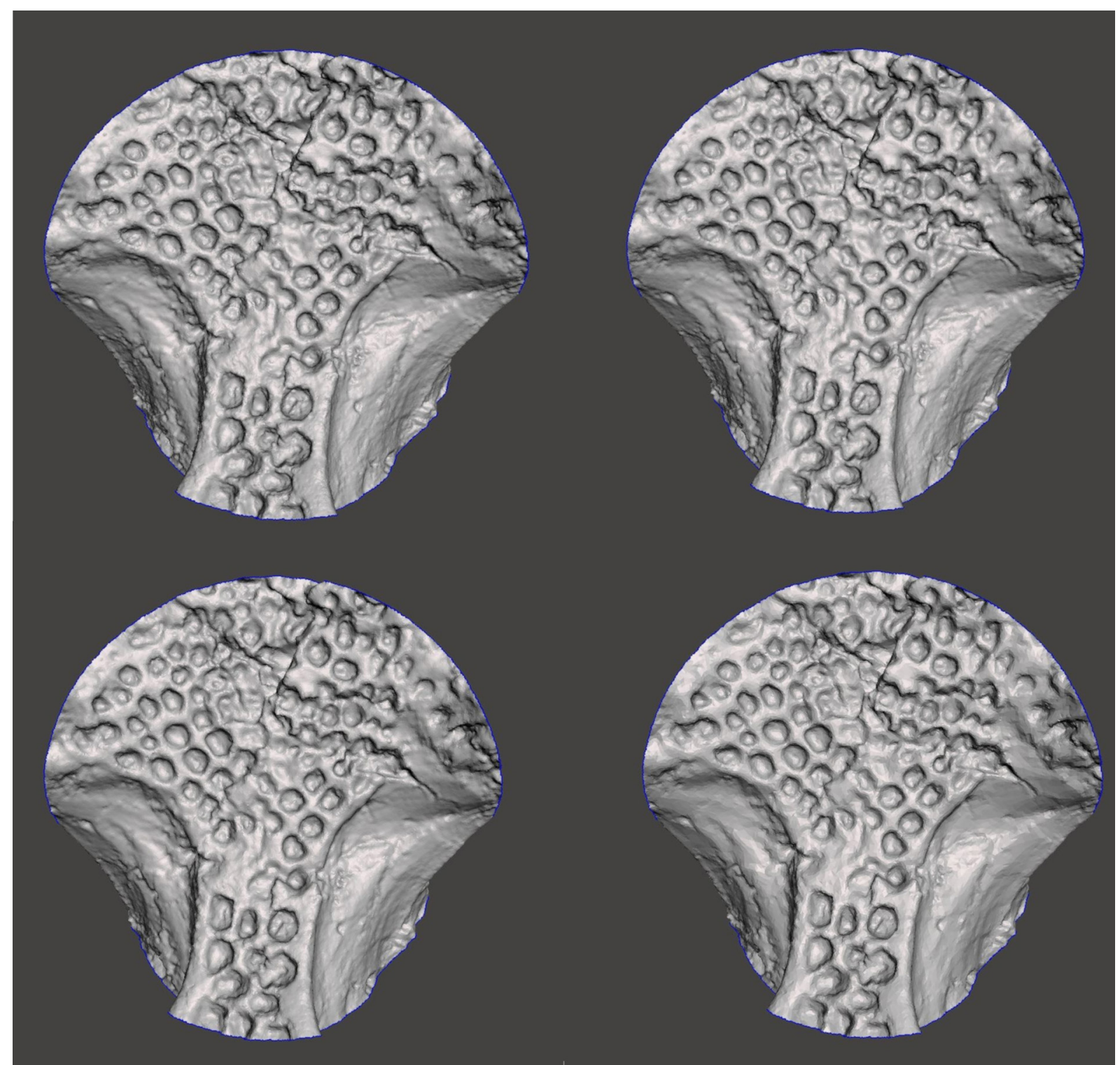

Fig. 5.6. Decimation of a 3D model. The four parts show a 3D model in various degrees of reducing the amount of faces. In the left upper corner is the original and rotating clockwise are the models at $50 \%$, $75 \%$ and $90 \%$ decimation. Until $75 \%$ there is hardly any difference noticeable, while at $90 \%$ the cracks become less deep and the faces become more visible. 


\section{Comparison of the 3D Techniques}

It is clear that for certain collections many different techniques can be used to create a 3D model of a specimen with more-or-less similar results. It is often difficult to judge with the information given per technique which one is better than another in certain circumstances. Therefore, in this chapter the techniques discussed in the previous chapters will be compared to have an overview of the differences between and also within the 3D digitisation techniques.

\subsection{Medium specimens}

The 3D models of a shell recorded by different techniques showed big differences in the results between the high-resolution structured light scanner and the normal one (Fig. 6.1). The SfM-derived model is relatively similar to the high-resolution structured light scanner, but the texture is more accurate (for both sharpness and colour) than the textures from the structured light scanner (Fig. 6.2).

In the case of a digitised skull (Figs 6.3-6.4) the comparison included SfM, CT, six different structured light scanners and two triangulation laser scanners where different parameters were considered. The result showed that CT captures best the deepest structures, while some of the structured light and laser scanners were more precise in terms of the amount of detail. Structured light and laser scanners can achieve similar resolution depending on the scanner specification. Regarding enamel acquisition, CT is the best, while both laser scanners and SfM are the least accurate.
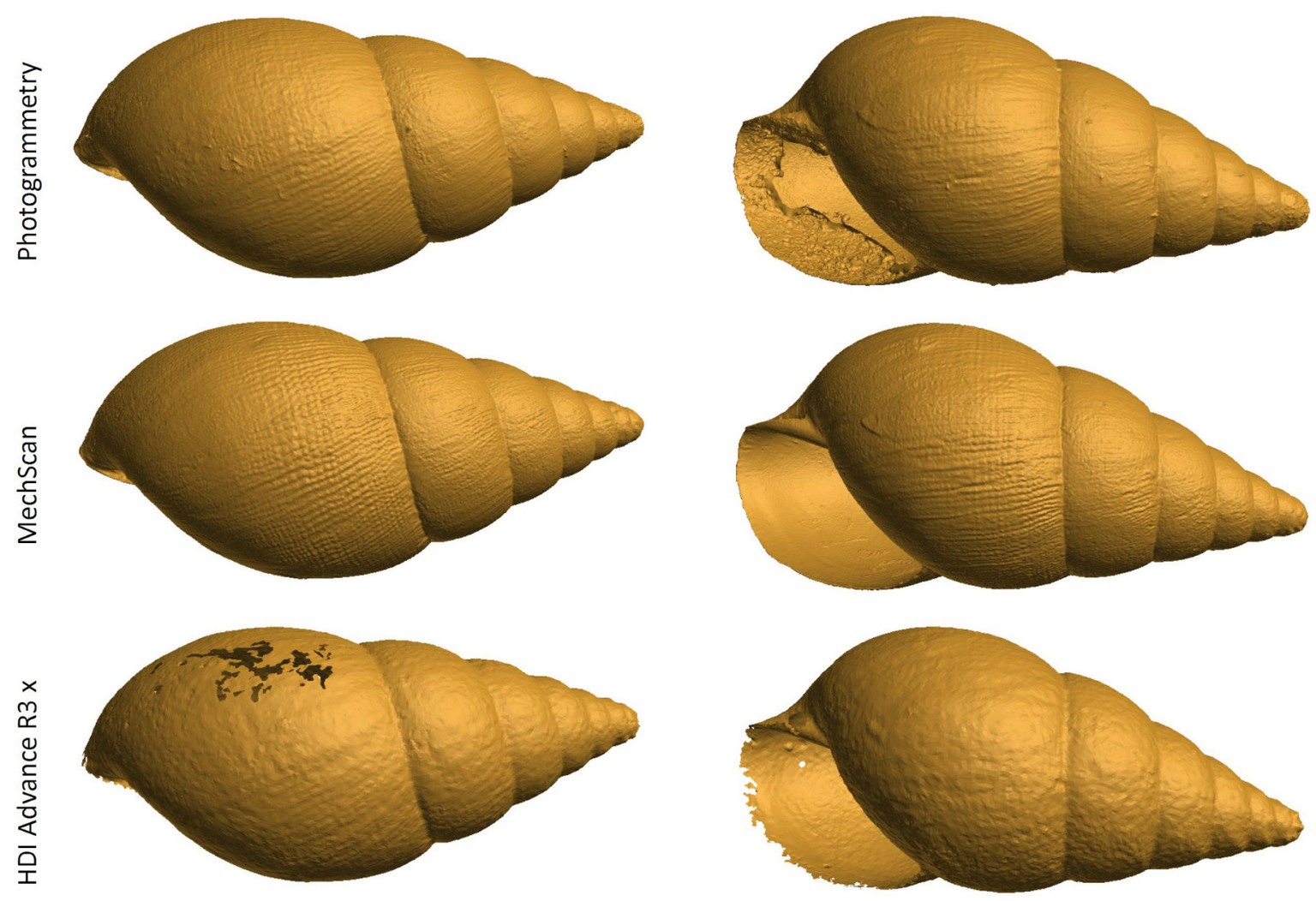

Fig. 6.1. Shell digitised with different methods. The photogrammetry model was captured with a $100 \mathrm{~mm}$ Macro lens and processed with Agisoft Photoscan. The visual comparison of the mollusc shows a similar level of detail between photogrammetry and MechScan for the external surfaces, with still a bit more detail for the MechScan. The HDI Advance has a much lower resolution. 

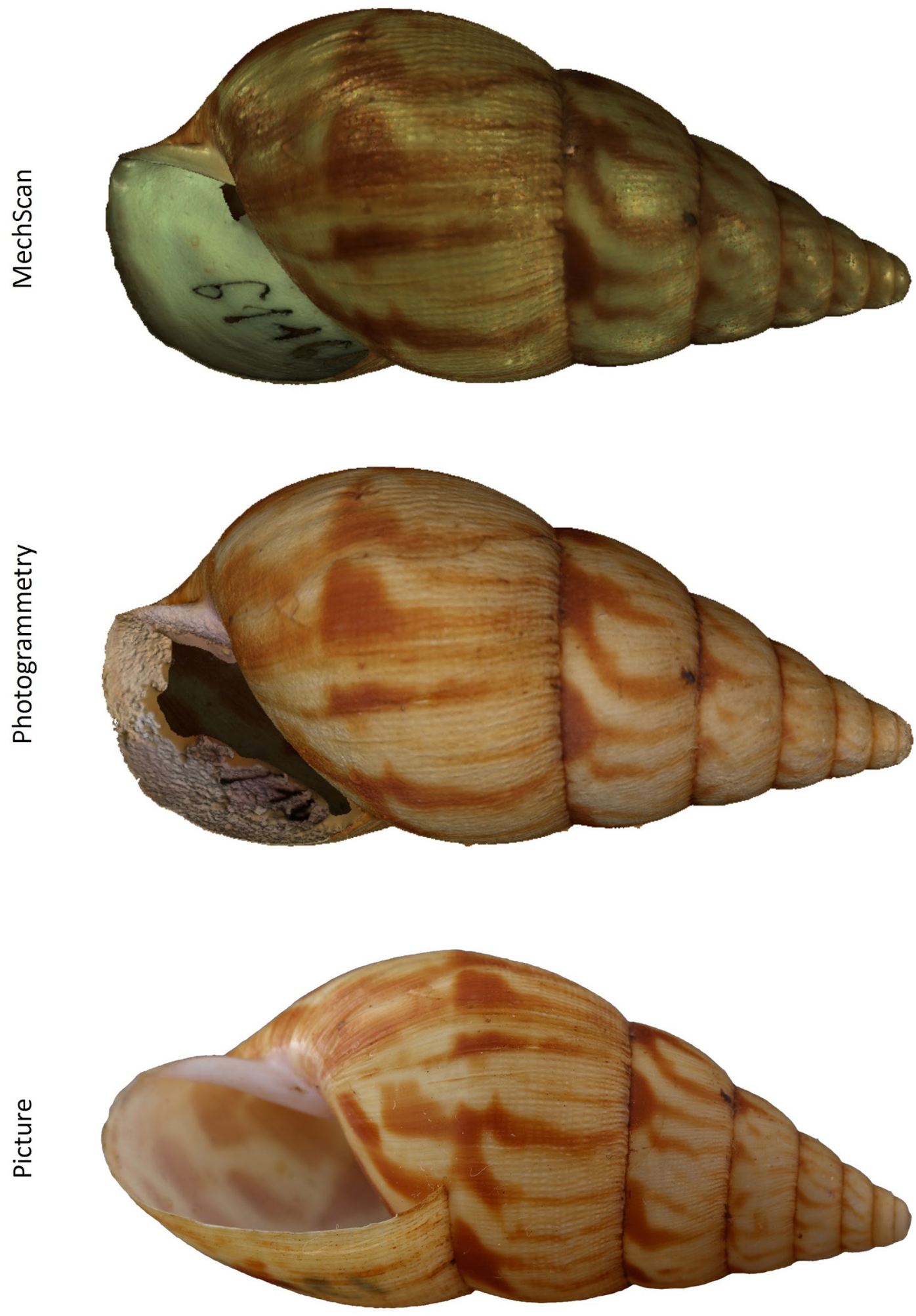

Fig. 6.2. Texture comparison between photogrammetry and MechScan (above) and an actual picture captured by a Canon 700D with $100 \mathrm{~mm}$ macro lens of the shell below. 


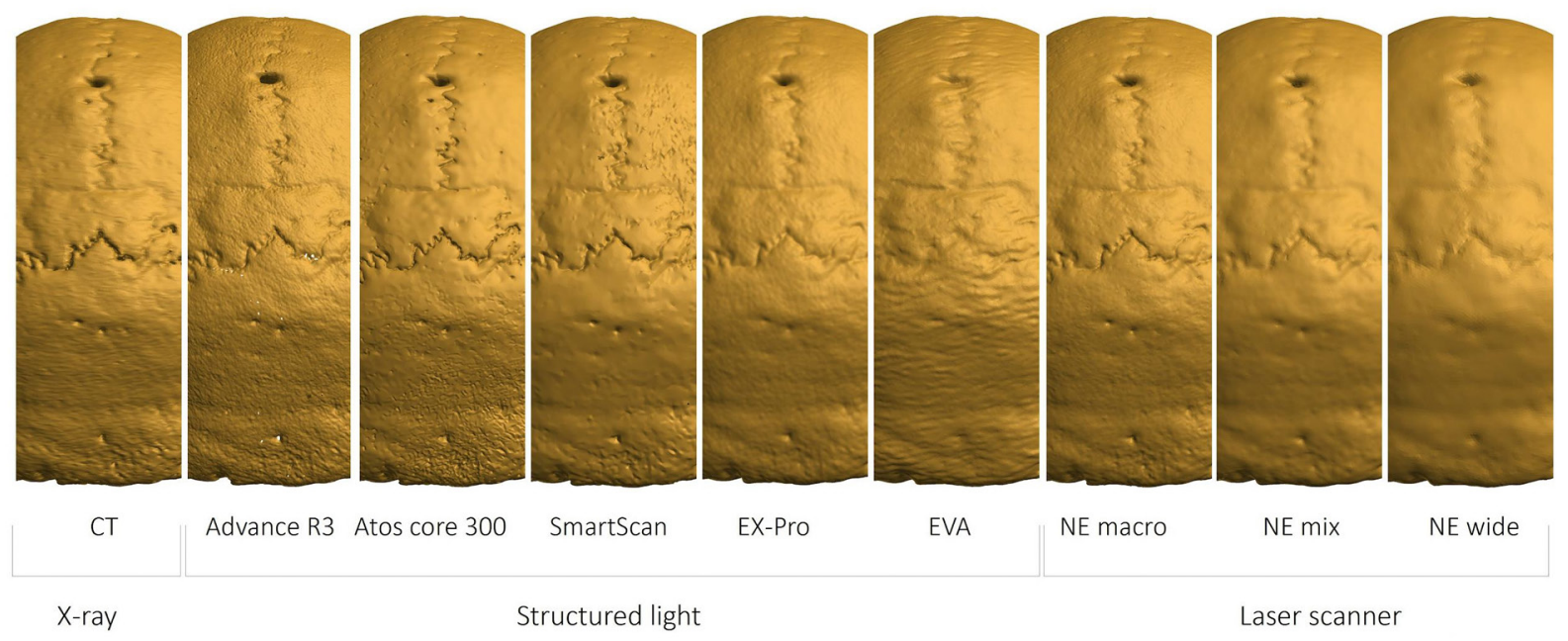

Fig. 6.3. Back view of the skull digitised with different scanners.
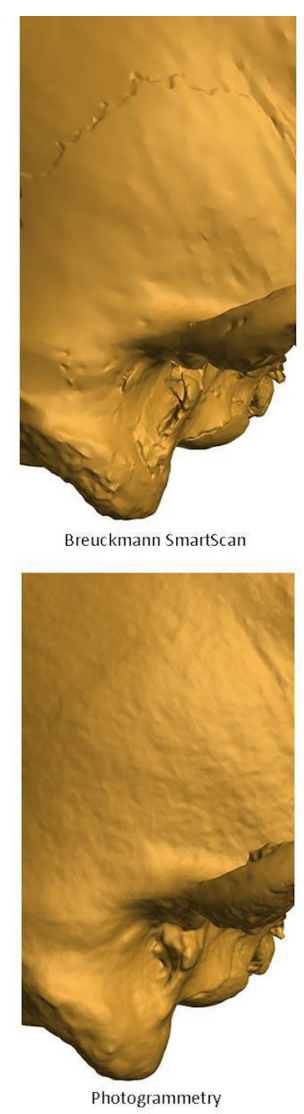

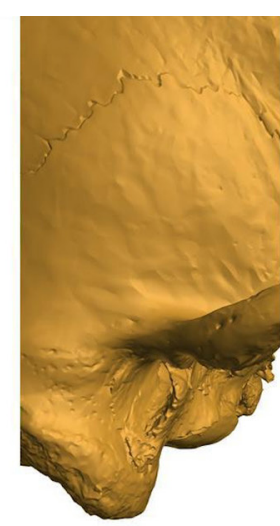

GOM Atos Core 300

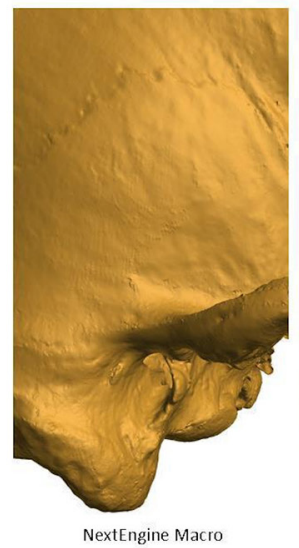

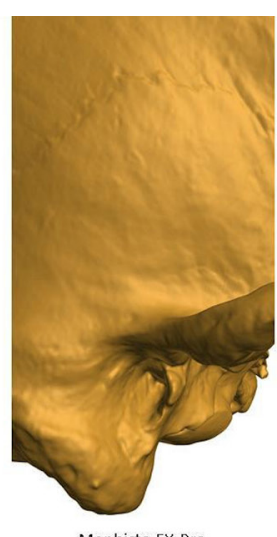

Mephisto EX-Pro

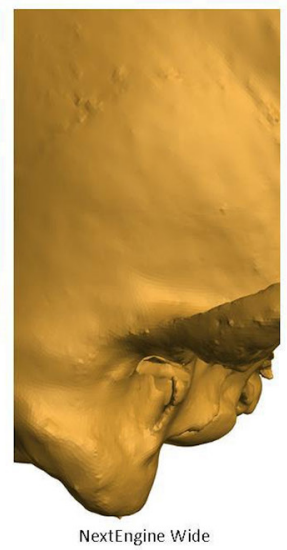

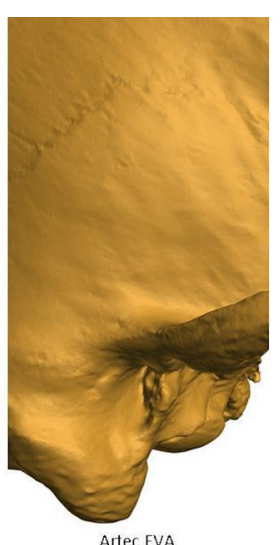

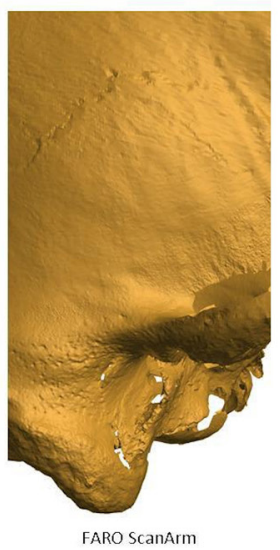

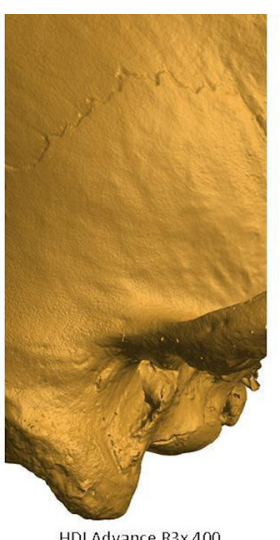

HDI Advance R3 400

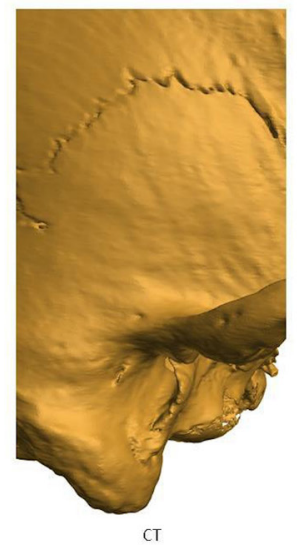

Fig. 6.4. Parietal view of the skull digitised with different scanners. 
The sea urchin was digitised with structured light scanners of different manufacturers and with SfM. Results between the structured light model can be very different from one another (Figs 6.5-6.6), even though it is the same technique. The SfM results were similar to some of the structured light scanners. None of the light scanners were able to capture/mesh correctly the small punctures of the surface of the sea urchin.

Finally, the result of the digitisation of bone retouchers with different camera lenses in SfM showed that results can be very different, depending on the lens used. In the best case the result can be similar to high-resolution structured light scanners or microCT (Fig. 6.7).

All these comparisons showed that a same technique, depending on the instrument and methodology, can generate results with very different resolutions.

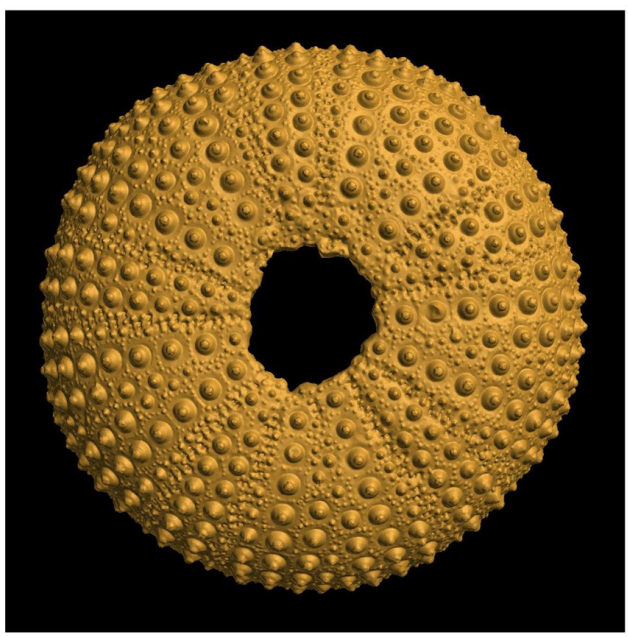

Breuckmann SmartScan Macro

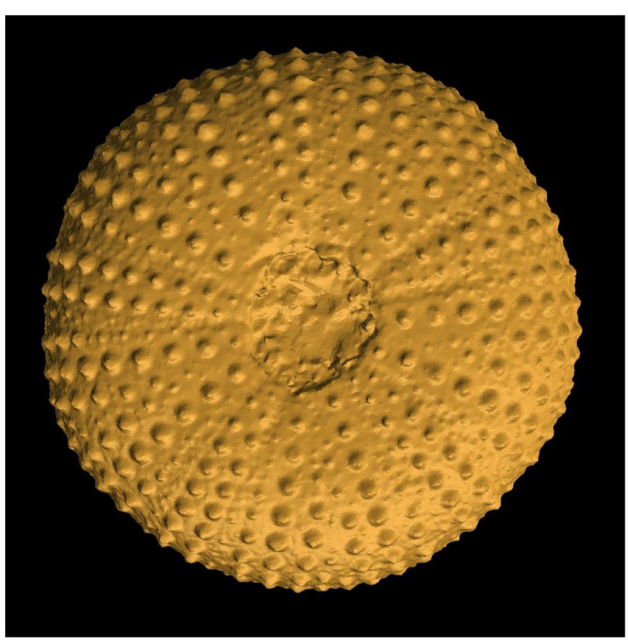

Photogrammetry

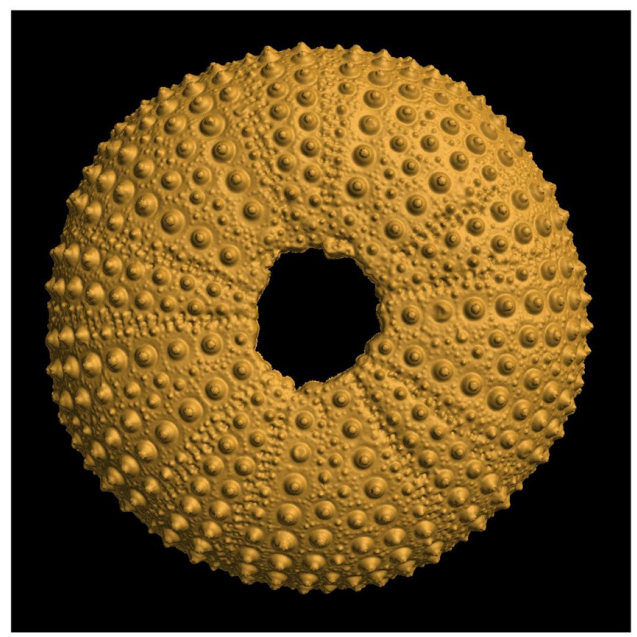

Mechscan

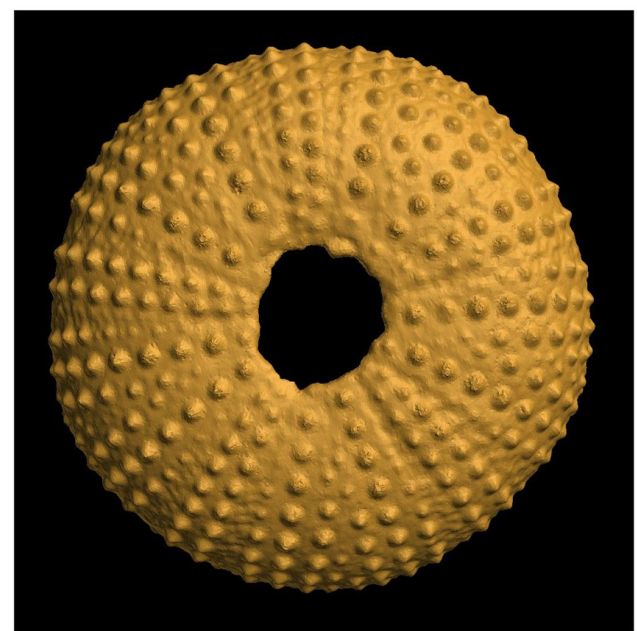

HDI Advance R3

Fig. 6.5. Sea urchin captured by 3 different structured light scanners and by SfM. 


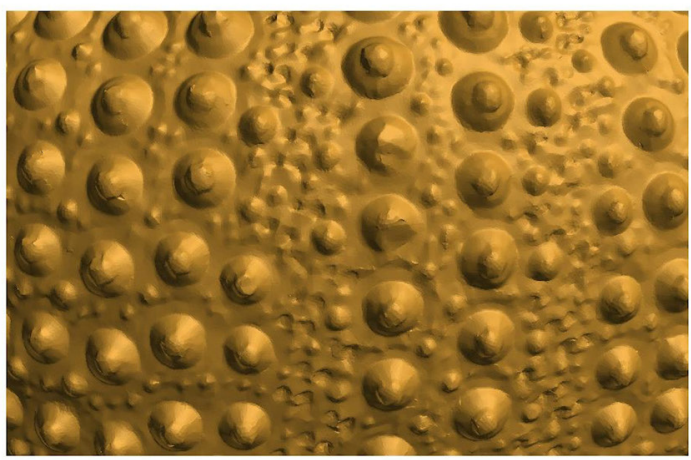

Breuckmann SmartScan Macro

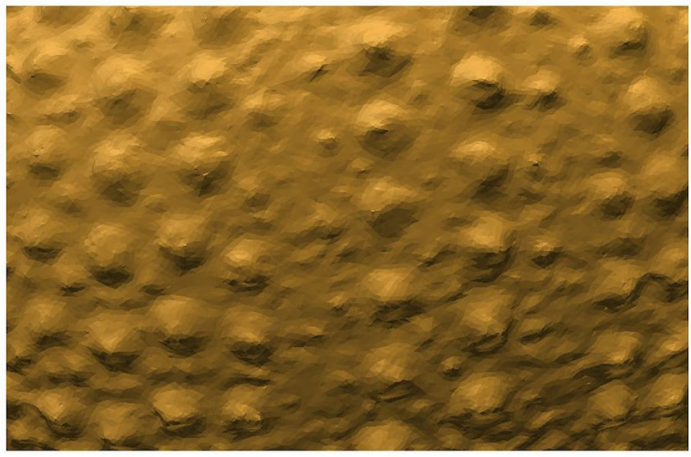

Photogrammetry

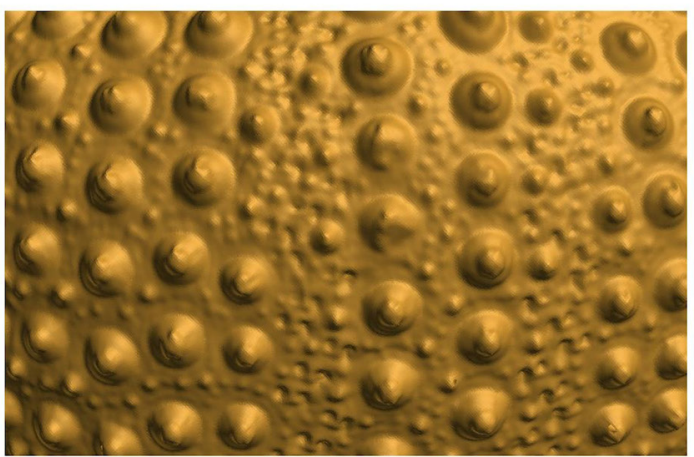

Mechscan

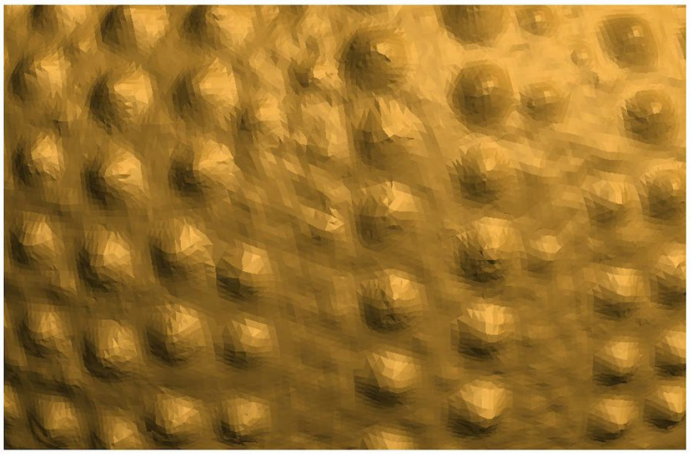

HDI Advance R3

Fig. 6.6. Detailed view of the sea urchins digitised with different equipment. The goal was to digitise the small punctures on the surface as these aid in identifying the species of the sea urchin.
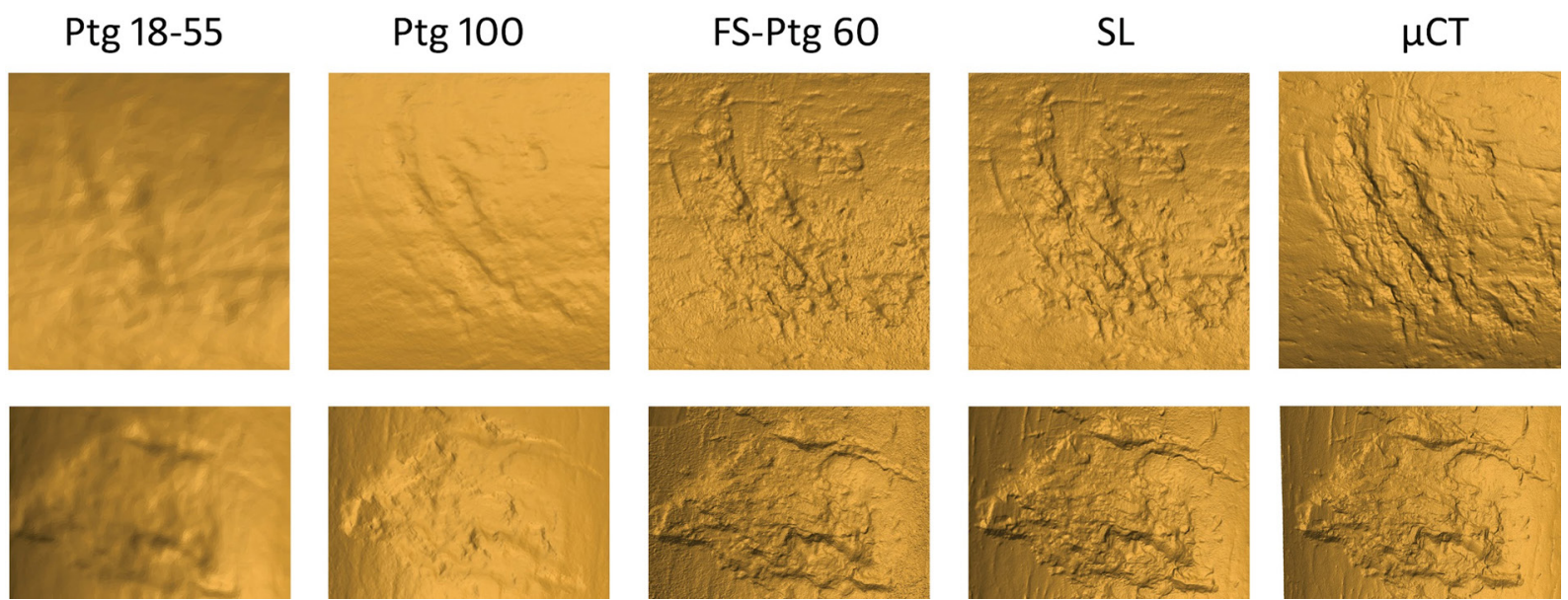

Fig. 6.7. Bone retoucher digitised with photogrammetry using a zoom lens (Ptg 18-55), a fixed focal macro lens (Ptg 100), focus stacking photogrammetry (FS-Ptg 60), structured light (SL) and microCT $(\mu \mathrm{CT})$. 


\subsection{Small specimens}

A beetle of the species Omorgus gigas is the test specimen used for the comparison of the 3D digitisation techniques for small specimens of $2 \mathrm{~cm}$ or less in size. The specimen belongs to the RBINS collection and is chosen because it is matte in appearance, which makes it easy to digitise for all the tested techniques. The comparison is only done with the dataset gathered with the RBINS focus stacking SfM setup. However, it is expected that the results will improve when using the DISC3D setup. The challenging parts in the morphology of the beetle are the different levels of fine structures. This gives the opportunity to judge which technique is able to capture all the details present and which one has limitations. The techniques that are compared to each other are focus stacking SfM, regular SfM, structured light scanning (MechScan) and $\mu \mathrm{CT}$ scanning. Although $\mu \mathrm{CT}$ scanning is not included in the list of possible $3 \mathrm{D}$ techniques in this volume, it is included in the comparison as it is probably one of the most accurate ways of digitising small specimens in 3D. To learn more about this technique and all of its possibilities for digitising natural history collections, see the dedicated volume of best practice for $\mu \mathrm{CT}$ scanning by Keklikoglou et al. (2019).

The $\mu \mathrm{CT}$ model will be considered as the best model that can be obtained. The other models will firstly be compared with this model and afterwards with each other. Looking at the $\mu \mathrm{Ct}$ model, it is clear that the main issue with scanning pinned insects are the artefacts created by the metal of the pin. The reason why is explained in the $\mu \mathrm{CT}$ volume of the best practice handbooks. A part of the information affected by the artefacts is lost and can be cut out to some degree in a fast but destructive way or in a more conservative way through carefully segmenting the area (Fig. 6.8).

The artefact caused by the metal pin, present in the uncleaned $\mu \mathrm{CT}$ model, is the main difference compared to the 3D models of the other techniques (Figs 6.9-6.11). But when looking at the other regions, unaffected by the artefact, different dissimilarities become apparent.

The MechScan model (Fig. 6.9) is least precise as it lacks most of the abdomen. This is caused by the inability of the camera to see the missing part by both cameras at the same time. Therefore, a complex object like a beetle at a size of approximately $2 \mathrm{~cm}$ is at the limit of the structured light scanner. If the

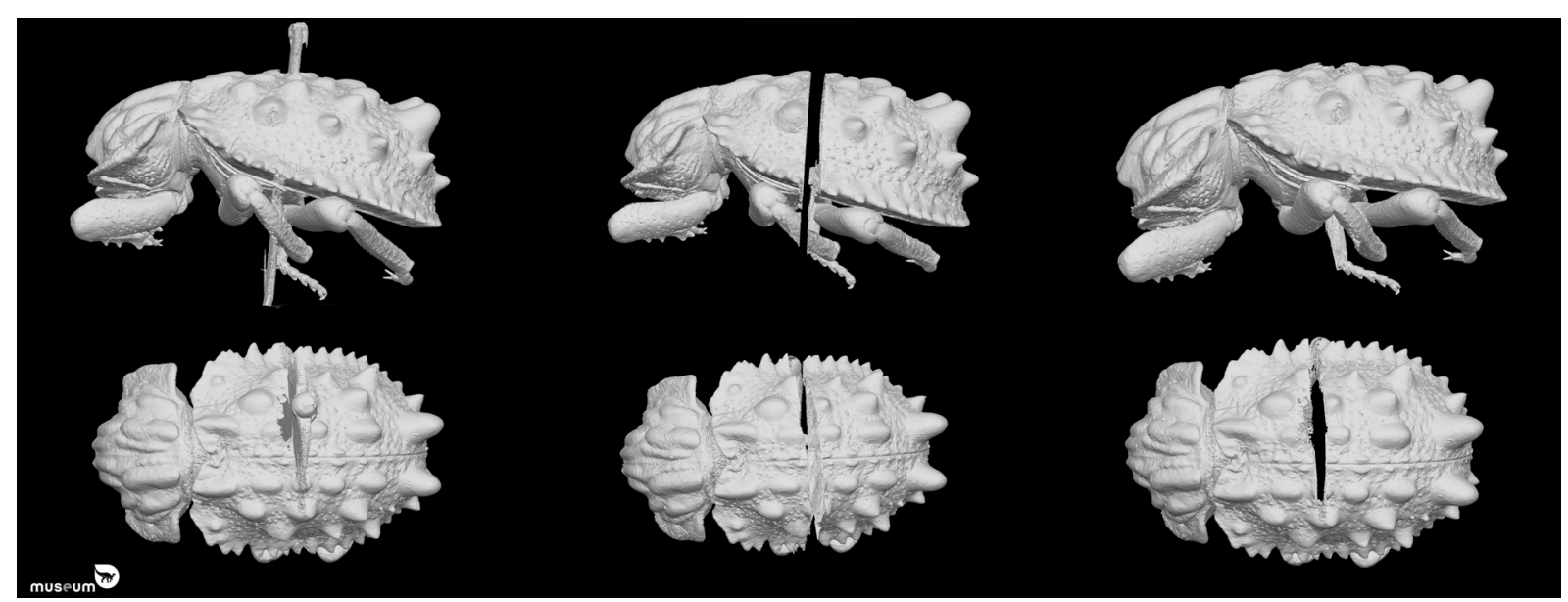

Fig. 6.8. Comparison between different treatments of the $\mu \mathrm{CT}$ model. Top and bottom left: $\mu \mathrm{CT}$ model without any treatment regarding removal of the artefacts https://sketchfab.com/models/169c2b29a1404e5cb5f6b6ea0928d075. Top and bottom in the middle: area affected by the metal artefact quickly cut out in GOM Inspect https://sketchfab.com/models/ f40a108e43ea44d78ca3d04ac41ad0a0; Top and bottom right: Carefully removed the needle through segmentation in Dragonfly 3.5. https://sketchfab.com/models/dc194a4e05da4f7ab787c8c7b028d2e7 


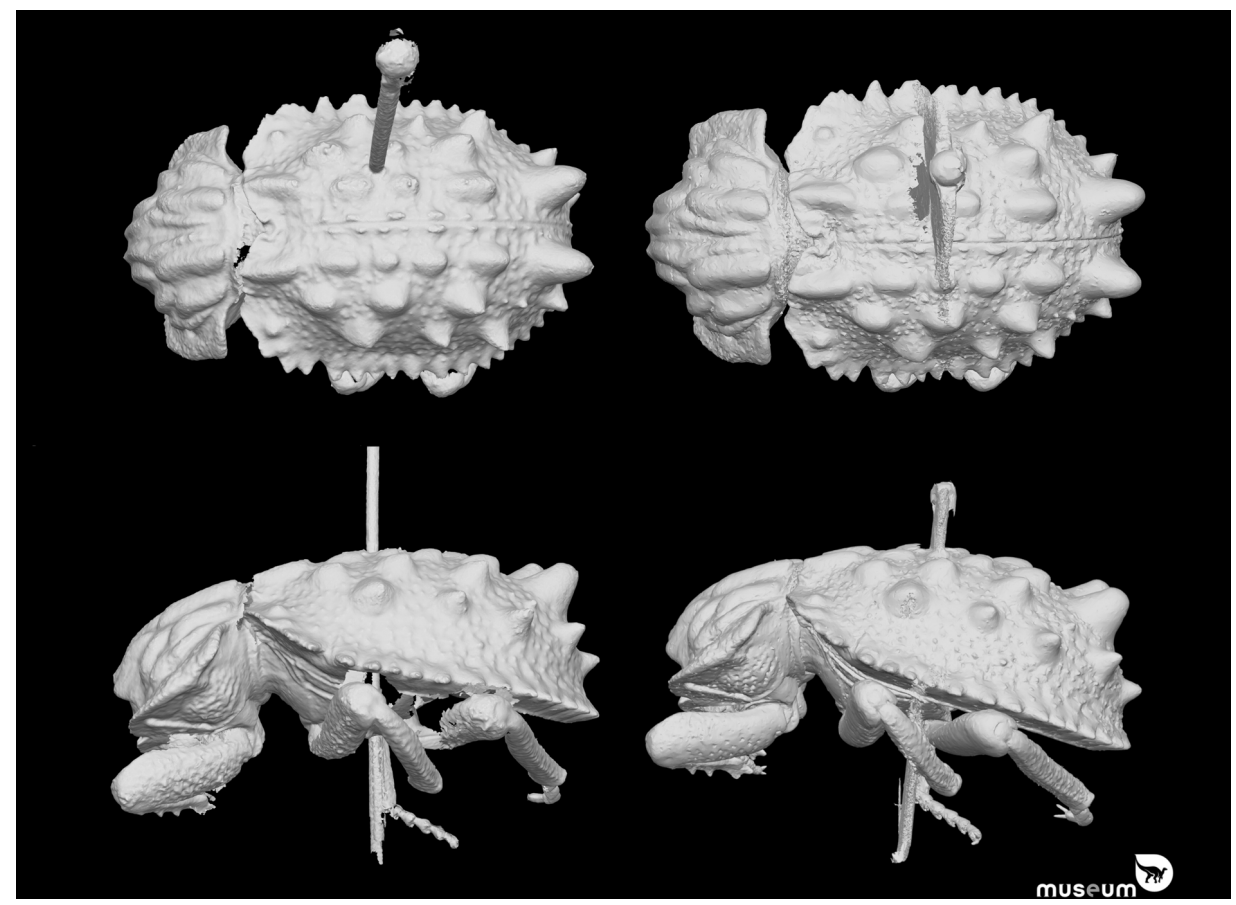

Fig. 6.9. Omorgus beetle structured light scanning with MechScan (left) comparison to $\mu \mathrm{CT}$ scan (right). The main difference is the lack of artefacts due to the needle with the MechScan 3D model. But the MechScan model lacks almost the entire ventral area of the abdomen between the legs. https://sketchfab.com/models/9dc5082c8db14c92879947da927f07dc

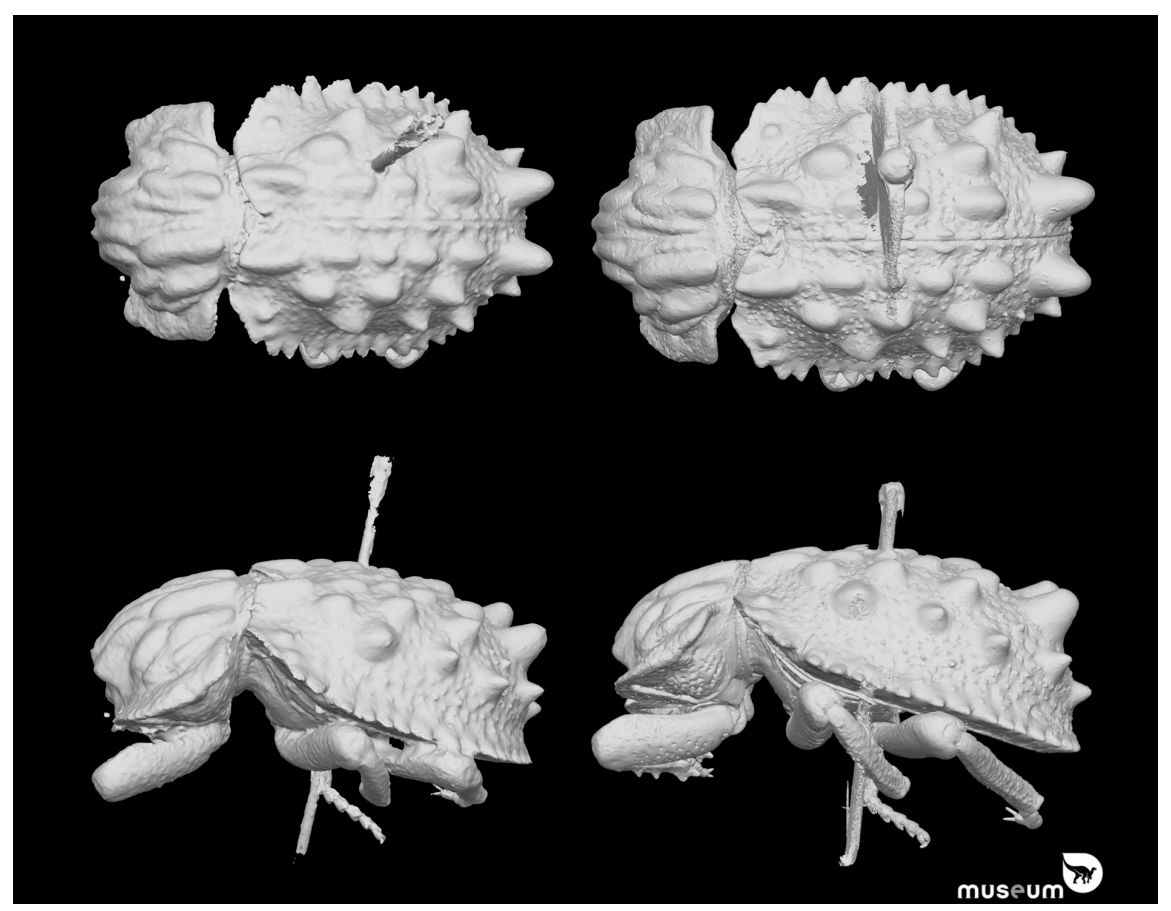

Fig. 6.10. Comparison of the Photogrammetry (left) vs $\mu \mathrm{CT}$ models (right) of the Omorgus beetle. The large difference between them is the less pronounced detail in the photogrammetry model. To fully see the detailed differences, check the models online:

https://sketchfab.com/models/a1fb6b8289f74c428782f43d502e771e 
object would be simpler and all the sides can be visualised by both cameras at the same time, there would not be an issue as is clearly visible by the details on the elytra.

The SfM and focus stacking SfM models resemble each other very well (Figs 6.10-6.11). The fine details and edges of the elytra are a little bit better for the focus stacking SfM model, but it is still very subtle. The reason for the success of the model obtained by regular SfM is that it is just within the range of what is possible without using focus stacking. As discussed in the SfM chapter for small specimens, it is clear that below $1 \mathrm{~cm}$, regular SfM is not capable of producing models without a high number of errors.

To conclude, it is clear that for small specimens only (focus stacking) SfM and $\mu \mathrm{CT}$ are a possible solution to digitise them in 3D.

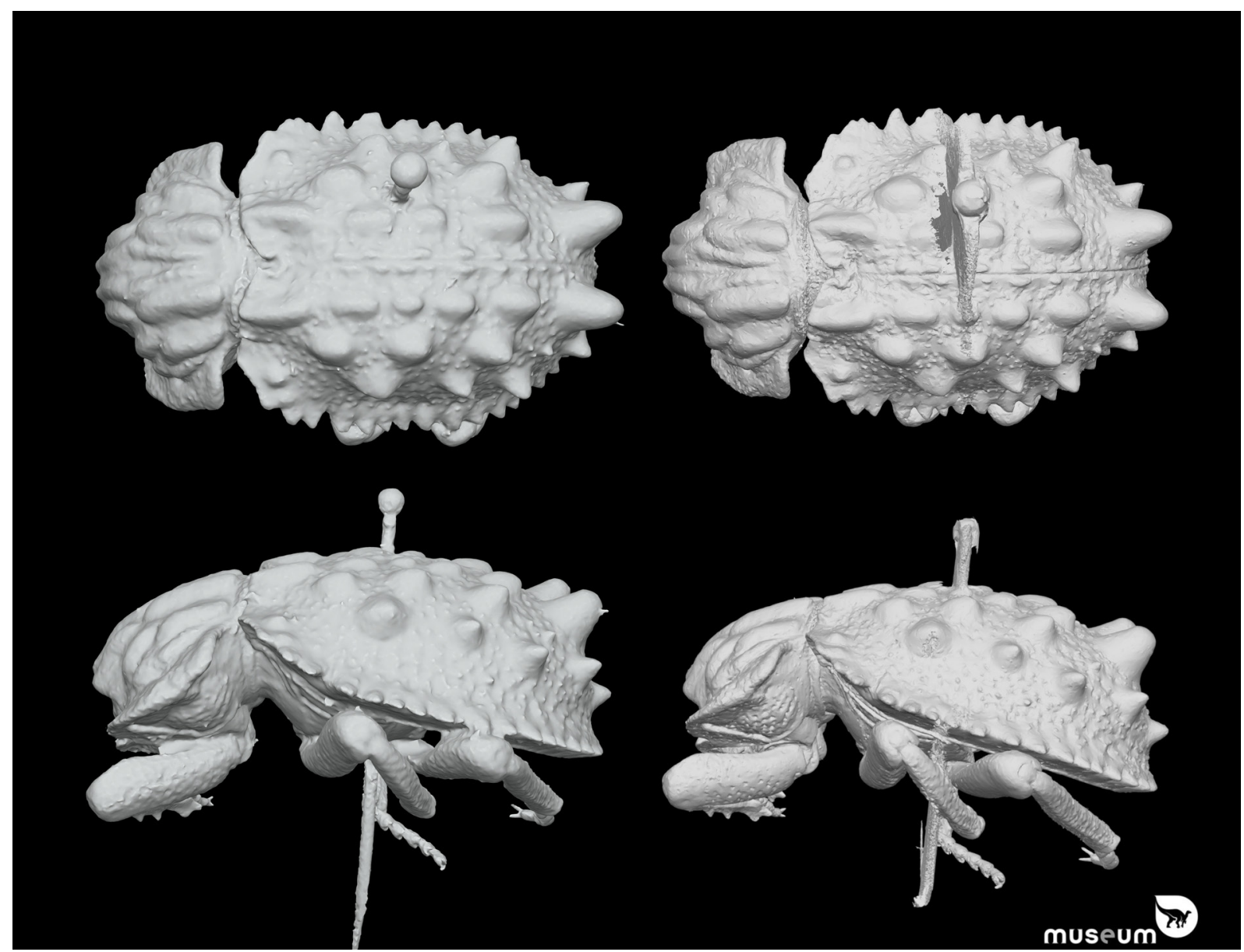

Fig. 6.11. Comparison between the Focus Stacking Photogrammetry model (left) and the $\mu \mathrm{CT}$ model (right) of the Omorgus beetle. The models are very close, with the $\mu \mathrm{CT}$ model perhaps showing a little more detail. The $\mu \mathrm{CT}$ scan was made using a XRE UniTom at $60 \mathrm{kV}$ and with a voxelsize of $29 \mu \mathrm{m}$. https://sketchfab.com/models/ad5fd157c5424c8fb793c1c282450d33 


\subsection{Cost of digitisation}

Another parameter to consider when choosing an equipment for digitising the collections is the cost/ efficiency ratio. If the aim is occasional digitisation, choice will be oriented toward a low-cost equipment where efficiency is not essential. If the goal is to digitise a complete collection it is better to choose more efficient equipment. Figs 6.12-6.13 show the cost/efficiency ratio of different techniques in function of the number of specimens/objects to digitise. A technique like structured light scanning that requires more investment at the beginning can turn out to be the cheaper option in the long term if many objects need to be digitised. This is due to the permanent cost of an operator. And often cheaper techniques require more time for an operator during and after scanning. Most of the more expensive techniques can be semi-automated, reducing the human time investment and lowering the costs in larger digitisation projects.

\subsection{Challenging materials}

Cost/efficiency is not the only parameter to consider when choosing a digitising technique: the material and its optical properties can also have an influence.

Indeed, not every scanning technique copes well with every kind of material. Some techniques are allrounders, generalists, and can perform well for almost every kind of material. However, the 'Jack of all trades is a master of none' theory fits here as well. SfM will be able to produce a good or decent result for almost every material type, but often another technique like structured light or laser scanning will perform equally well or better especially when scanning dark or homogeneous materials. However, these latter techniques lose the battle with SfM when it comes to versatility in different environments, quality of the texture, price of the system, ease of use, etc.

Laser scanner, structured light or infrared sensors struggle to record reflective or transparent materials as well as highly absorbing materials (Alhwarin et al. 2014; Koch et al. 2017; Pöhlmann et al. 2016). Infrared sensors simply do not see transparent material. One can easily scan an object directly through its glass case.

Each technique has its advantage and drawbacks and sometime the only way to have a good model is to combine different techniques. It is especially the case for objects made up by different challenging parts, that are dark, white, shiny, translucent, etc. if also a good texture is necessary, then the only way to digitise it would be to combine 3D models of different techniques together. This was the case for the Ishango rod (Fig. 6.14) that was digitised with a $\mu \mathrm{CT}$ for the quartz and the MechScan structured light scanner for the bone as a last step the SfM texture was applied as well. The crystal on top is perfectly modelled in the $\mu \mathrm{CT}$ scan, however the midsection of the bone shows a lot of artefacts and hardly any detail as it was an old $\mu \mathrm{CT}$ scan. The 3D model created by the MechScan structured light scanner has an almost perfect 3D model of the bone part but did not manage to capture the crystal on the top. In the end the texture SfM model was applied to the combined model of $\mu \mathrm{CT}$ and structured light to have a perfect 3D model for visualisation and research (https://sketchfab.com/models/8e296dfcabfc4161bad7005049b05676).

Of course, there are also other solutions to cope with challenging materials, as most 3D digitisation methods (with the exception of $\mathrm{CT} / \mu \mathrm{CT}$ ) have issues with reflective or transparent materials (RiekeZapp \& Royo 2017; Trucco et al. 1994; Alhwarin et al. 2014; Pöhlmann et al. 2016; Malisson \& Wings 2014; Mathys et al. 2015). One of the most common options in order to improve a 3D model is the use of coatings that increase the matte texture of the object (Busby 2016; Valinasab et al. 2015; Wachowiak \& Karas 2009). Many different options are available (developer spray, anti-glare spray, dulling spray, chalk spray, cyclododecane spray, talc dust, paint, etc.). But they can present a risk for the collections due to the removing process. Some can only be removed by water, other by friction and some can dissolve after a certain amount of time. There is also the risk of leaving a chemical trace onto the specimen. In addition 


\section{Cost of digitisation per specimen for medium sized specimens}

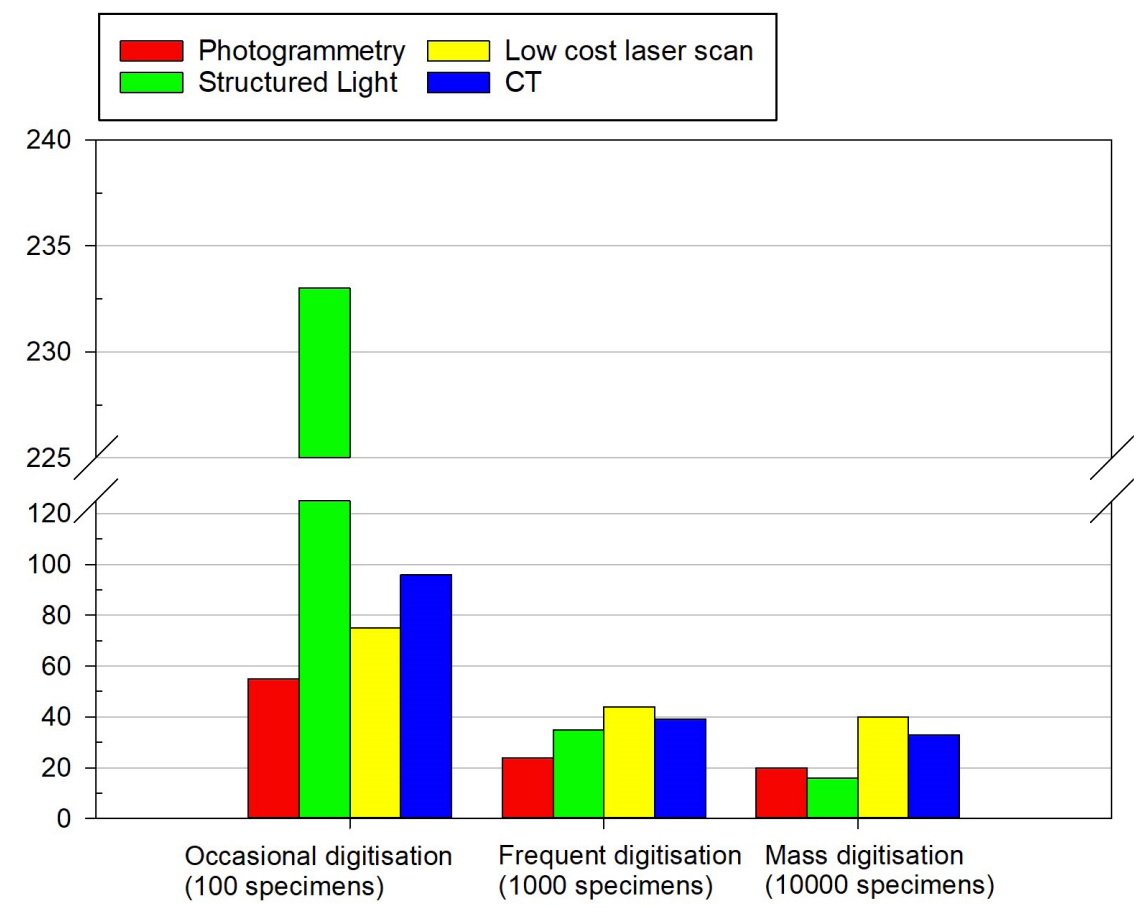

Fig. 6.12. Cost/efficiency ratio of digitisation for middle sized specimens. (CT is based on a rental service, while the others include the purchase of the equipment).

\section{Cost of digitisation per specimen for small sized specimens}

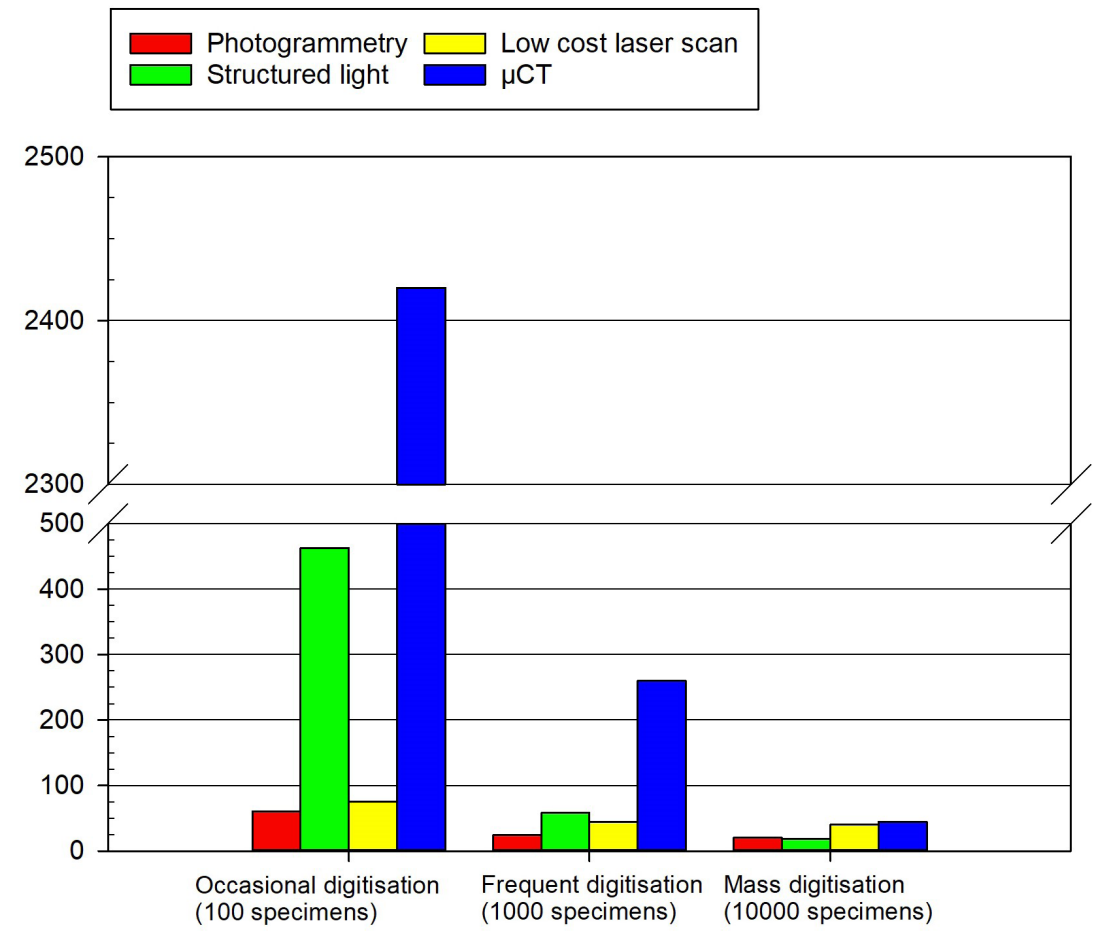

Fig. 6.13. Cost/efficiency ratio of digitisation for small-sized specimens. 
to that, the presence of these chemicals has to be taken into account as they can interact with the object. Porter et al. (2016) showed in their paper comparing different coatings for SfM and structured light that some coatings contain acetone, and this can dissolve the inventory number on the artefact. They also showed that coatings suitable for structured light and SfM are not necessarily the same.

Therefore, it is advised to digitise a collection without the use of coatings. Many tips and tricks exist regarding dealing with challenging materials in digitisation (Mathys et al. 2015).

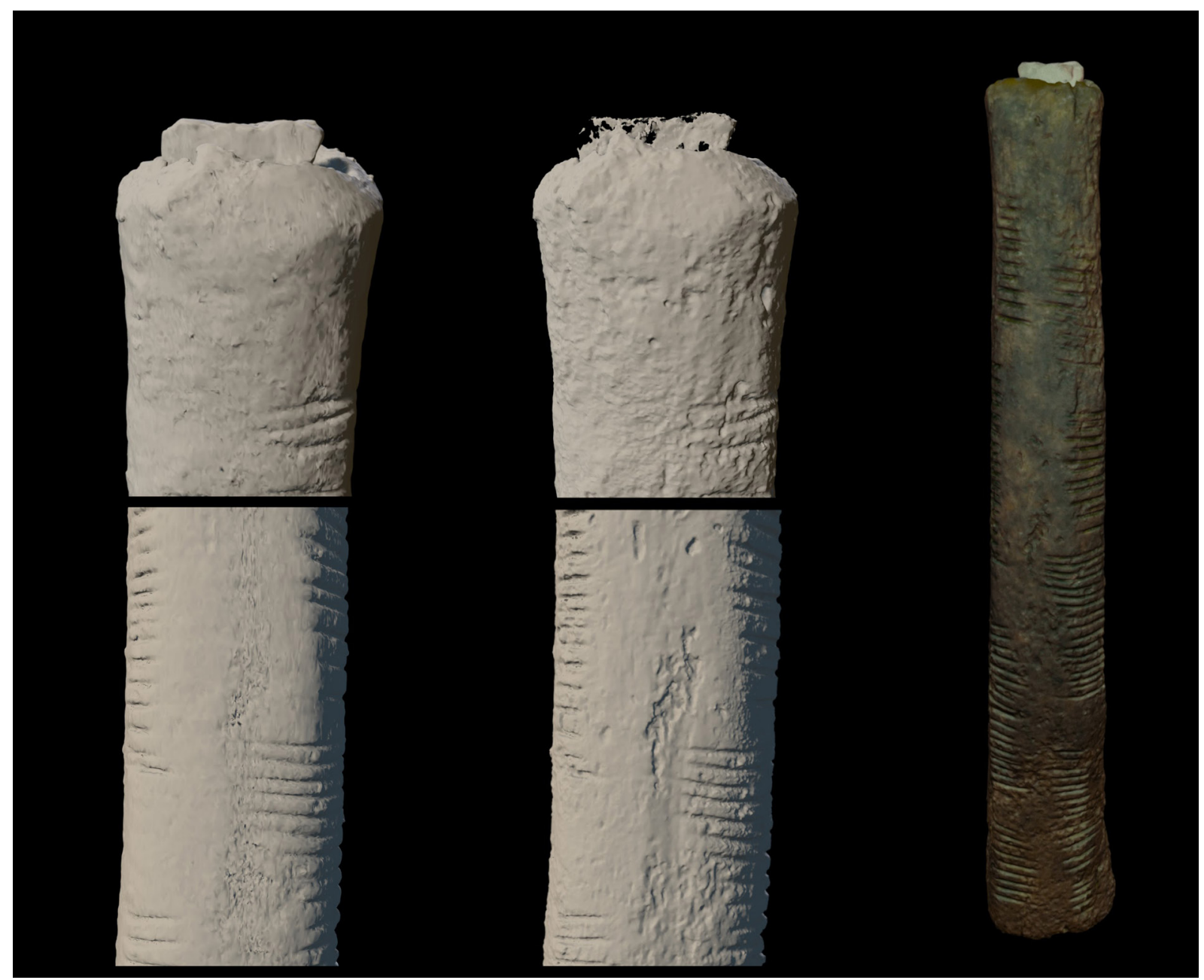

Fig. 6.14. Ishango rod. The left $3 \mathrm{D}$ model was acquired with a $\mu \mathrm{CT}$ many years ago. The middle one is scanned with the MechScan structured light scanner. The right one is the combination of both the $\mu \mathrm{CT}$ scan, the structured light scan and the texture of the photogrammetry model.

\subsection{References}

Alhwarin F., Ferrein A. \& Scholl I. 2014. IR Stereo Kinect: Improving Depth Images by Combining Structured Light with IR Stereo. In: Pham D.N. \& Park S.B. (eds) Lecture Notes in Computer Science Vol. 8862: PRICAI 2014: Trends in Artificial Intelligence. Springer, Cham. https://doi.org/10.1007/978-3-319-13560-1_33

Busby J. 3D scanning reflective objects with photogrammetry. 3D Scanstore. 2016. Available from http://www.3dscanstore.com/index.php?route=journal2/blog/post\&journal_blog_post_id=19 [accessed 5 Sep. 2018] 
Keklikoglou K., Faulwetter S., Chatzinikolaou E., Wils P., Brecko J., Kvacek J., Metscher B. \& Arvanitidis C. 2019. Micro-computed tomography for natural history specimens: a handbook of best practice protocols. European Journal of Taxonomy 522. https://doi.org/10.5852/ejt.2019.522

Koch R., May S. \& Nüchter A. 2017. Detection and purging of specular reflective and transparent object influences in 3D range measurements. ISPRS - International Archives of the Photogrammetry, Remote Sensing and Spatial Information Sciences, XLII-2/W3: 377-384. https://doi.org/10.5194/isprs-archives-XLII-2-W3-377-2017

Mallison H. \& Wings O. 2014. Photogrammetry in paleontology. A practical guide. Journal of Paleontological Techniques 12: 1-31.

Mathys A., Brecko J., Van den Spiegel D. \& Semal P. 2015. 3D and challenging Materials: guidelines for different $3 \mathrm{D}$ digitisation methods for museum collections with varying material optical properties. Proceedings of the $2^{\text {nd }}$ International Congress on Digital Heritage.

https://doi.org/10.1109/DigitalHeritage.2015.7413827

Pöhlmann S.T.L., Harkness E.F., Taylor C.J. \& Astley S.M. 2016. Evaluation of Kinect 3D Sensor for healthcare imaging. Journal of Medical and Biological Engineering 36 (6): 857-870.

https://doi.org/10.1007/s40846-016-0184-2

Porter S.T, Missal K. \& Pawlowicz L. 2016. A comparison of methods for creating 3D models of obsidian artifacts. Computer Applications and Quantitative Methods in Archaeology (CAA). Oslo, Norway, $29 \mathrm{Mar}$ -2 Apr. 2016. Presentation. Available from https://www.researchgate.net/publication/299820517_A_ Comparison_of_Methods_for_Creating_3D_Models_of_Obsidian_Artifacts [accessed 9 Jul. 2018].

Rieke-Zapp D. \& Royo S. 2017. Structured Light 3D Scanning. In: Bentkowska-Kafel A., MacDonald L. (eds) Digital Techniques for Documenting and Preserving Cultural Heritage: 247-251. Kalamazoo and Bradford: Arc Humanities Press, Amsterdam.

Trucco E, Fisher R.B. \& Fitzgibbon A.W. 1994. Direct calibration and data consistency in 3-D Laser Scanning. Proceedings of the Conference on British Machine Vision 2: 489-498. https://doi.org/10.5244/C.8.48

Valinasab B., Rukosuyev M., Lee J. \& Jun M.B.G. 2015. Atomization-based spray coating for improved 3D scanning. Journal of The Korean Society of Manufacturing Technology Engineers 24: 23-30. https://doi.org/10.7735/ksmte.2015.24.1.023

Wachowiak M.J. \& Karas B.V. 2009. 3D scanning and replication for museum and cultural heritage applications. Journal of the American Institute for Conservation 48 (2): 141-158. https://doi.org/10.1179/019713609804516992 


\section{Data management}

To manage the produced data, it is advised to keep track of several parts of the digitisation process. It is suggested that during the digitisation of an object or specimen a file is made of the digitisation process which answers the How?, When?, Which?, etc. questions.

Once a digital counterpart of the object is created it has to be saved on a drive of the computer and an external drive or cloud. Once every week or month this external drive has to be cloned or transferred to a server or long-term preservation platform. Once many specimens are digitised and it is known to researchers what the possibilities are with these digital specimens, digitisation on demand will become the standard. The data created has to be curated too, because they represent the specimen in a digital format. It is therefore extremely important that both the user and the creator of the data know what is possible with the data and what is not (cfr Creative Commons). Especially metadata of the created 3D data is key that all actions and post treatments and used software to obtain the final model are written down. These will play a crucial role when the digital specimen will be accessed to perform detailed research. One must not forget to keep the raw data safe so future recalculations of the original data is possible in case better algorithms are available.

It is very difficult to provide a 'one fits all' type of data management guide as the data and metadata created differ for each digitisation technique and not always the same post-processing actions are required or performed for each digitised specimen. But it is key that every action performed on the 3D model is traceable. Perhaps it is wise to use codes or abbreviations in the file name so it is immediately clear which (post)-processing actions have been performed.

In case more information on the subject is needed the following outcomes on a European level of the SYNTHESYS 3 project can be consulted: http://synthesys3.myspecies. info/node/596, http://synthesys3.myspecies.info/node/698, http://synthesys3.myspecies.info/node/ 671 and http://synthesys3.myspecies.info/node/695. The information listed by iDigBio, funded by the US National Science Foundation is also pertinent: https://www.idigbio.org/wiki/index.php/Digitization_Resources and https://www.idigbio.org/portal/ search. 


\section{Data distribution}

Digitisation of museum objects and specimens for the sake of having a virtual backup might be one reason to do this, but another important reason is making these copies available to other researchers and perhaps even the general public.

Considering the size of the files and the storage capacity needed not only to store, but to make the digital twins accessible, Sketchfab, although a private company, for now is the easiest and cheapest solution to host the institutional 3D files (Fig. 8.1). Sketchfab offers reduced Premium accounts to institutions and

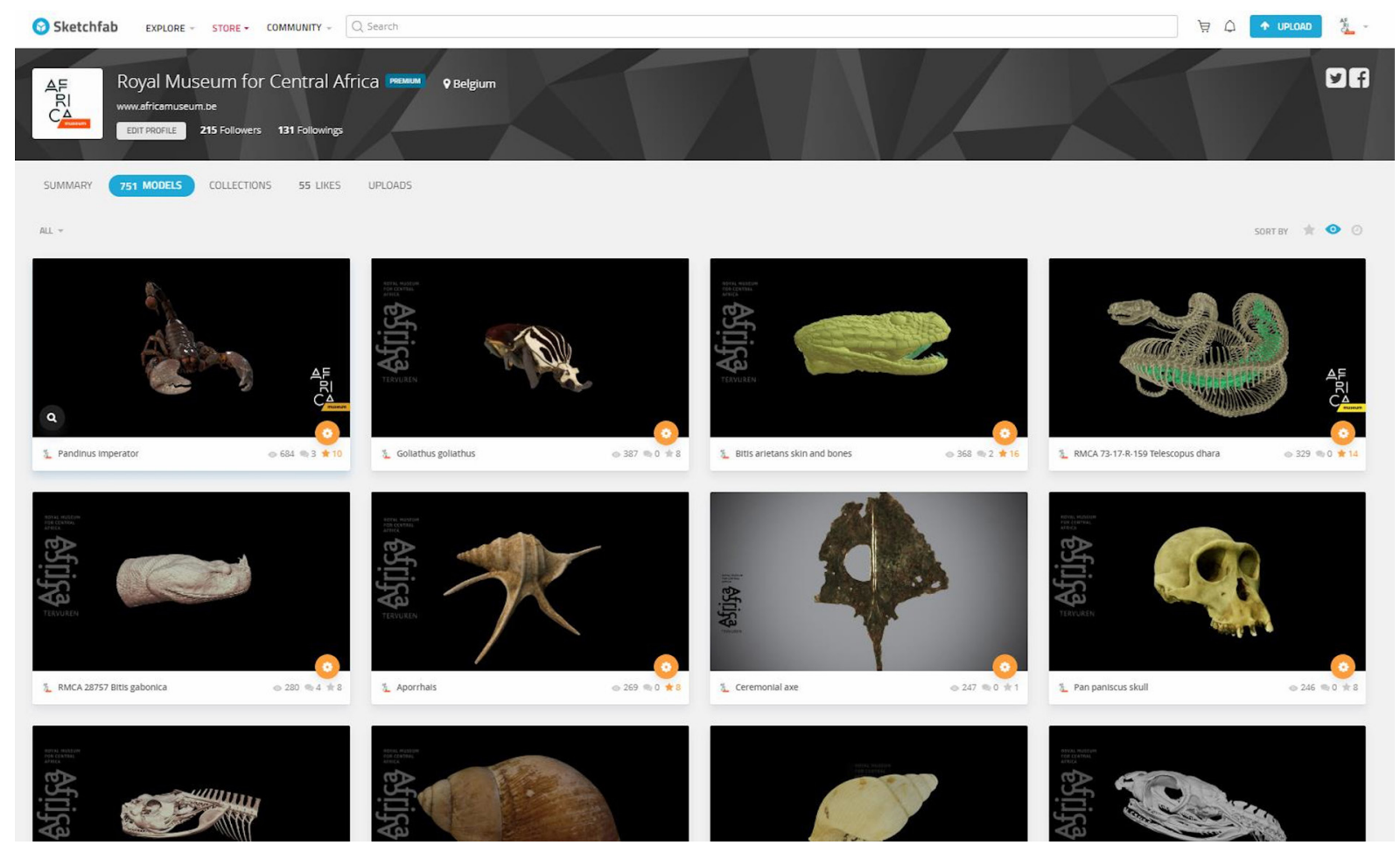

Fig. 8.1. Sketchfab page of RMCA.

research facilities and are very keen on developing new tools which can aid the research on the models. Important to note is that in a premium-account, it is possible to place models in private folders. Premium accounts are limited to 200 private model uploads a month and 50 annotations per model (Fig. 8.2). And maybe the most important thing is that the one uploading the files, keeps the copyright of them.

It would be better if a similar repository is created on a European (like the DISSCo consortium https://www.dissco.eu/) or Global scale specifically for research institutions. There are some alternatives like 3DHop (http://vcg.isti.cnr.it/3dhop/), but it requires proper server space hosted by the research facility or consortium itself. In case of a general research repository it could be further adapted so performing simple measurements (size, surface area, volume, ...) or analysing (geometric morphometrics, model comparison, ...) the 3D models would be possible. In this way the collections are really opened to any researcher.

RBINS and RMCA, like many other institutions, also developed their own online platform for sharing the digitised specimens. At RBINS this platform is called Virtual Collections (http://virtualcollections.naturalsciences.be/) and Collections (http://collections.naturalsciences.be/) 


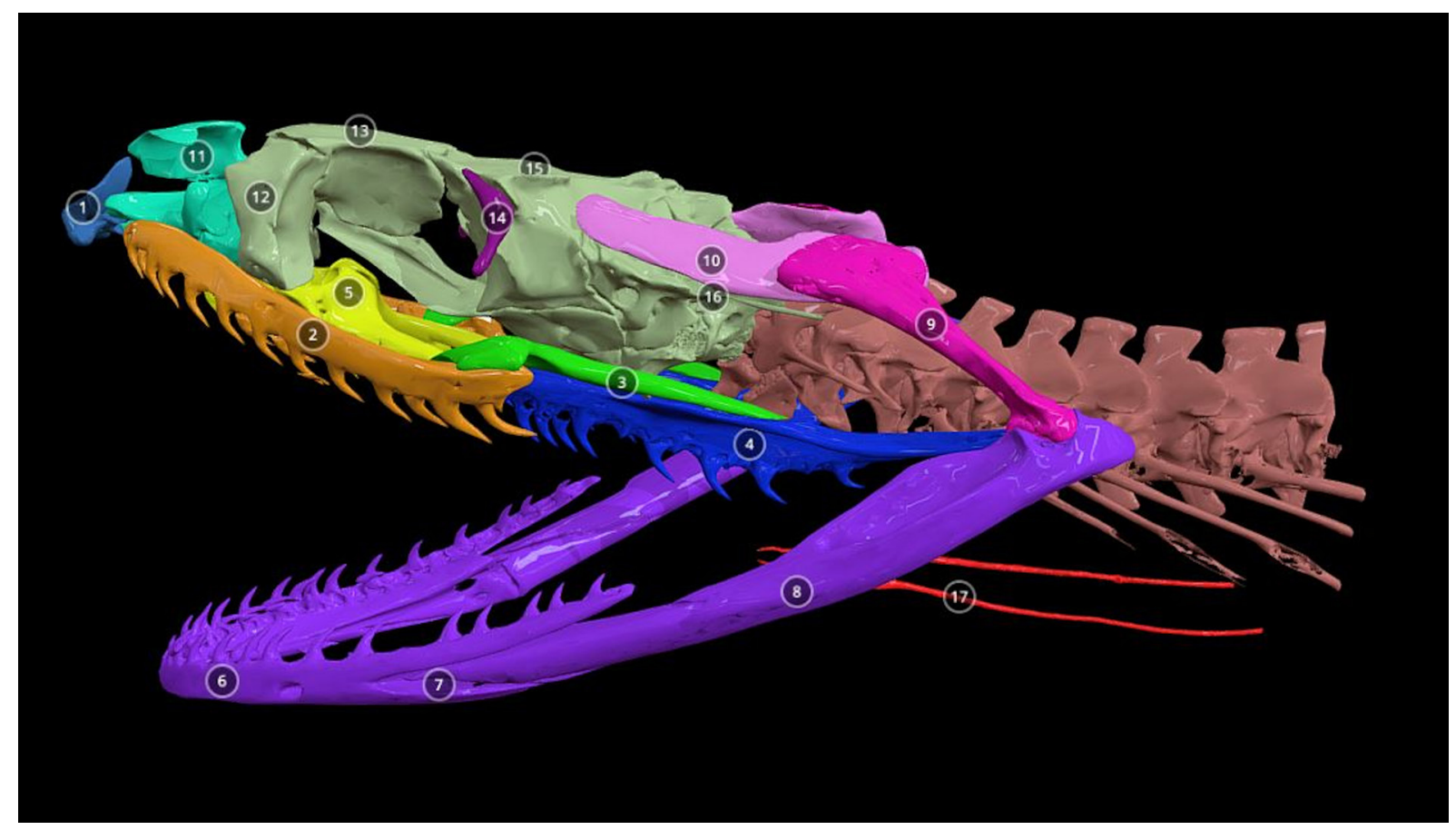

Fig. 8.2. Annotated model of a grass snake skull on Sketchfab. https://skfb.ly/6tNwY

\section{museum}
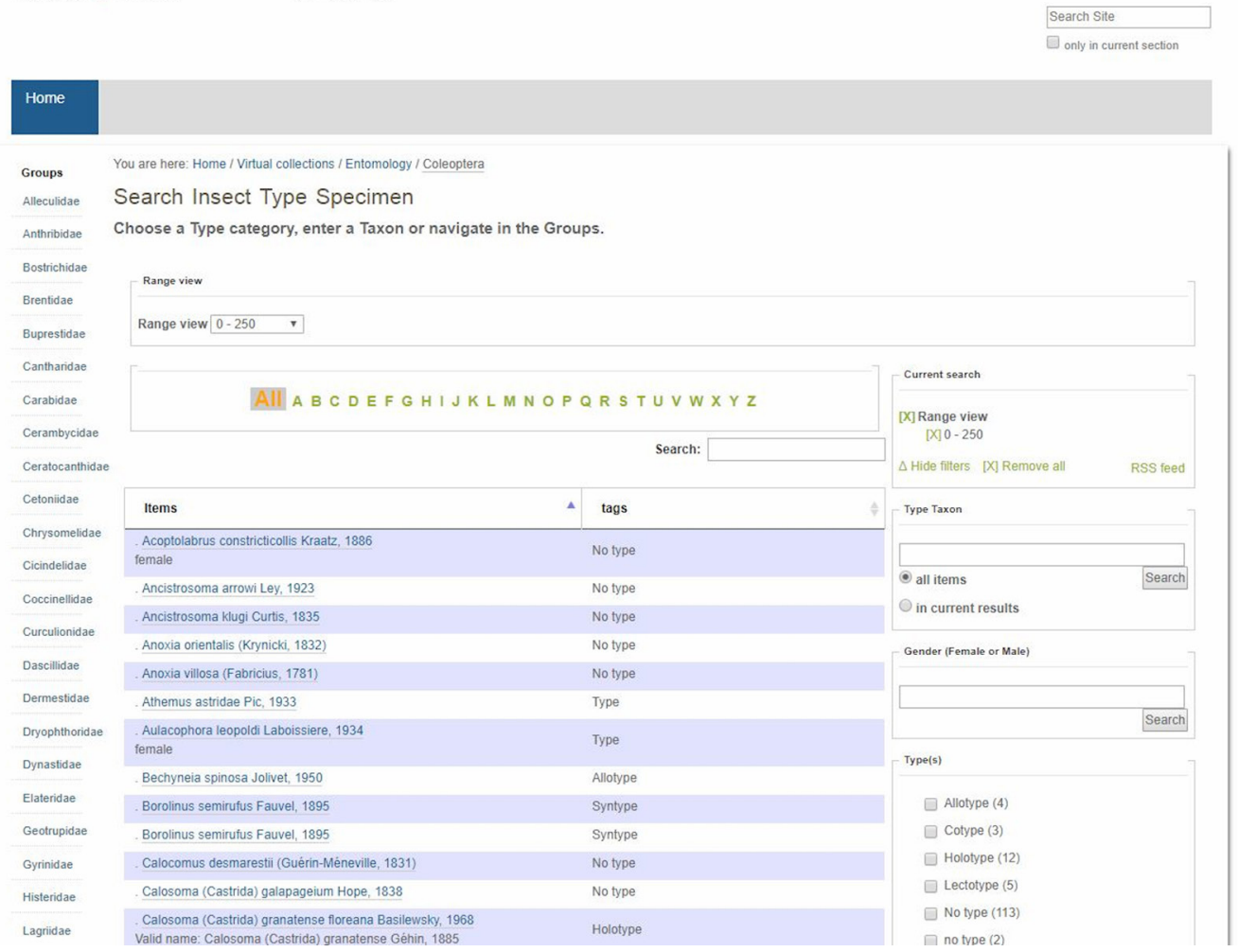

Fig. 8.3. Virtual collections page of RBINS 


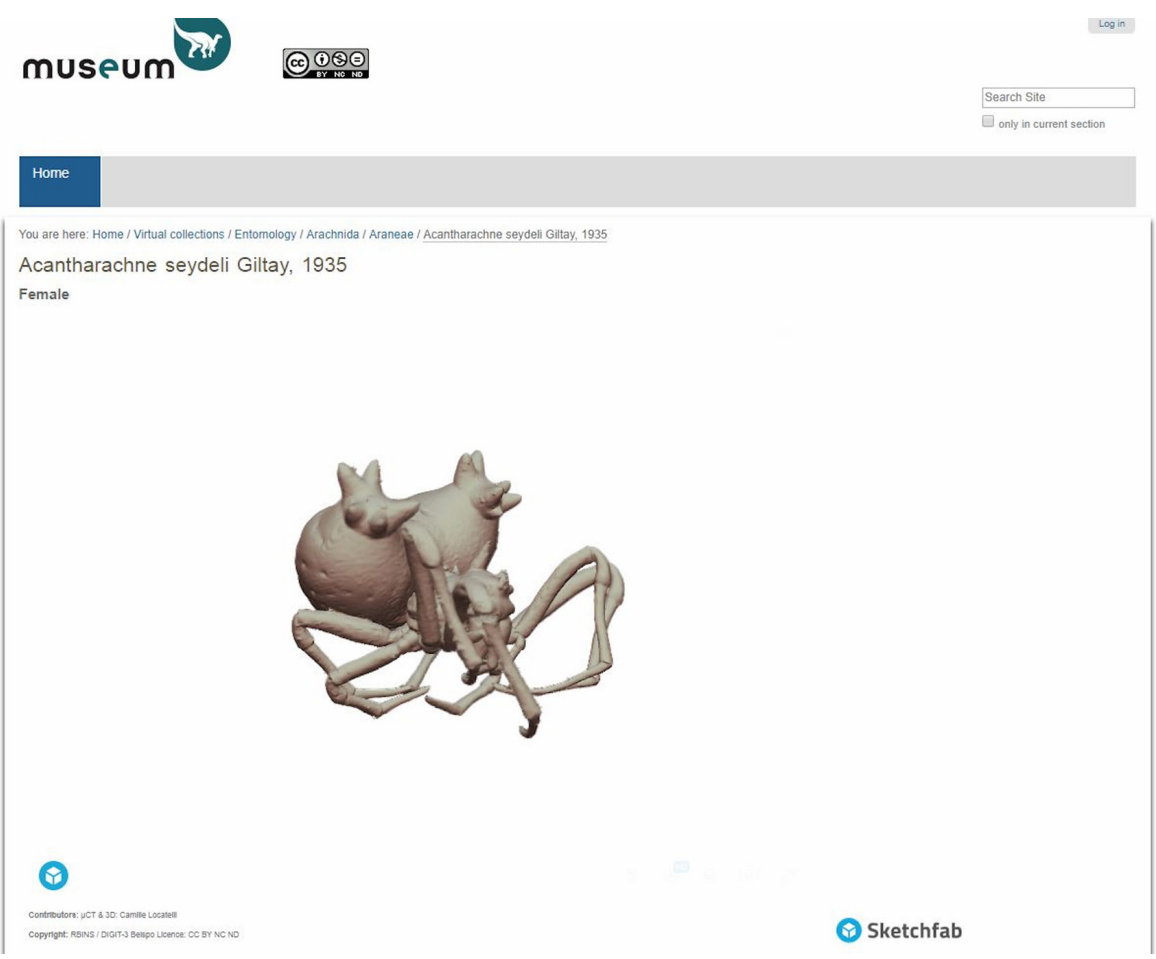

Fig. 8.4. Virtual collections page of RBINS: Specimen view.

(Figs 8.3-8.4). The digitised objects or specimens are available in the different collections. All the specimens are linked to the catalog database DaRWIN (http://darwin.naturalsciences.be/), making the pictures and the data accessible to anyone. At RMCA the platform is called Digit03

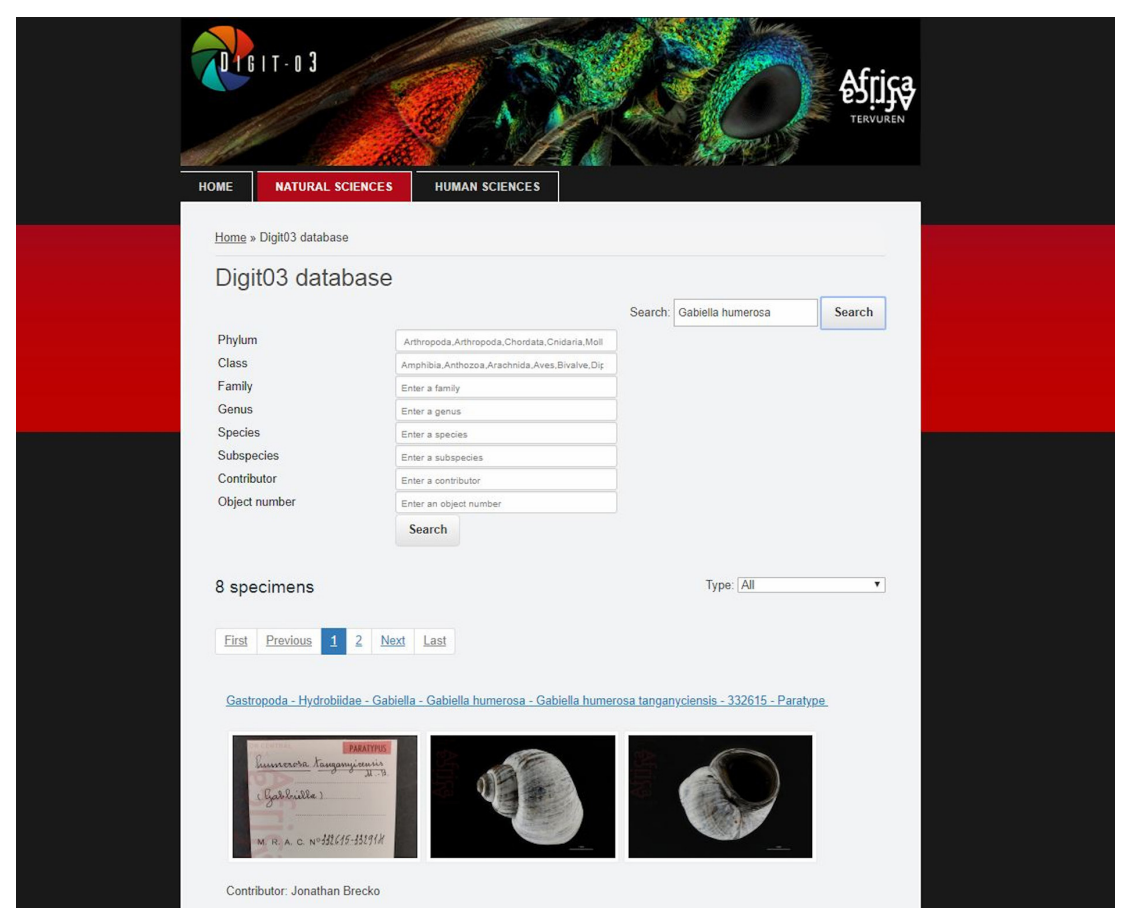

Fig. 8.5. RMCA Digit03 dissemination platform. 
(http://digit03.africamuseum.be/) (Fig. 8.5) host the digitised object and specimens. Both platforms include a search engine.

In case more information on the subject is needed the following outcomes on a European level of the Synthesys3 project can be consulted: http://synthesys3.myspecies.info/node/596, http://synthesys3.myspecies.info/node/698, http://synthesys3.myspecies.info/node/671 and http://synth esys3.myspecies.info/node/695. The information listed by iDigBio, funded by the US National Science Foundation is also worthwhile to have a look: https:/www.idigbio.org/wiki/index.php/ Digitisation_Resources and https:/www.idigbio.org/portal/search. Or the Australian digitisation initiative by CSIRO (Commonwealth Scientific and Industrial Research Organisation), https://www.csiro. au/en/Research/Collections/Digitisation, https://www.ala.org.au/. 


\section{Workflows}

\subsection{Workflow 2D+Focus Stacking DSLR@RBINS and RMCA}

\section{Taking the pictures}

Visualise live on screen using the software of your camera. There are a few things one needs to consider in order to take pictures for focus stacking.

In order to make sure that the specimen is in focus during the picturing process and that it in the desired position, one can use the live view mode of the camera, set the shutter speed on the 'BULB' function and make sure the specimen is well lit.

First the magnification is adjusted according to the specimen size or part that one wants to picture.

Next, the top and the bottom parts of the specimen (or the part that needs to be in focus) are determined and if a motorized rail is used, these values should be stored in the software. The easiest way to determine the top and the bottom parts of the specimen is by moving the camera up until everything is blurred, then slowly moving it down again till the first part appears sharp. This is easier noticed performing it this way than starting at a point where parts are already in focus. The same method can be used to select the bottom part by lowering the camera until everything is blurred then slowly moving back up again.

Subsequently the shutter speed is chosen (1/100 s when using speed lights).

Afterwards the power of the speed lights is adjusted. If a magnifying lens is used, like the Canon MP-E $65 \mathrm{~mm}$, more light is needed when a higher magnification is used. This means the speed lights flash with more power and the batteries will need to be recharged quicker. To avoid this there are a few options:

- Reduce the volume of the chamber in which the specimen and the speed lights are in by blocking light paths where no light is needed or moving the speed lights closer to the specimen. In a cupboard it is easily done by moving the shelves of the specimen and the speed lights closer to each other.

- Use a higher ISO value. Check the amount of noise that is created when shooting at ISO100, ISO200, etc., to ascertain the maximum level acceptable.

- Buy external battery packs for the speed lights or look out for those models that can be plugged directly in the mains.

To determine the step size of the focus stack (f), it is important to know the depth of field (dof) at a given magnification and given aperture. This depth of field table is usually written down in the manual of the lens that's used. To give an idea, the values that work for RBINS and RMCA using the Canon $65 \mathrm{~mm}$ MP-E lens are as follows:

- $1 \times$ magnification and $300 \mu \mathrm{m}$ step size and $\mathrm{f} / 5.6$ or $\mathrm{f} / 6.3$

- $2 \times$ magnification and $200 \mu \mathrm{m}$ step size and $\mathrm{f} / 5.0$ or $\mathrm{f} / 5.6$

- $3 \times$ magnification and $150 \mu \mathrm{m}$ step size and $\mathrm{f} / 4.5$ or $\mathrm{f} / 5.0$

- $4 \times$ magnification and $75 \mu \mathrm{m}$ step size and $\mathrm{f} / 4.0$ or $\mathrm{f} / 4.5$

- $5 \times$ magnification and $50 \mu \mathrm{m}$ step size and $\mathrm{f} / 3.5$ or $\mathrm{f} / 4.0$

The reason for using the above aperture sizes at the different magnifications is because of the effective f-number of the lens at the specific magnification. It is best to keep these in the optimal range, which is mostly $\mathrm{f} / 5.6$ to $\mathrm{f} / 8$, but depends on the lens used. It is recommended to make some test images at different 
f-stops to see which value gives the best results. For the MP-E at $5 \times$ magnification an aperture of $\mathrm{f} / 2.8$ would theoretically be the best, but no differences were observed between an f/2.8 and f/4 aperture size.

If a scale needs to be added to the picture afterwards it is important to note down the magnification that is set while taking the pictures. But as previously mentioned, this is not for measuring purposes, just to have an approximate idea of the object size.

Normally a stack of $15-40$ pictures is created for the mean values. If the stack turns out to be a lot more, check if the settings are correct.

Some of the consumer DSLR cameras do not allow speed light to be controlled automatically by the camera when the live view window is still open. Therefore, it is important to remember to close this window if it is the case for the camera that is used.

\section{Stacking the pictures}

Numerous types of software exist to stack a set of pictures and extend the depth of field. Out of a test in 2014 (Brecko et al.), which is a long time ago considering software, both Helicon Focus and Zerene Stacker performed equally. The latter is used by both RBINS and RMCA, while the first is the software of choice for the MfN. Zerene Stacker performs very well when there is sample movement in the X-Y plane, which often occurs when shooting pictures of small specimens submerged in ethanol. Often it manages to stack the complete set without any noticeable defects.

When choosing the software to make the stacked pictures it is also important that it is possible to perform this process in a batch as at the end of the day many stacks are made and, in this way, the human interaction time is less. Another important feature should be that the name of the folder is copied in the name of the stacked picture. In this way it is always clear which specimen or part is represented on which picture.

\section{Making scales to add to focus stacked images}

Picture a microscope measuring slide with the camera at high magnification or a tape measure for images of larger-sized specimens.

Do this for all the magnifications that are used. To ease the work, it is also possible to calculate the number of pixels $1 \mathrm{~mm}$ represents using the following equation: $(\mathrm{A} / \mathrm{B})^{*} \mathrm{C}$ where $\mathrm{A}=$ magnification, $\mathrm{B}=$ sensor size $(\mathrm{mm})$ and $\mathrm{C}=$ image width (pixels)

For a magnification of $5 \times$ using a Canon EOS 700D (sensor size $22.3 \mathrm{~mm}$ ) and an image width of 5184 pixels, $1 \mathrm{~mm}$ is represented in the picture by 1162.3 pixels, which is the same value as measured using the microscope slide.

Once all the scales are made, one can start adding these to the focus stacked images using a batch program.

Positioning specimens in alcohol can be challenging from time to time; it is key to add just enough alcohol in the holder (petri dish, glass beaker...) to submerge the specimen. If too much is added, the specimens tend to float or move around while picturing.

To create a smooth background, it is important to leave enough distance between the specimen and the background. This can be done by placing the specimen onto a scratch-free transparent surface (glass or acrylic) which is suspended at a distance from the background. The easiest way is to use an upside-down petri dish or glass beaker. It is important that it is scratch-free (and dust-free), otherwise the background will look dirty. Taking pictures this way reduces the time needed for post-processing a picture or avoids it completely. 


\subsection{Workflow Microscope (Information in Imaging)@RBINS and RMCA}

Place the specimen on the slide holder and start by using the lowest magnification.

On a clean and clear surface, set the white balance and adjust the exposure. If possible, perform a shading correction too.

Moving the specimen in the $\mathrm{X}-\mathrm{Y}$ direction brings it into the field of view and focuses the microscope. Select the specimen by drawing a grid around the it, then perform a quick scan to locate the specimen. Afterwards the grid can be adjusted.

If a higher magnification is necessary to obtain a more detailed view of the specimen, change to another lens and determine the white balance, exposure and shading. Afterwards, the scanning process can be done again by selecting the specimen using the drawing grid function.

If the specimen is thicker than the depth of field, a Z-Stack is necessary. Prior to performing the scan, the upper and lower value together with the step size for the Z-Stack needs to be determined.

For best results, save each X-Y mosaic image and stack the different Z-Stacks in other software like Zerene Stacker afterwards.

\subsection{Workflow ZooSphere@MfN (by Susan Stephan)}

After choosing an object, a specific QR-code is allocated permitting the retrieval of the digital metadata afterwards. With a special reading device this QR-code can be scanned and filed along with its relative details.

\section{Mounting: harnessing and adjusting the object}

A mounting device is a gadget in which the object is clamped (left side of picture 2.17). To align the object at the appropriate altitude, the height adjustment is being used. The step-by-step workflow is as follows:

- Fix mounting device on desk plate.

- Place height adjustment reference next to mounting device.

- Open the pin's screw and adjust the needled object carefully on the pinhead, then tighten the screw again.

- Open the screw of the mounting device, so that the gap is large enough to hold the pin.

- Insert the pin into the centre of the gap of the mounting device and tighten the screw of the mounting device. Now the mounting device can be operated with one hand only.

- Carefully hold the object by the needle and adjust it with your free hand. The object should be placed perpendicular to the rotation axis. Rectify if necessary using a magnifying glass.

- Match object height with height adjustment reference.

- Tighten screw, so the pin is fixed in position.

- Find the furthest part of the object most remote from the needle (centre, pivot point); this crucial to guarantee all areas of the object are in focus (if it's difficult to distinguish the furthest point, use a ruler to help). This spot is your $1^{\text {st }}$ reference point.

- The object is rotated exactly $180^{\circ}$ to find the most distant part in this position. This is your $2^{\text {nd }}$ reference point. Your object will be photographed between reference points 1 and 2 .

- Open the back part of the ZooSphere cover. 
- Fit the mounting device onto the engine pin of the ZooSphere swing, and tighten the screw at the ring of the mounting device to the flat part of the engine pin.

- Make sure that the object is firmly clamped.

- Replace the back cover.

\section{Calibration}

- ZooSphere swing in "Null position" - swing right-angled to basis.

- Switch on ZooSphere, camera, Stackshot and flashlight device before you start the computer.

- Start the computer.

- Start the ZooSphere Encephalon Software.

- Connect to ZooSphere.

- Connect to camera.

- Connect to Stackshot.

- Hit the green "Play" button on the right of the toolbar to start the calibration manager.

- Choose a reasonable magnification of the macro lens. You may have to move the object to see if it fits into the image throughout the complete sequence.

- Put ZooSphere in horizontal position.

- Use the Stackshot controls to move the Stackshot and focus the object.

- Click "Calibration Position": check if ZooSphere swing is at a right angle to the base.

- Take an image and use the histogram to set an appropriate brightness of the flashlight device.

- To change the brightness of the flash device, use the arrow buttons next to the number display.

- To set start and end points of the focus stacking, click on the "Start Live View" button and turn on the "Lamp" function of the flashlight device to "Full" intensity.

- The $1^{\text {st }}$ and $2^{\text {nd }}$ reference points determined earlier in the mounting step are also the reference points used to calibrate the focus stacking start and end points.

- Move the ZooSphere swing to calibration position.

- Turn the object to the $1^{\text {st }}$ and most remote reference point of the object facing the camera.

- Adjust Stackshot until reference point is in focus.

- Move the focus point further away by moving the camera towards the object, by one or two steps, until the object is completely out of focus. This adds some buffer, in case you did not exactly match the best reference point for calibration.

- Press the "Set Focus Start" control.

- Turn the object by $180^{\circ}$ and focus the reference point again.

- Move the focus point further away from the camera, by one or two steps, so that the complete object is out of focus.

- Press the "Set focus end" button.

- Turn off the Live View and the lamp mode of the flashlight device. 
- Move object and swing as close to the camera as possible and make sure that the cover plate of the mounting device is not visible in the picture.

- Rotate the object to an appropriate starting position.

- Click the "Apply Calibration" button.

- Check the step size and adjust it according to the table, by either increasing or decreasing the number of stacking steps.

- Hit the "Start Sequence" button.

- Name the sequence.

- Choose the settings for the sequence recording process.

- Start the sequence.

\section{After sequence capturing}

- Remove the back cover of the ZooSphere device.

- Use the software interface to move the ZooSphere swing to the calibration position.

- Turn the motor the object is pinned on so that the screw at the ring of the mounting device faces you.

- Loosen the screw at the ring of the mounting device.

- Remove the mounting device carefully.

- Fix the mounting device in the desk plate.

- Grab the pinhead of the object.

- Loosen the main screw of the mounting device.

- Remove the object from the mounting device and place it into the box.

- Mount the measurement device onto the engine on the ZooSphere swing.

- Move the Stackshot to focus the scale image.

- Use the "Take Scaling Image" button to take an image of the scale.

- Copy all sequence files from the hard disk to the network location.

- Turn off all devices.

\subsection{WorkFlowRTI/PLD@RBINS}

For H-RTI an excellent workflow already exists: "RTI: Guide to Highlight Image Capture" by Cultural Heritage Imaging (http://culturalheritageimaging.org/What_We_Offer/Downloads/Capture/index.html).

The following short workflow is exclusively for PLD.

Before starting an acquisition, it is important to choose the proper lens according to the object that needs to be digitised. Fixed focal lenses that can be mounted on a machine vision camera are used.

It is best to place the dome in an area where no direct light interferes with the LED lights lit by the dome itself.

The specimen needs to be placed in the centre of the dome at a distance equal of the radius. Light all the lights of the dome to focus the lens. Once the specimen is in focus the calibration of the dome needs to 
be done by replacing the specimen by the calibration board and starting an acquisition with an exposure of $500 \mathrm{~ms}$. Afterwards, the specimen can be digitised, and a white balance correction can be done.

Each acquisition still needs to be processed. Once done it can be exported as a 3D model or measurements can be made and profiles may be drawn.

When processing the acquisition, it is important to use a Bayer Filter (GBRG) if the recording is done by a colour camera.

An important notice: the PLD recordings are not compatible with the RTI viewer and do not enable viewing RTI files processed with other software

\subsection{Workflow for close-range SfM@RBINS and RMCA}

The tutorial described is a general tutorial for SfM.

\section{Prepare the setup}

Centre the object on your turntable with a scale next to it. Try to have a smooth light (avoid using direct flash light) and uniform background. Position the camera, focus it and adjust the parameters (we usually work with an aperture between F11 and F18 depending on the shape of the object in order to have a good depth of field and a decently sharp image (F11 sharper, F18 more depth of field). This can change depending on the properties of the lens used). It is recommended to use macro lenses with fixed focal length for better results and to place the camera on a tripod, this will allow long exposure in poor light condition (it's better to have the ISO as low as possible).

Place a scale on the turntable for at least one of the rotations. It is also possible to use photogrammetric targets, this can help with the alignment, but the targets need to be different for each object position.

Either an automated turntable, like the AGORA 3D Automated photographer Foldio360 or similar setup, or a manual turntable can be used. The automated setup will naturally be faster than the manual setup, where the operator needs to rotate the turntable manually after each picture taken. If the object is too large to fit on a turntable, it is possible to take pictures of the object by circling around it. It is important that all pictures are taken from a different position relative to the object. So, for each view, the camera or the object has to move in relation to the other.

\section{Take the pictures}

For a standard medium-sized object, 4 rotations of 36 pictures are usually taken. To obtain these, start the application. If a manual setup is used with a camera mounted on a tripod, it is easier to work with a remote or the self-timer function of the camera.

Load the pictures on the computer in a convenient folder

\subsubsection{Workflow with a uniform background}

In several types of SfM software (Agisoft Metashape for RMCA and RBINS) it is possible to import both pictures of the specimen or object and a background picture. In the software itself the pictures can be masked automatically so only the information of the specimen will be calculated in in a 3D point cloud. The background picture is simply a picture without the specimen, it is best is to apply a Gaussian blur to it. Another way to do it is to select a small square of the background in photo processing software and expand this to the whole picture.

The most important next step is aligning the pictures. Make sure to carefully look at the alignment and delete or re-align pictures that are not in the correct position. 


\subsubsection{Workflow without a uniform background}

If the background is not uniform because many other objects are visible, for instance if a specimen is too large to put in a photo studio, the best way to proceed is as follows:

Split the pictures in different groups (chunks) according to the rotation that has been done. Align the different sets of pictures and calculate the (dense) point cloud. If the alignment is correct, the resulted point cloud needs to be cleaned. Now the different sets can be aligned and merged with each other using the information of the cleaned point cloud. In Agisoft Metashape for example, this is called "mask from model". If by any chance this does not work or the alignment of the groups is not as it should be, the masks based upon the point cloud can used to recalculate the first alignment of every subset of pictures.

\section{Processing}

A good detailed workflow on processing the point cloud and cleaning it can be found on https:// dinosaurpalaeo.wordpress.com/2015/10/11/photogrammetry-tutorial-11-how-to-handle-a-project-inagisoft-photoscan/, written by $\mathrm{H}$. Mallison.

If the alignment is correct, but the point cloud needs to be cleaned or other extensive processing is necessary before a dense point cloud or a mesh can be calculated, it is best to duplicate the alignment file so that one can always reprocess the original alignment with other parameters if the previous processing failed to give a good result.

Once the mesh can be calculated, choose the face count according to what is aimed to do (if it's for web visualisation a low polygon count is chosen; if it's for 3D printing it will depend on the resolution of the $3 \mathrm{D}$ printer; if the aim is to obtain the highest accuracy, the input can be a custom count to create the maximum possible). Normally it is always possible to perform a decimation of the mesh afterwards. But beware that sometimes the models can have a lot of polygons, sometimes too many for the machine to handle the 3D model fluently.

Often there is also the option to extrapolate the model or not. For instance, if certain parts of the object are not visible on multiple pictures no points are created and the algorithm will create something, assuming the shape. It is important to consider whether a hole is preferred or a closed, clean looking, but less correct mesh.

One of the final steps is to calculate the texture. It is our experience that sometimes the texture will have less contrast than the original pictures. A way to solve this is to export the created texture file and change the contrast settings in photo-processing software.

One feature that is important when producing SfM 3D models is the ability to scale the produced model. Sometimes only professional versions allow this step. It can be done in free 3D-processing software, but it is a lot easier if it can be done within the SfM software based on the scale that is pictured together with the object.

If the rotations do not align in one chunk, try aligning them in different chunks and align them two by two.

Avoid running other tasks on the computer while aligning the pictures or aligning two models at the same time on one workstation, which often causes failing alignments.

If the computer configuration allows it, activate GPU. This will speed up the processing.

If the 3D model that is calculated has small details like legs of an insect or small hair, the pictures can be masked and the "visibility consistent mesh generation method" can be used. This will improve the model for thin parts like hairs (only if masked) but is more time consuming. 


\subsection{Workflow Structured Light Scanner (HDI and MechScan)@RBINS}

It is not necessary to recalibrate the scanner every scanning day, but always recalibrate when the field of view is changed, the scanner is transported, or whenever there are big changes in temperature. Calibration is done by taking recordings of a vertically placed calibration board at different positions (front, middle and back) where the object will be located. Usually this will be on a turntable. For each position, move the scanner using the tripod head and record different pictures each time.

When the calibration is done, the specimen is placed on the turntable or at the previous middle position to check if the black cross (software view) matches with the red cross (camera view). Now calibrate the turntable by placing the calibration board horizontally.

Once both the scanner and the turntable are calibrated the object can be scanned. Usually 8 scans for each rotation are recorded. The scans are combined after a short cleaning process to delete the background or parts that are not accurately presented.

The next rotation is started after the object is placed in another position or if the scanner is placed in another viewpoint to the object. The field of view, i.e., the relative position of the cameras to the scanner need to stay the same.

For each rotation clean and combine the scans and subsequently combine the rotations with each other till the 3D model is complete. Now it can be exported.

Always scan in a room with constant lighting, preferably dark: a change in luminosity can create unwanted artefacts. Environmental conditions (light, temperature, etc.) should be the same as during calibration.

Avoid having too much scans. More scans than necessary only adds noise and renders the project heavier.

For objects with high colour contrast the "HDR" mode can be activated (select in the preferences). This mode is more time consuming, however.

The "Flexible" mode can be selected to scan in a less organized, more manual way.

The "Easy Scan" mode analyses the correct exposure for the user, which can be helpful from time to time.

\subsection{Workflow Laser Scanning@RBINS}

The NextEngine is a self-calibrated scanner, therefore no calibration is required.

Open StudioScan/StudioScanHD and press on the "Scan" button. This does not initiate a scan but opens the scan window in order to parameter the scan.

Positioning: $360^{\circ}$

Division: number of scans in one rotation, choose according to the complexity of the object, but usually between 8 and 12 should be enough.

Precision: if a small object is scanned or a higher precision is needed, choose "macro", otherwise choose "wide"

If macro is chosen, the object should be placed at $16.5 \mathrm{~cm}$ (! distance to the surface of the object to be scanned, not to the centre of the object). If the wide option is chosen, the object should be placed at $43 \mathrm{~cm}$. 
On the preview window, check that the object is well centred (use the rotation arrows on top to check that it is well centred and will stay in the view when rotated). A selection window can be drawn on the preview if only a small part of the view needs to be scanned (faster scan and less cleaning required afterwards).

Now it is possible to start scanning.

\section{Processing the scans}

The different parts of the scans are aligned together by manually placing common landmarks (a minimum of three) on the each scan, allowing the software to generate a precise alignment.

Cleaning can be done before or after alignment. Remove any unwanted parts. If there is a lot of overlapping data it can be interesting to remove side data of the scans where the colour is not good (blueish) in order to achieve a better texture.

Finally, fuse the scans together and export the finalized model.

\subsection{Workflow Infrared Depth Sensors@RMCA and RBINS}

\section{Workflow with Gotcha}

Connect the depth sensor and launch the Gotcha software. According to Mephisto the PrimeSense devices are factory calibrated but for more accurate results it is better to start with a calibration. This is done by scanning a checker pattern that is displayed on a screen. Around 15 pictures are necessary to calibrate the scanner.

When scanning, the normal mode is preferred. It is important to slowly move the scanner in a fluid motion at the correct distance from the object. If more points behind the object are recorded, the scanner is too close; if no points or objects in front of the specimen are recorded, the scanner is too far.

Abrupt movement will make sure the scanner is lost and scanning is stopped. This can also help if the computer and the object are far away from each other to end a scanning motion. Scan the object from different sides and combine the different scans by selecting common points on the scans. It helps sometimes to stick landmarks on or around the object to help this process. Finally, after cleaning and merging the model can be processed.

The technique is sensitive to differences in light. It is recommended to scan in controlled light environments, by placing tents around the object or using studio lights to evenly expose the object.

After merging the scans, it is possible to export the point cloud and mesh it in third-party software like GOM inspect, if the meshing of the original software is not good enough.

To make it easier to merge together all of the different scanned fragments, it really helps to rename each subscan a logical name which makes it clear which part is scanned.

\section{Workflow with ReconstructMe}

Make sure the SDK of the used sensor is installed.

Go to device tab and select the depth sensor that is used.

Go to volume tab and adjust the volume that needs to be scanned and the offset of the sensor.

In the handling volume the scan time can be adjusted. 
When all the parameters are set, click on start and start scanning moving the sensor slowly around the object.

Click on finish when a scanning motion is done.

Unlike Gotcha, the free version of ReconstructMe does not allow the alignment of different scans. In order to do that it is necessary to use a third-party software like MeshLab, Geomagic or others.

\subsection{D Formats and Post-Processing}

The information presented here on 3D formats and post-processing is not exhaustive. Several operations are briefly presented in order to give an introduction to different post-processing software. Most software mentioned here are freely available. For the moment there has not been an agreement between institutes at any level concerning which 3D formats should be used when distributing 3D data. This is something which should be decided within the framework of the ongoing digitisation initiatives as more and more institutes start digitising their collections in 3D.

\subsubsection{D Formats}

Many 3D formats are available. Some are proprietary formats that can only be opened by a specific software while others are interchangeable formats that can be opened in several software.

The formats mostly used at RBINS and RMCA are Wavefront OBJ (.OBJ), Stanford Triangle Format or Polygon File Format (.PLY), Collada (.DAE) and Standard Triangle Language (.STL), all of which are interchangeable formats that can be used with many types of 3D software.

The formats .OBJ and .DAE are mostly used for objects with texture files. To upload textured models on Sketchfab .OBJ is generally chosen. To import objects in Unity (Game Engine), .DAE gives better results than .OBJ. In general, avoid spaces or special characters in the name of the file; especially with .OBJ (if there is a space in the title it wo not be able to find the texture file anymore). Also, for formats with a texture file, make sure they are always saved in the same folder.

.PLY is a format used to record vertices colour information, while.STL is normally used when no colour information is recorded and is commonly used in $3 \mathrm{D}$ printing.

\subsubsection{Optimising/decimation}

Optimizing or decimating a model is a step used to simplify the model in order to have a smaller file (allowing for easier use). It is sometimes difficult to choose the right amount of decimations, because if the model is decimated too much, detail will be lost.

Many options are available to perform decimation. Often the 3D scanning software used, e.g., Agisoft or Flexscan, will allow direct decimating of the model in the software. But third-party software can also be employed, e.g., Meshlab (free and open source), GOM Inspect (freeware), Blender (free and open source), Instant Meshes (free and open source), Geomagic (paid), Balancer Pro (paid), and many more.

\section{Decimation workflow in Meshlab}

Meshlab offers different tools to decimate meshes. Mostly the "Quadric Edge Collapse Decimation" is used (Filters $>$ Remeshing, simplification and construction > Simplification: Quadric Edge Collapse Decimation). It offers the possibility to decimate textured mesh or mesh without a texture. It is important because when the mesh comes with a texture file, as is the case for .OBJ, .DAE and some .PLY, the texture coordinates of the model need to be preserved. 
A window opens offering different options:

- Target number of faces (set the number wished, by default it proposes $50 \%$ reduction)

- Quality threshold (default value of 0.3)

Activate "Preserve boundary of the mesh", "Optimal position of simplified vertices", "Preserve Normal" and "Planar Simplification". These parameters will ensure that the most accurate mesh is preserved while simplifying it to a maximum degree.

- Apply

- Export mesh (File > Export Mesh As) to save an optimized 3D model

\subsubsection{Cleaning}

As for optimizing, many 3D digitisation software will enable cleaning within the software and many options of third-party software are available. If the 3D model has a texture, it is best to clean it in Meshlab. If the 3D model does not have a texture, generally GOM Inspect or MeshMixer is used.

\section{Workflow in GOM Inspect}

Select and delete: GOM Inspect has a lot of selection tools. They can be selected from the toolbar at the bottom of the screen. First big elements can be removed using the "Select/Deselect Through Surface" tool.

- Select the part that needs to be removed using the left mouse button, then once everything is selected, right-click and choose selection + symbol.

- Press Ctrl+Delete to remove the selected parts (warning: do not just press delete or the entire model will be deleted).

- When all the large parts are removed the small isolated parts can be removed using "Select Patch".

- Left-click on the main model and the invert selection and Ctrl+Delete to remove the selection.

Once all the undesired parts are removed there are still a few operations that are possible to do to clean the mesh.

Normal orientation: if needed, select the part of the mesh where the normals are inverted and invert selected normals (Operations $>$ Mesh $>$ Invert Selected Normals). Or if only a few faces around the mesh are inverted, choose to automatically invert normals (Operations $>$ Mesh $>$ Automatically Orient Selected Normals).

If the mesh has holes, these can be closed using the "Close holes interactively" tool (Operations > Mesh $>$ Close holes $>$ Interactively): Ctrl+click on the edge of the hole to close and choose the filling results that give the best results (usually "Normal" or "Smooth") and leave "Delete neighborhood" on 0 or 1.

Finally, "Eliminate mesh errors" (Operations $>$ Mesh $>$ Other $>$ Eliminate Mesh Errors...) can be used but be careful as in some cases it will generate new artefacts.

\subsubsection{Meshing}

Some 3D scanning software only generates point clouds or sometimes the mesh produced in the native software is not the desired one, in which case third-party software such as Geomagic, MeshLab or CloudCompare (free and open source) can be used to mesh the point cloud. 


\section{Workflow in CloudCompare}

Import the point cloud in CloudCompare by dragging the file into the screen or using File $>$ Open $>$ select the point cloud. Apply the default parameters of import. Make sure the point cloud was saved with normals otherwise it cannot be meshed.

Select the cloud in the DB Tree.

Now open the "Poison" tool ("Plugins" > "Poison Surface Reconstruction"). A window with different options opens. The first parameter to play with is the "Octree depth". The higher the octree, the more precise the mesh will be but also the more time (and memory) consuming it will be. The use of 8 octree minimum is advised.

"Apply" and a new Mesh element has appeared in the DB Tree. If the result is not good enough repeat the process augmenting gradually the octree.

\subsubsection{Image processing}

Texture processing can be applied in order to improve contrast, colour and sharpness of a 3D scan texture. Almost any image processing software can be use. At RBINS and RMCA DxO Optics Pro is used. When processing an image on a non-calibrated screen, it might look great, but it can look differently on a calibrated screen or when printed.

\section{Texture processing with DxO OpticsPro (version 11)}

- Open the texture in DxO (Organize tab, Folders) and make sure no automatic preset is activated, otherwise choose the preset "no correction".

- Activate contrast and add some micro-contrast.

- The exposure compensation can be adjusted and RGB white balance for brightness and colour correction.

- A pre-set can be stored in case the same settings are often used ("Preset editor" > "New pre-set from current settings").

\section{Contributions}

This handbook wasn't possible without the contributions of the following people:

Bernhard Ströbel ${ }^{1}$, Michael Heethoff ${ }^{2}$, Susan Stephan ${ }^{3}$, Bernhard Schurian ${ }^{3}$, Didier VandenSpiegel ${ }^{4}$ and Patrick Semal ${ }^{5}$.

\section{Affiliations}

${ }^{1}$ Department of Mathematics and Natural Sciences,University of Applied Sciences Darmstadt, Schöfferstr. 3, D-64295 Darmstadt, Germany.

${ }^{2}$ Ecological Networks, Technische Universität Darmstadt, Schnittspahnstr. 3, D-64287 Darmstadt Germany.

${ }^{3}$ Museum für Naturkunde, Leibniz-Institut für Evolutions- und Biodiversitätsforschung, Invalidenstraße 43, D-10115 Berlin, Germany.

${ }^{4}$ Royal Museum for Central Africa, Biological Collections and Data Management, Leuvensesteenweg 13, B-3080 Tervuren, Belgium.

${ }^{5}$ Royal Belgian Institute of Natural Sciences, Scientific Heritage Service, Vautierstraat 29, B-1000 Brussels, Belgium. 
They contributed in the following way to the handbook:

Bernhard Ströbel and Michael Heethoff give their critical view on the entire paper and added content to the chapters 2.1, 2.3 and 3.1. Susan Stephan and Bernhard Schurian provided content for the chapter 2.3 and 9.3. Both Patrick Semal and Didier VandenSpiegel provided research ideas and feedback.

\section{Acknowledgements}

The creation of this handbook was funded by the EU FP7 program SYNTHESYS3 further supported by CETAF (Consortium of European Taxonomic Facilities), AISBL (Association Internationale Sans But Lucratif) organization (Ref no. BE825.688.249) and BELSPO (Belgian Science Policy). The authors would like to thank all the SYNTHESYS participants for their contribution to this handbook and especially Alexander Kroupa, Vincent S. Smith (Natural History Museum, UK) and Elspeth M. Haston (The Royal Botanic Garden Edinburgh, UK) for their support. The authors would also like to thank reviewers for providing comments and suggestions that improved the manuscript.

Manuscript received: 3 July 2019

Manuscript accepted: 16 July 2019

Published on: 6 April 2020

Topic editor: Koen Martens

Desk editor: Jeroen Venderickx

Printed versions of all papers are also deposited in the libraries of the institutes that are members of the EJT consortium: Muséum national d'Histoire naturelle, Paris, France; Meise Botanic Garden, Belgium; Royal Museum for Central Africa, Tervuren, Belgium; Royal Belgian Institute of Natural Sciences, Brussels, Belgium; Natural History Museum of Denmark, Copenhagen, Denmark; Naturalis Biodiversity Center, Leiden, the Netherlands; Museo Nacional de Ciencias Naturales-CSIC, Madrid, Spain; Real Jardín Botánico de Madrid CSIC, Spain; Zoological Research Museum Alexander Koenig, Bonn, Germany; National Museum, Prague, Czech Republic. 